\title{
Switching to nonhyperbolic cycles from codimension two bifurcations of equilibria of delay differential equations
}

\author{
M.M. Bosschaert* $\quad$ S.G. Janssens ${ }^{\dagger} \quad$ Yu.A. Kuznetsov ${ }^{\ddagger}$
}

March 19, 2019

\begin{abstract}
In this paper we perform the parameter-dependent center manifold reduction near the generalized Hopf (Bautin), fold-Hopf, Hopf-Hopf and transcritical-Hopf bifurcations in delay differential equations (DDEs). This allows us to initialize the continuation of codimension one equilibria and cycle bifurcations emanating from these codimension two bifurcation points. The normal form coefficients are derived in the functional analytic perturbation framework for dual semigroups (sun-star calculus) using a normalization technique based on the Fredholm alternative. The obtained expressions give explicit formulas which have been implemented in the freely available numerical software package DDE-BifTool. While our theoretical results are proven to apply more generally, the software implementation and examples focus on DDEs with finitely many discrete delays. Together with the continuation capabilities of DDE-BifTool, this provides a powerful tool to study the dynamics near equilibria of such DDEs. The effectiveness is demonstrated on various models.
\end{abstract}

Keywords: Generalized Hopf (Bautin) bifurcation, fold-Hopf bifurcation, Hopf-Hopf bifurcation, transcritical-Hopf bifurcation, codimension two bifurcation, normal forms, nonhyperbolic cycles, branch switching, delay differential equations, Center Manifold Theorem, adjoint operator semigroups, sun-star calculus, DDE-BifTool

2010 MSC: 34K18, 34K19, 34K60, 37L10, 37M20

\section{Introduction}

Great interest has recently been shown in the analysis of degenerate Hopf bifurcations in delay differential equations (DDEs), see e.g. $[1,17,18,25,35,36,39,40,48,50,51,54,57,61,64,65,66]$. In the simplest case, often encountered in applications, such DDEs have the form

$$
\dot{x}(t)=f\left(x(t), x\left(t-\tau_{1}\right), \ldots, x\left(t-\tau_{m}\right), \alpha\right), \quad t \geq 0,
$$

where $x(t) \in \mathbb{R}^{n}, \alpha \in \mathbb{R}^{p}, f: \mathbb{R}^{n \times(m+1)} \times \mathbb{R}^{p} \rightarrow \mathbb{R}^{n}$ is a smooth mapping and the delays $0<\tau_{1}<$ $\cdots<\tau_{m}$ are constant. They are known as discrete DDEs or DDEs of point type.

\footnotetext{
*Department of Mathematics, Hasselt University, Diepenbeek Campus, Agoralaan Gebouw D, 3590 Diepenbeek, Belgium (maikel.bosschaert@uhasselt.be).

${ }^{\dagger}$ Department of Mathematics, Utrecht University, Budapestlaan 6, 3508 TA Utrecht, The Netherlands (s.g.janssens@uu.nl, sj@dydx.nl).

$\ddagger$ Department of Mathematics, Utrecht University, Budapestlaan 6, 3508 TA Utrecht, The Netherlands and Department of Applied Mathematics, University of Twente, Zilverling Building, 7500AE Enschede, The Netherlands (I.A.Kouznetsov@uu.nl).
} 
Using the framework of perturbation theory for dual semigroups developed in $[4,5,6,7]$ the existence of a finite dimensional smooth center manifold for DDEs can be rigorously established [14]. As a consequence the normalization method for local bifurcations of ODEs developed in [42] can be lifted [37] rather easily to the infinite dimensional setting of DDEs. One of the advantages of this normalization technique is that the center manifold reduction and the calculation of the normal form coefficients are performed simultaneously by solving the so-called homological equation. The method gives explicit expressions for the coefficients rather than a procedure as developed in [22,23]. The critical normal form coefficients for all five generic codimension two bifurcations of equilibria of DDEs have been derived [37] and partially implemented [60] into the fully GNU Octave compatible MATLAB package DDE-BifTool $[21,56]$.

In this paper we will perform the parameter-dependent center manifold reduction and normalization for three codimension two Hopf cases: the generalized Hopf, fold-Hopf and Hopf-Hopf bifurcations. This will allow us to initialize the continuation of codimension one bifurcation curves of nonhyperbolic equilibria and cycles emanating from the codimension two points. These are the only codimension two bifurcation points of equilibria in generic DDEs where codimension one bifurcation curves of nonhyperbolic cycles could originate. We also treat the more special transcritical-Hopf bifurcation which is frequently found in applications.

The center manifold theorem for parameter-dependent DDEs as presented in [14] assumes explicitly that the steady state exists for all nearby parameter values. However, for a generic fold-Hopf bifurcation this assumption is not satisfied. An attempt to deal with this complication has been made in [30], where it is discussed how to reduce a parameter-dependent DDE to a DDE without parameters by appending the trivial equation $\dot{\alpha}=0$. However, the reduction in [30] is based on the formal adjoint approach [32] and applies specifically to DDEs, while at times it lacks consistency. Therefore we demonstrate in this paper how the reduction to the parameter-independent case can be done in the sun-star framework, enabling a rigorous approach to the existence of parameter-dependent center manifolds for a class of evolution equations that includes DDEs. This allows us to treat bifurcations of equilibria with zero eigenvalues in generic DDEs while at the same time achieving applicability of our results to other classes of delay equations.

This paper is organized as follows. In Section 2 we offer a concise review of perturbation theory for dual semigroups (also called sun-star calculus), both on an abstract level as well as in application to the analysis of classical DDEs as dynamical systems. We also recall from [37] various results that are needed for the normalization.

In Section 3 we show how the theory from the previous section also applies to parameter-dependent classical DDEs by converting them into a parameter-independent system on a product state space. We again present the material in two stages: Results are first established at a more abstract semigroup level and next applied to classical DDEs depending on parameters. In particular, we define the parameterdependent local center manifold and give an explicit ODE for solutions that are confined to it.

In Section 4 we describe the general technique used to derive expressions for the normal form coefficients on the parameter-dependent center manifold in the infinite dimensional setting of classical DDEs.

Before we apply this technique to the previously mentioned codimension two bifurcations, we summarize in Section 5 relevant smooth normal forms and we list asymptotics for the codimension one cycle bifurcation curves emanating from the codimension two points as well as for the corresponding nonhyperbolic equilibria.

In Section 6 the method is then applied to the generalized Hopf (Bautin), fold-Hopf, Hopf-Hopf and transcritical-Hopf bifurcations in classical DDEs. We provide explicit expressions for all normal form coefficients necessary for the predictors of codimension one bifurcation curves. While most of the critical normal form coefficients for these bifurcations were obtained in [37], we briefly re-derive them to ensure readability.

In Section 7 we provide explicit computational formulas for the evaluation of the linear and multilinear forms used in the normal form coefficients and predictors for the simplest subclass consisting of 
DDEs (1) with finite many discrete delays. These formulas are actually implemented in DDE-BifTool.

In Section 8 we employ our implementation in DDE-BifTool to illustrate the accuracy of the codimension one bifurcation curve predictors through various example models displaying all aforementioned degenerate Hopf cases. A complete step-by-step walk-through of the examples, including all code to reproduce the obtained results, is provided in the Supplement.

\section{Dual perturbation theory and classical DDEs}

We begin by presenting those general elements of perturbation theory for dual semigroups that are useful for the study of classical DDEs as dynamical systems. Throughout we assume sun-reflexivity - a term that will be introduced in Section 2.1. From Section 2.4 onward, we then explain how the general results apply to classical DDEs. The standard reference for this entire section is [14], while for the underlying theory of semigroups of linear operators we recommend [19,20].

\subsection{Duality structure and linear perturbation}

The starting point is a $\mathcal{C}_{0}$-semigroup $T_{0}$ on a real or complex Banach space $X$. Let $A_{0}$ with domain $\mathcal{D}\left(A_{0}\right)$ be the infinitesimal generator (or: generator, for short) of $T_{0}$. We denote by $X^{\star}$ the topological dual space of $X$ and we use the prefix notation for the pairing between $x^{\star} \in X^{\star}$ and $x \in X$,

$$
\left\langle x^{\star}, x\right\rangle:=x^{\star}(x) .
$$

If $X$ is not reflexive then the adjoint semigroup $T_{0}^{\star}$ is in general only weak ${ }^{\star}$ continuous on $X^{\star}$ and $A_{0}^{\star}$ generates $T_{0}^{\star}$ only in the weak ${ }^{\star}$ sense. The maximal subspace of strong continuity

$$
X^{\odot}:=\left\{x^{\star} \in X^{\star}: t \mapsto T_{0}^{\star}(t) x^{\star} \text { is norm-continuous on } \mathbb{R}_{+}\right\}
$$

is invariant under $T_{0}^{\star}$ and we have the characterization

$$
X^{\odot}=\overline{\mathcal{D}\left(A_{0}^{\star}\right)}
$$

where the bar denotes the norm closure in $X^{\star}$. By construction the restriction of $T_{0}^{\star}$ to $X^{\odot}$ is a $\mathcal{C}_{0}$-semigroup that we denote by $T_{0}^{\odot}$. Its generator $A_{0}^{\odot}$ is the part of $A_{0}^{\star}$ in $X^{\odot}$,

$$
\mathcal{D}\left(A_{0}^{\odot}\right)=\left\{x^{\odot} \in \mathcal{D}\left(A_{0}^{\star}\right): A_{0}^{\star} x^{\odot} \in X^{\odot}\right\}, \quad A_{0}^{\odot} x^{\odot}=A_{0}^{\star} x^{\odot} .
$$

At this stage we again have a $\mathcal{C}_{0}$-semigroup $T_{0}^{\odot}$ with generator $A_{0}^{\odot}$ on a Banach space $X^{\odot}$ so we can iterate the above construction. On the dual space $X^{\odot \star}$ we obtain the adjoint semigroup $T_{0}^{\odot \star}$ with weak $^{\star}$ generator $A_{0}^{\odot \star}$. By restriction to the maximal subspace of strong continuity $X^{\odot \odot}=\overline{\mathcal{D}\left(A_{0}^{\odot \star}\right)}$ we end up with the $\mathcal{C}_{0}$-semigroup $T_{0}^{\odot \odot}$. Its generator $A_{0}^{\odot \odot}$ is the part of $A_{0}^{\odot \star}$ in $X \odot \odot$.

The canonical injection $j: X \rightarrow X^{\odot \star}$ defined by

$$
\left\langle j x, x^{\odot}\right\rangle:=\left\langle x^{\odot}, x\right\rangle
$$

maps $X$ into $X \odot \odot$. If $j$ maps $X$ onto $X^{\odot \odot}$ then $X$ is called $\odot$-reflexive (pronounce: sun-reflexive) with respect to $T_{0}$. One may define an equivalent norm on $X$ with respect to which $j$ becomes an isometry, but this need not be assumed. However, sun-reflexivity of $X$ with respect to $T_{0}$ will be assumed throughout.

With the abstract duality structure in place, we next turn our attention to perturbation. Let $L: X \rightarrow X^{\odot \star}$ be a bounded linear operator. Then there exists a unique $\mathcal{C}_{0}$-semigroup $T$ on $X$ that satisfies the linear integral equation

$$
T(t) x=T_{0}(t) x+j^{-1} \int_{0}^{t} T_{0}^{\odot \star}(t-\tau) L T(\tau) x d \tau, \quad t \geq 0, x \in X
$$


where the weak ${ }^{\star}$ Riemann integral takes values in $X^{\odot \odot}$ and the running assumption of sun-reflexivity justifies the application of $j^{-1}$. By using (3) to express the difference $T-T_{0}$ of the perturbed and the unperturbed semigroups, one proves that the maximal subspaces of strong continuity $X^{\odot}$ and $X^{\odot \odot}$ are the same for $T$ and $T_{0}$, so there is no need to distinguish them with a subscript. In particular, $X$ is sun-reflexive also with respect to $T$. On $X^{\odot \star}$ the perturbation $L$ appears additively in the action of $A^{\odot \star}$,

$$
\mathcal{D}\left(A^{\odot \star}\right)=\mathcal{D}\left(A_{0}^{\odot \star}\right), \quad A^{\odot \star}=A_{0}^{\odot \star}+L j^{-1} .
$$

We recover the generator $A$ of $T$ by considering the part of $A^{\odot \star}$ in $X^{\odot \odot}$. As a consequence $L$ moves into the domain and we find

$$
\mathcal{D}(A)=\left\{x \in X: j x \in \mathcal{D}\left(A_{0}^{\odot \star}\right) \text { and } A_{0}^{\odot \star} j x+L x \in X^{\odot \odot}\right\}, \quad A x=j^{-1}\left(A_{0}^{\odot \star} j x+L x\right) .
$$

For proofs of the statements so far, see [14, Appendix II.3 and Chapter III].

\subsection{Nonlinear perturbation and linearization}

The $\mathcal{C}_{0}$-semigroup $T$ arose as a linear perturbation of the original $\mathcal{C}_{0}$-semigroup $T_{0}$, so the next step is to introduce a nonlinear perturbation of $T$ itself. In keeping with the tradition for nonlinear problems [14, Sections VII.1 and VIII.1] we only regard the case that $X$ is a real Banach space, also see Remark 1 below. Let $R: X \rightarrow X^{\odot \star}$ be a $C^{k}$-operator for some $k \geq 1$ such that

$$
R(0)=0, \quad D R(0)=0,
$$

and consider the nonlinear integral equation

$$
u(t)=T(t) x+j^{-1} \int_{0}^{t} T^{\odot \star}(t-\tau) R(u(\tau)) d \tau, \quad t \geq 0, x \in X .
$$

Due to the nonlinearity, for a given initial condition $x \in X$ one can at most guarantee existence of a maximal solution $u_{x}: I_{x} \rightarrow X$ of (5) on a forward time interval $I_{x}:=\left[0, t_{x}\right)$ for some $0<t_{x} \leq \infty[14$, Chapter VII]. The family of all such maximal solutions defines a nonlinear semiflow $\Sigma: \mathcal{D}(\Sigma) \rightarrow X$,

$$
\mathcal{D}(\Sigma):=\left\{(t, x) \in[0, \infty) \times X: t \in I_{x}\right\}, \quad \Sigma(t, x):=u_{x}(t),
$$

that may in addition depend on parameters [14, Defs. VII.2.1 and VII.2.9]. (For reasons discussed in Section 3, we will treat parameter dependence differently and separately. Until then, the reader can consider all parameters to be held fixed and absent in the notation.) The domain of $\Sigma$ is open in $[0, \infty) \times X$ and $0 \in X$ is a stationary point of $\Sigma$,

$$
I_{0}=[0, \infty), \quad \Sigma(t, 0)=0, \quad \forall t \geq 0 .
$$

The semiflow $\Sigma$ is (in fact, uniformly) differentiable with respect to the state at $(t, 0) \in \mathcal{D}(\Sigma)$, with the partial derivative

$$
D_{2} \Sigma(t, 0)=T(t), \quad \forall t \geq 0,
$$

where $T$ is the $\mathcal{C}_{0}$-semigroup that satisfies (3).

Remark 1. For nonlinear problems it is customary to work on a real Banach space $X$. The reason is that these problems often come from concrete equations with nonlinear right-hand sides for which it is unclear if and how they can be extended to complex arguments. Consequently, if we want to analyze the linearization of $\Sigma$ at $0 \in X$ using spectral theory, then it becomes necessary to complexify $X$ and the linear operators acting on $X$ [14, Section III.7 and last part of Section IV.2], [52, Section 1.3]. In particular, by the spectrum of $A$ we mean the spectrum of its complexification on the complexified Banach space. 


\subsection{Critical local center manifolds}

As in Section 2.2 we continue to assume that $T_{0}$ is a $\mathcal{C}_{0}$-semigroup on a real Banach space $X$ that is sun-reflexive with respect to $T_{0}$. In addition we assume that $T_{0}$ is eventually compact and $L$ is a compact operator. This implies that the perturbed semigroup $T$ defined by (3) is eventually compact as well [11, Theorem 2.8].

When considering solutions that exist for all (positive and negative) time - such as periodic orbits - it is useful to write (5) in the translation invariant form

$$
u(t)=T(t-s) u(s)+j^{-1} \int_{s}^{t} T^{\odot \star}(t-\tau) R(u(\tau)) d \tau, \quad-\infty<s \leq t<\infty .
$$

A solution of (8) is a continuous function $u: I \rightarrow X$ on some nondegenerate - possibly unbounded interval $I \subseteq \mathbb{R}$ that satisfies (8) for all $s, t \in I$ with $s \leq t$. Naturally, $u$ is a solution of (8) if and only if

$$
t-s \in I_{u(s)}, \quad u(t)=\Sigma(t-s, u(s)), \quad \forall s, t \in I \text { with } s \leq t,
$$

where $\Sigma: \mathcal{D}(\Sigma) \rightarrow X$ is the nonlinear semiflow from (6). The interval $I$ is often left implicit.

The general center manifold theorems from [14, Chapter IX] for equations of the type (8) apply to the particular case where $T$ is an eventually compact $\mathcal{C}_{0}$-semigroup on a real, sun-reflexive Banach space. Let us therefore suppose that $0 \in X$ is a nonhyperbolic equilibrium of $\Sigma$, so the generator $A$ of $T$ possesses $1 \leq n_{0}<\infty$ purely imaginary eigenvalues, counting algebraic multiplicities - see Remark 1 . Let $X_{0} \subseteq X$ be the real center eigenspace corresponding to these eigenvalues. Then there exists a $C^{k}$-smooth $n_{0}$-dimensional local center manifold $\mathcal{W}_{\text {loc }}^{c}$ that is tangent to $X_{0}$ at the origin. Any solution $u: I \rightarrow X$ of (8) that lies on $\mathcal{W}_{\text {loc }}^{c}$ is differentiable on $I$ and satisfies

$$
j \dot{u}(t)=A^{\odot \star} j u(t)+R(u(t)), \quad \forall t \in I,
$$

where $A^{\odot \star}$ is the weak ${ }^{\star}$ generator of $T^{\odot \star}$. We note that $(9)$ is an identity in $X^{\odot \star}$.

\subsection{The special case of classical DDEs}

It will now be explained how the general results from Sections 2.1 to 2.3 apply to classical DDEs. We choose the nonreflexive Banach space $X:=C\left([-h, 0], \mathbb{R}^{n}\right)$ as the state space, introduce a $C^{k}$-smooth operator $F: X \rightarrow \mathbb{R}^{n}$, and consider an equation with a finite delay $0<h<\infty$ of the form

$$
\dot{x}(t)=F\left(x_{t}\right), \quad t \geq 0
$$

with an initial condition

$$
x_{0}=\varphi \in X .
$$

For each $t \geq 0$, the function $x_{t}:[-h, 0] \rightarrow \mathbb{R}^{n}$ defined by

$$
x_{t}(\theta):=x(t+\theta), \quad \forall \theta \in[-h, 0],
$$

is called the history of the unknown function $x$ at time $t$. Equations of the type (DDE) will be called classical DDEs. Note that (1) is quite literally a case in point. By a solution of the initial value problem (DDE)-(IC) we mean a continuous function $x:\left[-h, t_{+}\right) \rightarrow \mathbb{R}^{n}$ for some $0<t_{+} \leq \infty$ that is differentiable on $\left[0, t_{+}\right)$and satisfies (DDE) and (IC). When $t_{+}=\infty$ we call $x$ a global solution.

We want to study (DDE) near an equilibrium at the origin, so assume that $F(0)=0$ and split $F$ into its linear and nonlinear parts,

$$
F(\varphi)=\int_{0}^{h} d \zeta(\theta) \varphi(-\theta)+G(\varphi), \quad \varphi \in X
$$


Here $\zeta:[0, h] \rightarrow \mathbb{R}^{n \times n}$ is a matrix-valued function of bounded variation, normalized by the requirement that $\zeta(0)=0$ and $\zeta$ is right-continuous on the open interval $(0, h)$. The integral is of the RiemannStieltjes type, and $G: X \rightarrow \mathbb{R}^{n}$ is a $C^{k}$-smooth nonlinear operator. It is common to denote the linear part more succinctly as

$$
\langle\zeta, \varphi\rangle:=\int_{0}^{h} d \zeta(\theta) \varphi(-\theta)
$$

so that

$$
F(\varphi)=\langle\zeta, \varphi\rangle+G(\varphi), \quad \varphi \in X
$$

We first consider the case $G=0$, whence (DDE) reduces to the linear equation

$$
\dot{x}(t)=\left\langle\zeta, x_{t}\right\rangle, \quad t \geq 0
$$

In order to understand the relationship between (12) and (3) we begin by observing that the trivial DDE

$$
\dot{x}(t)=0, \quad t \geq 0,
$$

with initial condition (IC) has the obvious solution

$$
x^{\varphi}(t)= \begin{cases}\varphi(t), & t \in[-h, 0] \\ \varphi(0), & t>0\end{cases}
$$

Using this solution, we define the strongly continuous shift semigroup $T_{0}$ on $X$ by

$$
\left(T_{0}(t) \varphi\right)(\theta):=x^{\varphi}(t+\theta)= \begin{cases}\varphi(t+\theta), & t+\theta \in[-h, 0] \\ \varphi(0), & t+\theta>0\end{cases}
$$

We note that $T_{0}(h)$ is a compact operator, so $T_{0}$ is eventually compact. For this particular combination of $X$ and $T_{0}$ the abstract duality structure from Section 2.1 can be constructed systematically and explicitly [14, Section II.5]. We only summarize the few facts that will be used in the sequel.

Remark 2 (Notation). For $\mathbb{K} \in\{\mathbb{R}, \mathbb{C}\}$ let $\mathbb{K}^{n}$ be the linear space of column vectors and let $\mathbb{K}^{n \star}$ be the linear space of row vectors, both over $\mathbb{K}$. Elements of $\mathbb{K}^{n}$ are denoted by $q=\left(q_{1}, q_{2}, \ldots, q_{n}\right)$ - commas between the entries - while elements in $\mathbb{K}^{n \star}$ are denoted by $p=\left(\begin{array}{llll}p_{1} & p_{2} & \cdots & p_{n}\end{array}\right)$ - no commas between the entries. We sometimes use the pairing defined by the row-column matrix multiplication:

$$
p \cdot q:=p q=\sum_{j=1}^{n} p_{j} q_{j}, \quad p \in \mathbb{K}^{n \star}, q \in \mathbb{K}^{n} .
$$

Note that the standard Hermitian inner product between two vectors $p^{T}, q \in \mathbb{C}^{n}$ should be written as $\bar{p} \cdot q$ and not as $p \cdot q$.

On $X^{\odot}$ : The maximal domain of strong continuity of $T_{0}^{\star}$ has the representation

$$
X^{\odot}=\mathbb{R}^{n \star} \times L^{1}\left([0, h], \mathbb{R}^{n \star}\right),
$$

and the duality pairing between $\varphi^{\odot}=(c, g) \in X^{\odot}$ and $\varphi \in X$ is

$$
\left\langle\varphi^{\odot}, \varphi\right\rangle=c \varphi(0)+\int_{0}^{h} g(\theta) \varphi(-\theta) d \theta .
$$


On $X^{\odot \star}$ : Switching to the dual space of (15) yields the representation

$$
X^{\odot \star}=\mathbb{R}^{n} \times L^{\infty}\left([-h, 0], \mathbb{R}^{n}\right),
$$

and the duality pairing between $\varphi^{\odot \star}=(a, \psi) \in X^{\odot \star}$ and $\varphi^{\odot}=(c, g) \in X^{\odot}$ is

$$
\left\langle\varphi^{\odot \star}, \varphi^{\odot}\right\rangle=c a+\int_{0}^{h} g(\theta) \psi(-\theta) d \theta
$$

The canonical injection (2) sends $\varphi \in X$ to $j \varphi=(\varphi(0), \varphi)$, mapping $X$ onto $X \odot \odot$. Therefore $X$ is sun-reflexive with respect to the shift semigroup $T_{0}$.

Next, we specify the linear and nonlinear perturbations $L$ and $R$ in (3) and (5), respectively, and to relate these two abstract integral equations in $X$ to the linear and nonlinear initial value problems for (DDE). For $i=1, \ldots, n$ we denote $r_{i}^{\odot \star}:=\left(e_{i}, 0\right)$ where $e_{i}$ is the $i$ th standard basis vector of $\mathbb{R}^{n}$. It is conventional and convenient to introduce the shorthand

$$
w r^{\odot \star}:=\sum_{i=1}^{n} w_{i} r_{i}^{\odot \star}, \quad \forall w=\left(w_{1}, \ldots, w_{n}\right) \in \mathbb{R}^{n},
$$

and we note that $w r^{\odot \star}=(w, 0) \in X^{\odot \star}$. First we define the compact linear perturbation in (3) as

$$
L \varphi:=\langle\zeta, \varphi\rangle r^{\odot *}
$$

where the pairing in the right-hand side is given by (10). Now (12) with (IC) is equivalent to (3) with (18) in the following sense: If $T$ is the unique $\mathcal{C}_{0}$-semigroup on $X$ satisfying (3) with (18) then $x^{\varphi}:[-h, \infty) \rightarrow \mathbb{R}^{n}$ defined by

$$
x_{0}^{\varphi}:=\varphi, \quad x^{\varphi}(t):=(T(t) \varphi)(0), \quad \forall t \geq 0,
$$

is the unique global solution of (12) with (IC) and

$$
x_{t}^{\varphi}=T(t) \varphi, \quad \forall t \geq 0 .
$$

It remains to specify the nonlinear perturbation $R$ in (5) as

$$
R(\varphi):=G(\varphi) r^{\odot \star},
$$

where $G$ is the nonlinear operator appearing in the splitting (11). Let $\Sigma$ as in (6) be the nonlinear semiflow generated by the family of maximal solutions of (5) with (19). The equivalence between (DDE)-(IC) and (5) with (19) can be formulated as follows [14, Prop. VII.6.1]. The function $x^{\varphi}$ : $\left[-h, t_{\varphi}\right) \rightarrow \mathbb{R}^{n}$ defined by

$$
x_{0}^{\varphi}:=\varphi, \quad x^{\varphi}(t):=\Sigma(t, \varphi)(0), \quad \forall t \in I_{\varphi},
$$

is the maximal solution of (DDE)-(IC), in the sense that any other solution necessarily exists only on a subinterval $\left[-h, t_{+}\right)$for some $0<t_{+} \leq t_{\varphi}$ and coincides with $x^{\varphi}$ there. Moreover,

$$
x_{t}^{\varphi}=\Sigma(t, \varphi), \quad \forall t \in I_{\varphi} .
$$

It is the content of (7) that generation and linearization commute: Starting with (DDE), linearization of the semiflow $\Sigma$ at the stationary point $0 \in X$ yields precisely the eventually compact $\mathcal{C}_{0}$-semigroup $T$ corresponding to the linearized DDE (12). 


\subsection{Spectral computations for classical DDEs}

The eventual compactness of $T$ implies that the spectrum of its generator $A$ - see Remark 1 - consists entirely of isolated eigenvalues of finite algebraic multiplicity. It is clear from (18) that $L$ is not just compact, but actually of finite rank. This implies that all spectral information about $A$ is contained in a holomorphic characteristic matrix function $\Delta: \mathbb{C} \rightarrow \mathbb{C}^{n \times n}$ defined by

$$
\Delta(z):=z I-\hat{\zeta}(z) \quad \text { with } \quad \hat{\zeta}(z):=\int_{0}^{h} e^{-z \theta} d \zeta(\theta)
$$

where $\zeta$ is the real kernel from (10) [14, Sections IV.4 and IV.5]. In particular, the eigenvalues of $A$ are the roots of the characteristic equation

$$
\operatorname{det} \Delta(z)=0
$$

and the algebraic multiplicity of an eigenvalue equals its order as a root of (21).

We will be concerned exclusively with simple eigenvalues, for which the geometric and algebraic multiplicities are both equal to one. Let $\lambda \in \mathbb{C}$ be such a simple eigenvalue of $A$. There exist nonzero right and left null vectors $q \in \mathbb{C}^{n}$ and $p \in \mathbb{C}^{n \star}$ of $\Delta(\lambda)$,

$$
\Delta(\lambda) q=0, \quad p \Delta(\lambda)=0 .
$$

The second equation is of course equivalent to $p^{T}$ being a nonzero right null vector of $\Delta^{T}(\lambda)$. The one-dimensional eigenspaces of $A$ and $A^{\star}$ corresponding to $\lambda$ are spanned by eigenfunctions $\varphi$ and $\varphi^{\odot}$, respectively, with

$$
\varphi(\theta)=e^{\lambda \theta} q, \quad \theta \in[-h, 0],
$$

and

$$
\varphi^{\odot}=\left(p, \theta \mapsto p \int_{\theta}^{h} e^{\lambda(\theta-\tau)} d \zeta(\tau)\right), \quad \theta \in[0, h] .
$$

We note that we have implicitly used - and will use consistently - the complexifications of $X$ and of the representation (15) of $X^{\odot}$. For a simple eigenvalue $\lambda$,

$$
\left\langle\varphi^{\odot}, \varphi\right\rangle \neq 0,
$$

where the duality pairing is understood to be the complexification of (16). This nonequality implies that the eigenfunctions can be normalized to satisfy $\left\langle\varphi^{\odot}, \varphi\right\rangle=1$. In fact, from (16) and (22) one computes

$$
\left\langle\varphi^{\odot}, \varphi\right\rangle=p \Delta^{\prime}(\lambda) q
$$

so this normalization can be effectuated by scaling $p$ and $q$ such that $p \Delta^{\prime}(\lambda) q=1$. Finally, it is easily seen that if $\mu \neq \lambda$ is another simple eigenvalue of $A$ with eigenvector $\psi$ and adjoint eigenvector $\psi^{\odot}$, then

$$
\left\langle\varphi^{\odot}, \psi\right\rangle=0, \quad\left\langle\psi^{\odot}, \varphi\right\rangle=0 .
$$

\subsection{Solvability of linear operator equations}

When computing the normal form coefficients in Section 6 using (HOM), we will encounter linear operator equations of the form

$$
\left(z I-A^{\odot \star}\right)\left(v_{0}, v\right)=\left(w_{0}, w\right),
$$

where $z$ is a complex number, $\left(w_{0}, w\right) \in X^{\odot \star}$ is given and $\left(v_{0}, v\right) \in D\left(A^{\odot \star}\right)$ is the unknown. In general, both $z$ and the right-hand side will have a nontrivial imaginary part, so here and from here onward, it is necessary to regard systems of the form (26) as the complexification of the original operator equations. 
We will however not attach additional subscripts to the operator symbols, hoping that this omission will not cause confusion.

Since $\sigma(A)$ consists exclusively of point spectrum, there are two situations to consider depending on whether or not $z$ is an eigenvalue. If $z$ is not an eigenvalue of $A$ then $z$ belongs to the resolvent set $\rho(A)$ of $A$ and (26) admits a unique solution,

$$
\left(v_{0}, v\right)=\left(z I-A^{\odot \star}\right)^{-1}\left(w_{0}, w\right) .
$$

In order to actually find this solution, one needs a representation of the resolvent operator of $A^{\odot \star}$. The general result can be found in [14, Corollary IV.5.4], but here we only require a special case.

Lemma 3. Suppose that $z$ is not an eigenvalue of $A$, so (26) has a unique solution $\left(v_{0}, v\right)$. If the right-hand side is represented by

$$
\left(w_{0}, w\right)=\left(w_{0}, \theta \mapsto e^{z \theta} \Delta^{-1}(z) \eta\right),
$$

for some fixed vector $\eta \in \mathbb{C}^{n}$, then this solution has the representation

$$
v_{0}=v(0), \quad v(\theta)=\Delta^{-1}(z)\left(e^{z \theta} w_{0}+\left(\Delta^{\prime}(z)-I-\theta \Delta(z)\right) w(\theta)\right) .
$$

Proof. Write $\left(w_{0}, w\right)=\left(w_{0}, 0\right)+\left(0, \theta \mapsto e^{z \theta} \Delta^{-1}(z) \eta\right)$, use the linearity of $\left(z I-A^{\odot \star}\right)^{-1}$ and apply both cases of [37, Corollary 3.4].

On the other hand, suppose that $z=\lambda$ is an eigenvalue. Then (26) need not be consistent. In fact, a solution exists if and only if

$$
\left\langle\left(w_{0}, w\right), \varphi^{\odot}\right\rangle=0, \quad \forall \varphi^{\odot} \in \mathcal{N}\left(\lambda I-A^{\star}\right),
$$

see [37, Lemma 3.2]). This condition is often referred to as the Fredholm solvability condition. We note that the duality pairing in (FSC) may be evaluated in concrete cases using (22) and the complexification of (17). This will be done many times in Section 6 when we apply (FSC) to specific operator equations.

If $z=\lambda$ is an eigenvalue and (26) is consistent, then clearly its solutions are not unique. The bordered operator inverse

$$
\left(\lambda I-A^{\odot \star}\right)^{\mathrm{INV}}: \mathcal{R}\left(\lambda I-A^{\odot \star}\right) \rightarrow \mathcal{D}\left(A^{\odot \star}\right),
$$

is used to select a particular solution in a systematic and convenient way. For the case that $\lambda$ is a simple eigenvalue, it assigns the unique solution of the extended linear system

$$
\left(\lambda I-A^{\odot \star}\right)\left(v_{0}, v\right)=\left(w_{0}, w\right), \quad\left\langle\left(v_{0}, v\right), \varphi^{\odot}\right\rangle=0,
$$

to every $\left(w_{0}, w\right)$ for which $(26)$ is consistent. The following lemma gives an explicit representation for a special case [37, Proposition 3.6 and Corollary 3.7].

Lemma 4. Let $z=\lambda$ be a simple eigenvalue with eigenvector $\varphi$ and adjoint eigenvector $\varphi^{\odot}$ as in (22), normalized to $\left\langle\varphi^{\odot}, \varphi\right\rangle=1$. Suppose (26) is consistent for a given right-hand side of the form

$$
\left(w_{0}, w\right)=(\eta, 0)+\kappa(q, \varphi)
$$

where $\eta \in \mathbb{C}^{n}$ and $\kappa \in \mathbb{C}$. Then the unique solution $\left(v_{0}, v\right)$ of $(27)$ is given by

$$
v_{0}=\xi+\gamma q, \quad v(\theta)=e^{\lambda \theta}\left(v_{0}-\kappa \theta q\right),
$$

with $\xi=\Delta^{\mathrm{INV}}(\lambda)\left(\eta+\kappa \Delta^{\prime}(\lambda) q\right)$ and $\gamma=-p \Delta^{\prime}(\lambda) \xi+\frac{1}{2} \kappa p \Delta^{\prime \prime}(\lambda) q$. 
In Section 6 we will use the shorthand notation

$$
v=B_{\lambda}^{\mathrm{INV}}(\eta, \kappa)
$$

for the solution in Lemma 4. We observe that the expression for $\xi$ itself involves a bordered matrix inverse,

$$
\Delta^{\mathrm{INV}}(\lambda): \mathcal{R}(\Delta(\lambda)) \rightarrow \mathbb{C}^{n}
$$

which assigns the unique solution of the extended linear system

$$
\Delta(\lambda) x=y, \quad p \cdot x=0,
$$

to every $y \in \mathbb{C}^{n}$ for which the system $\Delta(\lambda) x=y$ is consistent - also see Remark 2 for the notation. In practice, $x=\Delta^{\operatorname{INV}}(\lambda) y$ can be obtained by solving the nonsingular bordered matrix system

$$
\left(\begin{array}{cc}
\Delta(\lambda) & q \\
p & 0
\end{array}\right)\left(\begin{array}{l}
x \\
s
\end{array}\right)=\left(\begin{array}{l}
y \\
0
\end{array}\right)
$$

for the unknown $(x, s) \in \mathbb{C}^{n+1}$ that necessarily satisfies $s=0$. The properties of (finite dimensional) bordered linear systems and their role in numerical bifurcation analysis are discussed more extensively in [41] and [28, Chapter 3].

\section{Parameter dependence and classical DDEs}

In Section 3.1 we motivate our approach by explaining why the standard literature result does not apply to the problem at hand. This is most easily done at the concrete level of classical DDEs. The structure of the remaining subsections parallels that of Section 2. Namely, we first solve the problem of parameter dependence at the more abstract level of dual perturbation theory. In the final Section 3.6 we then return to classical DDEs to see how the general results apply in this special case.

\subsection{Motivation}

We are concerned with the situation where the right-hand side of (DDE) depends explicitly on parameters. Specifically, we consider

$$
\dot{x}(t)=F\left(x_{t}, \alpha\right), \quad t \geq 0,
$$

where $F: X \times \mathbb{R}^{p} \rightarrow \mathbb{R}^{n}$ is $C^{k}$-smooth for some $k \geq 1$ with $F(0,0)=0$. We assume that at the critical parameter value $\alpha=0$ the linearization of (28) has $1 \leq n_{0}<\infty$ purely imaginary eigenvalues, counting multiplicities. The goal of Section 3 is to obtain a parameter-dependent family of local center manifolds for a class of evolution equations that includes (28).

In [14, Section IX.9.1] this problem is approached as follows. One augments (28) with a trivial equation for the constant parameter dynamics. This gives the system

$$
\left\{\begin{array}{l}
\dot{x}(t)=F\left(x_{t}, \mu(t)\right), \quad t \geq 0, \\
\dot{\mu}(t)=0,
\end{array}\right.
$$

on the state space $\boldsymbol{X}:=X \times \mathbb{R}^{p}$, with $X=C\left([-h, 0], \mathbb{R}^{n}\right)$ as before in Section 2.4. Then the right-hand side of the first equation of (29) is split as

$$
F(\varphi, \alpha)=D_{1} F(0,0) \varphi+\tilde{G}(\varphi, \alpha)
$$

which defines $\tilde{G}: \boldsymbol{X} \rightarrow \mathbb{R}^{n}$, cf. [14, (9.7) in Section IX.9.1]. The first term on the right of (30) acts only on the $X$-component of the state in $\boldsymbol{X}$, so the semigroup $\tilde{\boldsymbol{T}}$ on $\boldsymbol{X}$ obtained by perturbing the shift-semigroup $\boldsymbol{T}_{0}$ is diagonal. 
However, there is an obstruction. In order to satisfy the hypotheses of the parameter-independent center manifold theorem, $\tilde{G}$ must be a pure nonlinearity on $\boldsymbol{X}$, i.e.

$$
\tilde{G}(0,0)=0, \quad D_{1} \tilde{G}(0,0)=0, \quad D_{2} \tilde{G}(0,0)=0 .
$$

The first two of these conditions are clearly fulfilled, but in general there is no reason for the third condition to be met. It does hold when $\tilde{G}(0, \alpha)=0$ for all $\alpha \in \mathbb{R}^{p}$ in a neighborhood of zero, i.e. when the zero equilibrium of (28) persists under small parameter variations. For a generic fold-Hopf bifurcation - as well as for a generic Bogdanov-Takens bifurcation that we do not discuss here - there is no such persistence.

In this article, the above difficulty is resolved by considering instead of (30) the splitting

$$
F(\varphi, \alpha)=D_{1} F(0,0) \varphi+D_{2} F(0,0) \alpha+G(\varphi, \alpha) .
$$

Using this splitting, (29) is written as

$$
\left\{\begin{array}{l}
\dot{x}(t)=D_{1} F(0,0) x_{t}+D_{2} F(0,0) \mu(t)+G\left(x_{t}, \mu(t)\right), \quad t \geq 0 . \\
\dot{\mu}(t)=0,
\end{array}\right.
$$

Now both $D_{1} F(0,0)$ as well as $D_{2} F(0,0)$ appear in the perturbation of $\boldsymbol{T}_{0}$. As a consequence the perturbed semigroup $\boldsymbol{T}$ is no longer diagonal, but still simple enough for a complete analysis. Moreover,

$$
G(0,0)=0, \quad D_{1} G(0,0)=0, \quad D_{2} G(0,0)=0,
$$

so the parameter-independent center manifold theorem can be applied without having to assume equilibrium persistence. Of course $G=\tilde{G}$ and $\boldsymbol{T}=\tilde{\boldsymbol{T}}$ whenever $D_{2} F(0,0)=0$.

Remark 5. In a first attempt we regarded the augmented system (29) as a DDE on the state space $C\left([-h, 0], \mathbb{R}^{n+p}\right)$, also see [30], but we found this approach to be a bit unsatisfactory: The proofs in Sections 3.2 to 3.5 do not depend on the details of the class of equations under consideration, while those same details sometimes lead to notation that is more complicated than necessary.

\subsection{Duality structure and linear perturbation}

We work in the setting of Section 2.1. Namely, let $T_{0}$ be a $\mathcal{C}_{0}$-semigroup on a real or complex Banach space $X$ that is sun-reflexive with respect to $T_{0}$. We write $\mathbb{K} \in\{\mathbb{R}, \mathbb{C}\}$ for the underlying scalar field as in Remark 2. Define $\boldsymbol{T}_{0}$ on $\boldsymbol{X}$ by

$$
\boldsymbol{T}_{0}(t):=\operatorname{diag}\left(T_{0}(t), I_{p}\right) .
$$

The procedure of taking adjoints and restrictions (twice) then yields semigroups $\boldsymbol{T}_{0}^{\star}, \boldsymbol{T}_{0}^{\odot}, \boldsymbol{T}_{0}^{\odot \star}$ and $\boldsymbol{T}_{0}^{\odot \odot}$ on $\boldsymbol{X}^{\star} \simeq X^{\star} \times \mathbb{K}^{p}, \boldsymbol{X}^{\odot} \simeq X^{\odot} \times \mathbb{K}^{p}, \boldsymbol{X}^{\odot \star} \simeq X^{\odot \star} \times \mathbb{K}^{p}$ and $\boldsymbol{X}^{\odot \odot} \simeq X^{\odot \odot} \times \mathbb{K}^{p}$. (The symbol $\simeq$ indicates an identification via a natural isometric isomorphism.) It is straightforward to check that on $\boldsymbol{X}^{\odot \star}$ we have

$$
\boldsymbol{T}_{0}^{\odot \star}(t)=\operatorname{diag}\left(T_{0}^{\odot \star}(t), I_{p}\right)
$$

and that the canonical injection $\boldsymbol{j}: \boldsymbol{X} \rightarrow \boldsymbol{X}^{\odot \star}$ has the form

$$
\boldsymbol{j}=\operatorname{diag}\left(j, I_{p}\right)
$$

where $j: X \rightarrow X^{\odot \star}$ is the canonical injection from (2). In particular, $\boldsymbol{X}$ is sun-reflexive with respect to $\boldsymbol{T}_{0}$.

As in Section 2.1 we now introduce a bounded linear perturbation $\boldsymbol{L}: \boldsymbol{X} \rightarrow \boldsymbol{X}^{\odot \star}$ of $\boldsymbol{T}_{0}$. We let it be of the form

$$
\boldsymbol{L}=\left(\begin{array}{cc}
L & L_{p} \\
0 & 0
\end{array}\right)
$$

with $L: X \rightarrow X^{\odot \star}$ and $L_{p}: \mathbb{K}^{p} \rightarrow X^{\odot \star}$ bounded linear operators. Perturbing $T_{0}$ by $L$ and $\boldsymbol{T}_{0}$ by $\boldsymbol{L}$ yields $\mathcal{C}_{0}$-semigroups $T$ on $X$ and $\boldsymbol{T}$ on $\boldsymbol{X}$, respectively. Let $A$ and $\boldsymbol{A}$ be their generators. 
Remark 6 . There are at least two equivalent ways to compute $\boldsymbol{T}$ and $\boldsymbol{A}$ on $\boldsymbol{X}$ and their weak ${ }^{\star}$ coun- $^{-}$ terparts $\boldsymbol{T}^{\odot \star}$ and $\boldsymbol{A}^{\odot \star}$ on $\boldsymbol{X}^{\odot \star}$. One approach - suggested to us by Odo Diekmann - uses integrated semigroup theory to calculate first $\boldsymbol{T}$ and next $\boldsymbol{T}^{\odot \star}$. Then $\boldsymbol{A}^{\odot \star}$ and $\boldsymbol{A}$ are calculated, in that order.

Here we go the other way around: We start by calculating $\boldsymbol{A}^{\odot \star}$ and use it to obtain $\boldsymbol{T}^{\odot \star}$. If desired $\boldsymbol{A}$ and $\boldsymbol{T}$ can then be found by application of (36) and its inverse. This approach is more elementary - we use only theory that was already introduced in Section 2.1 - and it yields the same outcome, as it should.

Proposition 7. The weak $k^{\star}$ generator $\boldsymbol{A}^{\odot \star}$ of $\boldsymbol{T}^{\odot \star}$ has the representation

$$
\mathcal{D}\left(\boldsymbol{A}^{\odot \star}\right)=\mathcal{D}\left(A^{\odot \star}\right) \times \mathbb{K}^{p}, \quad \boldsymbol{A}^{\odot \star}=\left(\begin{array}{cc}
A^{\odot \star} & L_{p} \\
0 & 0
\end{array}\right),
$$

where $A^{\odot \star}$ is the weak $k^{\star}$ generator of $T^{\odot \star}$.

Proof. According to the general theory of Section 2.1 and (4) in particular, we have

$$
\mathcal{D}\left(\boldsymbol{A}^{\odot \star}\right)=\mathcal{D}\left(\boldsymbol{A}_{0}^{\odot \star}\right), \quad \boldsymbol{A}^{\odot \star}=\boldsymbol{A}_{0}^{\odot \star}+\boldsymbol{L} \boldsymbol{j}^{-1},
$$

From (35) we see that

$$
\mathcal{D}\left(\boldsymbol{A}_{0}^{\odot \star}\right)=\mathcal{D}\left(A_{0}^{\odot \star}\right) \times \mathbb{K}^{p}, \quad \boldsymbol{A}_{0}^{\odot \star}=\operatorname{diag}\left(A_{0}^{\odot \star}, 0\right) .
$$

Using (36) and (37) we calculate

$$
\boldsymbol{A}_{0}^{\odot \star}+\boldsymbol{L j}^{-1}=\left(\begin{array}{cc}
A_{0}^{\odot \star} & 0 \\
0 & 0
\end{array}\right)+\left(\begin{array}{cc}
L & L_{p} \\
0 & 0
\end{array}\right)\left(\begin{array}{cc}
j^{-1} & 0 \\
0 & I_{p}
\end{array}\right)
$$

and the result follows.

Lemma 8. Let $L_{p}^{\odot}: X^{\odot} \rightarrow \mathbb{K}^{p}$ be the restriction of $L_{p}^{\star}$ to $X^{\odot}$. Then $L_{p}^{\odot \star}=L_{p}$.

Proof. We begin by noting that - strictly speaking - this statement involves two canonical identifications. Namely, let $i: X^{\odot} \rightarrow X^{\odot \star \star}$ and $i_{p}: \mathbb{K}^{p} \rightarrow \mathbb{K}^{p^{\star \star}}$ be the canonical injection and bijection, respectively. Then $L_{p}^{\odot}:=L_{p}^{\star} i$ and we need to prove that $L_{p}^{\odot \star} i_{p}=L_{p}$. For this it is not difficult to show that

$$
\left\langle L_{p}^{\odot \star} i_{p} \alpha, \varphi^{\odot}\right\rangle=\left\langle L_{p} \alpha, \varphi^{\odot}\right\rangle
$$

for all $\alpha \in \mathbb{K}^{p}$ and for all $\varphi^{\odot} \in X^{\odot}$.

For the purpose of notation, we define the integrated semigroup $W^{\odot \star}$ for $T^{\odot \star}$ as

$$
W^{\odot \star}(t) \varphi^{\odot \star}:=\int_{0}^{t} T^{\odot \star}(\tau) \varphi^{\odot \star} d \tau, \quad t \geq 0 .
$$

with on the right a weak ${ }^{\star}$ Riemann integral of the same type as the integral in (3).

Proposition 9. The semigroup $\boldsymbol{T}^{\odot \star}$ that is weakly ${ }^{\star}$ generated by $\boldsymbol{A}^{\odot \star}$ has the representation

$$
\boldsymbol{T}^{\odot \star}(t)=\left(\begin{array}{cc}
T^{\odot \star}(t) & W^{\odot \star}(t) L_{p} \\
0 & I_{p}
\end{array}\right), \quad t \geq 0 .
$$

Proof. We define a one-parameter family $\boldsymbol{S}$ of bounded linear operators on $\boldsymbol{X}^{\odot}$ by

$$
\boldsymbol{S}(t)=\left(\begin{array}{cc}
T \odot(t) & 0 \\
L_{p}^{\odot} W^{\odot}(t) & I_{p}
\end{array}\right), \quad t \geq 0
$$


where

$$
W^{\odot}(t) \varphi^{\odot}:=\int_{0}^{t} T^{\odot}(\tau) \varphi^{\odot} d \tau, \quad t \geq 0 .
$$

It is easy to check that $\boldsymbol{S}$ is a $\mathcal{C}_{0}$-semigroup on $\boldsymbol{X}^{\odot}$. By Lemma 8 the adjoint semigroup $\boldsymbol{S}^{\star}(t)$ equals the right-hand side of (39) for all $t \geq 0$. We will show that the weak ${ }^{\star}$ generator $\boldsymbol{A}^{\odot \star}$ of $\boldsymbol{T}^{\odot \star}$ is also the weak ${ }^{\star}$ generator of $\boldsymbol{S}^{\star}$. This will then imply that $\boldsymbol{T}^{\odot \star}=\boldsymbol{S}^{\star}$.

We use Proposition 7. Let $\boldsymbol{C}$ be the generator of $\boldsymbol{S}$, so $\boldsymbol{C}^{\star}$ is the weak ${ }^{\star}$ generator of $\boldsymbol{S}^{\star}$. For any $\left(\varphi^{\odot \star}, \alpha\right)$ in $\boldsymbol{X}^{\odot \star}$ and any $t>0$ we have

$$
\frac{1}{t}\left(\boldsymbol{S}^{\star}(t)\left(\varphi^{\odot \star}, \alpha\right)-\left(\varphi^{\odot \star}, \alpha\right)\right)=\frac{1}{t}\left(\begin{array}{c}
T^{\odot \star}(t) \varphi^{\odot \star}-\varphi^{\odot \star} \\
0
\end{array}\right)+\frac{1}{t}\left(\begin{array}{c}
W^{\odot \star}(t) L_{p} \alpha \\
0
\end{array}\right) .
$$

We note that $t^{-1} W^{\odot \star}(t) L_{p} \alpha \rightarrow L_{p} \alpha$ weakly ${ }^{\star}$ as $t \downarrow 0$. It follows that the right-hand side converges weakly ${ }^{\star}$ if and only if $\varphi^{\odot \star} \in D\left(A^{\odot \star}\right)$ and in that case the weak ${ }^{\star}$-limit equals $\left(A^{\odot \star} \varphi^{\odot \star}+L_{p} \alpha, 0\right)=$ $\boldsymbol{A}^{\odot \star}\left(\varphi^{\odot \star}, \alpha\right)$. We conclude that $\boldsymbol{C}^{\star}=\boldsymbol{A}^{\odot \star}$.

\subsection{Spectral theory and the center eigenspace}

Let $T_{0}$ be a $\mathcal{C}_{0}$-semigroup on a complex Banach space $X$ that is sun-reflexive with respect to $T_{0}$. For the purpose of spectral theory, we explicitly take $\mathbb{C}$ as the underlying scalar field. In examples, $X$ will often be a complexification of a real Banach space, see Remark 1.

We are interested in a description of the spectrum and the corresponding (generalized) eigenspaces of the generator $\boldsymbol{A}$ of $\boldsymbol{T}$. In particular, Propositions 11 and 14 below guarantee, respectively, the existence and smooth parametrization of the parameter-dependent local center manifold in Section 3.5.

Proposition 10. The spectrum $\sigma\left(\boldsymbol{A}^{\odot \star}\right)=\sigma\left(A^{\odot \star}\right) \cup\{0\}$ with resolvent operator

$$
R_{z}\left(\boldsymbol{A}^{\odot \star}\right)=\left(\begin{array}{cc}
R_{z}\left(A^{\odot \star}\right) & z^{-1} R_{z}\left(A^{\odot \star}\right) L_{p} \\
0 & z^{-1} I_{p}
\end{array}\right)
$$

for every $z$ in the resolvent set $\rho\left(\boldsymbol{A}^{\odot \star}\right)$.

Proof. From Proposition 7 we have

$$
z I-\boldsymbol{A}^{\odot \star}=\left(\begin{array}{cc}
z I-A^{\odot \star} & -L_{p} \\
0 & z I_{p}
\end{array}\right) .
$$

This upper triangular operator matrix has a bounded inverse if and only if both entries on its diagonal have bounded inverses, which happens if and only if $z \in \rho\left(A^{\odot *}\right)$ and $z \neq 0$. In that case, the inverse is given precisely by the stated expression for $R_{z}\left(\boldsymbol{A}^{\odot \star}\right):=\left(z I-\boldsymbol{A}^{\odot \star}\right)^{-1}$.

In addition we assume that $T_{0}$ is eventually compact and the perturbation $L$ in (37) is compact. As a consequence, the spectral analysis of $\boldsymbol{A}^{\odot \star}$ reduces to an analysis of the poles of its resolvent operator [19, Corollary V.3.2], [58, Section V.10].

Proposition 11. $\boldsymbol{T}$ is an eventually compact $\mathcal{C}_{0}$-semigroup.

Proof. The eventual compactness of $T_{0}$, the finite rank of $I_{p}$ and (34) together imply that $\boldsymbol{T}_{0}$ is eventually compact. Since $L_{p}$ has finite rank and $L$ is compact by assumption, it follows from (37) that $\boldsymbol{L}$ is compact, so $\boldsymbol{T}$ is eventually compact by [11, Theorem 2.8].

Theorem 12. The generalized eigenspace corresponding to $\lambda \in \sigma\left(\boldsymbol{A}^{\odot \star}\right)$ is given by

$$
\mathcal{M}_{\lambda}\left(\boldsymbol{A}^{\odot \star}\right)= \begin{cases}\mathcal{M}_{\lambda}\left(A^{\odot \star}\right) \times\{0\}, & \text { if } \lambda \neq 0, \\ \mathcal{M}_{0}\left(A^{\odot \star}\right) \times\{0\} \oplus\left\{\left(\Gamma_{0} L_{p} \alpha, \alpha\right): \alpha \in \mathbb{C}^{p}\right\}, & \text { if } \lambda=0,\end{cases}
$$

where $\Gamma_{0}$ is a bounded linear operator on $X^{\odot \star}$ mapping into $X^{\odot \odot}$. 
Proof. Let $\lambda \in \sigma\left(\boldsymbol{A}^{\odot \star}\right)$ be arbitrary. Taking residues at $z=\lambda$ in (40), we obtain

$$
\boldsymbol{P}_{\lambda}^{\odot \star}=\left(\begin{array}{cc}
P_{\lambda}^{\odot \star} & \Gamma_{\lambda} L_{p} \\
0 & I_{p} \delta_{\lambda}
\end{array}\right), \quad \Gamma_{\lambda}:=\operatorname{Res}_{z=\lambda} z^{-1} R_{z}\left(A^{\odot \star}\right),
$$

where $\delta_{\lambda}:=\delta_{\lambda, 0}$ is the Kronecker delta and $\boldsymbol{P}_{\lambda}^{\odot \star}$ and $P_{\lambda}^{\odot \star}$ are the spectral projectors corresponding to $\lambda$ for $\boldsymbol{A}^{\odot \star}$ and $A^{\odot \star}$. (If $\lambda$ is in the resolvent set of the respective operator, then the residue - hence the spectral projector - is identically zero.) $\Gamma_{\lambda}$ is pointwise equal to a contour integral with an integrand in the closed subspace $X^{\odot \odot}$ of $X^{\odot \star}$, so $\Gamma_{\lambda}$ maps into $X^{\odot \odot}$.

We will now calculate the range of $\boldsymbol{P}_{\lambda}^{\odot \star}$ from (41). In general,

$$
\mathcal{M}_{\lambda}\left(\boldsymbol{A}^{\odot \star}\right)=\left\{\left(\begin{array}{c}
P_{\lambda}^{\odot \star} \varphi^{\odot \star} \\
0
\end{array}\right)+\left(\begin{array}{c}
\Gamma_{\lambda} L_{p} \alpha \\
\alpha \delta_{\lambda}
\end{array}\right):\left(\varphi^{\odot \star}, \alpha\right) \in \boldsymbol{X}^{\odot \star}\right\} .
$$

First we assume that $\lambda \neq 0$, so $\delta_{\lambda}=0$. We are going to show that

$$
\left\{P_{\lambda}^{\odot \star} \varphi^{\odot \star}+\Gamma_{\lambda} L_{p} \alpha:\left(\varphi^{\odot \star}, \alpha\right) \in \boldsymbol{X}^{\odot \star}\right\}=\mathcal{M}_{\lambda}\left(A^{\odot \star}\right) .
$$

Together with (42) this will then prove the theorem for $\mathcal{M}_{\lambda}\left(\boldsymbol{A}^{\odot \star}\right)$. To verify (43) let $p \in \mathbb{N}$ be the order of $\lambda$ as a pole of $R_{z}\left(A^{\odot \star}\right)$. For $n=1, \ldots, p$ let $B_{n}$ be the coefficient of $(z-\lambda)^{-n}$ in the Laurent series for $R_{z}\left(A^{\odot *}\right)$. A small computation shows that

$$
\Gamma_{\lambda}=\sum_{k=1}^{p}(-1)^{k+1} \lambda^{-k} B_{k}
$$

From [58, Section V.10] we recall the relation $B_{n+1}=\left(A^{\odot \star}-\lambda I\right)^{n} B_{1}$ for all $n \in \mathbb{N}$. Since $B_{1}=P_{\lambda}^{\odot \star}$ and its range $\mathcal{M}_{\lambda}\left(A^{\odot \star}\right)$ is an invariant subspace of $A^{\odot \star}$, this relation implies that $B_{k}$ takes values in $\mathcal{M}_{\lambda}\left(A^{\odot \star}\right)$ for all $k=1, \ldots, p$, so the same is true for $\Gamma_{\lambda}$ by (44). From this it follows that (43) holds.

For the remaining case $\lambda=0$ we have $\delta_{\lambda}=1$, so from (42) we get the direct sum

$$
\mathcal{M}_{0}\left(\boldsymbol{A}^{\odot \star}\right)=\left\{\left(\begin{array}{c}
P_{0}^{\odot \star} \varphi^{\odot \star} \\
0
\end{array}\right): \varphi^{\odot \star} \in X^{\odot \star}\right\} \oplus\left\{\left(\begin{array}{c}
\Gamma_{0} L_{p} \alpha \\
\alpha
\end{array}\right): \alpha \in \mathbb{C}^{p}\right\} .
$$

The first summand equals $\mathcal{M}_{0}\left(A^{\odot \star}\right) \times\{0\}$ and this gives the result.

Corollary 13. The center eigenspace $\boldsymbol{X}_{0}$ corresponding to the purely imaginary eigenvalues of $\boldsymbol{A}$ is given by

$$
\boldsymbol{X}_{0}=X_{0} \times\{0\} \oplus\left\{\left(j^{-1} \Gamma_{0} L_{p} \alpha, \alpha\right): \alpha \in \mathbb{C}^{p}\right\},
$$

with $\operatorname{dim} \boldsymbol{X}_{0}=\operatorname{dim} X_{0}+p$.

Proof. By Proposition 10 we have the disjoint union $\sigma\left(\boldsymbol{A}^{\odot \star}\right)=\left(\sigma\left(A^{\odot \star}\right) \backslash\{0\}\right) \cup\{0\}$. Using this and Theorem 12 we first compute the center eigenspace for $\boldsymbol{A}^{\odot \star}$ as

$$
\boldsymbol{X}_{0}^{\odot \star}=X_{0}^{\odot \star} \times\{0\} \oplus\left\{\left(\Gamma_{0} L_{p} \alpha, \alpha\right): \alpha \in \mathbb{C}^{p}\right\}
$$

and then we apply $\boldsymbol{j}^{-1}$ from (36) to both sides of this equality.

In Sections 3.4 to 3.6 we will consider nonlinear problems on a real Banach space. In this case spectral analysis must be preceded by complexification, see Remark 1 and in particular [14, last part of Section IV.2].

Proposition 14. Suppose that $X=Y_{\mathbb{C}}$ is a complexification of a real Banach space $Y$ and let $Y_{0} \subseteq Y$ be the real center eigenspace associated with $X_{0}$. Then the real center eigenspace $\boldsymbol{Y}_{0}$ associated with $\boldsymbol{X}_{0}$ is

$$
\boldsymbol{Y}_{0}=Y_{0} \times\{0\} \oplus\left\{(Q \alpha, \alpha): \alpha \in \mathbb{R}^{p}\right\} \subseteq Y \times \mathbb{R}^{p},
$$

where $Q: \mathbb{R}^{p} \rightarrow Y$ is a bounded linear operator. Furthermore, $\iota: \boldsymbol{Y}_{0} \rightarrow Y_{0} \times \mathbb{R}^{p}$ defined by $\iota(\psi, \alpha):=$ $(\psi-Q \alpha, \alpha)$ is a linear isomorphism. 
Proof. $\boldsymbol{X}$ is naturally identified with $\boldsymbol{Y}_{\mathbb{C}}$ where $\boldsymbol{Y}=Y \times \mathbb{R}^{p}$. Let $\boldsymbol{P}_{\Lambda}$ with range $\boldsymbol{X}_{0}$ be the spectral projector on $\boldsymbol{X}$ for the spectral set $\Lambda=\bar{\Lambda}$ of all purely imaginary eigenvalues of $\boldsymbol{A}_{\mathbb{C}}$. A direct generalization of [14, Corollary IV.2.19] implies that $\boldsymbol{P}_{\Lambda}$ is the complexification of a projector $\boldsymbol{P}_{\Lambda}^{Y}$ on $\boldsymbol{Y}$ and the range $\boldsymbol{Y}_{0}$ of $\boldsymbol{P}_{\Lambda}^{Y}$ - identified with a subspace of $\boldsymbol{Y}$ - is the real center eigenspace for $\boldsymbol{A}$. Also, $\Gamma_{0}$ on $X^{\odot \star}$ is self-conjugate by (41). Together with Corollary 13 this implies (45). It is easily verified that the linear operator $\iota$ is an isomorphism.

Remark 15. We will not make a notational distinction between the real and complex center eigenspaces, indicating both $X_{0}$ and $Y_{0}$ with $X_{0}$ and both $\boldsymbol{X}_{0}$ and $\boldsymbol{Y}_{0}$ with $\boldsymbol{X}_{0}$, respectively. We hope that the underlying scalar field will be clear from the immediate context.

\subsection{Nonlinear perturbation}

Let $T_{0}$ be a $\mathcal{C}_{0}$-semigroup on a real Banach space $X$ that is sun-reflexive with respect to $T_{0}$. Introduce a nonlinear perturbation $\boldsymbol{R}: \boldsymbol{X} \rightarrow \boldsymbol{X}^{\odot \star}$ of the form

$$
\boldsymbol{R}(\varphi, \alpha)=(R(\varphi, \alpha), 0),
$$

where $R: X \rightarrow X^{\odot *}$ is $C^{k}$-smooth, satisfying

$$
R(0,0)=0, \quad D_{1} R(0,0)=0, \quad D_{2} R(0,0)=0 .
$$

We associate with $\boldsymbol{T}$ and $\boldsymbol{R}$ the integral equation

$$
\boldsymbol{u}(t)=\boldsymbol{T}(t-s) \boldsymbol{u}(s)+\boldsymbol{j}^{-1} \int_{s}^{t} \boldsymbol{T}^{\odot \star}(t-\tau) \boldsymbol{R}(\boldsymbol{u}(\tau)) d \tau, \quad-\infty<s \leq t<\infty .
$$

We expect all nontrivial dynamics to be contained in the first component, and this is indeed the case:

Proposition 16. The function $\boldsymbol{u}=\left(u, u_{p}\right): I \rightarrow \boldsymbol{X}$ is a solution of (48) if and only if $u_{p}$ is constant on $I$ and $u: I \rightarrow X$ is a solution of

$$
u(t)=T(t-s) u(s)+j^{-1} \int_{s}^{t} T^{\odot \star}(t-\tau)\left(L_{p} \alpha+R(u(\tau), \alpha)\right) d \tau, \quad-\infty<s \leq t<\infty,
$$

where $\alpha \in \mathbb{R}^{p}$ denotes the constant value of $u_{p}$.

Proof. We use Proposition 9 and (36). For any continuous function $\boldsymbol{u}=\left(u, u_{p}\right): I \rightarrow \boldsymbol{X}$ we compute

$$
\boldsymbol{T}(t-s) \boldsymbol{u}(s)=\boldsymbol{j}^{-1} \boldsymbol{T}^{\odot \star}(t-s) \boldsymbol{j} \boldsymbol{u}(s)=\left(\begin{array}{c}
T(t-s) u(s)+j^{-1} W^{\odot \star}(t-s) L_{p} u_{p}(s) \\
u_{p}(s)
\end{array}\right),
$$

while another computation shows that

$$
j^{-1} \int_{s}^{t} \boldsymbol{T}^{\odot \star}(t-\tau) \boldsymbol{R}(\boldsymbol{u}(\tau)) d \tau=\left(\begin{array}{c}
j^{-1} \int_{s}^{t} T^{\odot \star}(t-\tau) R(\boldsymbol{u}(\tau)) d \tau \\
0
\end{array}\right) .
$$

From the above together with the definition $(38)$ of $W^{\odot \star}(t)$ we see that (48) is equivalent to the system

$$
\left\{\begin{aligned}
u(t) & =T(t-s) u(s)+j^{-1} \int_{s}^{t} T^{\odot \star}(t-\tau)\left(L_{p} u_{p}(s)+R\left(u(\tau), u_{p}(\tau)\right)\right) d \tau, \\
u_{p}(t) & =u_{p}(s)
\end{aligned}\right.
$$

for $-\infty<s \leq t<\infty$. The statement now follows. 


\subsection{Parameter-dependent local center manifolds}

We consider again a $\mathcal{C}_{0}$-semigroup $T_{0}$ on a real Banach space $X$ that is sun-reflexive with respect to $T_{0}$. We also assume that $T_{0}$ is eventually compact and $L$ in (37) is compact, so Proposition 11 implies that $\boldsymbol{T}$ is eventually compact. If furthermore the nonlinearity $\boldsymbol{R}$ satisfies (47), then all conditions are fulfilled for the application of the center manifold theory from [14, Chapter IX] to (48).

Therefore, if the generator $A$ of $T$ has $1 \leq n_{0}<\infty$ purely imaginary eigenvalues, counting algebraic multiplicities, then by Proposition 14 the real center eigenspace $\boldsymbol{X}_{0}$ has dimension $n_{0}+p$. There exists a $C^{k}$-smooth local center manifold $\mathcal{W}_{\text {loc }}^{c}$ in $\boldsymbol{X}$ that is tangent at the origin to $\boldsymbol{X}_{0}$. In fact, Proposition 14 implies that $\mathcal{W}_{\text {loc }}^{c}$ is the image of a $C^{k}$-smooth map

$$
\mathcal{C}: U \times U_{p} \subseteq X_{0} \times \mathbb{R}^{p} \rightarrow \boldsymbol{X},
$$

where $U \subseteq X_{0}$ and $U_{p} \subseteq \mathbb{R}^{p}$ are neighborhoods of the origin. Since (46) has a zero in the second component, it follows from [14, (5.1) in Section IX.5] that $\mathcal{C}$ has the form

$$
\mathcal{C}(\varphi, \alpha)=(\mathcal{C}(\varphi, \alpha), \alpha), \quad \forall(\varphi, \alpha) \in U \times U_{p},
$$

where $\mathcal{C}: U \times U_{p} \rightarrow X$ is the first component function.

Definition 17. The image $\mathcal{W}_{\mathrm{loc}}^{c}(\alpha):=\mathcal{C}(U, \alpha)$ is a local center manifold for (49) at $\alpha \in U_{p}$.

It is a direct consequence of the above definition that for every $\alpha \in U_{p}$ we can parametrize $\mathcal{W}_{\text {loc }}^{c}(\alpha)$ by coordinates on the real center eigenspace $X_{0}$ that depend $C^{k}$-smoothly on $\alpha$. This will be important for the discussion of the normalization method following (55) in Section 4.

Proposition 18. If $\alpha \in U_{p}$ is sufficiently small then $\mathcal{W}_{\text {loc }}^{c}(\alpha)$ is locally positively invariant for the semiflow generated by (49).

Proof. Let $\boldsymbol{\Sigma}$ and $\Sigma$ be the semiflows generated by (48) and (49), respectively. By Proposition 16,

$$
\boldsymbol{\Sigma}(s,(\psi, \alpha))=(\Sigma(s, \psi), \alpha), \quad \forall \psi \in X, \alpha \in \mathbb{R}^{p},
$$

and for all $s$ in a common interval of existence $I_{\psi, \alpha}$. By [14, Theorem IX.5.3(i)] there exists $\delta>0$ such that if $(\psi, \alpha) \in \mathcal{W}_{\text {loc }}^{c}$ and if

$$
\|\boldsymbol{\Sigma}(s,(\psi, \alpha))\|=\|\Sigma(s, \psi)\|+|\alpha| \leq \delta, \quad \forall s \in[0, t],
$$

then $\boldsymbol{\Sigma}(t,(\psi, \alpha)) \in \mathcal{W}_{\text {loc }}^{c}$ which by (51) implies that $\Sigma(t, \psi) \in \mathcal{W}_{\text {loc }}^{c}(\alpha)$.

We note that if $\psi \in \mathcal{W}_{\text {loc }}^{c}(\alpha)$ then by (50) it follows that $(\psi, \alpha) \in \mathcal{W}_{\text {loc }}^{c}$. Therefore, if $|\alpha| \leq \frac{\delta}{2}$ and $\psi \in \mathcal{W}_{\mathrm{loc}}^{c}(\alpha)$ then

$$
\|\Sigma(s, \psi)\| \leq \frac{\delta}{2}, \quad \forall s \in[0, t]
$$

implies that $\Sigma(t, \psi) \in \mathcal{W}_{\text {loc }}^{c}(\alpha)$. This is precisely local positive invariance of $\mathcal{W}_{\text {loc }}^{c}(\alpha)$ for $\Sigma$.

Next we consider a solution $u: I \rightarrow X$ of (49) that lies in $\mathcal{W}_{\text {loc }}^{c}(\alpha)$. By Proposition 16 the function $\boldsymbol{u}=(u, \alpha): I \rightarrow \boldsymbol{X}$ is a solution of (48). Also, since $u$ lies in $\mathcal{W}_{\text {loc }}^{c}(\alpha)$ we see from (50) that $\boldsymbol{u}$ lies in $\mathcal{W}_{\text {loc }}^{c}$ and therefore satisfies the differential equation

$$
\boldsymbol{j} \boldsymbol{u}(t)=\boldsymbol{A}^{\odot \star} \boldsymbol{j} \boldsymbol{u}(t)+\boldsymbol{R}(\boldsymbol{u}(t)), \quad \forall t \in I,
$$

cf. (9). By (36) and (46) and Proposition 7 the first component of this equation gives the differential equation

$$
j \dot{u}(t)=A^{\odot \star} j u(t)+L_{p} \alpha+R(u(t), \alpha), \quad \forall t \in I,
$$

that is satisfied by $u$.

In summary, 
Theorem 19 (Parameter-dependent local center manifold). Let $T_{0}$ be an eventually compact $\mathcal{C}_{0^{-}}$ semigroup on a sun-reflexive real Banach space $X$ and let $T$ be the $\mathcal{C}_{0}$-semigroup on $X$ defined by (3) where $L$ is a compact perturbation. Suppose that the generator $A$ of $T$ has $1 \leq n_{0}<\infty$ purely imaginary eigenvalues with corresponding $n_{0}$-dimensional real center eigenspace $X_{0}$. Furthermore, assume that $R$ in (49) is $C^{k}$-smooth and (47) holds.

Then there exists a $C^{k}$-smooth map $\mathcal{C}: U \times U_{p} \rightarrow X$ defined in a neighborhood of the origin in $X_{0} \times \mathbb{R}^{p}$ and such that for every sufficiently small $\alpha \in \mathbb{R}^{p}$ the manifold $\mathcal{W}_{\text {loc }}^{c}(\alpha):=\mathcal{C}(U, \alpha)$ is locally positively invariant for the semiflow generated by (49) at parameter value $\alpha$. Furthermore, any solution $u: I \rightarrow X$ of (49) that lies on $\mathcal{W}_{\text {loc }}^{c}(\alpha)$ satisfies (52).

\subsection{The special case of parameter-dependent classical DDEs}

In this section we will formulate a corollary of Theorem 19 that applies specifically to the classical parameter-dependent DDE (28). As in Section 2.4 our starting point is (32) with $F=0$,

$$
\left\{\begin{array}{l}
\dot{x}(t)=0, \\
\dot{\mu}(t)=0,
\end{array} \quad t \geq 0,\right.
$$

in the unknown $(x, \mu)$ with initial condition $(\varphi, \alpha)$ in the state space $\boldsymbol{X}:=X \times \mathbb{R}^{p}$ where $X:=$ $C\left([-h, 0], \mathbb{R}^{n}\right)$. So, we interpret the first component of (53) as a DDE but the second component as an ODE. By comparison with (13) it is clear that the solution of the initial value problem for (53) defines a $\mathcal{C}_{0}$-semigroup $\boldsymbol{T}_{0}$ on $\boldsymbol{X}$,

$$
\boldsymbol{T}_{0}(t):=\operatorname{diag}\left(T_{0}(t), I_{p}\right)
$$

with $T_{0}$ the eventually compact shift semigroup on $X$ from (14) and $I_{p}$ the identity on $\mathbb{R}^{p}$. We specify the perturbations $L$ and $L_{p}$ in (37) as

$$
L \varphi=\left(D_{1} F(0,0) \varphi\right) r^{\odot \star}, \quad L_{p} \alpha=\left(D_{2} F(0,0) \alpha\right) r^{\odot \star}
$$

where $D_{1} F(0,0)$ and $D_{2} F(0,0)$ are as in (31). Then $L$ is of finite rank, so certainly it is compact. Also, we choose the nonlinear perturbation $R$ in (46) as

$$
R(\varphi, \alpha)=G(\varphi, \alpha) r^{\odot *}
$$

where $G$ is defined by the splitting in (31). Then (33) implies that the conditions in (47) hold.

Corollary 20 (Parameter-dependent local center manifold for DDEs). Consider the particular case of the classical DDE in (28),

$$
\dot{x}(t)=F\left(x_{t}, \alpha\right), \quad t \geq 0,
$$

where $F: X \times \mathbb{R}^{p} \rightarrow \mathbb{R}^{n}$ is $C^{k}$-smooth for some $k \geq 1$ with $F(0,0)=0$. Let $T$ be the $\mathcal{C}_{0}$-semigroup on $X$ corresponding to the linearization of (28) at $0 \in X$ for the critical parameter value $\alpha=0$. Suppose that the generator $A$ of $T$ has $1 \leq n_{0}<\infty$ purely imaginary eigenvalues with corresponding $n_{0}$-dimensional real center eigenspace $X_{0}$.

Then there exists a $C^{k}$-smooth map $\mathcal{C}: U \times U_{p} \rightarrow X$ defined in a neighborhood of the origin in $X_{0} \times \mathbb{R}^{p}$ and such that for every sufficiently small $\alpha \in \mathbb{R}^{p}$ the manifold $\mathcal{W}_{\text {loc }}^{c}(\alpha):=\mathcal{C}(U, \alpha)$ is locally positively invariant for the semiflow generated by (28) at parameter value $\alpha$.

Furthermore, if the history $x_{t}$ associated with a solution of (28) exists on some nondegenerate interval $I$ and $x_{t} \in \mathcal{W}_{\text {loc }}^{c}(\alpha)$ for all $t \in I$, then $u: I \rightarrow X$ defined by $u(t):=x_{t}$ is differentiable and satisfies

$$
j \dot{u}(t)=A^{\odot \star} j u(t)+\left(D_{2} F(0,0) \alpha\right) r^{\odot \star}+G(u(t), \alpha) r^{\odot \star}, \quad \forall t \in I,
$$

where $A^{\odot \star}$ is the weak $k^{\star}$ generator of $T^{\odot \star}$ and the operator $G: X \times \mathbb{R}^{p} \rightarrow \mathbb{R}^{n}$ defined by (31),

$$
G(\varphi, \alpha):=F(\varphi, \alpha)-D_{1} F(0,0) \varphi-D_{2} F(0,0) \alpha,
$$

is the nonlinear part of $F$. 
Remark 21. For discrete classical DDEs (1) one may want to use one or more of the discrete delays $\tau_{1}, \ldots, \tau_{m}$ as parameters. However, in this case the operator $R$ is typically no longer differentiable, see [14, Remark IX.9.2]. Of course, if only a single discrete delay is present, this problem can be avoided by a linear rescaling of time.

\section{Normal forms on the parameter-dependent center manifold}

The normalization technique described in this section goes back to [8]. In [42] it was applied to obtain expressions for the critical normal form coefficients of all generic codimension one and two bifurcations of equilibria in ODEs, also see $[43, \S 8.7]$. In this context, these expressions are independent of the (finite) dimension of the phase space and they involve only critical eigenvectors of the Jacobian matrix and its transpose as well as higher order derivatives of the right-hand side at the critical equilibrium. These properties make them suitable for both symbolic and numerical evaluation.

In [46] the same technique was applied to parameter-dependent normal forms to start the continuation of nonhyperbolic cycles emanating from generalized Hopf, fold-Hopf and Hopf-Hopf bifurcation points of ODEs. The resulting predictors were implemented in the freely available software package MatCont, a MATLAB toolbox for continuation and bifurcation analysis of finite dimensional dynamical systems. This makes it possible to verify transversality conditions and to initialize the continuation of the nonhyperbolic cycles mentioned above. A similar switching problem for iterated maps was solved earlier in [27].

In [37] the normalization technique was lifted to an infinite dimensional setting, providing explicit expressions for the critical normal form coefficients of generic codimension one and two equilibrium bifurcations in DDEs. These expressions were partially implemented [60] in the software DDE-BifTool. This package can be considered as the DDE equivalent of MatCont in command line mode.

In this section we extend the normalization method from [37] to include parameters. Suppose $0 \in X$ is a stationary state of (28) at the critical parameter value $0 \in \mathbb{R}^{p}$ and assume there are $n_{0} \geq 1$ eigenvalues on the imaginary axis, counting algebraic multiplicities. Let $P_{0}$ be the corresponding real spectral projector on $X$, so the range $X_{0}$ of $P_{0}$ is the real $n_{0}$-dimensional center eigenspace. Corollary 20 applies to give a parameter-dependent local center manifold $\mathcal{W}_{\text {loc }}^{c}(\alpha)$ for $(28)$.

We allow for the introduction of a new parameter $\beta$ defined in a neighborhood of $0 \in \mathbb{R}^{p}$ such that $\alpha=K(\beta)$ for some locally defined $C^{k}$-diffeomorphism $K: \mathbb{R}^{p} \rightarrow \mathbb{R}^{p}$ that is to be determined below, up to a certain order. If $u: I \rightarrow X$ with $u(t):=x_{t} \in \mathcal{W}_{\text {loc }}^{c}(\alpha)$ is as in Corollary 20, then $u$ is differentiable on $I$ and satisfies

$$
j \dot{u}(t)=A^{\odot \star} j u(t)+\left(D_{2} F(0,0) K(\beta)\right) r^{\odot \star}+R(u(t), K(\beta)), \quad \forall t \in I,
$$

where $R$ encodes the nonlinear part of $F$ as in (54). Choose a basis $\Phi$ of $X_{0}$ and let $\mathcal{H}: \mathbb{R}^{n_{0}} \times \mathbb{R}^{p} \rightarrow X$ be a locally defined $C^{k}$-smooth parametrization of $\mathcal{W}_{\text {loc }}^{c}(\alpha)$ with respect to $\Phi$ and in terms of the new parameter $\beta$, see the remark following Definition 17. For every $t \in I$ we define $v(t) \in \mathbb{R}^{n_{0}}$ as the coordinate vector of $P_{0} u(t)$ with respect to $\Phi$. Then $v: I \rightarrow \mathbb{R}^{n_{0}}$ satisfies a parameter-dependent ordinary differential equation of the form

$$
\dot{v}=\sum_{|\nu|+|\mu| \geq 1} \frac{1}{\nu ! \mu !} g_{\nu \mu} v^{\nu} \beta^{\mu},
$$

where the $C^{k}$-smooth vector field on the right has been expanded up to some sufficiently large - but finite - order. The multi-indices $\nu$ and $\mu$ have lengths $n_{0}$ and $p$, respectively. We assume that (56) is in parameter-dependent normal form, up to a certain order. Since $\mathcal{H}$ parametrizes $\mathcal{W}_{\text {loc }}^{c}(\alpha)$,

$$
u(t)=\mathcal{H}(v(t), \beta), \quad t \in I,
$$


with both $u$ and $v$ depending on the parameter, although this is left implicit in the notation. Substituting the above relation into (55) produces the homological equation

$$
A^{\odot \star} j \mathcal{H}(v, \beta)+\left(D_{2} F(0,0) K(\beta)\right) r^{\odot \star}+R(\mathcal{H}(v, \beta), K(\beta))=j D_{1} \mathcal{H}(v, \beta) \dot{v},
$$

with $\dot{v}$ given by the parameter-dependent normal form (56). The unknowns in (HOM) are $\mathcal{H}, K$ and the coefficients $g_{\nu \mu}$ from (56). They are determined, up to a certain order, by the assumption that (56) is in normal form. For $r, s \geq 0$ with $r+s \geq 1$ we denote by $D_{1}^{r} D_{2}^{s} F(0,0): X^{r} \times\left[\mathbb{R}^{p}\right]^{s} \rightarrow \mathbb{R}^{n}$ the mixed Fréchet derivative of order $r+s$, evaluated at $(0,0) \in X \times \mathbb{R}^{p}$, with the understanding that at most one of the factor spaces $X^{r}$ or $\left[\mathbb{R}^{p}\right]^{s}$ is absent if either $r=0$ or $s=0$. We expand the nonlinearity $R$ as

$$
R(\varphi, \alpha)=\sum_{r+s>1} \frac{1}{r ! s !} D_{1}^{r} D_{2}^{s} F(0,0)\left(\varphi^{(r)}, \alpha^{(s)}\right) r^{\odot \star},
$$

where $\varphi^{(r)}:=(\varphi, \ldots, \varphi) \in X^{r}$ and $\alpha^{(s)}:=(\alpha, \ldots, \alpha) \in\left[\mathbb{R}^{p}\right]^{s}$. The mappings $\mathcal{H}$ and $K$ can be expanded as

$$
\mathcal{H}(v, \beta)=\sum_{|\nu|+|\mu| \geq 1} \frac{1}{\nu ! \mu !} H_{\nu \mu} v^{\nu} \beta^{\mu}, \quad K(\beta)=\sum_{|\mu| \geq 1} \frac{1}{\mu !} K_{\mu} \beta^{\mu} .
$$

Substituting (56)-(58) into (HOM), collecting coefficients of terms $v^{\nu} \beta^{\mu}$ from lower to higher order and solving the resulting linear systems, one can solve recursively for the unknown coefficients $g_{\nu \mu}, H_{\nu \mu}$ and $K_{\mu}$ by applying the Fredholm alternative and taking ordinary or bordered inverses, as explained in Section 2.6.

\section{Predictors for normal forms on center manifolds}

Starting from this point, we will focus exclusively on two-parameter DDEs, i.e. we will have $p=2$. This enables the initialization of codimension one equilibrium and cycle bifurcations emanating from the generalized Hopf, fold-Hopf, Hopf-Hopf and transcritical-Hopf codimension two bifurcation points. The corresponding steady state and cycle bifurcation curves can then be continued in two parameters using the continuation capabilities of DDE-BifTool, providing an unfolding of the singularity.

In this section we list known asymptotics for codimension one nonhyperbolic cycles emanating from generalized Hopf, fold-Hopf and Hopf-Hopf bifurcations obtained in [46]. The asymptotics are derived from the Poincaré normal forms which are obtained by considering near-identity changes of coordinates generated by homogeneous polynomial functions without using time reparametrization. These normal forms are therefore ready to be used in conjunction with the homological equation (HOM) where all the time derivatives are assumed to be with the same unit of time. Following the same method as in [46], we also derive asymptotics for codimension one nonhyperbolic cycles emanating from the transcritical-Hopf bifurcation. We also provide asymptotics for the codimension one equilibrium bifurcations emanating from the degenerate Hopf points.

\subsection{Generalized-Hopf bifurcation}

Suppose that system (28) has an equilibrium $\varphi=0$ at the critical parameter value $\alpha_{0}=(0,0) \in \mathbb{R}^{2}$ with purely imaginary eigenvalues

$$
\lambda_{1,2}= \pm i \omega_{0}, \quad \omega_{0}>0 .
$$

Furthermore, suppose that the first Lyapunov coefficient $\ell_{1}(0)=0$ while the second Lyapunov coefficient $\ell_{2}(0) \neq 0$. Then the restriction of $(28)$ to the two-dimensional center manifold $\mathcal{W}_{\text {loc }}^{c}(\alpha)$ can be transformed into the smooth local normal form

$$
\dot{z}=\lambda(\alpha) z+c_{1}(\alpha) z|z|^{2}+c_{2}(\alpha) z|z|^{4}+\mathcal{O}\left(|z|^{6}\right),
$$


where $\lambda(\alpha), c_{1}(\alpha), c_{2}(\alpha)$ are complex-valued functions with $\ell_{1}(0)=\frac{1}{\omega_{0}} \operatorname{Re} c_{1}(0)=0, \lambda(0)=i \omega_{0}$ and $\ell_{2}(0)=\frac{1}{\omega_{0}} \operatorname{Re} c_{2}(0) \neq 0$. Let

$$
\left\{\begin{aligned}
\lambda(\alpha) & =\mu(\alpha)+i \omega(\alpha) \\
c_{1}(\alpha) & =\operatorname{Re} c_{1}(\alpha)+i \operatorname{Im} c_{1}(\alpha),
\end{aligned}\right.
$$

where $\mu(\alpha)$ and $\omega(\alpha)$ are real-valued functions. Suppose that the map $\alpha \mapsto\left(\mu(\alpha), \operatorname{Re} c_{1}(\alpha)\right)$ is regular at $\alpha=0$. Then we can introduce new parameters $\left(\beta_{1}(\alpha), \beta_{2}(\alpha)\right)$ to obtain the normal form

$$
\dot{z}=\left(\beta_{1}+i \omega(\beta)\right) z+\left(\beta_{2}+i \operatorname{Im} c_{1}(\beta)\right) z|z|^{2}+c_{2}(\beta) z|z|^{4}+\mathcal{O}\left(|z|^{6}\right),
$$

where $\omega(0)=\omega_{0}$. For convenience, we abuse notations and write $\omega(\beta)$ and $c_{j}(\beta)$ instead of $\omega(\alpha(\beta))$ and $c_{j}(\alpha(\beta))$, respectively. Similar conventions are adopted in other cases ahead.

It is well known that a curve of fold bifurcation of limit cycles (LPC) emanates from this codimension two point. To approximate this curve we substitute $z=\rho e^{i \psi}$ into $(61)$ and expand $\omega(\beta)=\omega(\beta)=$ $\omega_{0}+\omega_{10} \beta_{1}+\omega_{01} \beta_{2}+\mathcal{O}\left(\left\|\left(\beta_{1}, \beta_{2}\right)\right\|^{2}\right)$. Truncating higher order terms and separating the real and imaginary parts yields the system

$$
\left\{\begin{array}{l}
\dot{\rho}=\rho\left(\beta_{1}+\beta_{2} \rho^{2}+\operatorname{Re}\left(c_{2}(0)\right) \rho^{4}\right), \\
\dot{\psi}=\omega_{0}+\omega_{10} \beta_{1}+\omega_{01} \beta_{2}+\operatorname{Im}\left(c_{1}(0)\right) \rho^{2}+\operatorname{Im}\left(c_{2}(0)\right) \rho^{4} .
\end{array}\right.
$$

The curve LPC corresponds to a double zero in the amplitude equation in (62). Therefore, this curve in (61) can be approximated by

$$
\rho=\epsilon, \quad \beta_{1}=\operatorname{Re}\left(c_{2}(0)\right) \epsilon^{4}, \quad \beta_{2}=-2 \operatorname{Re}\left(c_{2}(0)\right) \epsilon^{2}, \quad \epsilon>0 .
$$

From the second equation in the amplitude system (62) we obtain using equation (63) the following approximation for the period:

$$
T=2 \pi /\left(\omega_{0}+\left(\operatorname{Im}\left(c_{1}(0)\right)-2 \operatorname{Re} c_{2}(0) \omega_{01}\right) \epsilon^{2}\right) .
$$

Since $\operatorname{Re}\left(c_{1}(\beta)\right)=\beta_{2}$, it is easy to see that the Hopf curve in the original system is related to the truncated normal form by

$$
\left(\beta_{1}, \beta_{2}, z\right)=(0, \epsilon, 0)
$$

for $\epsilon \neq 0$ small.

\subsection{Fold-Hopf bifurcation}

Suppose that system (28) has an equilibrium $x=0$ at the critical parameter value $\alpha_{0}=(0,0) \in \mathbb{R}^{2}$ with eigenvalues

$$
\lambda_{1}=0, \quad \lambda_{2,3}= \pm i \omega_{0}
$$

where $\omega_{0}>0$. The restriction of $(28)$ to the three-dimensional center manifold $\mathcal{W}_{\text {loc }}^{c}(\alpha)$ can generically be transformed to the smooth local normal form

$$
\left\{\begin{aligned}
& \dot{z}_{0}= \gamma(\alpha)+g_{200}(\alpha) z_{0}^{2}+g_{011}(\alpha)\left|z_{1}\right|^{2}+g_{300}(\alpha) z_{0}^{3}+g_{111}(\alpha) z_{0}\left|z_{1}\right|^{2} \\
& \quad+\mathcal{O}\left(\left\|\left(z_{0}, z_{1}, \bar{z}_{1}\right)\right\|^{4}\right), \\
& \dot{z}_{1}=\lambda(\alpha) z_{1}+g_{110}(\alpha) z_{0} z_{1}+g_{210}(\alpha) z_{0}^{2} z_{1}+g_{021}(\alpha) z_{1}\left|z_{1}\right|^{2}+\mathcal{O}\left(\left\|\left(z_{0}, z_{1}, \bar{z}_{1}\right)\right\|^{4}\right),
\end{aligned}\right.
$$

where $z_{0} \in \mathbb{R}, z_{1} \in \mathbb{C}, \gamma(0)=0, \lambda(0)=i \omega_{0}$ and the functions $g_{j k l}(\alpha)$ are real in the first equation and complex in the second. Let $\lambda(\alpha)=\mu(\alpha)+i \omega(\alpha)$ and suppose that the map $\alpha \mapsto(\gamma(\alpha), \mu(\alpha))$ is regular at $\alpha=0$. Introducing new parameters $\left(\beta_{1}(\alpha), \beta_{2}(\alpha)\right)$, we obtain the truncated normal form

$$
\left\{\begin{array}{l}
\dot{z}_{0}=\beta_{1}+g_{200}(\beta) z_{0}^{2}+g_{011}(\beta)\left|z_{1}\right|^{2}+g_{111}(\beta) z_{0}\left|z_{1}\right|^{2}+g_{300}(\beta) z_{0}^{3}, \\
\dot{z}_{1}=\left(\beta_{2}+i \omega_{0}+i b_{1}(\beta)\right) z_{1}+g_{110}(\beta) z_{0} z_{1}+g_{210}(\beta) z_{0}^{2} z_{1}+g_{021}(\beta) z_{1}\left|z_{1}\right|^{2},
\end{array}\right.
$$


with $b_{1}(0)=0$. Letting $z_{1}=\rho e^{i \psi}$ and separating the real and imaginary parts yields the system

$$
\left\{\begin{aligned}
\dot{z}_{0} & =\beta_{1}+g_{200}(\beta) z_{0}^{2}+g_{011}(\beta) \rho^{2}+g_{111}(\beta) z_{0} \rho^{2}+g_{300}(\beta) z_{0}^{3}, \\
\dot{\rho} & =\rho\left(\beta_{2}+\operatorname{Re}\left(g_{110}(\beta)\right) z_{0}+\operatorname{Re}\left(g_{210}(\beta)\right) z_{0}^{2}+\operatorname{Re}\left(g_{021}(\beta)\right) \rho^{2}\right), \\
\dot{\psi} & =\omega_{0}+b_{1}(\beta)+\operatorname{Im}\left(g_{110}(\beta)\right) z_{0}+\operatorname{Im}\left(g_{210}(\beta)\right) z_{0}^{2}+\operatorname{Im}\left(g_{021}(\beta)\right) \rho^{2} .
\end{aligned}\right.
$$

\subsubsection{Neimark-Sacker curve}

A Hopf bifurcation in the amplitude system (69), i.e. when the trace of the Jacobian matrix of the amplitude system vanishes but the determinant of this matrix is positive, corresponds to a NeimarkSacker bifurcation in the original system. Let $\rho=\epsilon$ and suppose that $g_{011}(0) \operatorname{Re}\left(g_{110}(0)\right)<0$. Then by the implicit function theorem, we obtain the second-order predictor

$$
\left\{\begin{array}{l}
\beta_{1}=-g_{011}(0) \epsilon^{2} \\
\beta_{2}=\frac{\operatorname{Re}\left(g_{110}(0)\right)\left(2 \operatorname{Re}\left(g_{021}(0)\right)+g_{111}(0)\right)-2 \operatorname{Re}\left(g_{021}(0)\right) g_{200}(0)}{2 g_{200}(0)} \epsilon^{2}, \\
z_{0}=-\frac{2 \operatorname{Re}\left(g_{021}(0)\right)+g_{111}(0)}{2 g_{200}(0)} \epsilon^{2},
\end{array}\right.
$$

for the Neimark-Sacker curve. It follows from (69) that the period of the cycle is approximated to the same order by

$$
T=2 \pi /\left(\omega_{0}+\omega_{1} \beta_{1}+\omega_{2} \beta_{2}+\operatorname{Im}\left(g_{110}(0)\right) z_{0}+\operatorname{Im}\left(g_{021}(0)\right) \epsilon^{2}\right) .
$$

Here $\left(z_{0}, \beta_{1}, \beta_{2}\right)$ are as in $(70)$ and $\omega_{i}=\frac{\partial b_{1}}{\partial \beta_{i}}(0)$, for $i=1,2$.

Remark 22. Notice that the cubic terms $g_{210}(0)$ and $g_{300}(0)$ do not appear in the predictor. However, these coefficients are needed to determine the stability of the two-dimensional torus, see [43, Section 8.5] and (106).

\subsubsection{Fold and Hopf curves}

For the approximation to the fold and Hopf curves it is sufficient to consider the second order terms in $\left(z_{0}, \rho\right)$ in the amplitude system in (69). We obtain three equilibrium solutions given by

$$
\pm\left(\sqrt{-\frac{\beta_{1}}{g_{200}(0)}}, 0\right) \quad \text { and } \quad\left(-\frac{\beta_{2}}{\operatorname{Re}\left(g_{110}(0)\right)}, \frac{\sqrt{-\operatorname{Re}\left(g_{110}(0)\right)^{2} \beta_{1}-g_{200}(0) \beta_{2}^{2}}}{\sqrt{g_{011}(0) \operatorname{Re}\left(g_{110}(0)\right)}}\right) .
$$

It follows that the fold curve is approximated by

$$
\left(\beta_{1}, \beta_{2}\right)=(0, \epsilon)
$$

and the Hopf curve by

$$
\left(\beta_{1}, \beta_{2}\right)=\left(-\frac{g_{200}(0)}{\operatorname{Re}\left(g_{110}(0)\right)^{2}} \epsilon^{2}, \epsilon\right)
$$

for $|\epsilon|$ small.

\subsection{Hopf-Hopf bifurcation}

Suppose that system (28) at the critical parameter value $\alpha_{0}=(0,0) \in \mathbb{R}^{2}$ undergoes two Hopf bifurcations simultaneously. Then the generator $A$ has two pairs of purely imaginary eigenvalues

$$
\lambda_{1,4}= \pm i \omega_{1}(0), \quad \lambda_{2,3}= \pm i \omega_{2}(0)
$$


where $\omega_{i}: \mathbb{R}^{2} \rightarrow \mathbb{C}$ for $i=1,2$ such that $\omega_{1}(0)>\omega_{2}(0)>0$. When no other eigenvalues on the imaginary axis exist, this phenomenon is called the Hopf-Hopf or double-Hopf bifurcation. Assume, furthermore that the nonresonance conditions $k \omega_{1} \neq l \omega_{2}$, with $0<k+l \leq 5$ are satisfied. Then the restriction of (28) to the four-dimensional center manifold $\mathcal{W}_{\mathrm{loc}}^{c}(\alpha)$ can be transformed to the smooth local normal form

$$
\left\{\begin{array}{c}
\dot{z}_{1}=\lambda_{1}(\alpha) z_{1}+g_{2100}(\alpha) z_{1}\left|z_{1}\right|^{2}+g_{1011}(\alpha) z_{1}\left|z_{2}\right|^{2}+g_{3200}(\alpha) z_{1}\left|z_{1}\right|^{4} \\
\quad+g_{2111}(\alpha) z_{1}\left|z_{1}\right|^{2}\left|z_{2}\right|^{2}+g_{1022}(\alpha) z_{1}\left|z_{2}\right|^{4}+\mathcal{O}\left(\left\|z_{1}, \overline{z_{1}}, z_{2}, \overline{z_{2}}\right\|^{6}\right), \\
\dot{z}_{2}=\lambda_{2}(\alpha) z_{2}+g_{1110}(\alpha) z_{2}\left|z_{1}\right|^{2}+g_{0021}(\alpha) z_{2}\left|z_{2}\right|^{2}+g_{2210}(\alpha) z_{2}\left|z_{1}\right|^{4} \\
+g_{1121}(\alpha) z_{2}\left|z_{1}\right|^{2}\left|z_{2}\right|^{2}+g_{0032}(\alpha) z_{2}\left|z_{2}\right|^{4}+\mathcal{O}\left(\left\|z_{1}, \overline{z_{1}}, z_{2}, \overline{z_{2}}\right\|^{6}\right),
\end{array}\right.
$$

where $z_{1,}, z_{2} \in \mathbb{C}^{2}$ and $g_{j k l m} \in \mathbb{C}$. Let

$$
\left\{\begin{array}{l}
\lambda_{1}(\alpha)=\mu_{1}(\alpha)+i \omega_{1}(\alpha) \\
\lambda_{2}(\alpha)=\mu_{2}(\alpha)+i \omega_{2}(\alpha)
\end{array}\right.
$$

and suppose that the map $\alpha \mapsto\left(\mu_{1}(\alpha), \mu_{2}(\alpha)\right)$ is regular at $\alpha=0$. Then we can introduce new parameters $\left(\beta_{1}(\alpha), \beta_{2}(\alpha)\right)=\left(\mu_{1}(\alpha), \mu_{2}(\alpha)\right)$ to obtain the normal form

$$
\left\{\begin{array}{c}
\dot{z}_{1}=\left(\beta_{1}+i \omega_{1}(\beta) z_{1}+g_{2100}(\beta) z_{1}\left|z_{1}\right|^{2}+g_{1011}(\beta) z_{1}\left|z_{2}\right|^{2}+g_{3200}(\beta) z_{1}\left|z_{1}\right|^{4}\right. \\
+g_{2111}(\beta) z_{1}\left|z_{1}\right|^{2}\left|z_{2}\right|^{2}+g_{1022}(\beta) z_{1}\left|z_{2}\right|^{4}+\mathcal{O}\left(\left\|z_{1}, \overline{z_{1}}, z_{2}, \overline{z_{2}}\right\|^{6}\right), \\
\dot{z}_{2}=\left(\beta_{2}+i \omega_{2}(\beta) z_{2}+g_{1110}(\beta) z_{2}\left|z_{1}\right|^{2}+g_{0021}(\beta) z_{2}\left|z_{2}\right|^{2}+g_{2210}(\beta) z_{2}\left|z_{1}\right|^{4}\right. \\
+g_{1121}(\beta) z_{2}\left|z_{1}\right|^{2}\left|z_{2}\right|^{2}+g_{0032}(\beta) z_{2}\left|z_{2}\right|^{4}+\mathcal{O}\left(\left\|z_{1}, \overline{z_{1}}, z_{2}, \overline{z_{2}}\right\|^{6}\right) .
\end{array}\right.
$$

We truncate the normal form to third order

$$
\left\{\begin{array}{l}
\dot{z}_{1}=\left(\beta_{1}+i \omega_{1}+i b_{1}(\beta)\right) z_{1}+g_{2100}(0) z_{1}\left|z_{1}\right|^{2}+g_{1011}(0) z_{1}\left|z_{2}\right|^{2} \\
\dot{z}_{2}=\left(\beta_{2}+i \omega_{2}+i b_{2}(\beta)\right) z_{2}+g_{1110}(0) z_{2}\left|z_{1}\right|^{2}+g_{0021}(0) z_{2}\left|z_{2}\right|^{2}
\end{array}\right.
$$

Letting $\left(z_{1}, z_{2}\right)=\left(\rho_{1} e^{i \psi_{1}}, \rho_{2} e^{i \psi_{2}}\right)$ and separating the real and imaginary parts yields

$$
\left\{\begin{array}{l}
\dot{\rho}_{1}=\rho_{1}\left(\beta_{1}+\operatorname{Re}\left(g_{2100}(0)\right) \rho_{1}^{2}+\operatorname{Re}\left(g_{1011}(0)\right) \rho_{2}^{2}\right), \\
\dot{\rho_{2}}=\rho_{2}\left(\beta_{2}+\operatorname{Re}\left(g_{1110}(0)\right) \rho_{1}^{2}+\operatorname{Re}\left(g_{0021}(0)\right) \rho_{2}^{2}\right), \\
\dot{\psi}_{1}=\omega_{1}+b_{1}(\beta)+\operatorname{Im}\left(g_{2100}(0)\right) \rho_{1}^{2}+\operatorname{Im}\left(g_{1011}(0)\right) \rho_{2}^{2}, \\
\dot{\psi}_{2}=\omega_{2}+b_{2}(\beta)+\operatorname{Im}\left(g_{1110}(0)\right) \rho_{1}^{2}+\operatorname{Im}\left(g_{0021}(0)\right) \rho_{2}^{2} .
\end{array}\right.
$$

\subsubsection{Hopf curves}

There are two semi-trivial equilibria

$$
\left(\rho_{1}, \rho_{2}\right)=\left(\sqrt{-\frac{\beta_{1}}{\operatorname{Re}\left(g_{2100}(0)\right)}}, 0\right), \quad\left(\rho_{1}, \rho_{2}\right)=\left(0, \sqrt{-\frac{\beta_{2}}{\operatorname{Re}\left(g_{0021}(0)\right)}}\right)
$$

of the amplitude system of (73). Translating to the original system provides the Hopf bifurcation curves

$$
H_{1}=\left\{\left(\beta_{1}, \beta_{2}\right): \beta_{1}=0\right\}, \quad \text { and } \quad H_{2}=\left\{\left(\beta_{1}, \beta_{2}\right): \beta_{2}=0\right\} .
$$




\subsubsection{Neimark-Sacker curves}

A nontrivial equilibrium to the amplitude system

$$
\begin{gathered}
\left(\rho_{1}, \rho_{2}\right)=\left(\sqrt{\frac{\beta_{2} \operatorname{Re} g_{1011}(0)-\beta_{1} \operatorname{Re} g_{0021}(0)}{\operatorname{Re} g_{0021}(0) \operatorname{Re} g_{2100}(0)-\operatorname{Re} g_{1011}(0) \operatorname{Re} g_{1110}(0)}},\right. \\
\left.\sqrt{\frac{\beta_{2} \operatorname{Re} g_{2100}(0)-\beta_{1} \operatorname{Re} g_{1110}(0)}{\operatorname{Re} g_{1011}(0) \operatorname{Re} g_{1110}(0)-\operatorname{Re} g_{0021}(0) \operatorname{Re} g_{2100}(0)}}\right)
\end{gathered}
$$

corresponds to a torus of the original system. When

$$
\operatorname{Re}\left(g_{1110}(0)\right) \beta_{1}=\operatorname{Re}\left(g_{2100}(0)\right) \beta_{2}
$$

the nontrivial equilibrium coincides with the first semi-trivial equilibrium, thus giving a predictor for a Neimark-Sacker bifurcation curve. Similarly, when

$$
\operatorname{Re}\left(g_{0021}(0)\right) \beta_{1}=\operatorname{Re}\left(g_{1011}(0)\right) \beta_{2}
$$

the nontrivial equilibrium coincides with the second semi-trivial equilibrium, and gives a predictor for the second Neimark-Sacker bifurcation curve. Therefore, we obtain two Neimark-Sacker bifurcation curves in (72), with approximations given by

$$
\begin{aligned}
& \left(\rho_{1}, \rho_{2}, \beta_{1}, \beta_{2}\right)=\left(\epsilon, 0,-\operatorname{Re}\left(g_{2100}(0)\right) \epsilon^{2},-\operatorname{Re}\left(g_{1110}(0)\right) \epsilon^{2}\right), \\
& \left(\rho_{1}, \rho_{2}, \beta_{1}, \beta_{2}\right)=\left(0, \epsilon,-\operatorname{Re}\left(g_{1011}(0)\right) \epsilon^{2},-\operatorname{Re}\left(g_{0021}(0)\right) \epsilon^{2}\right),
\end{aligned}
$$

where $\epsilon>0$, which are the predictors given in [46] and [49]. An approximation for the period of the cycle for each Neimark-Sacker predictor can be obtained from the third and fourth equation in system (73), yielding

$$
\left\{\begin{array}{l}
T_{1}=2 \pi /\left(\omega_{1}+b_{11} \beta_{1}+b_{12} \beta_{2}+\operatorname{Im}\left(g_{2100}(0)\right) \epsilon^{2}\right) \\
T_{2}=2 \pi /\left(\omega_{2}+b_{21} \beta_{1}+b_{22} \beta_{2}+\operatorname{Im}\left(g_{0021}(0)\right) \epsilon^{2}\right)
\end{array}\right.
$$

where

$$
b_{j k}=\frac{\partial b_{j}}{\partial \beta_{k}}(0), \quad j, k=1,2 .
$$

Here we should use $\left(\beta_{1}, \beta_{2}\right)$ as in (74a) and (74b).

\subsection{Transcritical-Hopf bifurcation}

A majority of papers in which fold-Hopf bifurcations in DDEs are studied, deals with models where the steady state remains fixed under variation of parameters. In this case the unfolding is not given by (67) anymore and we have to consider the smooth local normal form

$$
\left\{\begin{array}{c}
\dot{z}_{0}=\gamma(\alpha) z_{0}+g_{200}(\alpha) z_{0}^{2}+g_{011}(\alpha)\left|z_{1}\right|^{2}+g_{300}(\alpha) z_{0}^{3}+g_{111}(\alpha) z_{0}\left|z_{1}\right|^{2} \\
\quad+\mathcal{O}\left(\left\|\left(z_{0}, z_{1}, \bar{z}_{1}\right)\right\|^{4}\right), \\
\dot{z}_{1}=\lambda(\alpha) z_{1}+g_{110}(\alpha) z_{0} z_{1}+g_{210}(\alpha) w^{2} z_{1}+g_{021}(\alpha) z_{1}\left|z_{1}\right|^{2}+\mathcal{O}\left(\left\|\left(z_{0}, z_{1}, \bar{z}_{1}\right)\right\|^{4}\right) .
\end{array}\right.
$$

The bifurcation analysis can be carried out similar to the fold-Hopf case, see [29] and [40]. An alternative approach is presented in [62]. In contrast with the fold-Hopf bifurcation, there are in general two Neimark-Sacker bifurcation curves. Furthermore, the fold bifurcation curve becomes a transcritical bifurcation curve, and meets the Hopf bifurcation curve transversally. 
Under the assumption of the same transversality condition as in the fold-Hopf bifurcation we introduce new parameters $\left(\beta_{1}(\alpha), \beta_{2}(\alpha)\right)$ to obtain the truncated normal form

$$
\left\{\begin{array}{l}
\dot{z}_{0}=\beta_{1} z_{0}+g_{200}(\beta) z_{0}^{2}+g_{011}(\beta)\left|z_{1}\right|^{2}+g_{111}(\beta) z_{0}\left|z_{1}\right|^{2}+g_{300}(\beta) z_{0}^{3}, \\
\dot{z}_{1}=\left(\beta_{2}+i \omega_{0}+i b_{1}(\beta)\right) z_{1}+g_{110}(\beta) z_{0} z_{1}+g_{210}(\beta) z_{0}^{2} z+g_{021}(\beta) z_{1}\left|z_{1}\right|^{2},
\end{array}\right.
$$

with $b_{1}(0)=0$. Letting $z_{1}=\rho e^{i \psi}$ and separating the real and imaginary parts yields the three dimensional system

$$
\left\{\begin{aligned}
\dot{z}_{0} & =\beta_{1} z_{0}+g_{200}(\beta) z_{0}^{2}+g_{011}(\beta) \rho^{2}+g_{111}(\beta) z_{0} \rho^{2}+g_{300}(\beta) z_{0}^{3}, \\
\dot{\rho} & =\rho\left(\beta_{2}+\operatorname{Re}\left(g_{110}(\beta)\right) z_{0}+\operatorname{Re}\left(g_{210}(\beta)\right) z_{0}^{2}+\operatorname{Re}\left(g_{021}(\beta)\right) \rho^{2}\right), \\
\dot{\psi} & =\omega_{0}+b_{1}(\beta)+\operatorname{Im}\left(g_{110}(\beta)\right) z_{0}+\operatorname{Im}\left(g_{210}(\beta)\right) z_{0}^{2}+\operatorname{Im}\left(g_{021}(\beta)\right) \rho^{2} .
\end{aligned}\right.
$$

\subsubsection{Neimark-Sacker bifurcation curves}

Following the same procedure as in Section 5.2, we obtain that for $g_{011}(0) \operatorname{Re}\left(g_{110}(0)\right)<0$ there are two Neimark-Sacker bifurcation curves approximated by

$$
\left\{\begin{array}{l}
\beta_{1}=\mp 2 \sqrt{g_{011}(0) g_{200}(0)} \epsilon, \\
\beta_{2}=\mp \operatorname{Re}\left(g_{110}(0)\right) \sqrt{\frac{g_{011}(0)}{g_{200}(0)}} \epsilon, \\
z_{0}= \pm \sqrt{\frac{g_{011}(0)}{g_{200}(0)}} \epsilon,
\end{array}\right.
$$

while the period of the corresponding cycle is approximated by

$$
T=2 \pi /\left(\omega_{0}+\omega_{1} \beta_{1}+\omega_{2} \beta_{2}+\operatorname{Im}\left(g_{110}(0)\right) z_{0}\right) .
$$

Here $\left(z_{0}, \beta_{1}, \beta_{2}\right)$ are as in $(77)$ and $\omega_{i}=\frac{\partial b_{1}}{\partial \beta_{i}}(0)$, for $i=1,2$.

\subsubsection{Hopf and transcritical bifurcations curves}

The transcritical bifurcation curve in the normal form is obtained by substituting $\rho=0$ in the amplitude system of (76). Then $\beta_{2}$ is unrestricted and $z_{0}=-\beta_{1} / g_{200}(0)$. The transcritical bifurcation curve is therefore given by

$$
\left(\beta_{1}, \beta_{2}\right)=\left(0, \beta_{2}\right) .
$$

To obtain a predictor for the Hopf bifurcation curve we truncate (76) to the second order. We obtain a trivial equilibrium $\left(z_{0}, \rho\right)=(0,0)$, a semi-trivial equilibrium $\left(z_{0}, \rho\right)=\left(-\frac{\beta_{1}}{g_{200}(0)}, 0\right)$ and a nontrivial equilibrium

$$
\left(z_{0}, \rho\right)=\left(-\frac{\beta_{2}}{\operatorname{Re}\left(g_{110}(0)\right)}, \frac{\sqrt{\beta_{2}\left(\operatorname{Re}\left(g_{110}(0)\right) \beta_{1}-g_{200}(0) \beta_{2}\right)}}{\operatorname{Re}\left(g_{110}(0)\right) \sqrt{g_{011}(0)}}\right) .
$$

It follows that the Hopf bifurcation curves are approximated by

$$
\beta_{2}=\frac{\operatorname{Re}\left(g_{110}(0)\right)}{g_{200}(0)} \beta_{1}, \quad \beta_{2}=0 .
$$




\section{Coefficients of parameter-dependent normal forms}

Using the method outlined in Section 4, we derive here the coefficients needed for the predictors of the nonhyperbolic equilibria and cycles emanating from generalized Hopf, fold-Hopf, Hopf-Hopf and transcritical-Hopf bifurcations, see Section 5. While doing so, we also obtain the critical normal form coefficients, which were first derived in [37]. Recall, that we conside only two-parameter DDEs, so that $p=2$.

For the derivation of the coefficients in this section it is sufficient to expand and truncate the nonlinearity $R$ and the parameter-mapping $K$ in (57) and (58), respectively, as follows

$$
\begin{gathered}
R(u, \alpha)=\left(\frac{1}{2} B(u, u)+A_{1}(u, \alpha)+\frac{1}{2} J_{2}(\alpha, \alpha)+\frac{1}{6} C(u, u, u)+\frac{1}{2} B_{1}(u, u, \alpha)\right. \\
\left.+\frac{1}{24} D(u, u, u, u)+\frac{1}{6} C_{1}(u, u, u, \alpha)+\frac{1}{120} E(u, u, u, u, u)\right) r^{\odot \star} \\
\alpha=K(\beta)=K_{10} \beta_{1}+K_{01} \beta_{2} .
\end{gathered}
$$

Here $u \in X$, while $\alpha, \beta \in \mathbb{R}^{2}$, and $B, A_{1}, J_{2}, C, B_{1}, D, C_{1}$ and $E$ are the standard multilinear forms arising from the expansion of $F(u, \alpha)$ (or $G(u, \alpha)$ ). For example,

$$
B(u, u)=D_{1}^{2} F(0,0)(u, u), A_{1}(\alpha, \alpha)=D_{2}^{2} F(0,0)(\alpha, \alpha), B_{1}(u, u, \alpha)=D_{2}^{1} D_{1}^{2} F(0,0)(u, u, \alpha),
$$

etc. These forms are $\mathbb{R}^{n}$-valued on real arguments, while they are linearly extended ('complexified') to $\mathbb{C}^{n}$-valued ones on complex-valued arguments. Finally, we introduce

$$
J_{1}=D_{2} F(0,0) .
$$

We assume in all situations that $\varphi_{0}=0$ is a steady state of (28) at the critical parameter value $\alpha_{0}=0 \in \mathbb{R}^{2}$. Explicit formulas to compute the multilinear forms for the simplest DDE (1) are given in Section 7.

\subsection{Generalized Hopf bifurcation}

Since the eigenvalues (59) are simple, there exist eigenfunctions $\varphi$ and $\varphi^{\odot}$ such that

$$
A \varphi=i \omega_{0} \varphi, \quad A^{\star} \varphi^{\odot}=i \omega_{0} \varphi^{\odot}, \quad\left\langle\varphi^{\odot}, \varphi\right\rangle=1 .
$$

The eigenfunctions $\varphi$ and $\varphi^{\odot}$ are explicitly given by (22) and (23) with $q \in \mathbb{C}^{n}$ and $p \in \mathbb{C}^{n \star}$ satisfying

$$
\Delta\left(i \omega_{0}\right) q=0, \quad p \Delta\left(i \omega_{0}\right)=0, \quad p \Delta^{\prime}\left(i \omega_{0}\right) q=1 .
$$

Any point $y \in X_{0}$ in the real critical eigenspace can be represented as

$$
y=z \varphi+\bar{z} \bar{\varphi}, \quad z \in \mathbb{C},
$$

where $z=\left\langle\varphi^{\odot}, y\right\rangle$. Therefore, the homological equation (HOM) can be written as

$$
A^{\odot \star} j \mathcal{H}(z, \bar{z}, \beta)+J_{1} K(\beta) r^{\odot \star}+R(\mathcal{H}(z, \bar{z}, \beta), K(\beta))=j\left(D_{z} \mathcal{H}(z, \bar{z}, \beta) \dot{z}+D_{\bar{z}} \mathcal{H}(z, \bar{z}, \beta) \dot{\bar{z}}\right),
$$

where $\beta=\left(\beta_{1}, \beta_{2}\right), \dot{z}$ is given by the normal form (61) and $\mathcal{H}$ admits the expansion

$$
\mathcal{H}(z, \bar{z}, \beta)=z \varphi+\bar{z} \bar{\varphi}+H_{0010} \beta_{1}+H_{0001} \beta_{2}+\sum_{j+k+|\mu| \geq 2} \frac{1}{j ! k ! \mu !} H_{j k \mu} z^{j} \bar{z}^{k} \beta^{\mu} .
$$

For the predictors derived in Section 5.1 we need the truncated parameter-dependent normal form

$$
\dot{z}=\left(i \omega_{0}+\beta_{1}+i \omega_{10} \beta_{1}+i \omega_{01} \beta_{2}\right) z+\left(\beta_{2}+c_{1}(0)\right) z|z|^{2}+c_{2}(0) z|z|^{4},
$$

where $\operatorname{Re}\left(c_{1}(0)\right)=0$. 


\subsubsection{Critical normal form coefficients}

We start by calculating the critical normal form coefficients following [37]. Collecting the coefficients of the quadratic terms $z^{2}$ and $z \bar{z}$ in the homological equation yields two nonsingular linear systems:

$$
\begin{aligned}
\left(2 i \omega_{0}-A^{\odot \star}\right) j H_{2000} & =B(\varphi, \varphi) r^{\odot \star} \\
-A^{\odot \star} j H_{1100} & =B(\varphi, \bar{\varphi}) r^{\odot \star}
\end{aligned}
$$

They are solved using Lemma 3 to give

$$
\begin{aligned}
& H_{2000}(\theta)=e^{2 i \omega_{0} \theta} \Delta^{-1}\left(2 i \omega_{0}\right) B(\varphi, \varphi), \\
& H_{1100}(\theta)=\Delta^{-1}(0) B(\varphi, \bar{\varphi}) .
\end{aligned}
$$

For the cubic terms, the system corresponding to $z^{3}$ is also nonsingular,

$$
\left(3 i \omega_{0}-A^{\odot \star}\right) j H_{3000}=\left[3 B\left(\varphi, H_{2000}\right)+C(\varphi, \varphi, \varphi)\right] r^{\odot \star},
$$

with solution

$$
H_{3000}(\theta)=e^{3 i \omega_{0} \theta} \Delta^{-1}\left(3 i \omega_{0}\right)\left(3 B\left(\varphi, H_{2000}\right)+C(\varphi, \varphi, \varphi)\right) .
$$

On the other hand, the system corresponding to $z^{2} \bar{z}$ is singular,

$$
\left(i \omega_{0}-A^{\odot \star}\right) j H_{2100}=\left[B\left(\bar{\varphi}, H_{2000}\right)+2 B\left(\varphi, H_{1100}\right)+C(\varphi, \varphi, \bar{\varphi})\right] r^{\odot \star}-2 c_{1}(0) j \varphi .
$$

The Fredholm solvability condition (FSC) requires that

$$
c_{1}(0)=\frac{1}{2} p \cdot\left(B\left(\bar{\varphi}, H_{2000}\right)+2 B\left(\varphi, H_{1100}\right)+C(\varphi, \varphi, \bar{\varphi})\right),
$$

and from Lemma 4 we then obtain the unique solution satisfying $\left\langle\varphi^{\odot}, H_{2100}\right\rangle=0$ as

$$
H_{2100}(\theta)=B_{i \omega_{0}}^{\mathrm{INV}}\left(B\left(\bar{\varphi}, H_{2000}\right)+2 B\left(\varphi, H_{1100}\right)+C(\varphi, \varphi, \bar{\varphi}),-2 c_{1}(0)\right)(\theta) .
$$

We continue by collecting the coefficients corresponding to the fourth-order terms $z^{3} \bar{z}$ and $z^{2} \bar{z}^{2}$ in the homological equation. The corresponding nonsingular systems may be solved using Lemma 3 and the fact that $\operatorname{Re}\left(c_{1}(0)\right)=0$. For $H_{2200}$ this easily gives

$$
\begin{aligned}
& H_{2200}(\theta)=\Delta^{-1}(0)\left[2 B\left(\bar{\varphi}, H_{2100}\right)+2 B\left(\varphi, \bar{H}_{2100}\right)+B\left(\bar{H}_{2000}, H_{2000}\right)\right. \\
&+ 2 B\left(H_{1100}, H_{1100}\right)+C\left(\varphi, \varphi, \bar{H}_{2000}\right)+4 C\left(\varphi, \bar{\varphi}, H_{1100}\right) \\
&\left.+C\left(\bar{\varphi}, \bar{\varphi}, H_{2000}\right)+D(\varphi, \varphi, \bar{\varphi}, \bar{\varphi})\right],
\end{aligned}
$$

but for $H_{3100}$ the solution is a bit more subtle. The linear system is

$$
\begin{gathered}
\left(2 i \omega_{0}-A^{\odot \star}\right) j H_{3100}=[ \\
B\left(\bar{\varphi}, H_{3000}\right)+3 B\left(\varphi, H_{2100}\right)+3 B\left(H_{1100}, H_{2000}\right)+3 C\left(\varphi, \bar{\varphi}, H_{2000}\right) \\
\left.+3 C\left(\varphi, \varphi, H_{1100}\right)+D(\varphi, \varphi, \varphi, \bar{\varphi})\right] r^{\odot \star}-6 c_{1}(0) j H_{2000},
\end{gathered}
$$

so Lemma 3 applies with $w_{0}=[\cdots]-6 c_{1}(0) H_{2000}(0)$ and $w=-6 c_{1}(0) H_{2000}$ and we find

$$
\begin{aligned}
H_{3100}(\theta)= & e^{2 i \omega_{0} \theta} \Delta^{-1}\left(2 i \omega_{0}\right)\left[B\left(\bar{\varphi}, H_{3000}\right)+3 B\left(\varphi, H_{2100}\right)+3 B\left(H_{1100}, H_{2000}\right)\right. \\
& \left.+3 C\left(\varphi, \bar{\varphi}, H_{2000}\right)+3 C\left(\varphi, \varphi, H_{1100}\right)+D(\varphi, \varphi, \varphi, \bar{\varphi})\right] \\
& -6 c_{1}(0) \Delta^{-1}\left(2 i \omega_{0}\right)\left[\Delta^{\prime}\left(2 i \omega_{0}\right)-\theta \Delta\left(2 i \omega_{0}\right)\right] H_{2000}(\theta) .
\end{aligned}
$$


The critical normal form coefficient $c_{2}(0)$ is calculated by applying (FSC) to the singular linear system corresponding to the fifth-order term $z^{3} \bar{z}^{2}$ in the homological equation. This gives

$$
\begin{aligned}
c_{2}(0)=\frac{1}{12} & p \cdot\left[2 B\left(\bar{\varphi}, H_{3100}\right)+3 B\left(\varphi, H_{2200}\right)+B\left(\bar{H}_{2000}, H_{3000}\right)\right. \\
& +6 B\left(H_{1100}, H_{2100}\right)+3 B\left(\bar{H}_{2100}, H_{2000}\right) \\
& +6 C\left(\bar{\varphi}, H_{1100}, H_{2000}\right)+6 C\left(\varphi, \bar{\varphi}, H_{2100}\right)+C\left(\bar{\varphi}, \bar{\varphi}, H_{3000}\right) \\
& +3 C\left(\varphi, \varphi, \bar{H}_{2100}\right)+3 C\left(\varphi, \bar{H}_{2000}, H_{2000}\right)+6 C\left(\varphi, H_{1100}, H_{1100}\right) \\
& +D\left(\varphi, \varphi, \varphi, \bar{H}_{2000}\right)+6 D\left(\varphi, \varphi, \bar{\varphi}, H_{1100}\right)+3 D\left(\varphi, \bar{\varphi}, \bar{\varphi}, H_{2000}\right) \\
& +E(\varphi, \varphi, \varphi, \bar{\varphi}, \bar{\varphi})] .
\end{aligned}
$$

The second Lyapunov coefficient is now given by $\ell_{2}(0)=\frac{1}{\omega_{0}} \operatorname{Re}\left(c_{2}(0)\right)$.

\subsubsection{Parameter-related coefficients}

Next we derive the parameter-related coefficients that provide a linear approximation to the parameter transformation. Following [46] and [2] we first expand the eigenvalue $\lambda(\alpha)$ and $c_{1}(\alpha)$ in the normal form (60) in the original parameters $\alpha$ and truncate to fourth order,

$$
\dot{z}=\left(i \omega_{0}+\gamma_{110} \alpha_{1}+\gamma_{101} \alpha_{2}\right) z+\left(c_{1}(0)+\gamma_{210} \alpha_{1}+\gamma_{201} \alpha_{2}\right) z|z|^{2}+c_{2}(0) z|z|^{4} .
$$

The parameters $\alpha$ and $\beta$ are related via

$$
\alpha=\left(\operatorname{Re}\left(\begin{array}{ll}
\gamma_{110} & \gamma_{101} \\
\gamma_{210} & \gamma_{201}
\end{array}\right)\right)^{-1} \beta
$$

so that

$$
\frac{\partial b_{1}}{\partial \beta_{2}}(0)=\operatorname{Im}\left(\left(\begin{array}{ll}
\gamma_{110} & \gamma_{101}
\end{array}\right)\left(\operatorname{Re}\left(\begin{array}{ll}
\gamma_{110} & \gamma_{101} \\
\gamma_{210} & \gamma_{201}
\end{array}\right)\right)^{-1}\left(\begin{array}{l}
0 \\
1
\end{array}\right)\right) .
$$

The homological equation (HOM) becomes

$$
A^{\odot \star} j \mathcal{H}(z, \bar{z}, \alpha)+J_{1} \alpha r^{\odot \star}+R(\mathcal{H}(z, \bar{z}, \alpha), \alpha)=j\left(D_{z} \mathcal{H}(z, \bar{z}, \alpha) \dot{z}+D_{\bar{z}} \mathcal{H}(z, \bar{z}, \alpha) \dot{\bar{z}}\right),
$$

where $\mathcal{H}$ admits the expansion

$$
u=\mathcal{H}\left(z, \bar{z}, \alpha_{1}, \alpha_{2}\right)=z \varphi+\bar{z} \bar{\varphi}+H_{0010} \alpha_{1}+H_{0001} \alpha_{2}+\sum_{j+k+|\mu| \geq 2} \frac{1}{j ! k ! \mu !} H_{j k \mu} z^{j} \bar{z}^{k} \alpha^{\mu}
$$

and $R$ is given by (78). Notice that the coefficients $H_{j k \mu}$ with $\mu=(00)$ in (84) coincide with those in the expansion (81). Collecting the coefficients of the terms $\alpha$ and $z \alpha$ in the homological equation yields the systems

$$
\begin{aligned}
-A^{\odot \star} j H_{00 \mu} & =J_{1} v_{\mu} r^{\odot \star} \\
\left(i \omega_{0}-A^{\odot \star}\right) j H_{10 \mu} & =\left[A_{1}\left(\varphi, v_{\mu}\right)+B\left(\varphi, H_{00 \mu}\right)\right] r^{\odot \star}-\gamma_{1 \mu} j \varphi
\end{aligned}
$$

where $\mu=(10),(01)$ and $v_{10}=(1,0)^{T}, v_{01}=(0,1)^{T}$. We solve these systems using Section 2.6. By the first part of Lemma 3 the first system has the (constant) solutions

$$
H_{00 \mu}(\theta)=\Delta^{-1}(0) J_{1} v_{\mu}
$$

and (FSC) gives

$$
\gamma_{1 \mu}=p\left(A_{1}\left(\varphi, v_{\mu}\right)+B\left(\varphi, H_{00 \mu}\right)\right) .
$$


Using Lemma 4 we obtain the solutions

$$
H_{10 \mu}(\theta)=B_{i \omega_{0}}^{\mathrm{INV}}\left(A_{1}\left(\varphi, v_{\mu}\right)+B\left(\varphi, H_{00 \mu}\right),-\gamma_{1 \mu}\right)(\theta)
$$

for the second equation. To determine $\gamma_{2 \mu}$ we first collect the coefficients corresponding to the $z^{2} \alpha$ and $z \bar{z} \alpha$ terms in the homological equation. We obtain the equations

$$
\begin{aligned}
\left(2 i \omega_{0}-A^{\odot \star}\right) j H_{20 \mu}=[ & A_{1}\left(H_{2000}, v_{\mu}\right)+2 B\left(\varphi, H_{10 \mu}\right)+B\left(H_{2000}, H_{00 \mu}\right)+B_{1}\left(\varphi, \varphi, v_{\mu}\right) \\
& \left.+C\left(\varphi, \varphi, H_{00 \mu}\right)\right] r^{\odot \star}-2 \gamma_{1 \mu} j H_{2000}, \\
-A^{\odot \star} j H_{11 \mu}=[ & A_{1}\left(H_{1100}, v_{\mu}\right)+2 \operatorname{Re}\left(B\left(\bar{\varphi}, H_{10 \mu}\right)\right)+B\left(H_{1100}, H_{00 \mu}\right)+B_{1}\left(\varphi, \bar{\varphi}, v_{\mu}\right) \\
+ & \left.+\left(\varphi, \bar{\varphi}, H_{0 \mu}\right)\right] r^{\odot \star}-2 \operatorname{Re}\left(\gamma_{1 \mu}\right) j H_{1100} .
\end{aligned}
$$

Lemma 3 implies that solutions of the first two equations are given by

$$
\begin{gathered}
H_{20 \mu}(\theta)=e^{2 i \omega_{0} \theta} \Delta^{-1}\left(2 i \omega_{0}\right)\left[A_{1}\left(H_{2000}, v_{\mu}\right)+2 B\left(\varphi, H_{10 \mu}\right)+B\left(H_{2000}, H_{00 \mu}\right)+B_{1}\left(\varphi, \varphi, v_{\mu}\right)\right. \\
\left.+C\left(\varphi, \varphi, H_{00 \mu}\right)\right]-2 \gamma_{1 \mu} \Delta\left(2 i \omega_{0}\right)^{-1}\left(\Delta^{\prime}(2 i \omega)-\theta \Delta\left(2 i \omega_{0}\right)\right) H_{2000}(\theta), \\
H_{11 \mu}(\theta)=\Delta^{-1}(0)\left[A_{1}\left(H_{1100}, v_{\mu}\right)+2 \operatorname{Re}\left(B\left(\bar{\varphi}, H_{10 \mu}\right)\right)+B\left(H_{1100}, H_{00 \mu}\right)+B_{1}\left(\varphi, \bar{\varphi}, v_{\mu}\right)\right. \\
\left.+C\left(\varphi, \bar{\varphi}, H_{0 \mu}\right)\right]-2 \operatorname{Re}\left(\gamma_{1 \mu}\right) \Delta(0)^{-1}\left(\Delta^{\prime}(0)-\theta \Delta(0)\right) H_{1100}(\theta) .
\end{gathered}
$$

Applying (FSC) to $z^{2} \bar{z} \alpha$ terms in the homological equation results in

$$
\begin{aligned}
\gamma_{2 \mu}=\frac{1}{2} & p \cdot\left[A_{1}\left(H_{2100}, v_{\mu}\right)+B\left(\bar{\varphi}, H_{20 \mu}\right)+2 B\left(\varphi, H_{11 \mu}\right)\right. \\
& +B\left(H_{2100}, H_{00 \mu}\right)+B\left(H_{2000}, \bar{H}_{10 \mu}\right)+2 B\left(H_{1100}, H_{10 \mu}\right) \\
& +B_{1}\left(H_{2000}, \bar{\varphi}, v_{\mu}\right)+2 B_{1}\left(\varphi, H_{1100}, v_{\mu}\right)+2 C\left(\varphi, \bar{\varphi}, H_{10 \mu}\right) \\
& +C\left(H_{2000}, \bar{\varphi}, H_{00 \mu}\right)+C\left(\varphi, \varphi, H_{01 \mu}\right)+2 C\left(\varphi, H_{1100}, H_{00 \mu}\right) \\
& \left.+C_{1}\left(\varphi, \varphi, \bar{\varphi}, v_{\mu}\right)+D\left(\varphi, \varphi, \bar{\varphi}, H_{00 \mu}\right)\right] .
\end{aligned}
$$

\subsubsection{Hopf and LPC predictors}

Now we are ready to specify the predictors for the original parameter-dependent DDE (28). To approximate the Hopf parameter values $\alpha$ and the corresponding equilibrium, we merely substitute $\beta$ from (65) into (82), and then put the result together with $z=0$ into the expansion (84).

To approximate the LPC parameter values, we substitute $\beta$ from (63) into (82). The cycle period is approximated by (64) with (83). To obtain a predictor for the periodic orbit in the phase space, we set $z=\epsilon e^{i \psi}$ into (84) using the obtained $\alpha$ values. Truncating to the second order in $\epsilon$ then yields

$$
u=2 \operatorname{Re}\left(e^{i \psi} \varphi\right) \epsilon+\left(H_{1100}-2 \operatorname{Re}\left(c_{2}(0)\right) H_{0001}+\operatorname{Re}\left(e^{2 i \psi} H_{2000}\right)\right) \epsilon^{2}, \quad \psi \in[0,2 \pi] .
$$

\subsection{Fold-Hopf bifurcation}

Since the eigenvalues (66) are simple, there exist eigenfunctions $\varphi_{0,1}$ and $\varphi_{0,1}^{\odot}$ satisfying

$$
A \varphi_{0}=0, \quad A \varphi_{1}=i \omega_{0} \varphi_{1}, \quad A^{\star} \varphi_{0}^{\odot}=0, \quad A^{\star} \varphi_{1}^{\odot}=i \omega_{0} \varphi_{1}^{\odot}
$$

as well as the mutual normalization condition

$$
\left\langle\varphi_{i}^{\odot}, \varphi_{j}\right\rangle=\delta_{i j}, \quad 0 \leq i, j \leq 1 .
$$

The eigenfunctions $\varphi_{0,1}$ and $\varphi_{0,1}^{\odot}$ can be explicitly computed using (22) and (23) with $q_{0} \in \mathbb{R}^{n}, q_{1} \in \mathbb{C}^{n}$, $p_{0} \in \mathbb{R}^{n \star}$ and $p_{1} \in \mathbb{C}^{n \star}$ satisfying

$$
\Delta(0) q_{0}=0, \quad \Delta\left(i \omega_{0}\right) q_{1}=0, \quad p_{0} \Delta(0)=0, \quad p_{1} \Delta\left(i \omega_{0}\right)=0,
$$


as well as

$$
p_{0} \Delta^{\prime}(0) q_{0}=1, \quad p_{1} \Delta^{\prime}\left(i \omega_{0}\right) q_{1}=1
$$

Any point $y \in X_{0}$ in the real critical eigenspace can be represented as

$$
y=z_{0} \varphi_{0}+z_{1} \varphi_{1}+\bar{z}_{1} \bar{\varphi}_{1}, \quad\left(z_{0}, z_{1}\right) \in \mathbb{R} \times \mathbb{C},
$$

where $z_{0}=\left\langle\varphi_{0}^{\odot}, y\right\rangle$ and $z_{1}=\left\langle\varphi_{1}^{\odot}, y\right\rangle$. Therefore, the homological equation (HOM) can be written as

$$
\begin{aligned}
& A^{\odot \star} j \mathcal{H}(z, \beta)+J_{1}(\beta) r^{\odot \star}+R(\mathcal{H}(z, \beta), K(\beta)) \\
& \quad=j\left(D_{z_{0}} \mathcal{H}(z, \beta) \dot{z}_{0}+D_{z_{1}} \mathcal{H}(z, \beta) \dot{z}_{1}+D_{\bar{z}_{1}} \mathcal{H}(z, \beta) \dot{\bar{z}}_{1}\right),
\end{aligned}
$$

where $z=\left(z_{0}, z_{1}, \bar{z}_{1}\right), \beta=\left(\beta_{1}, \beta_{2}\right)$ and $\dot{z}$ is given by the normal form (68). Here, the mapping $\mathcal{H}$ admits the expansion

$$
\begin{aligned}
\mathcal{H}\left(z_{0}, z_{1}, \bar{z}_{1}, \beta\right) & =z_{0} \varphi_{0}+z_{1} \varphi_{1}+\bar{z}_{1} \bar{\varphi}_{1}+H_{00010} \beta_{1}+H_{00001} \beta_{2} \\
& +\sum_{j+k+l+|\mu| \geq 2} \frac{1}{j ! k ! l ! \mu !} H_{j k l \mu} z_{0}^{j} z_{1}^{k} \bar{z}_{1}^{l} \beta^{\mu}
\end{aligned}
$$

and the functions $K$ and $R$ are as in (78) and (79), respectively.

\subsubsection{Critical normal form coefficients}

We start by computing the critical normal form coefficients following [37]. Collecting the quadratic terms $z_{0}^{2}, z_{1}^{2}, z_{0} z_{1}$ and $z_{1} \bar{z}_{1}$ we obtain one nonsingular and three singular linear systems. By (FSC) the singular systems are consistent if and only if

$$
g_{200}(0)=\frac{1}{2} p_{0} B\left(\varphi_{0}, \varphi_{0}\right), \quad g_{110}(0)=p_{1} B\left(\varphi_{0}, \varphi_{1}\right), \quad g_{011}(0)=p_{0} B\left(\varphi_{1}, \bar{\varphi}_{1}\right) .
$$

This yields the three quadratic normal form coefficients. The corresponding solutions may then be obtained using Lemmas 3 and 4. Namely,

$$
\begin{aligned}
& H_{20000}(\theta)=B_{0}^{\mathrm{INV}}\left(B\left(\varphi_{0}, \varphi_{0}\right),-2 g_{200}(0)\right)(\theta), \\
& H_{02000}(\theta)=e^{2 i \omega_{0} \theta} \Delta^{-1}\left(2 i \omega_{0}\right) B\left(\varphi_{1}, \varphi_{1}\right), \\
& H_{11000}(\theta)=B_{i \omega_{0}}^{\mathrm{INV}}\left(B\left(\varphi_{0}, \varphi_{1}\right),-g_{110}(0)\right)(\theta), \\
& H_{01100}(\theta)=B_{0}^{\mathrm{INV}}\left(B\left(\varphi_{1}, \bar{\varphi}_{1}\right),-g_{011}(0)\right)(\theta) .
\end{aligned}
$$

For the four remaining cubic normal form coefficients, we collect the coefficients of the resonant terms $z_{0}^{j} z_{1}^{k} \bar{z}_{1}^{l}$ in (85) with $j+k+l=3$. This yields four singular linear systems. As before, by (FSC) these systems are consistent if and only if

$$
\begin{aligned}
& g_{300}(0)=\frac{1}{6} p_{0}\left(3 B\left(\varphi_{0}, H_{20000}\right)+C\left(\varphi_{0}, \varphi_{0}, \varphi_{0}\right)\right), \\
& g_{111}(0)=p_{0}\left(B\left(\varphi_{0}, H_{01100}\right)+B\left(\varphi_{1}, \bar{H}_{11000}\right)+B\left(\bar{\varphi}_{1}, H_{11000}\right)+C\left(\varphi_{0}, \varphi_{1}, \bar{\varphi}_{1}\right)\right), \\
& g_{210}(0)=\frac{1}{2} p_{1}\left(2 B\left(\varphi_{0}, H_{11000}\right)+B\left(\varphi_{1}, H_{20000}\right)+C\left(\varphi_{0}, \varphi_{0}, \varphi_{1}\right)\right), \\
& g_{021}(0)=\frac{1}{2} p_{1}\left(2 B\left(\varphi_{1}, H_{01100}\right)+B\left(\bar{\varphi}_{1}, H_{02000}\right)+C\left(\varphi_{1}, \varphi_{1}, \bar{\varphi}_{1}\right)\right) .
\end{aligned}
$$




\subsubsection{Parameter-related coefficients}

The parameter-related linear terms in (85) give

$$
\begin{aligned}
-A^{\odot \star} j H_{00010} & =J_{1} K_{10} r^{\odot \star}-j \varphi_{0}, \\
-A^{\odot \star} j H_{00001} & =J_{1} K_{01} r^{\odot \star}
\end{aligned}
$$

Let $\gamma=\left(\gamma_{1}, \gamma_{2}\right)=p_{0}^{T} J_{1}$. Then by (FSC) we obtain the orthogonal frame

$$
K_{10}=s_{1}+\delta_{1} s_{2}, \quad K_{01}=\delta_{2} s_{2},
$$

where

$$
s_{1}^{T}=\gamma /\|\gamma\|^{2}, \quad s_{2}^{T}=\left(-\gamma_{2}, \gamma_{1}\right)
$$

and $\delta_{1,2} \in \mathbb{R}$ are constants. Using Lemma 4 from Section 2.6 we get

$$
\begin{aligned}
H_{00010}(\theta) & =\Delta^{\mathrm{INV}}(0)\left(J_{1} K_{10}-\Delta^{\prime}(0) q_{0}\right)+\delta_{3} q_{0}+\theta q_{0} \\
& =r_{1}+\delta_{1} r_{2}+\delta_{3} q_{0}-r_{3}(\theta) \\
H_{00001}(\theta) & =\delta_{2} r_{2}+\delta_{4} q_{0}
\end{aligned}
$$

where

$$
r_{1}=\Delta^{\mathrm{INV}}(0)\left(J_{1} s_{1}\right), \quad r_{2}=\Delta^{\mathrm{INV}}(0)\left(J_{1} s_{2}\right), \quad r_{3}(\theta)=\Delta^{\mathrm{INV}}(0)\left(\Delta^{\prime}(0) q_{0}\right)-\theta q_{0},
$$

and the real constants $\delta_{3}$ and $\delta_{4}$ are not chosen such that $\left\langle\varphi_{0}^{\odot}, H_{00010}\right\rangle=0$ and $\left\langle\varphi_{0}^{\odot}, H_{00001}\right\rangle=0$, but will be determined below. Collecting the $z_{0} \beta$ and $z_{1} \beta$ terms in the homological equation yields the systems

$$
\begin{aligned}
-A^{\odot \star} j H_{10010} & =\left[B\left(\varphi_{0}, H_{00010}\right)+A_{1}\left(\varphi_{0}, K_{10}\right)\right] r^{\odot \star}-j H_{20000}, \\
-A^{\odot \star} j H_{10001} & =\left[B\left(\varphi_{0}, H_{00001}\right)+A_{1}\left(\varphi_{0}, K_{01}\right)\right] r^{\odot \star}, \\
\left(i \omega_{0}-A^{\odot \star}\right) j H_{01010} & =\left[B\left(\varphi_{1}, H_{00010}\right)+A_{1}\left(\varphi_{1}, K_{10}\right)\right] r^{\odot \star}-j\left(i \omega_{1} \varphi_{1}+H_{11000}\right), \\
\left(i \omega_{0}-A^{\odot \star}\right) j H_{01001} & =\left[B\left(\varphi_{1}, H_{00001}\right)+A_{1}\left(\varphi_{1}, K_{01}\right)\right] r^{\odot \star}-\left(1+i \omega_{2}\right) j \varphi_{1} .
\end{aligned}
$$

Notice that the coefficients $\omega_{1,2}$ were introduced in Section 5.2.1. To determine $\delta_{i}(i=1,2,3,4)$ we substitute (87) and (88) into (89). Then by (FSC) we obtain the system

$$
\begin{aligned}
\left(\begin{array}{cc}
p_{0} B\left(\varphi_{0}, r_{2}\right)+p_{0} A_{1}\left(\varphi_{0}, s_{2}\right) & 2 g_{200}(0) \\
\operatorname{Re}\left(p_{1} B\left(\varphi_{1}, r_{2}\right)+p_{1} A_{1}\left(\varphi_{1}, s_{2}\right)\right) & \operatorname{Re}\left(g_{110}(0)\right)
\end{array}\right)\left(\begin{array}{cc}
\delta_{1} & \delta_{2} \\
\delta_{3} & \delta_{4}
\end{array}\right)= \\
\quad\left(\begin{array}{ccc}
-p_{0}\left(A_{1}\left(\varphi_{0}, s_{1}\right)+B\left(\varphi_{0}, r_{1}-r_{3}\right)\right) & 0 \\
-\operatorname{Re}\left(p_{1}\left(A_{1}\left(\varphi_{1}, s_{1}\right)+B\left(\varphi_{1}, r_{1}-r_{3}\right)\right)\right) & 1
\end{array}\right)
\end{aligned}
$$

Subsequently, the coefficients $\omega_{1}$ and $\omega_{2}$ are given by

$$
\begin{aligned}
& \omega_{1}=\operatorname{Im}\left(p_{1} B\left(\varphi_{1}, H_{00010}\right)+p_{1} A_{1}\left(\varphi_{1}, K_{10}\right)\right), \\
& \omega_{2}=\operatorname{Im}\left(p_{1} B\left(\varphi_{1}, H_{00001}\right)+p_{1} A_{1}\left(\varphi_{1}, K_{01}\right)\right) .
\end{aligned}
$$

\subsubsection{Hopf, fold, and Neimark-Sacker predictors}

To approximate the fold and Hopf curves and their corresponding equilibria, one should substitute the expressions for $\beta$ and the equilibrium coordinates given in Section 5.2.2 into the expansions (86) and $(79)$. 
To approximate the periodic orbit at the Neimark-Sacker bifurcation, we substitute $z_{1}=\epsilon e^{i \psi}$ and (70) into (86). After a truncation this gives

$$
\begin{aligned}
u= & 2 \operatorname{Re}\left(e^{i \psi} \varphi_{1}\right) \epsilon+\left(\frac{\operatorname{Re}\left(g_{110}(0)\right)\left(2 \operatorname{Re}\left(g_{021}(0)\right)+g_{111}(0)\right)-2 \operatorname{Re}\left(g_{021}(0)\right) g_{200}(0)}{2 g_{200}(0)} H_{00001}\right. \\
& \left.-g_{011}(0) H_{00010}+H_{01100}-\left(\frac{2 \operatorname{Re}\left(g_{021}(0)\right)+g_{111}(0)}{2 g_{200}(0)}\right) \varphi_{0}+\operatorname{Re}\left(e^{2 i \psi} \bar{H}_{02000}\right)\right) \epsilon^{2},
\end{aligned}
$$

where $\psi \in[0,2 \pi]$.

\subsection{Hopf-Hopf bifurcation}

Since the eigenvalues (71) are simple, there exist eigenfunctions $\varphi_{1,2}$ and $\varphi_{1,2}^{\odot}$,

$$
A \varphi_{1}=i \omega_{1} \varphi_{1}, \quad A \varphi_{2}=i \omega_{2} \varphi_{2}, \quad A^{\star} \varphi_{1}^{\odot}=i \omega_{1} \varphi_{1}^{\odot}, \quad A^{\star} \varphi_{2}^{\odot}=i \omega_{2} \varphi_{2}^{\odot}
$$

satisfying the mutual normalization conditions

$$
\left\langle\varphi_{i}^{\odot}, \varphi_{j}\right\rangle=\delta_{i j}, \quad 1 \leq i, j \leq 2 .
$$

The eigenfunctions $\varphi_{1,2}$ and $\varphi_{1,2}^{\odot}$ can be explicitly computed using (22) and (23) with $q_{1,2} \in \mathbb{C}^{n}$ and $p_{1,2} \in \mathbb{C}^{n \star}$ such that both

$$
\Delta\left(i \omega_{1}\right) q_{1}=0, \quad \Delta\left(i \omega_{2}\right) q_{2}=0, \quad p_{1} \Delta\left(i \omega_{1}\right)=0, \quad p_{2} \Delta\left(i \omega_{2}\right)=0,
$$

as well as

$$
p_{1} \Delta^{\prime}\left(i \omega_{1}\right) q_{1}=1, \quad p_{2} \Delta^{\prime}\left(i \omega_{2}\right) q_{2}=1 .
$$

Any point $y \in X_{0}$ in the real critical eigenspace can be represented as

$$
y=z_{1} \varphi_{1}+\bar{z}_{1} \bar{\varphi}_{1}+z_{2} \varphi_{2}+\bar{z}_{2} \bar{\varphi}_{2}, \quad z_{1,2} \in \mathbb{C},
$$

where $z_{1}=\left\langle\varphi_{1}^{\odot}, y\right\rangle$ and $z_{2}=\left\langle\varphi_{2}^{\odot}, y\right\rangle$. Therefore, the homological equation (HOM) can be written as

$$
\begin{aligned}
& A^{\odot \star} \mathcal{H}(z, \beta)+J_{1}(\beta) r^{\odot \star}+R(\mathcal{H}(z, \beta), K(\beta)) \\
& \quad=j\left(D_{z_{1}} \mathcal{H}(z, \beta) \dot{z}_{1}+D_{\bar{z}_{1}} \mathcal{H}(z, \beta) \bar{z}_{1}+D_{z_{2}} \mathcal{H}(z, \beta) \dot{z}_{2}+D_{\bar{z}_{2}} \mathcal{H}(z, \beta) \dot{\bar{z}}_{2}\right),
\end{aligned}
$$

where $z=\left(z_{1}, \bar{z}_{1}, z_{2}, \bar{z}_{2}\right), \beta=\left(\beta_{1}, \beta_{2}\right)$ and $\dot{z}$ is given by the normal form (72). The mapping $\mathcal{H}$ admits the expansion

$$
\begin{aligned}
\mathcal{H}\left(z_{1}, \bar{z}_{1}, z_{2}, \bar{z}_{2}, \beta_{1}, \beta_{2}\right) & =z_{1} \varphi_{1}+\bar{z}_{1} \bar{\varphi}_{1}+z_{2} \varphi_{2}+\bar{z}_{2} \bar{\varphi}_{2}+H_{000010} \beta_{1}+H_{000001} \beta_{2} \\
& +\sum_{j+k+l+m+|\mu| \geq 2} \frac{1}{j ! k ! l ! m ! \mu !} H_{j k l m \mu} z_{1}^{j} \bar{z}_{1}^{k} z_{2}^{l} \bar{z}_{2}^{m} \beta^{\mu}
\end{aligned}
$$

and the functions $K$ and $R$ are as in (78) and (79), respectively.

\subsubsection{Critical normal form coefficients}

For initialization of the Neimark-Sacker curves (74) we need the cubic critical normal form coefficients $g_{2100}(0), g_{1011}(0), g_{1110}(0)$ and $g_{0021}(0)$. We compute these coefficients following [37]. 
Collecting the coefficients of the quadratic terms $\left|z_{1}\right|^{2}, z_{1}^{2}, z_{1} z_{2},\left|z_{2}\right|^{2}, z_{1} \bar{z}_{2}$ and $z_{2} \bar{z}_{1}$ in the homological equation, we obtain six nonsingular linear systems. By Lemma 3 their solutions are

$$
\begin{aligned}
& H_{110000}(\theta)=\Delta^{-1}(0) B\left(\varphi_{1}, \bar{\varphi}_{1}\right), \\
& H_{200000}(\theta)=e^{2 i \omega_{1} \theta} \Delta^{-1}\left(2 i \omega_{1}\right) B\left(\varphi_{1}, \varphi_{1}\right), \\
& H_{101000}(\theta)=e^{i\left(\omega_{1}+\omega_{2}\right) \theta} \Delta^{-1}\left(i\left(\omega_{1}+\omega_{2}\right)\right) B\left(\varphi_{1}, \varphi_{2}\right), \\
& H_{001100}(\theta)=\Delta^{-1}(0) B\left(\varphi_{2}, \bar{\varphi}_{2}\right), \\
& H_{100100}(\theta)=e^{i\left(\omega_{1}-\omega_{2}\right) \theta} \Delta^{-1}\left(i\left(\omega_{1}-\omega_{2}\right)\right) B\left(\varphi_{1}, \bar{\varphi}_{2}\right), \\
& H_{002000}(\theta)=e^{2 i \omega_{2} \theta} \Delta^{-1}\left(2 i \omega_{2}\right) B\left(\varphi_{2}, \varphi_{2}\right) .
\end{aligned}
$$

The desired cubic critical normal form coefficients are obtained by collecting the coefficients of the resonant cubic terms $z_{1}\left|z_{1}\right|^{2}, z_{1}\left|z_{2}\right|^{2},\left|z_{1}\right|^{2} z_{2}$ and $\left|z_{2}\right|^{2} z_{2}$ in the homological equation. This leads to four singular linear systems. By (FSC) these systems are solvable if and only if

$$
\begin{aligned}
& g_{2100}(0)=\frac{1}{2} p_{1}\left(2 B\left(\varphi_{1}, H_{110000}\right)+B\left(\bar{\varphi}_{1}, H_{200000}\right)+C\left(\varphi_{1}, \varphi_{1}, \bar{\varphi}_{1}\right)\right), \\
& g_{1011}(0)=p_{1}\left(B\left(\bar{\varphi}_{2}, H_{101000}\right)+B\left(\varphi_{1}, H_{001100}\right)+B\left(\varphi_{2}, H_{100100}\right)+C\left(\varphi_{1}, \varphi_{2}, \bar{\varphi}_{2}\right)\right), \\
& g_{1110}(0)=p_{2}\left(B\left(\bar{\varphi}_{1}, H_{101000}\right)+B\left(\varphi_{1}, \bar{H}_{100100}\right)+B\left(\varphi_{2}, H_{110000}\right)+C\left(\varphi_{1}, \bar{\varphi}_{1}, \varphi_{2}\right)\right), \\
& g_{0021}(0)=\frac{1}{2} p_{2}\left(2 B\left(\varphi_{2}, H_{001100}\right)+B\left(\bar{\varphi}_{2}, H_{002000}\right)+C\left(\varphi_{2}, \varphi_{2}, \bar{\varphi}_{2}\right)\right) .
\end{aligned}
$$

\subsubsection{Parameter-related coefficients}

The linear terms in (91) give back the eigenfunctions (90) and the parameter-related equations

$$
-A^{\odot \star} j H_{0000 \mu}=J_{1} K_{\mu} r^{\odot \star},
$$

where $\mu=(10),(01)$. Let

$$
K_{\mu}=\gamma_{1 \mu} e_{1}+\gamma_{2 \mu} e_{2},
$$

where $e_{1}=(1,0), e_{2}=(0,1)$ and $\gamma_{i \mu}(i=1,2) \in \mathbb{R}$ are constants to be determined. Then Lemma 3 from Section 2.6 implies

$$
H_{0000 \mu}(\theta)=\gamma_{1 \mu} \Delta^{-1}(0) J_{1} e_{1}+\gamma_{2 \mu} \Delta^{-1}(0) J_{1} e_{2} .
$$

Collecting the $z_{i} \beta_{j}$-terms with $1 \leq i, j \leq 2$ yields the systems

$$
\begin{aligned}
\left(i \omega_{1}-A^{\odot \star}\right) j H_{100010} & =\left[A_{1}\left(\varphi_{1}, K_{10}\right)+B\left(\varphi_{1}, H_{000010}\right)\right] r^{\odot \star}-\left(1+i b_{11}\right) j \varphi_{1}, \\
\left(i \omega_{1}-A^{\odot \star}\right) j H_{100001} & =\left[A_{1}\left(\varphi_{1}, K_{01}\right)+B\left(\varphi_{1}, H_{000001}\right)\right] r^{\odot \star}-i b_{12} j \varphi_{1}, \\
\left(i \omega_{2}-A^{\odot \star}\right) j H_{001010} & =\left[A_{1}\left(\varphi_{2}, K_{10}\right)+B\left(\varphi_{2}, H_{000010}\right)\right] r^{\odot \star}-i b_{21} j \varphi_{2}, \\
\left(i \omega_{2}-A^{\odot \star}\right) j H_{001001} & =\left[A_{1}\left(\varphi_{2}, K_{01}\right)+B\left(\varphi_{2}, H_{000001}\right)\right] r^{\odot \star}-\left(1+i b_{22}\right) j \varphi_{2},
\end{aligned}
$$

where $b_{j k}$ are defined in Section 5.3.2. To determine $\gamma_{i \mu}(i=1,2)$ we substitute (93) and (94) into (95). Then by (FSC) we obtain the system

$$
\operatorname{Re}\left[\left(\begin{array}{ll}
\Gamma_{11} & \Gamma_{12} \\
\Gamma_{31} & \Gamma_{32}
\end{array}\right)\right]\left(\begin{array}{ll}
\gamma_{110} & \gamma_{210} \\
\gamma_{101} & \gamma_{201}
\end{array}\right)=\left(\begin{array}{ll}
1 & 0 \\
0 & 1
\end{array}\right),
$$

where

$$
\Gamma_{i j}:=A_{1}\left(\varphi_{i}, e_{j}\right)+B\left(\varphi_{i}, \Delta^{-1}(0) J_{1} e_{j}\right), \quad 1 \leq i, j \leq 2 .
$$

Note that $\Delta^{-1}(0) J_{1} e_{i}$ is a constant function of $\theta$. 
It now follows from (95) that the coefficients $b_{11}, b_{12}, b_{21}$ and $b_{22}$, needed for the second order approximation of the periods, are given by

$$
\begin{aligned}
& b_{11}=\operatorname{Im}\left(p_{1}\left(A_{1}\left(\varphi_{1}, K_{10}\right)+B\left(\varphi_{1}, H_{000010}\right)\right)\right), \\
& b_{12}=\operatorname{Im}\left(p_{1}\left(A_{1}\left(\varphi_{1}, K_{01}\right)+B\left(\varphi_{1}, H_{000001}\right)\right)\right), \\
& b_{21}=\operatorname{Im}\left(p_{2}\left(A_{1}\left(\varphi_{2}, K_{10}\right)+B\left(\varphi_{2}, H_{000010}\right)\right)\right), \\
& b_{22}=\operatorname{Im}\left(p_{2}\left(A_{1}\left(\varphi_{2}, K_{01}\right)+B\left(\varphi_{2}, H_{000001}\right)\right)\right) .
\end{aligned}
$$

\subsubsection{Hopf and Neimark-Sacker predictors}

To approximate the Hopf curves and their corresponding equilibria, one should substitute the expressions for $\beta$ and the equilibrium coordinates given in Section 5.3.1 into the expansions (92) and (79).

To approximate the Neimark-Sacker periodic orbits, we substitute $\left(z_{1}, z_{2}\right)=\left(\epsilon e^{i \psi_{1}}, 0\right)$ and $(74 \mathrm{a})$, and $\left(z_{1}, z_{2}\right)=\left(0, \epsilon e^{i \psi_{2}}\right)$ and (74b) into (92). After a truncation, we obtain

$$
\begin{aligned}
u_{1}= & 2 \operatorname{Re}\left(e^{i \psi_{1}} \varphi_{1}\right) \epsilon+\left(-\operatorname{Re}\left(g_{1110}(0)\right) H_{000001}-\operatorname{Re}\left(g_{2100}(0)\right) H_{000010}\right. \\
& \left.+H_{110000}+\operatorname{Re}\left(e^{2 i \psi_{1}} H_{200000}\right)\right) \epsilon^{2}, \quad \psi_{1} \in[0,2 \pi]
\end{aligned}
$$

and

$$
\begin{aligned}
u_{2}= & 2 \operatorname{Re}\left(e^{i \psi_{2}} \varphi_{2}\right) \epsilon+\left(-\operatorname{Re}\left(g_{0021}(0)\right) H_{000001}-\operatorname{Re}\left(g_{1011}(0)\right) H_{000010}\right. \\
& \left.+H_{001100}+\operatorname{Re}\left(e^{2 i \psi_{2}} H_{002000}\right)\right) \epsilon^{2}, \quad \psi_{2} \in[0,2 \pi] .
\end{aligned}
$$

\subsection{Transcritical-Hopf bifurcation}

Compared with the fold-Hopf bifurcation in Section 6.2, the eigenvalues, eigenfunctions, the homological equation, and the functions $\mathcal{H}, K$ and $R$ remain unchanged. It is only the ODE on the center manifold $\dot{z}$ that changes to the normal form (75). Furthermore, also the critical normal form coefficients for the transcritical-Hopf bifurcation remain the same as for the fold-Hopf bifurcation. Therefore, we proceed only with the parameter-related equations.

Collecting the coefficients of the $z_{0} \beta$ and $z_{1} \beta$ terms in the homological equation we obtain the systems

$$
\begin{aligned}
-A^{\odot \star} j H_{10010} & =A_{1}\left(\varphi_{0}, K_{10}\right) r^{\odot \star}-j \varphi_{0}, \\
-A^{\odot \star} j H_{10001} & =A_{1}\left(\varphi_{0}, K_{01}\right) r^{\odot \star}, \\
\left(i \omega_{0}-A^{\odot \star}\right) j H_{01010} & =A_{1}\left(\varphi_{1}, K_{10}\right)-i \omega_{1} j \varphi_{1} r^{\odot \star}, \\
\left(i \omega_{0}-A^{\odot \star}\right) j H_{01001} & =A_{1}\left(\varphi_{1}, K_{01}\right)-\left(1+i \omega_{2}\right) j \varphi_{1} r^{\odot \star} .
\end{aligned}
$$

Let

$$
K_{\mu}=\gamma_{1 \mu} e_{1}+\gamma_{2 \mu} e_{2}, \quad \mu=(10),(01),
$$

where $e_{1}=(1,0), e_{2}=(0,1)$ and $\gamma_{i \mu}(i=1,2) \in \mathbb{R}$. To determine $\gamma_{i \mu}(i=1,2)$ we substitute (97) into (96). Then by (FSC) we obtain the system

$$
\left(\begin{array}{cc}
p_{0} A_{1}\left(\varphi_{0}, e_{1}\right) & p_{0} A_{1}\left(\varphi_{0}, e_{2}\right) \\
\operatorname{Re}\left(p_{1} A_{1}\left(\varphi_{1}, e_{1}\right)\right) & \operatorname{Re}\left(p_{1} A_{1}\left(\varphi_{1}, e_{2}\right)\right)
\end{array}\right)\left(\begin{array}{cc}
\gamma_{110} & \gamma_{210} \\
\gamma_{101} & \gamma_{201}
\end{array}\right)=\left(\begin{array}{cc}
1 & 0 \\
0 & 1
\end{array}\right)
$$

In order to make the last two systems in (96) consistent we must have that

$$
\begin{aligned}
& \omega_{1}=\operatorname{Im}\left(p_{1} A_{1}\left(\varphi_{1}, K_{10}\right)\right), \\
& \omega_{2}=\operatorname{Im}\left(p_{1} A_{1}\left(\varphi_{1}, K_{01}\right)\right) .
\end{aligned}
$$




\subsubsection{Neimark-Sacker predictors}

The predictors for the Hopf and transcritical bifurcation curves, as well as those for the Neimark-Sacker bifurcation curves (including the cycle periods), can be easily obtained using the asymptotics from Sections 5.4.1 and 5.4.2. In particular, to approximate the periodic orbits along the Neimark-Sacker curves, we substitute $z_{1}=\epsilon e^{i \psi}$ and (77) into (86). This gives the following linear approximations:

$$
u=\left(\mp \sqrt{\frac{g_{011}(0)}{g_{200}(0)}} \varphi_{0}+2 \operatorname{Re}\left(e^{i \psi} \varphi_{1}\right)\right) \epsilon \quad \psi \in[0,2 \pi] .
$$

\section{Computation of derivatives for discrete DDEs}

All predictors described in the previous sections are implemented in version 3.2a of DDE-BifTool for models of the type (1). The discrete DDE (1) is a particular instance of (28) with $h=\tau_{m}$ and

$$
F(\varphi, \alpha)=f(\Xi \varphi, \alpha),
$$

where the linear evaluation operator $\Xi: X \rightarrow \mathbb{R}^{n \times(m+1)}$ is defined by

$$
\Xi \varphi:=\left(\varphi\left(-\tau_{0}\right), \varphi\left(-\tau_{1}\right), \ldots, \varphi\left(-\tau_{m}\right)\right) .
$$

with the convention $\tau_{0}:=0$. In particular, by the chain rule,

$$
D_{1} F(0,0) \varphi=D_{1} f(0,0) \Xi \varphi=\sum_{j=0}^{m} D_{1, j} f(0,0) \varphi\left(-\tau_{j}\right), \quad \varphi \in X,
$$

with $M_{j}:=D_{1, j} f(0,0) \in \mathbb{R}^{n \times n}$ the partial derivative of $f$ at the origin with respect to its $j$ th state argument. So, if (1) has a equilibrium at the origin for $\alpha=0$, then the linear part of the splitting (11) at $\alpha=0$ is precisely the right-hand side of the above equation. Therefore $\zeta:[0, h] \rightarrow \mathbb{R}^{n \times n}$ must be such that

$$
\langle\zeta, \varphi\rangle=\sum_{j=0}^{m} M_{j} \varphi\left(-\tau_{j}\right), \quad \forall \varphi \in X .
$$

Hence $\zeta$ has jump discontinuities $M_{j}$ at the points $\tau_{j}$ for $j=0, \ldots, m$ and is constant otherwise. So, in this case the characteristic matrix (20) is given by

$$
\Delta(z)=z I-\sum_{j=0}^{m} M_{j} \mathrm{e}^{-z \tau_{j}}, \quad z \in \mathbb{C} .
$$

The multilinear forms appearing in (78) can be expressed in terms of the derivatives of the function $f: \mathbb{R}^{n \times(m+1)} \times \mathbb{R}^{p} \rightarrow \mathbb{R}^{n}$ from (1). For $r, s \geq 0$ with $r+s \geq 1$ the mixed derivative of order $r+s$ of $f$ at $(0,0)$ is an $(r+s)$-linear form on $\left[\mathbb{R}^{n \times(m+1)}\right]^{r} \times\left[\mathbb{R}^{p}\right]^{s}$, with the understanding that at most one factor may be absent in case $r=0$ or $s=0$. Let $Q, Q^{1}, \ldots, Q^{r}$ be matrices in $\mathbb{R}^{n \times(m+1)}$ and let $\alpha, \alpha^{1}, \ldots, \alpha^{s}$ be vectors in $\mathbb{R}^{p}$. Then this derivative acts as

$$
\begin{aligned}
& D_{1}^{r} D_{2}^{s} f(0,0)\left(Q^{1}, \ldots, Q^{r}, \alpha^{1}, \ldots, \alpha^{s}\right)= \\
& \qquad\left.\sum_{j, k, \ell} \frac{\partial^{r+s} f(Q, \alpha)}{\partial q_{j_{1} k_{1}} \ldots \partial q_{j_{r} k_{r}} \partial \alpha_{\ell_{1}} \ldots \partial \alpha_{\ell_{s}}}\right|_{(Q, \alpha)=(0,0)} q_{j_{1} k_{1}}^{1} \cdots q_{j_{r} k_{r}}^{r} \alpha_{\ell_{1}}^{1} \cdots \alpha_{\ell_{s}}^{s},
\end{aligned}
$$

where the multidimensional sum runs over

$$
1 \leq j_{1}, \ldots, j_{r} \leq n, \quad 0 \leq k_{1}, \ldots, k_{r} \leq m, \quad 1 \leq \ell_{1}, \ldots, \ell_{s} \leq p .
$$


The multilinear forms appearing in (78), as well as (80), are computed from (99) by composition with $\Xi$ from $(98)$ as

$$
D_{1}^{r} D_{2}^{s} F(0,0)\left(\varphi_{1}, \ldots, \varphi_{r}, \alpha_{1}, \ldots, \alpha_{s}\right)=D_{1}^{r} D_{2}^{s} f(0,0)\left(\Xi \varphi_{1}, \ldots, \Xi \varphi_{r}, \alpha_{1}, \ldots, \alpha_{s}\right),
$$

for $\varphi_{1}, \ldots, \varphi_{r} \in X$ and $\alpha_{1}, \ldots, \alpha_{s} \in \mathbb{R}^{p}$. For given $r$ and $s$ the multidimensional array of partial derivatives inside the sum in (99) is of course symmetric under permutation of the state indices $j_{1} k_{1}, \ldots, j_{r} k_{r}$ and the parameter indices $\ell_{1}, \ldots, \ell_{s}$. This can be exploited for efficient storage and access.

\section{Examples}

In this section we will demonstrate the correctness of the normal form coefficients and the accuracy of the predictors in four different models. We do this twofold. Firstly, by comparing the predictors in parameter-space with the computed in DDE-BifTool bifurcation curves, and, secondly, by performing simulations near the bifurcation point under consideration. The simulation is done either with the build-in routine dde23 of MATLAB or with the Python package pydelay [24]. The latter gives significant speed performance when considering simulation over longer time intervals. This usually is the case when one wants to demonstrate the existence of stable invariant manifolds. Since in this section only the main results are given, we provide details (including simulation results) in the Supplement. Furthermore, the source code of the examples has been included into the DDE-BifTool software package. This will hopefully provide a good starting point when considering other models.

\subsection{Generalized Hopf bifurcation in a coupled FHN neural system with delay}

In [65] the following system is considered

$$
\left\{\begin{array}{l}
\dot{u}_{1}(t)=-\frac{u_{1}^{3}(t)}{3}+(c+\alpha) u_{1}^{2}(t)+d u_{1}(t)-u_{2}(t)+2 \beta f\left(u_{1}(t-\tau)\right), \\
\dot{u}_{2}(t)=\varepsilon\left(u_{1}(t)-b u_{2}(t)\right) .
\end{array}\right.
$$

Here $\left(u_{1}, u_{2}\right)$ is the completely synchronous solution of the three coupled FitzHugh-Nagumo (FHN) neuron system

$$
\left\{\begin{array}{l}
\dot{u}_{1}(t)=-\frac{u_{1}^{3}(t)}{3}+(c+\alpha) u_{1}^{2}(t)+d u_{1}(t)-u_{2}(t)+\beta\left[f\left(u_{3}(t-\tau)\right)+f\left(u_{5}(t-\tau)\right)\right], \\
\dot{u}_{2}(t)=\varepsilon\left(u_{1}(t)-b u_{2}(t)\right), \\
\dot{u}_{3}(t)=-\frac{u_{3}^{3}(t)}{3}+(c+\alpha) u_{3}^{2}(t)+d u_{3}(t)-u_{4}(t)+\beta\left[f\left(u_{3}(t-\tau)\right)+f\left(u_{5}(t-\tau)\right)\right], \\
\dot{u}_{4}(t)=\varepsilon\left(u_{3}(t)-b u_{4}(t)\right), \\
\dot{u}_{5}(t)=-\frac{u_{5}^{3}(t)}{3}+(c+\alpha) u_{5}^{2}(t)+d u_{5}(t)-u_{6}(t)+\beta\left[f\left(u_{1}(t-\tau)\right)+f\left(u_{3}(t-\tau)\right)\right], \\
\dot{u}_{6}(t)=\varepsilon\left(u_{5}(t)-b u_{6}(t)\right),
\end{array}\right.
$$

where $\alpha, \beta$ measure the synaptic strength in self-connection and neighborhood-interaction, respectively. The parameters $b$ and $\epsilon$ are assumed to be positive such that $0<b<1$ and $0<\epsilon \ll 1$. The function $f$ is a sufficiently smooth sigmoid amplification function and $\tau>0$ represents the time delay in signal transmission. For the derivation of (100) from the system (101), as well as for stability conditions of the completely synchronous solution, we refer to [65]. In that article a generalized Hopf point was analyzed using the traditional formal adjoint method and the two-step center manifold reduction, 
see [31]. Numerical simulations where made to confirm their results. For this $(\beta, \alpha)$ are taken as the unfolding parameters and the parameters

$$
b=0.9, \quad \varepsilon=0.08, \quad c=2.0528, \quad d=-3.2135, \quad \tau=1.7722
$$

are fixed. The sigmoid amplification function $f(u)=\tanh (u)$ is used.

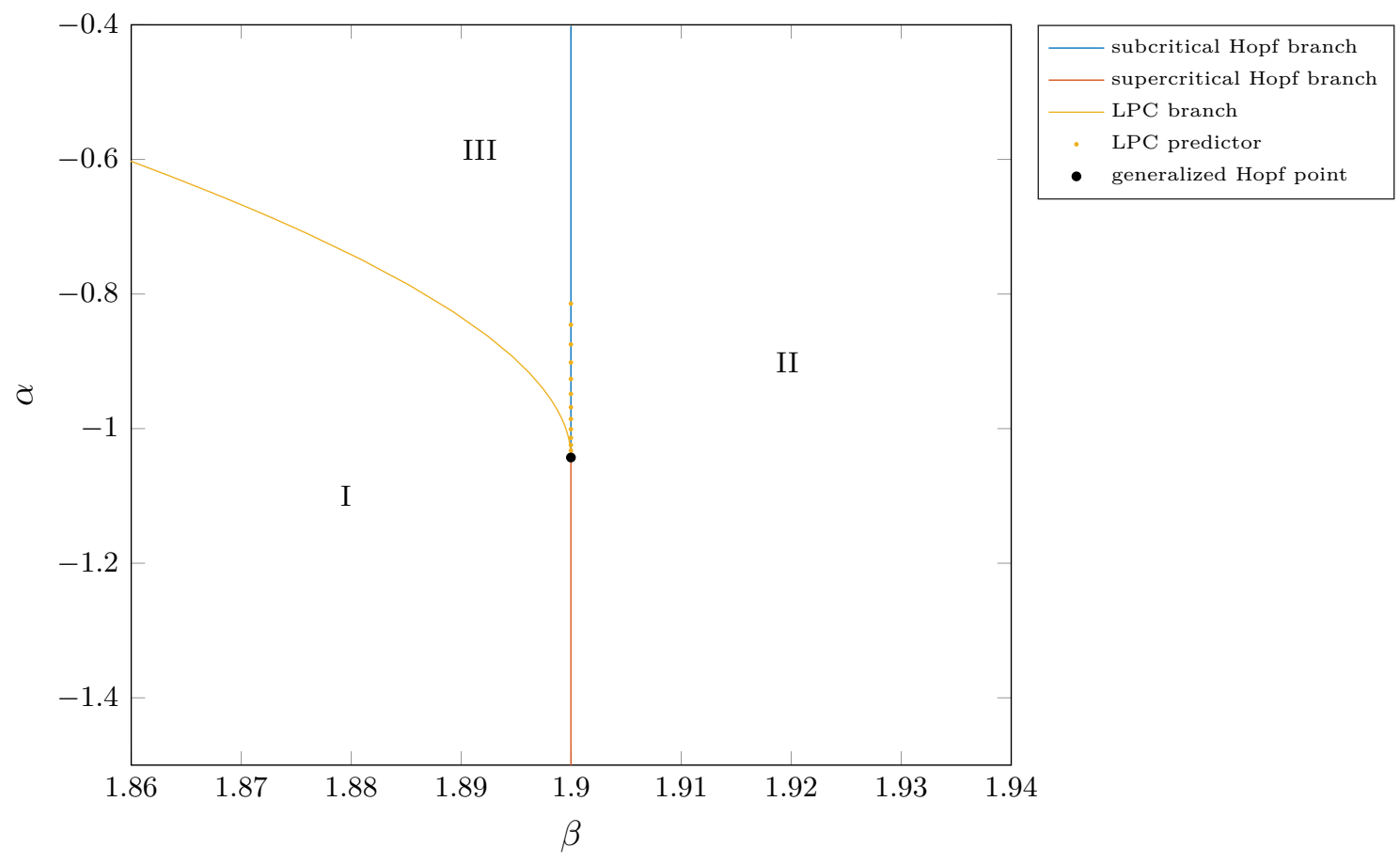

Figure 1: Bifurcation diagram near the generalized Hopf point in the system (100) with unfolding parameters $(\beta, \alpha)$. The bifurcation curves are nearly identical to those in the bifurcation diagram of the topological normal form as presented in [43, page 314].

According to [65], a generalized Hopf point is present at the origin with the parameter values $(\beta, \alpha)=(1.9,-0.9710)$. We took this point and calculated its stability and the corresponding normal form coefficients. Although we do confirm that the point under consideration is a Hopf point, the first Lyapunov coefficient does not vanish and we conclude that the point cannot be a generalized Hopf point. However, the simulation in [65] do suggest a generalized Hopf point for nearby parameter values. Therefore we continued the Hopf point in $(\beta, \alpha)$. Then a generalized Hopf point is located at $(\beta, \alpha)=(1.9,-1.0429)$ with negative second Lyapunov coefficient $\ell_{2}(0)=-15.6733$, indicating the existence of a stable steady state inside a unstable cycle, which in turn is located inside a stable cycle. We remark that the second Lyapunov coefficient found in [65] is positive. This contradicts the simulation of the dynamics made in the same article. Indeed when the second Lyapunov coefficient is positive a time-reversal must be taking into account when considering the bifurcation diagram in the case the second Lyapunov coefficient is negative, see [43]. Then the situation of a stable steady state inside a stable cycle (separated by an unstable cycle) does not occur.

Using the predictors from Section 5.1 the fold and LPC bifurcation curves emanating from the generalized Hopf point. In Figure 1 the resulting bifurcation diagram is shown. 


\subsection{Fold-Hopf bifurcation of the Rose-Hindmarsh model with time delay}

In [48] a Rose-Hindmarsh model [33, 34] with time delay in the self-feedback process, which takes the form

$$
\left\{\begin{array}{l}
\dot{x}(t)=y(t)-a x^{3}(t)+b x^{2}(t-\tau)-c z(t)+I_{a p p}, \\
\dot{y}(t)=c-d x^{2}(t)-y(t) \\
\dot{z}(t)=r(S(x(t)-\chi)-z(t))
\end{array}\right.
$$

is considered. Here $x$ represents membrane potential, $y$ represents a recovery variable, $z$ denotes the adaption current, and $a, b, c, d>0, S$ and $\chi$ are real constants. The external current $I_{a p p}$ and $r$ are control parameters, and $\tau$ denotes the synaptic transmission delay. The constants $a, b, c, d, \chi$ and $r$ are fixed. Let $\left(x_{\star}, y_{\star}, z_{\star}\right)$ be a steady state of (102), then

$$
y_{\star}=c-d x_{\star}^{2}, \quad z_{\star}=S\left(x_{\star}-\chi\right) .
$$

The conditions for a fold-Hopf bifurcation have been derived in [48] analytically. Indeed, let $S$ be arbitrary and set

$$
\begin{aligned}
x_{\star}= & \frac{1}{3 a}\left(b-d \pm \sqrt{(b-d)^{2}-3 a c S}\right), \\
I_{a p p}= & x_{\star}^{2}\left(a x_{\star}-b+d\right)+c\left(S\left(x_{\star}-\chi\right)-1\right), \\
A= & x_{\star}^{2}\left(\left(3 a x_{\star}+2 d\right)^{2}-4 b^{2}\right)-2 r x_{\star}\left(2 d x_{\star}-1\right)\left(3 a x_{\star}-2 b+2 d\right) \\
& \quad+r^{2}\left(4 d x_{\star}\left(-2 b x_{\star}+d x_{\star}-1\right)+1\right), \\
B= & 9 a^{2} x_{\star}^{4}+2 r x_{\star}\left(3 a x_{\star}-2 b+2 d\right)-4 b^{2} x_{\star}^{2}-4 d x_{\star}+r^{2}+1, \\
\omega_{1,2}= & \sqrt{-B \pm \sqrt{B^{2}-4 A}} .
\end{aligned}
$$

Then a fold-Hopf bifurcation occurs when

$$
\tau= \begin{cases}\frac{1}{\omega_{1,2}}(\arcsin Y+2 k \pi), & Z \geq 0, \\ \frac{1}{\omega_{1,2}}(\pi-\arcsin Y+2 k \pi), & Z \leq 0,\end{cases}
$$

where $k=0,1,2, \ldots$ and

$$
\begin{aligned}
& Y=\frac{\omega_{1,2}}{2 b}\left(\frac{r\left(2 b-2 d-3 a x_{\star}\right)}{r^{2}+\omega_{1,2}^{2}}+\frac{2 d}{\omega_{1,2}^{2}+1}-\frac{1}{x_{\star}}\right), \\
& Z=\frac{\omega_{1,2}}{2 b}\left(\frac{r^{2}\left(2 b-2 d-3 a x_{\star}\right)}{r^{2}+\omega_{1,2}^{2}}+\frac{2 d}{\omega_{1,2}^{2}+1}+3 a x_{\star}\right) .
\end{aligned}
$$

In [48] the parameters values

$$
a=1.0, \quad b=3.0, \quad c=1.0, \quad d=5.0, \quad \chi=-1.6, \quad r=0.001
$$

are fixed. It follows that a fold-Hopf bifurcation is located at

$$
x_{\star}=0.1308, \quad S=-0.57452592, \quad \tau=5.768830916,
$$

and $I_{a p p},\left(y_{\star}, z_{\star}\right)$ given by (104) and (103), respectively. To unfold the singularity the 'parameters' $\left(x_{\star}, S\right)$ are used, see [48]. Here we will take the more natural unfolding parameter $\left(I_{a p p}, S\right)$. Calculating the stability with DDE-BifTool gives the eigenvalues

$$
0.001 \pm 1.0081 i, \quad-0.000+0.000 i .
$$


All other eigenvalues lie in the open left half of the complex plane. Calculating the normal form coefficients reveals that

$$
s=\operatorname{sgn}\left(g_{200}(0) g_{011}(0)\right)=\operatorname{sgn}(1.8487 \mathrm{e}-05), \quad \theta(0)=\frac{\operatorname{Re}\left(g_{110}(0)\right)}{g_{200}(0)}=-139.0315
$$

and

$$
e(0)=\operatorname{Re}\left[g_{210}(0)+g_{110}(0)\left(\frac{\operatorname{Re} g_{021}(0)}{g_{011}(0)}-\frac{3}{2} \frac{g_{300}(0)}{g_{200}(0)}+\frac{g_{111}(0)}{2 g_{011}(0)}\right)-\frac{g_{021}(0) g_{200}(0)}{g_{011}}\right]=15.6941 .
$$

Since $s=1$ and $\theta(0)<0$, a global bifurcation curve or invariant tori are present for parameters sufficiently close to the bifurcation, see [43, page 342]. However, since the sign of $e(0)$ is positive the tori are unstable. Thus according to our analysis the simulated torus in [48] cannot be attributed to the fold-Hopf bifurcation.

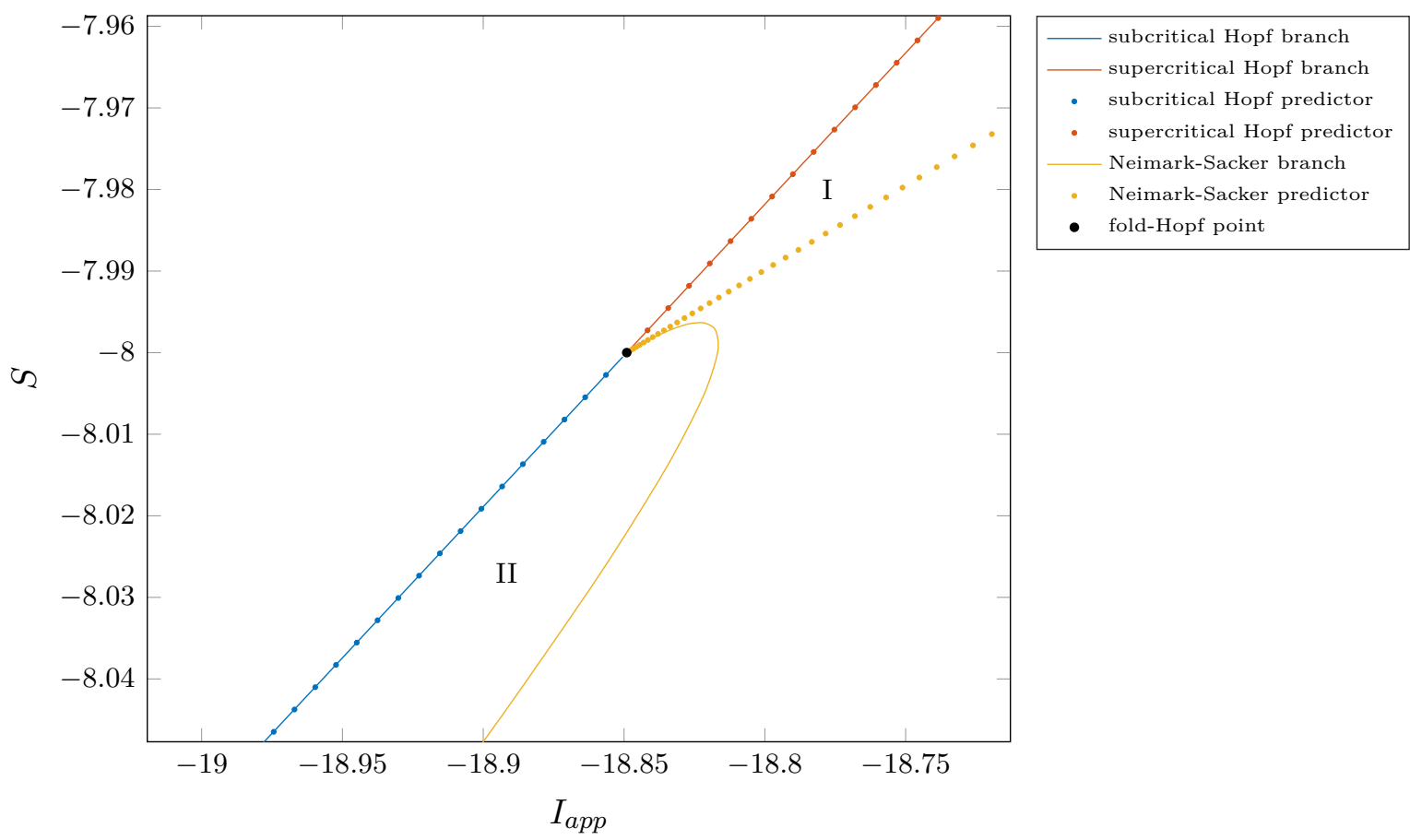

Figure 2: Bifurcation diagram near the fold-Hopf point in $(102)$ with $(r, S)=(1.4,-8)$. The fold branch is not included here since it is indistinguishable from the Hopf curve at this scale.

For demonstration purposes, we take the parameters $r=1.4$ and $S=-8$, while keeping the other parameters as in (105). Then a fold-Hopf bifurcation is located at $x_{\star}=1.0972, \tau=0.9402, I_{a p p}$ as in $(104)$, and $\left(y_{\star}, z_{\star}\right)$ given by (103). The leading eigenvalues become

$$
0.000 \pm 5.6042 i, \quad 0.000+0.000 i
$$

while the normal form coefficients are given by

$$
s=\operatorname{sgn}(1.7700), \quad \theta(0)=-0.1569 \quad \text { and } \quad e(0)=-0.0378 .
$$

Thus the sign of $s$ and $\theta(0)$ remain unchanged. However, since the sign of $e(0)$ is negative, there is a time reversal to take into account. Therefore, we expect a stable torus to be present for nearby parameter values. Using the predictors from Section 5.2, we successfully continued the fold, Hopf, and Neimark-Sacker bifurcation curves emanating from the point, see Figure 2. 


\subsection{Hopf-Hopf and generalized Hopf bifurcations in Active control system}

Active control system is used to control the response of structures to internal or external excitation. The mathematical model with time delay can be described as follows [50]

$$
m \ddot{x}(t)+c \dot{x}(t)+k x(t)+u x(t-\tau)+v \dot{x}(t-\tau)=\tilde{f}(t) .
$$

Here $x(t)$ is the displacement of the controlled system, $m>0$ is the mass, $c$ and $k$ are the damping and the stiffness, respectively, $\tau$ is the time delay represented in the relative displacement feedback loop and in the relative velocity feedback loop, $u$ and $v$ are feedback strengths, respectively, and $\tilde{f}$ represents the external excitation. Let $t^{*}=\sqrt{k / m} t, \zeta=c / 2 m \sqrt{m / k}, g_{u}=u / k, g_{v}=v / m \sqrt{m / k}$ and $f(t)=\tilde{f}(t) / k$. Then equation (107) becomes

$$
\ddot{x}(t)+2 \zeta \dot{x}(t)+x(t)+g_{u} x(t-\tau)+g_{v} \dot{x}(t-\tau)=f(t),
$$

where the asterisks are omitted for simplicity. Following [16] and [50] we consider the case when $f$ is replaced by a nonlinear position time delay feedback given by $\beta x^{3}(t-\tau)$, see also [63]. As in [16] we fix the parameters

$$
g_{u}=0.1, \quad g_{v}=0.52, \quad \beta=0.1
$$

and take $\zeta$ and $\tau$ as control parameters. Let $\dot{x}(t)=y(t)$, then we obtain

$$
\left\{\begin{array}{l}
\dot{x}(t)=\tau y(t), \\
\dot{y}(t)=\tau\left(-x(t)-g_{u} x(t-1)-2 \zeta y(t)-g_{v} y(t-1)+\beta x^{3}(t-1)\right) .
\end{array}\right.
$$

Here the delay is scaled by using the transformation of time $t \rightarrow t / \tau$. In this way the delay can treated as an ordinary parameter.

The trivial equilibrium undergoes a Hopf-Hopf bifurcation at the parameter values

$$
\left(\zeta_{c}, \tau_{c}\right)=(-0.016225,5.89802)
$$

see [16] for the derivation. Using DDE-BifTool we manually construct the Hopf-Hopf point and compute its stability and normal form coefficients. We obtain the eigenvalues $0.0000 \pm 4.5275 i$ and $-0.0000 \pm$ 7.6449i. The quadratic critical normal form coefficients are

$$
\begin{array}{ll}
g_{2100}(0)=-0.0915+0.1214 i, & g_{1110}(0)=0.2151+0.3876 i \\
g_{1011}(0)=-0.3084+0.4096 i, & g_{0021}(0)=0.1813+0.3268 i
\end{array}
$$

From

$$
\left(\operatorname{Re} g_{2100}(0)\right)\left(\operatorname{Re} g_{0021}(0)\right)=-0.0166<0,
$$

we conclude that this Hopf-Hopf bifurcation is of 'difficult' type, see [43]. Furthermore, since the quantities

$$
\theta=\theta(0)=\frac{\operatorname{Re} g_{1011}(0)}{\operatorname{Re} g_{0021}(0)}=-1.7009, \quad \delta=\delta(0)=\frac{\operatorname{Re} g_{1101}(0)}{\operatorname{Re} g_{2100}(0)}=-2.3517
$$

are such that $\theta<0, \delta<0, \theta \delta>1$ it follows that we are in case VI. We continue the NeimarkSacker and Hopf bifurcation curses emanating from the Hopf-Hopf point using the predictors from Section 5.3. In Figure 3 a close-up is given near the Hopf-Hopf point comparing the computed curves with the predictors in parameter space.

Using the detection capabilities of DDE-BifTool one additional Hopf-Hopf point and three generalized Hopf points are located on the continued Hopf branches. The normal form coefficients of the second Hopf-Hopf point are such that

$$
\left(\operatorname{Re} g_{2100}(0)\right)\left(\operatorname{Re} g_{0021}(0)\right)=1.7331 \mathrm{e}-04>0
$$




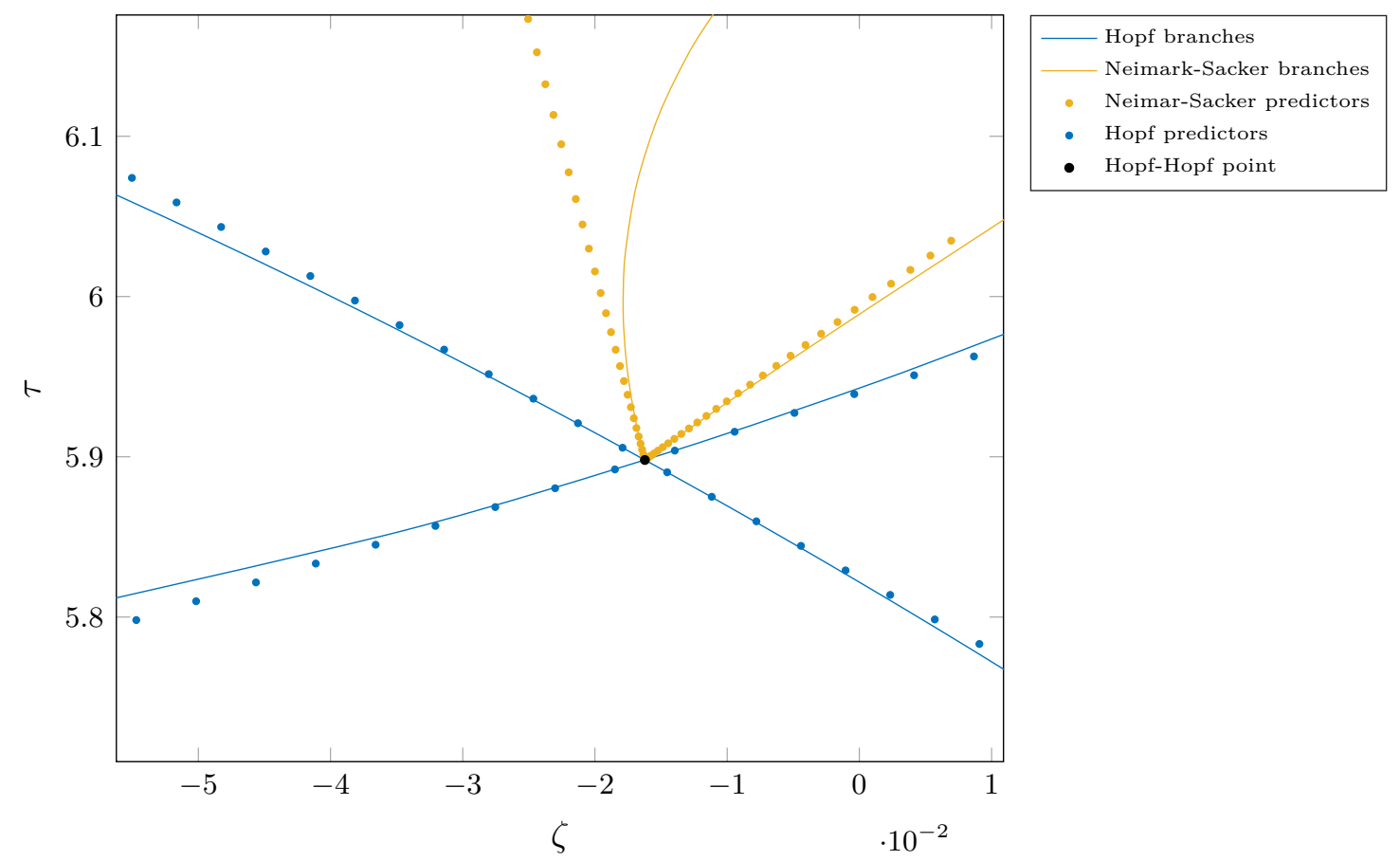

Figure 3: Bifurcation diagram near the Hopf-Hopf point at parameter values (109) in an active control system with time delay given by (108). There are two supercritical Hopf curves (blue) and two Neimark-Sacker curves (yellow). We see that the predictors (dotted) give good approximations near the codimension two point. 
and

$$
\theta \geq \delta>0, \quad \theta \delta>1 .
$$

We conclude that we are in case I of the 'simple' type, see [43, page 360]. Therefore, no stable invariant two-dimensional torus is predicted for nearby parameter values, only two stable period orbits expected. Using the predictors from Sections 5.1 and 5.3 we can easily continue the codimension one cycle bifurcations from the located degenerate Hopf points, showing complicated bifurcation diagram in Figure 4.

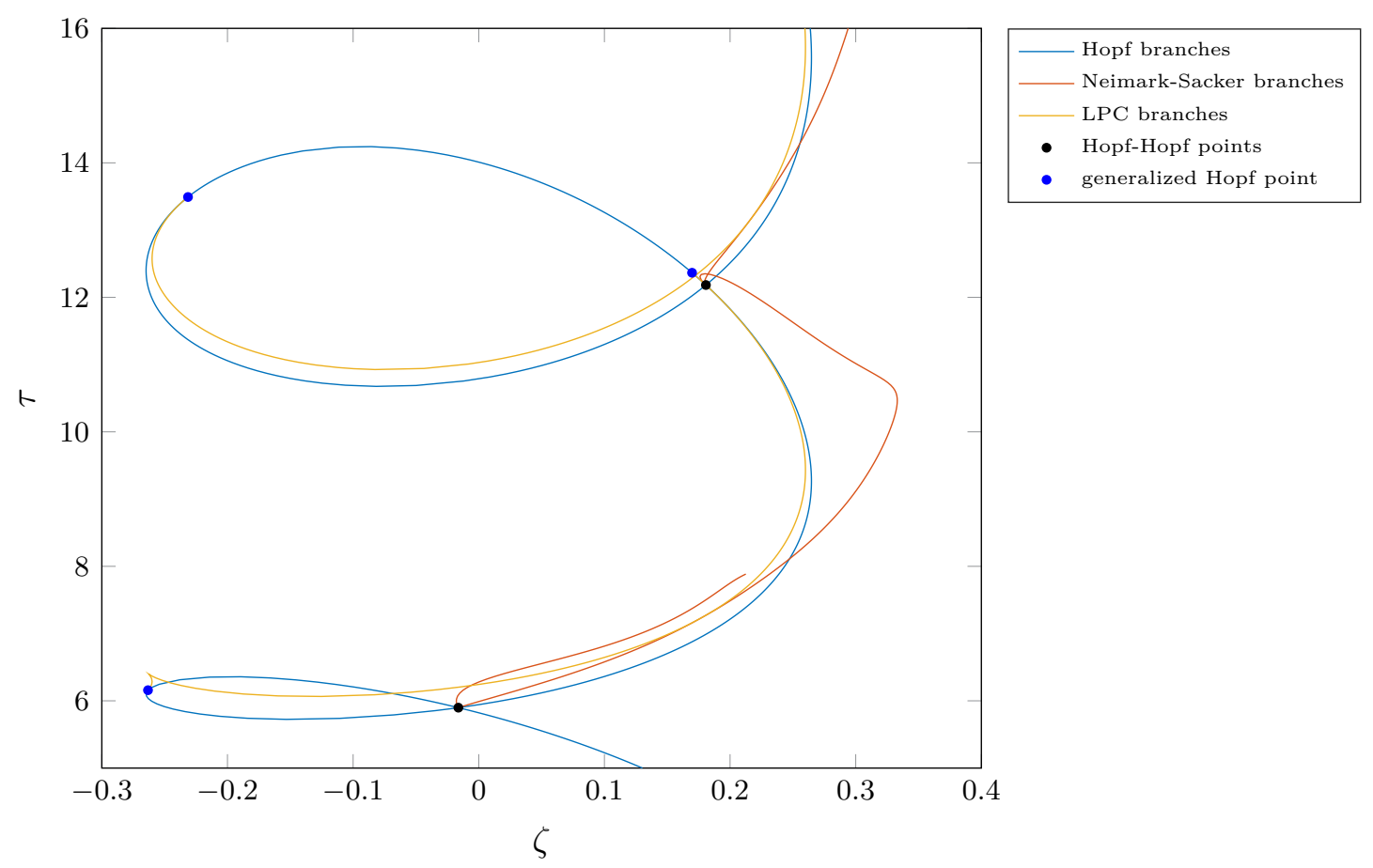

Figure 4: Bifurcation diagram obtained by continuing Hopf-Hopf, Neimark-Sacker and LPC bifurcation curves in the active control system (108) using the predictors from Sections 5.1 and 5.3 combined with the continuation capabilities from DDE-BifTool. Two Hopf-Hopf points are connected by a NeimarkSacker bifurcation curve. Also two of the three generalized Hopf points are connected by a single LPC curve.

\subsection{Transcritical-Hopf bifurcation in Van der Pol's oscillator with delayed position and velocity feedback}

In [3] a generalization of Van der Pol's oscillator with delayed feedback

$$
\ddot{x}(t)+\varepsilon\left(x^{2}(t)-1\right) \dot{x}(t)+x(t)=g(\dot{x}(t-\tau), x(t-\tau)), \quad 0<\tau<\infty,
$$

is considered. Here $g \in C^{3}$ satisfies the conditions $g(0,0)=0, g_{\dot{x}}(0,0)=a$ and $g_{x}(0,0)=b$. The linearization of equation (110) around the trivial solution $x=0$ gives

$$
\ddot{x}(t)-\varepsilon \dot{x}(t)+x(t)=a \dot{x}(t-\tau)+b x(t-\tau) .
$$

From which we obtain the characteristic equation

$$
\Delta(\lambda, \tau)=\lambda^{2}-\varepsilon \lambda+1-(a \lambda+b) e^{-\lambda \tau}=0 .
$$


Let

$$
b=1, \quad \tau=\tau_{0} \neq \varepsilon+a, \quad \varepsilon^{2}-a^{2}<2,
$$

then the characteristic equation has a simple zero and a pair of purely imaginary roots $\lambda= \pm i \omega_{0}$. Here $\omega_{0}$ and $\tau_{0}$ are defined by

$$
\omega_{0}=\sqrt{2-\varepsilon^{2}+a^{2}}, \quad \tau_{0}=\frac{1}{\omega_{0}} \arccos \left(\frac{1-(1+\varepsilon a) \omega_{0}^{2}}{a^{2} \omega_{0}^{2}+1}\right),
$$

see [3, Proposition 2.1]. We set the function $g$ to

$$
\begin{aligned}
g(\dot{x}(t-\tau), x(t-\tau))= & \left(1+\mu_{1}\right) x(t-\tau)-0.2 \dot{x}(t-\tau)-0.2 x(t-\tau)^{2} \\
& -0.2 x(t-\tau) \dot{x}(t-\tau)-0.2 x(t-\tau)^{2}+0.5 x(t-\tau)^{3}
\end{aligned}
$$

and $\varepsilon=0.3$. Then the conditions (111) are satisfied and

$$
\omega_{0} \approx 1.396424004376894, \quad \tau_{0} \approx 1.757290761249588 .
$$

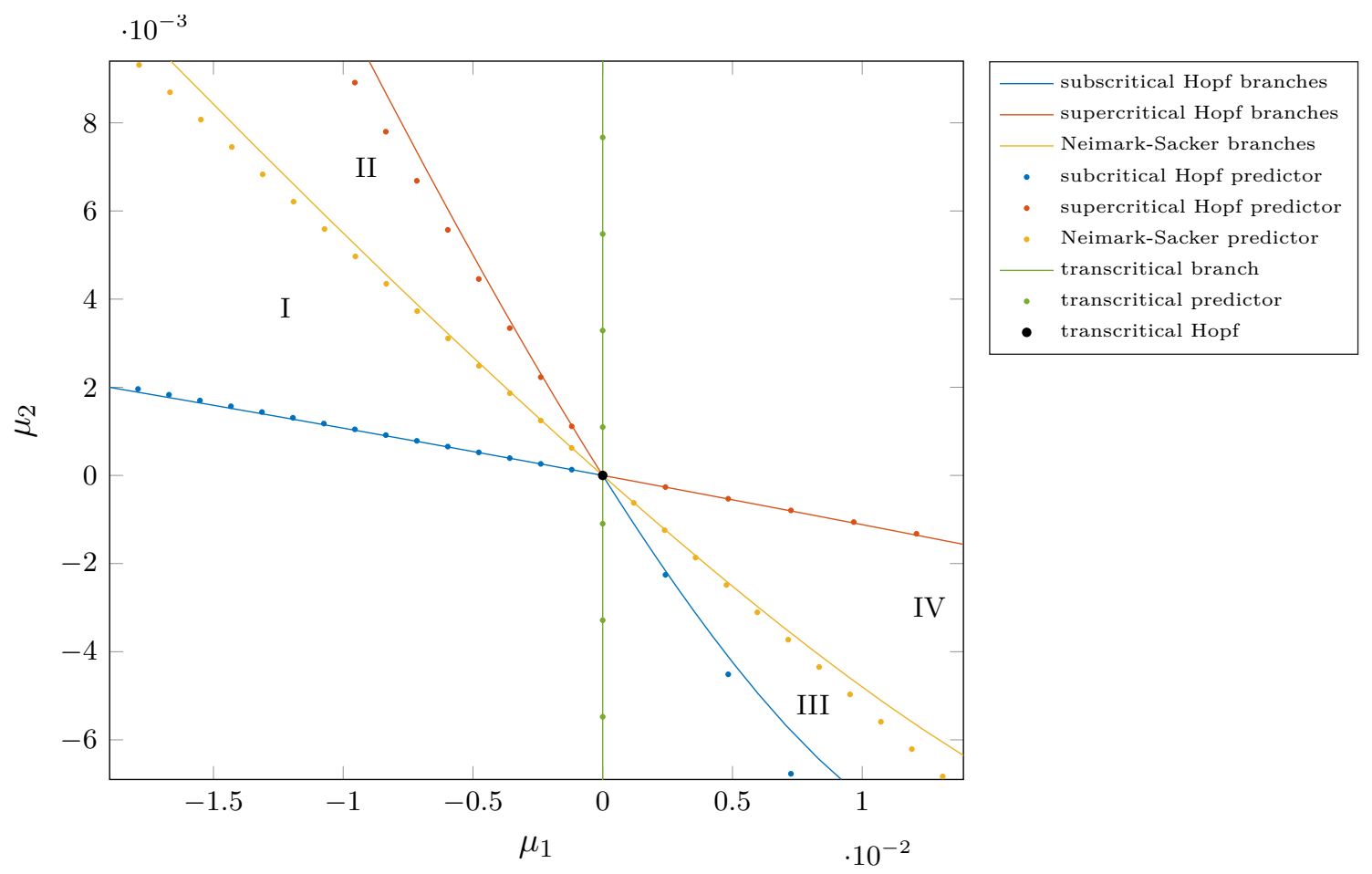

Figure 5: Bifurcation diagram near the transcritical-Hopf bifurcation in the delayed Van der Pol's oscillator given by (113). There are two supercritical Hopf curves (blue), two subcritical Hopf curves (red), two Neimark-Sacker curves (yellow) and one transcritical curve (green). We see that the predictors (dotted) give good approximation for nearby values.

To analyze the system with DDE-BifTool we set $y(t)=\dot{x}(t)$ and transform the time with $t \rightarrow t / \tau$ to obtain the two-component system

$$
\left\{\begin{aligned}
\dot{x}(t)= & \left(\tau_{0}+\mu_{2}\right) y(t), \\
\dot{y}(t)= & \left(\tau_{0}+\mu_{2}\right)\left[-x(t)-\varepsilon\left(x^{2}(t)-1\right) y(t)+\left(1+\mu_{1}\right) x(t-1)-0.2 y(t-1)\right. \\
& \left.\quad-0.2 x^{2}(t-1)-0.2 x(t-1) y(t-1)-0.2 y^{2}(t-1)+0.5 x^{3}(t-1)\right] .
\end{aligned}\right.
$$


Here we introduced the unfolding parameters $\left(\mu_{1}, \mu_{2}\right):=\left(b-1, \tau-\tau_{0}\right)$ to translate the singularity to the origin. One immediately sees that the trivial equilibrium $(\dot{x}, x)=(0,0)$ is an equilibrium for all parameter values $\left(\mu_{1}, \mu_{2}\right)$. Therefore, the parameter-dependent normal form for the generic foldHopf cannot be used here. Instead the normal form for the transcritical-Hopf bifurcation must be used. Using DDE-BifTool we compute the stability and the normal form coefficients. The leading eigenvalues are $0.000+0.000 i$ and $-0.000+2.4539 i$, where $2.4539 \approx \omega_{0} \tau_{0}$, see (112). Furthermore, the normal form coefficients are such that

$$
g_{011}(0) \times \operatorname{Re}\left(g_{110}(0)\right)=0.4241 \times \operatorname{Re}(-0.1337+0.2672 i)<0 .
$$

Therefore, there are two Neimark-Sacker bifurcation curves predicted, see Section 5.4. Using the predictors from Section 5.4 we continue the transcritical, Hopf and Neimark-Sacker bifurcation curves emanating from the transcritical-Hopf bifurcation point. In Figure 5 the bifurcation diagram is shown.

\section{Concluding remarks}

We have provided explicit formulas for the normal form coefficients needed to initialize codimension one equilibrium and nonhyperbolic cycle bifurcations emanating from generalized Hopf, fold-Hopf, Hopf-Hopf and transcritical-Hopf points in DDEs. Applications to four different models were given, confirming the correctness of the derivation of the normal form coefficients and the asymptotics. A paper providing a second-order predictor for the homoclinic orbits emanating from the generic and transcritical codimension two Bogdanov-Takens bifurcations in DDEs, along the lines of [45], is in preparation.

Our proof of the existence of a smooth parameter-dependent center manifold is given in the general context of perturbation theory for dual semigroups (sun-star calculus). Consequently the applicability of this result extends beyond classical DDEs, although here we did restrict to the case of an eventually compact $\mathcal{C}_{0}$-semigroup on a sun-reflexive state space. It follows that the results from Sections 3.2 to 3.5 are valid as well for other classes of delay equations such as renewal equations (also known as Volterra functional equations) and systems of mixed type [11].

Furthermore, in $[15,59]$ the technique was used to calculate the critical normal form coefficients for Hopf and Hopf-Hopf bifurcations occurring in neural field models with propagation delays. For these models sun-reflexivity is lost, which is typical for delay equations in abstract spaces or with infinite delay. However, it is often possible to overcome this functional analytic complication, so dual perturbation theory can still be employed successfully $[12,13,38,59]$. It has also been used in the context of semilinear hyperbolic systems [47].

It is demonstrated - at a formal level - in [55] that the normalization technique described in Section 4 still works for DDEs with state-dependent delays. However, as already mentioned in Remark 21, for DDEs the nonlinearity generally does not depend differentiably on the delay parameters. Therefore, in the case of state-dependent DDEs it is generally not possible to justify differentiation of the nonlinearity with respect to the state, let alone to rely on higher order smoothness. So, as far as we know there is still no proof of the validity of the normalization technique for state-dependent DDEs.

Returning to the setting of classical DDEs, the most obvious next challenge is to derive normal forms for bifurcations of periodic orbits by generalizing $[9,10,44]$ to DDEs. The resulting formulas can then be implemented in DDE-BifTool to facilitate numerical bifurcation analysis of periodic orbits in classical DDEs.

\section{Acknowledgments}

The authors would like to thank Prof. Odo Diekmann (Utrecht University) for very useful discussions on parameter-dependent perturbation of linear semigroups. We also thank Prof. Peter De Maesschalck (Hasselt University) for supporting this research project. 


\title{
SUPPLEMENTARY MATERIALS FOR: Switching to nonhyperbolic cycles from codimension two bifurcations of equilibria of delay differential equations
}

\author{
M.M. Bosschaert* $\quad$ S.G. Janssens ${ }^{\dagger} \quad$ Yu.A. Kuznetsov ${ }^{\ddagger}$
}

March 19, 2019

In this supplement we provide walkthroughs of the examples given in Section 8 with DDE-BifTool ${ }^{1}$ [21]. These walkthroughs enable other researchers to reproduce the results obtained in the main text.

Additionally, we will show the code used for simulation near the bifurcations points under consideration. Either using the build-in routine dde23 from MATLAB [53] or the Python package pydelay ${ }^{2}$ [24]. Other DDE models, undergoing one of the degenerate Hopf bifurcations treated in this paper, can easily be studied by making minor modifications to the given code.

The focus will be on the initialization and continuation of the various codimension one equilibrium and cycles bifurcation curves emanating from the degenerate Hopf points and on simulation near the bifurcation points. For a complete overview of the capabilities and functionality for DDE-BifTool, we refer to the online tutorials files and also the manual and the references therein.

All code has been included into the DDE-BifTool package version 3.2a on the SourceForge repository and can be executed without the need to copy and paste. Note that the code is tested on MATLAB 2018b and GNU Octave 4.2.2. Different results may occur with other versions of MATLAB and GNU Octave.

\section{S1 Generalized Hopf bifurcation in a coupled FHN neural sys- tem with delay}

A completely synchronous solution of the three coupled FitzHugh-Nagumo (FHN) neuron system is given by the system

$$
\left\{\begin{array}{l}
\dot{u}_{1}(t)=-\frac{u_{1}^{3}(t)}{3}+(c+\alpha) u_{1}^{2}(t)+d u_{1}(t)-u_{2}(t)+2 \beta f\left(u_{1}(t-\tau)\right), \\
\dot{u}_{2}(t)=\varepsilon\left(u_{1}(t)-b u_{2}(t)\right) .
\end{array}\right.
$$

see Section 8.1 and [48]. As before, we fix the parameters

$$
b=0.9, \quad \varepsilon=0.08, \quad c=2.0528, \quad d=-3.2135, \quad \tau=1.7722,
$$

and take for $f: \mathbb{R} \rightarrow \mathbb{R}$ the sigmoid amplification function $f(u)=\tanh (u)$. The parameters $(\beta, \alpha)$ are used to unfold the singularity.

\footnotetext{
*Department of Mathematics, Hasselt University, Diepenbeek Campus, Agoralaan Gebouw D, 3590 Diepenbeek, Belgium (maikel.bosschaert@uhasselt.be).

${ }^{\dagger}$ Department of Mathematics, Utrecht University, Budapestlaan 6, 3508 TA Utrecht, The Netherlands (s.g.janssens@uu.nl, sj@dydx.nl).

${ }^{\ddagger}$ Department of Mathematics, Utrecht University, Budapestlaan 6, 3508 TA Utrecht, The Netherlands and Department of Applied Mathematics, University of Twente, Zilverling Building, 7500AE Enschede, The Netherlands (I.A.Kouznetsov@uu.nl).

${ }^{1}$ http://ddebiftool. sourceforge.net/

${ }^{2}$ http://pydelay.sourceforge.net/
} 
Remark 23. This demonstration can be found in the directory demos/tutorial/VII/FHN relative to the main directory of the DDE-BifTool package.

\section{S1.1 Generate system files}

Before we start to analyze the system with DDE-BifTool, we first create a system file. This file contains the definition of the system (S1), the standard derivatives needed for calculation of the eigenvalues and eigenvectors, the continuation of bifurcation points and cycles, and also the multilinear forms, see Section 7, used for the calculation of the coefficients of the critical and parameter-dependent normal forms. Alternatively, one can only supply the system itself, see Listing S2. Then finite difference is used to approximate the derivatives. However, this is less efficient and accurate, and therefore not recommended. A separate script gen_sym_FHN.m is used to create a system file. The most important parts of this script are listed and discussed below.

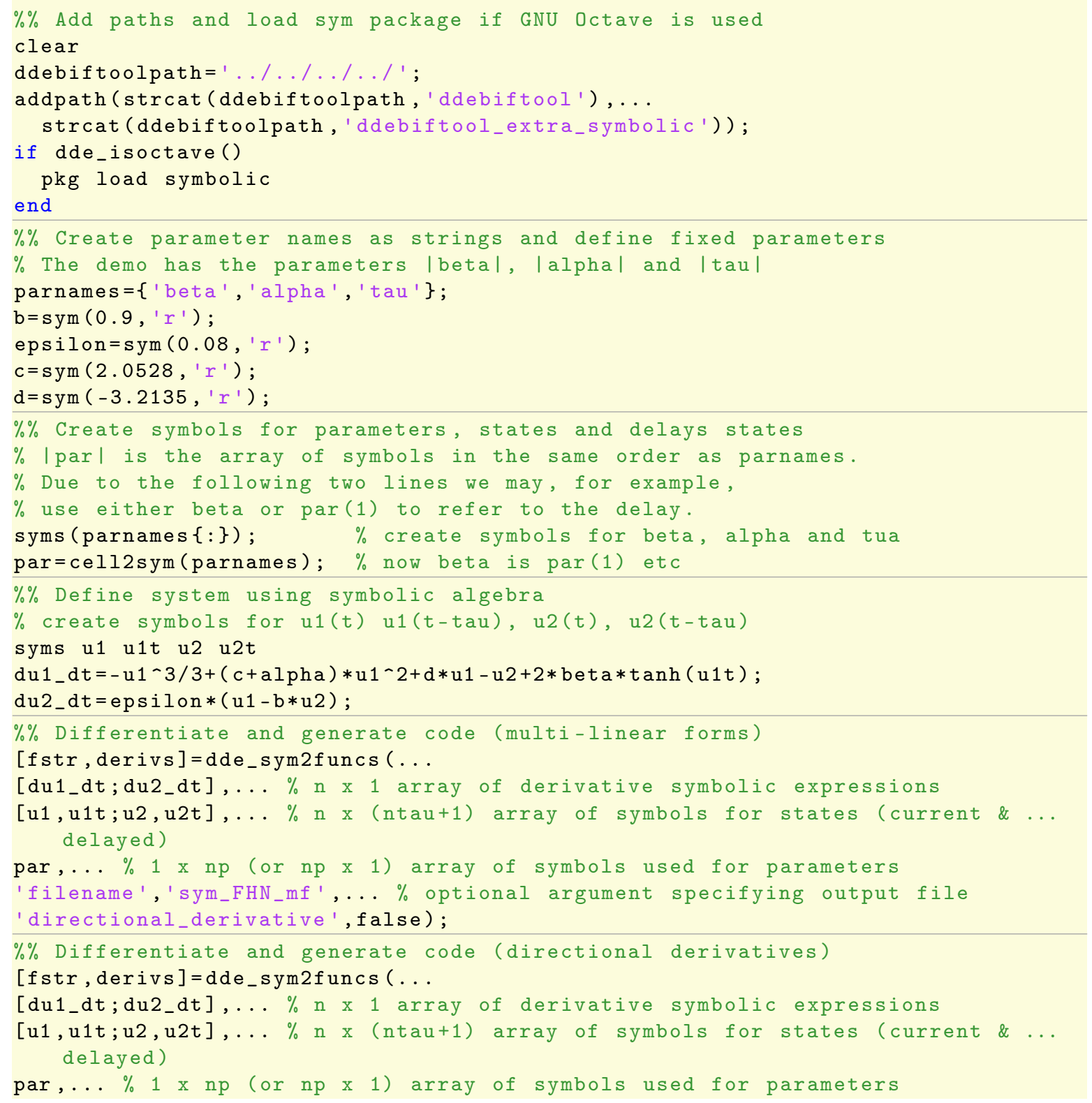




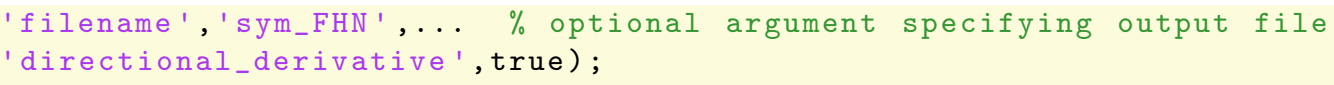

The variable ddebiftoolpath is directed to the DDE-BifTool main folder, which should have been extracted somewhere on the computer. Here a path relative to the current working directory is used. Note that although we only use the parameters $(\beta, \alpha)$ as unfolding parameters, in the current version of DDE-BifTool, we also need to include the delay(s) in the list of parameters. After running the script, the function dde_sym2funcs creates two system files sym_FHN_mf.m and sym_FHN.m. The first file sym_FHN_mf.m implements the higher order derivatives as multilinear forms, as explained in Section 7 , and therefore the file we will solely be using. The second file sym_FHN.m uses directional derivatives to implement the higher order derivatives. The directional derivatives approach formally allows the use of state-dependent delays, see [55]. Although both approaches yields (up to rounding errors) identical normal form coefficients, multilinear forms are much faster.

\section{S1.2 Loading the DDE-BifTool package}

Now that a system file is created we continue with DDE-BifTool to analyze (S1). The code in the following sections highlights the import parts of the file FHN.m. DDE-BifTool consists of a set of MATLAB routines. Thus, to start using DDE-BifTool, we only need to add DDE-BifTool directories to the search path.

Listing S1: Add DDE-BifTool scripts to the search path

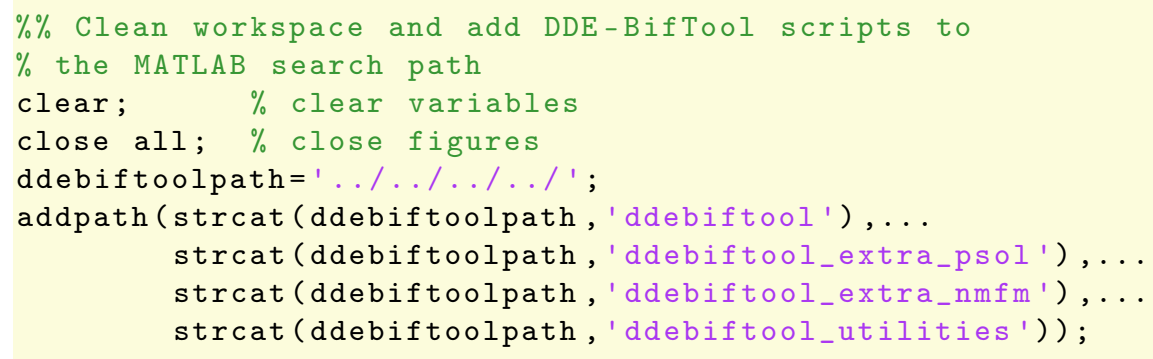

There are four subdirectories added to the search path:

ddebiftool Containing the core files of DDE-BifTool.

ddebiftool_extra_psol An extension for enabling continuation of periodic orbit bifurcations for delay-jifferential equations with constant or state-dependent delay.

ddebiftool_extra_nmfm An extension for normal form computation.

ddebiftool_utilities Containing various utilities.

\section{S1.3 Set parameter names}

The following code allows us to use ind.beta instead of remembering the index of the parameter $\beta$ in the parameter array, and similarly for the other parameters.

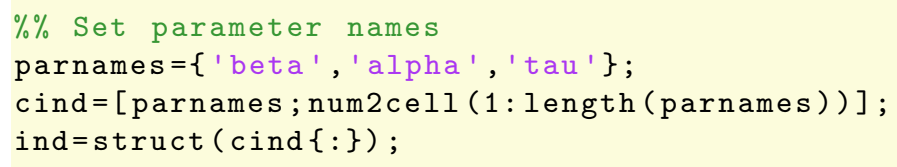

In this way, fewer mistakes are likely to be made and the code is easier to read. 


\section{S1.4 Initialization}

Next, we set up the funcs structure, containing information about where the system and its derivatives are stored, a function pointing to which parameters are delays, and various other settings.

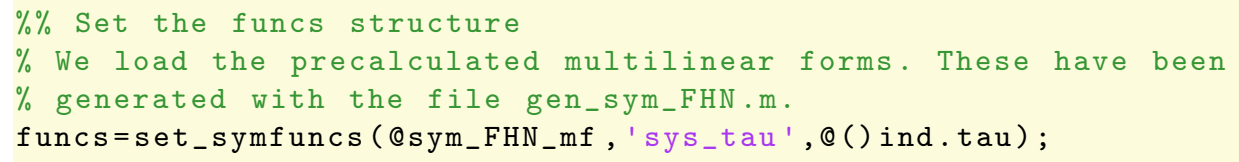

Alternatively, when no system files have been generated, one could initialize the system (S1) as follows.

Listing S2: Define system without a system file

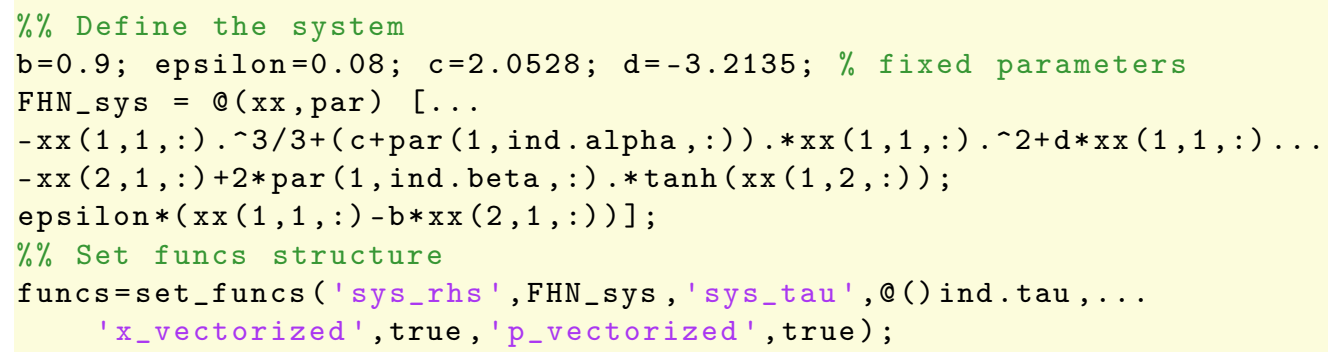

Inspecting the output of the funcs handle gives.

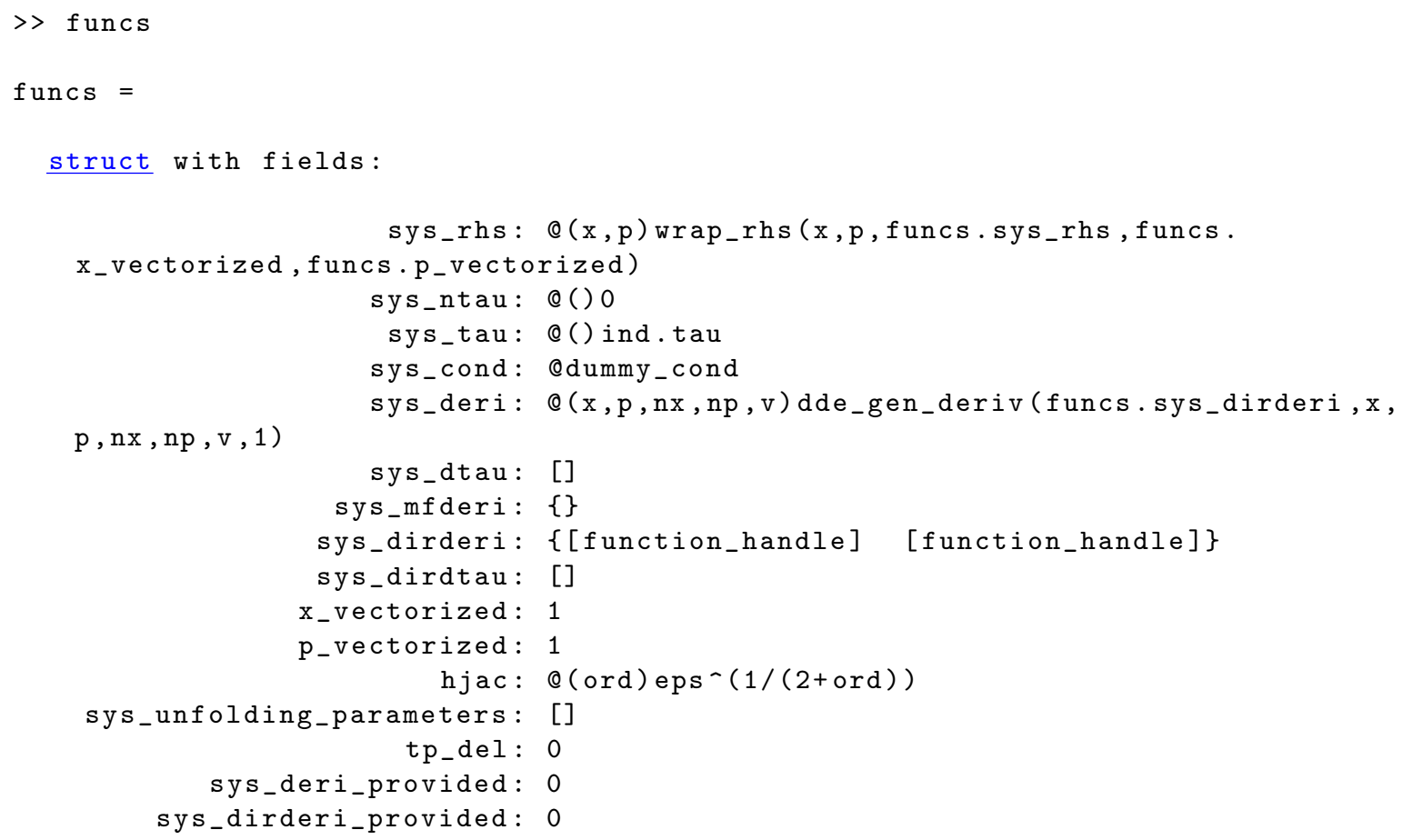

The output shows that no derivative file is supplied. In this case, the derivatives are calculated using finite-difference approximations with the function dde_dirderiv. Again, we do not recommend using the latter approach. However, it can be useful for debugging purposes.

\section{S1.5 Stability and normal form coefficients of the generalized-Hopf point}

We manually specify a steady-state at the generalized-Hopf point found in [48] and calculate its stability. 


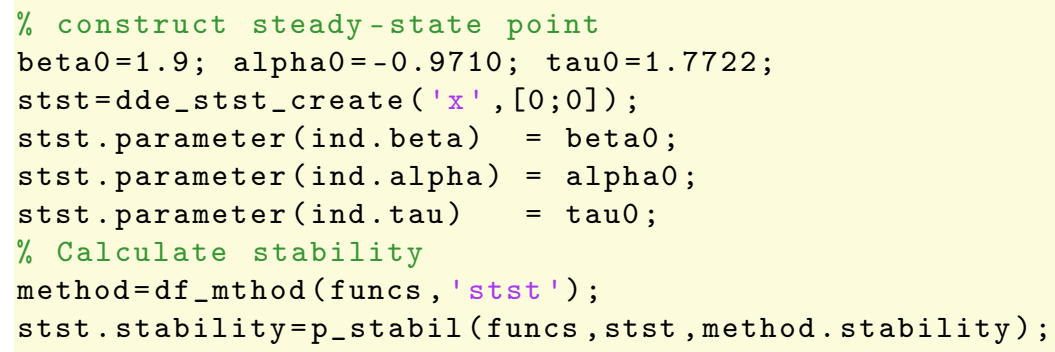

Inspecting the stst.stability structure yields

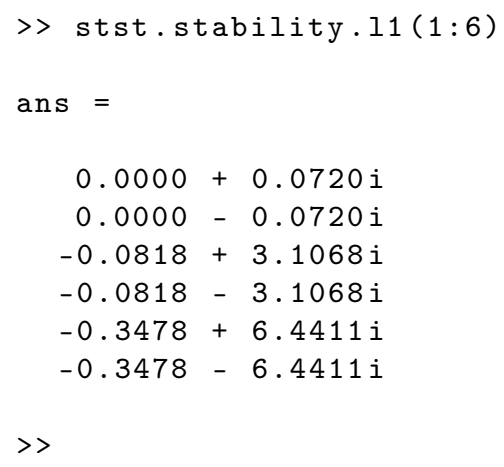

The eigenvalues confirm that the point under consideration is indeed a Hopf point. Next, we convert the steady-state point to a Hopf point and calculate the normal form coefficients with the function nmfm_genh, which implements the coefficients derived in Section 6.1.

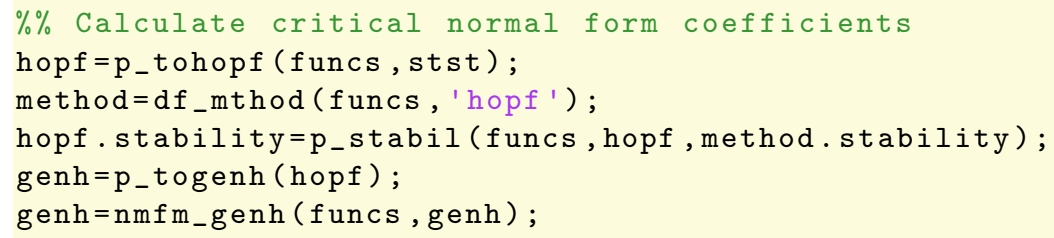

The normal form coefficients are stored in the genh.nmfm structure.

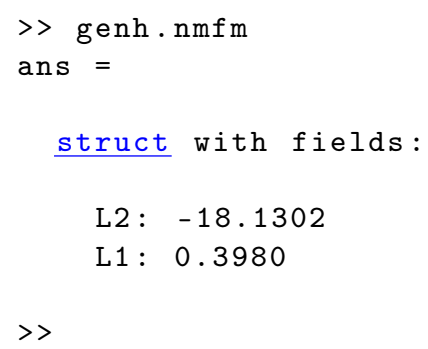

Clearly, the first Lyapunov coefficient (L1) is nonzero. It follows that the Hopf point is not degenerate.

\section{S1.6 Continue Hopf point}

Since the simulations in [48] do indicate a generalized-Hopf point nearby, we continue the Hopf point.

$\%$ Initialize Hopf branch

unfolding_pars=[ind.beta, ind.alpha];

hbr=df_brnch (funcs, unfolding_pars, 'hopf '); 


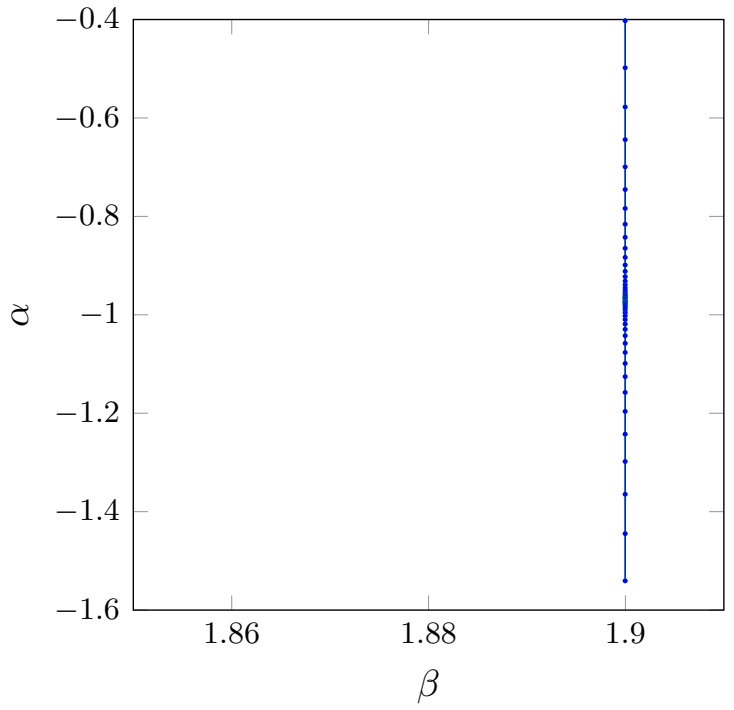

(a) Hopf curve

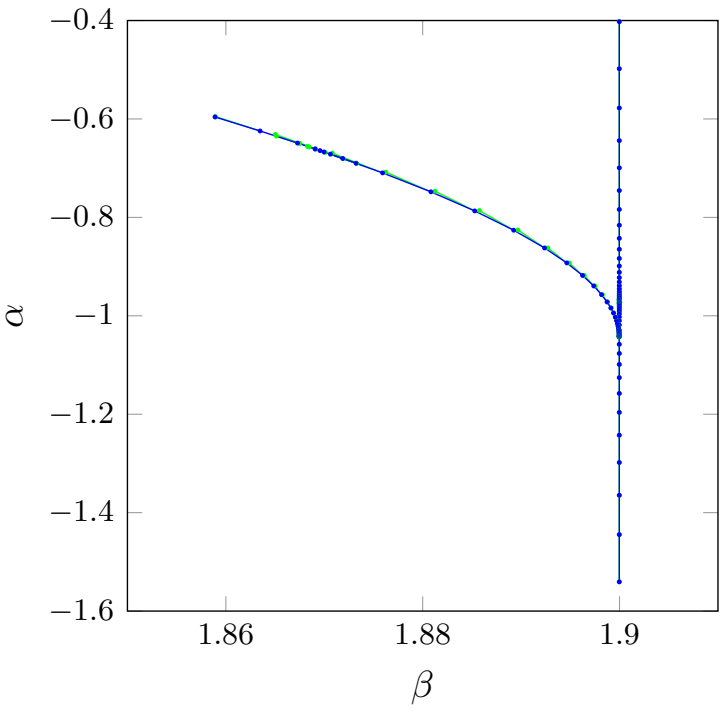

(b) Limit point of cycles curve

Figure S1: (a) Hopf curve continued from the manually constructed point. (b) LPC curve continued from the detected generalized Hopf point using our predictors.

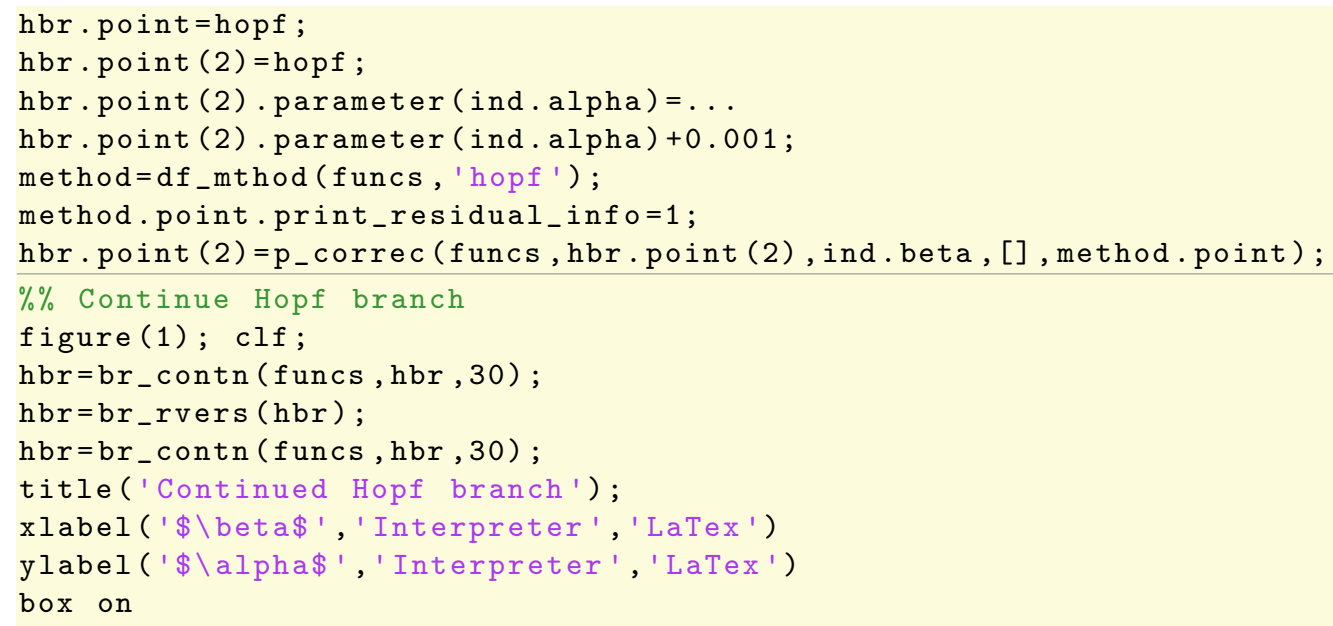

The continued branch hbr is shown in Figure S1a.

\section{S1.7 Detect bifurcation points}

To detect bifurcation points on the Hopf branch, we use the function LocateSpecialPoints.

[hbr_wbifs, hopftests, hc2_indices, hc2_types]=... LocatespecialPoints (funcs, hbr);

The MATLAB console shows the following output.

HopfCodimension2: calculate stability if not yet present HopfCodimension2: calculate L1 coefficients 


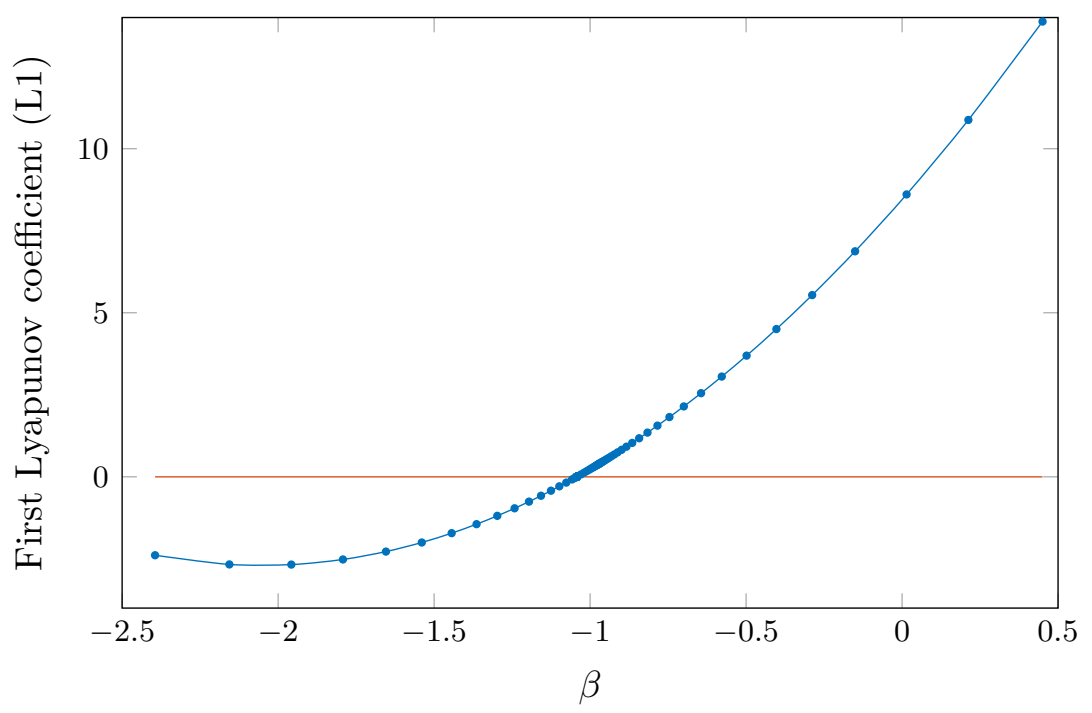

Figure S2: Plot of the test function for a generalized Hopf point.

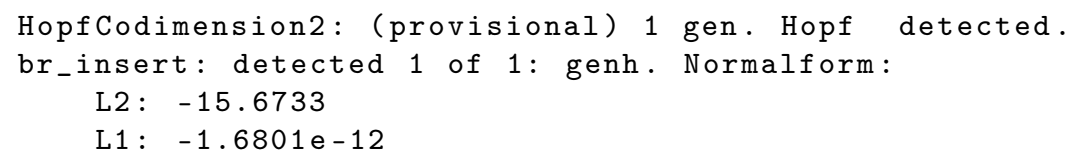

Thus a generalized Hopf point is indeed present on the Hopf branch hbr. The returned branch hbr_wbifs contains this point. The array hc2_indices is used to subtract the generalized Hopf point below. If there would be more bifurcation points detected, hc2_types can be used to inspect their types. Lastly, hopftests stores the test functions to detect a bifurcation point. A change in sign in one of these functions indicates a bifurcation. The code below plots the test function for the generalized Hopf point, i.e. the first Lyapunov coefficient (L1), see Figure S2.

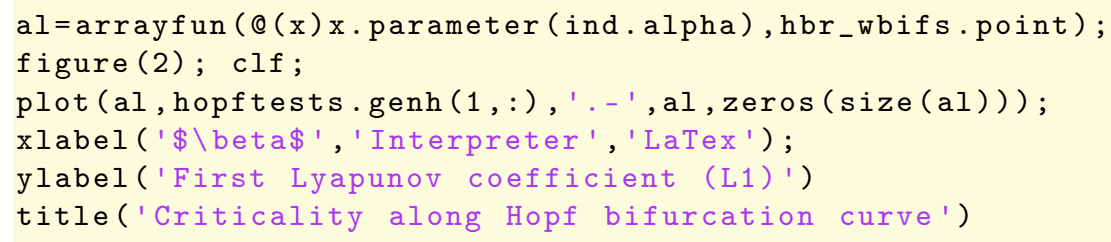

\section{S1.8 Continue limit point of cycle curve}

First, we subtract the detected generalized-Hopf point from the branch.

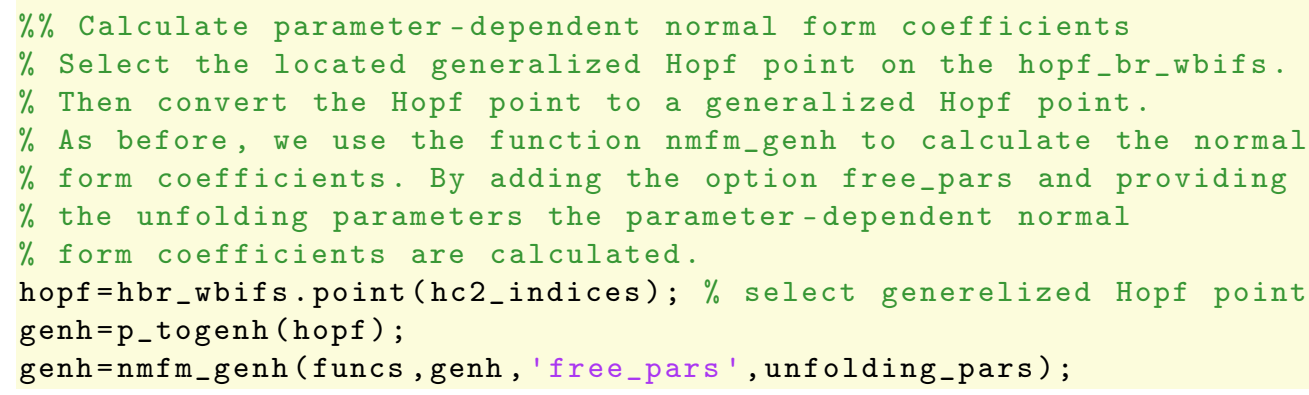


genh. nmfm. L1

By inspecting the genh structure, we obtain the correct parameter values of the generalized Hopf bifurcation.

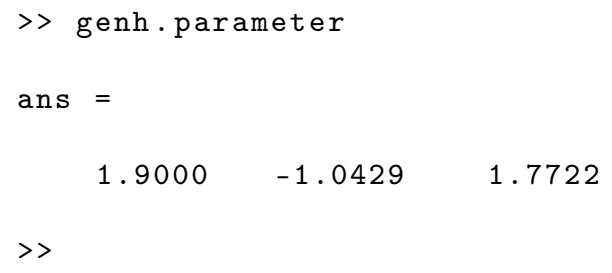

To continue the limit point of cycles curve emanating from the generalized Hopf point, we use the function C1branch_from_C2point.

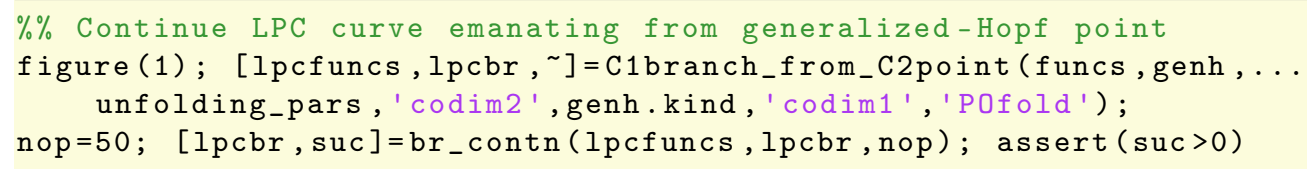

This function uses the file nmfm_POfold_from_genh_init.m in which the predictor, see Sections 5.1 and 6.1.3, is implemented. Using this function, a branch with three initial corrected cycles is created which is continued in the standard way, see Figure S1b.

\section{S1.9 Calculate predicted periodic orbits}

To compare the computed parameter values and periodic orbits on the branch lpcbr with the predictor, we again use the function C1branch_from_C2point, but with the additional argument predictor set to 1 and step to an interval of $\varepsilon$-values. Now the cycles are left uncorrected.

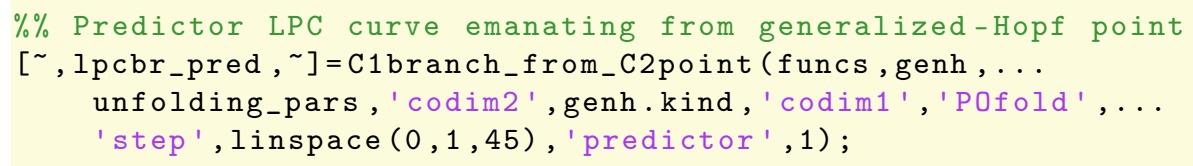

\section{S1.10 Bifurcation diagram}

The following code produces the bifurcation diagram presented in the main text, see Figure 1, and has been reproduced here in Figure S3. The figure was exported with the MATLAB and GNU Octave compatible package matlab2tikz, see [26].

Listing S3: MATLAB code for bifurcation diagram

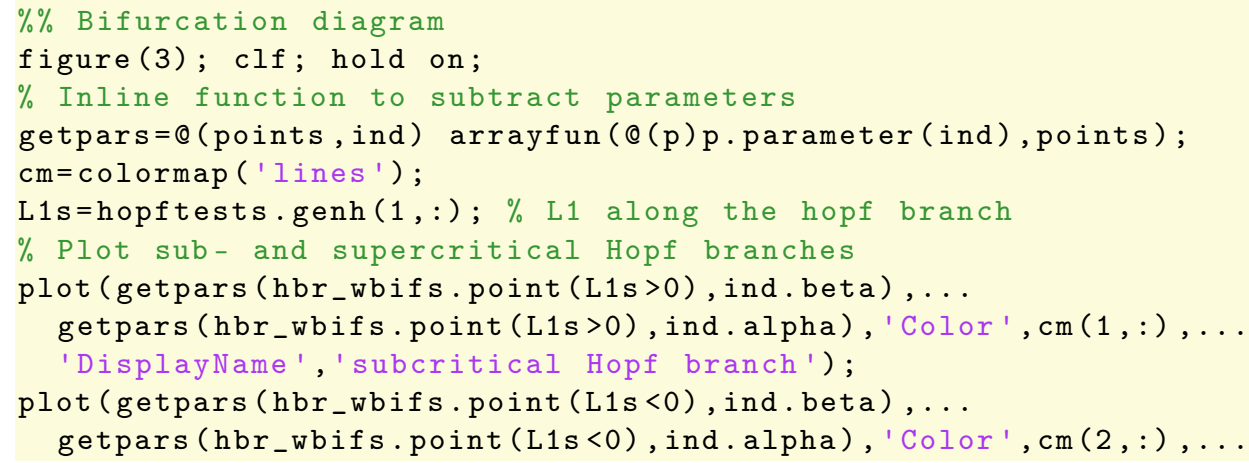



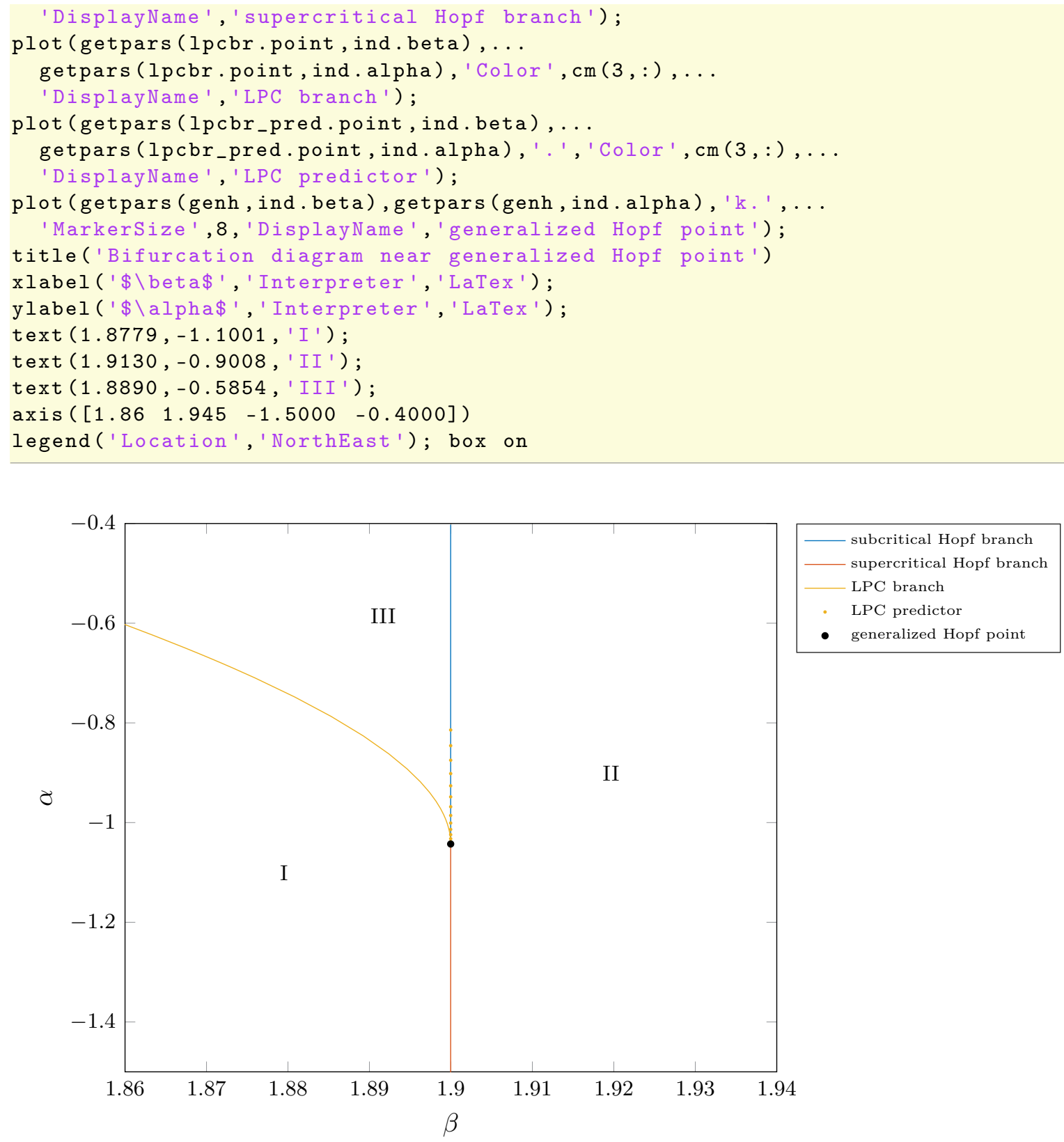

Figure S3: Bifurcation diagram near the generalized Hopf point in the system (S1) with unfolding parameters $(\beta, \alpha)$. The bifurcation curves are nearly identical to those in the bifurcation diagram of the topological normal form as presented in [43, page 314].

\section{S1.11 Plot comparing computed and predicted periodic orbits}

Lastly, we create a plot to compare the computed and predicted periodic orbits.

$\%$ Plot comparing computed and predicted periodic orbits

figure(4); clf; hold on; 


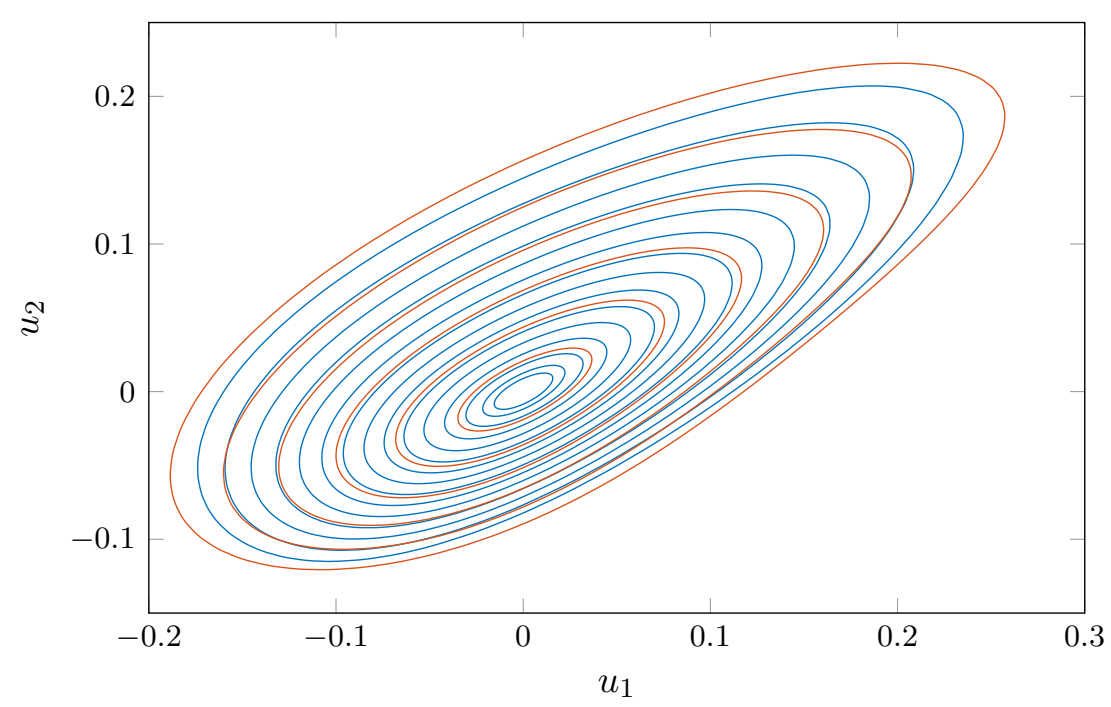

Figure S4: Comparison between computed periodic orbits (blue) and predicted periodic orbits (red) emanating from the generalized Hopf bifurcation.

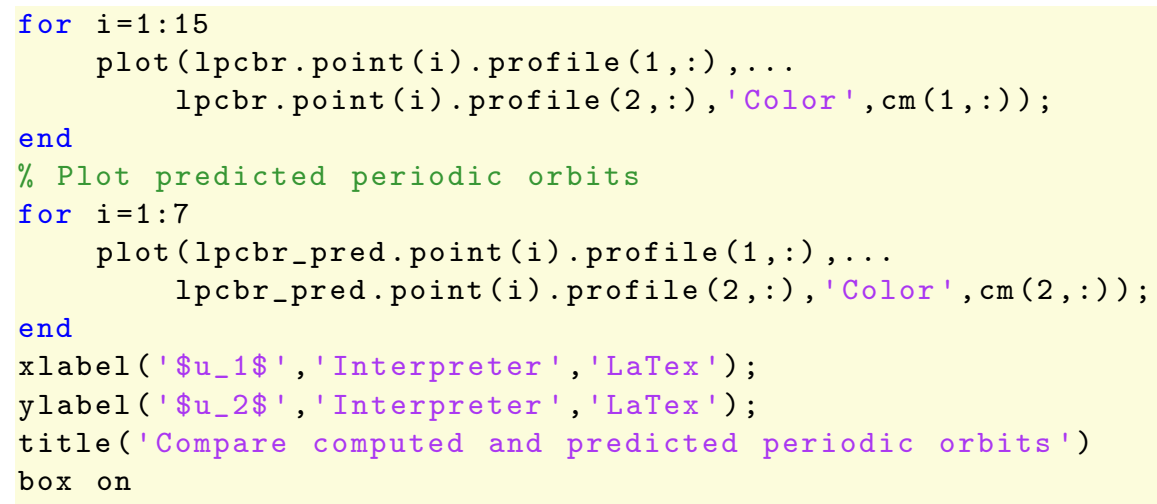

The resulting plot is shown in Figure S4. Note that the cycles shown have different underlying parameter values. Nonetheless, we see that the cycles are in good agreement.

\section{S1.12 Simulation with MATLAB}

Next, we simulate the dynamics near the generalized Hopf point. For this, we take a point in each of the three regions as shown in Figure S3. The following code, from the file FHN_simulation.m, uses the MATLAB function dde23.

$\%$ Clean workspace and add DDE-BifTool scripts

$\%$ to the MATLAB search path.

clear; $\quad \%$ clear variables

close all; \% close figures

ddebiftoolpath=' ../.../../';

addpath (strcat (ddebiftoolpath, 'ddebiftool'),...

strcat (ddebiftoolpath, 'ddebiftool_extra_psol'),...

strcat (ddebiftoolpath, 'ddebiftool_extra_nmfm'),...

strcat (ddebiftoolpath, 'ddebiftool_utilities')) ; 


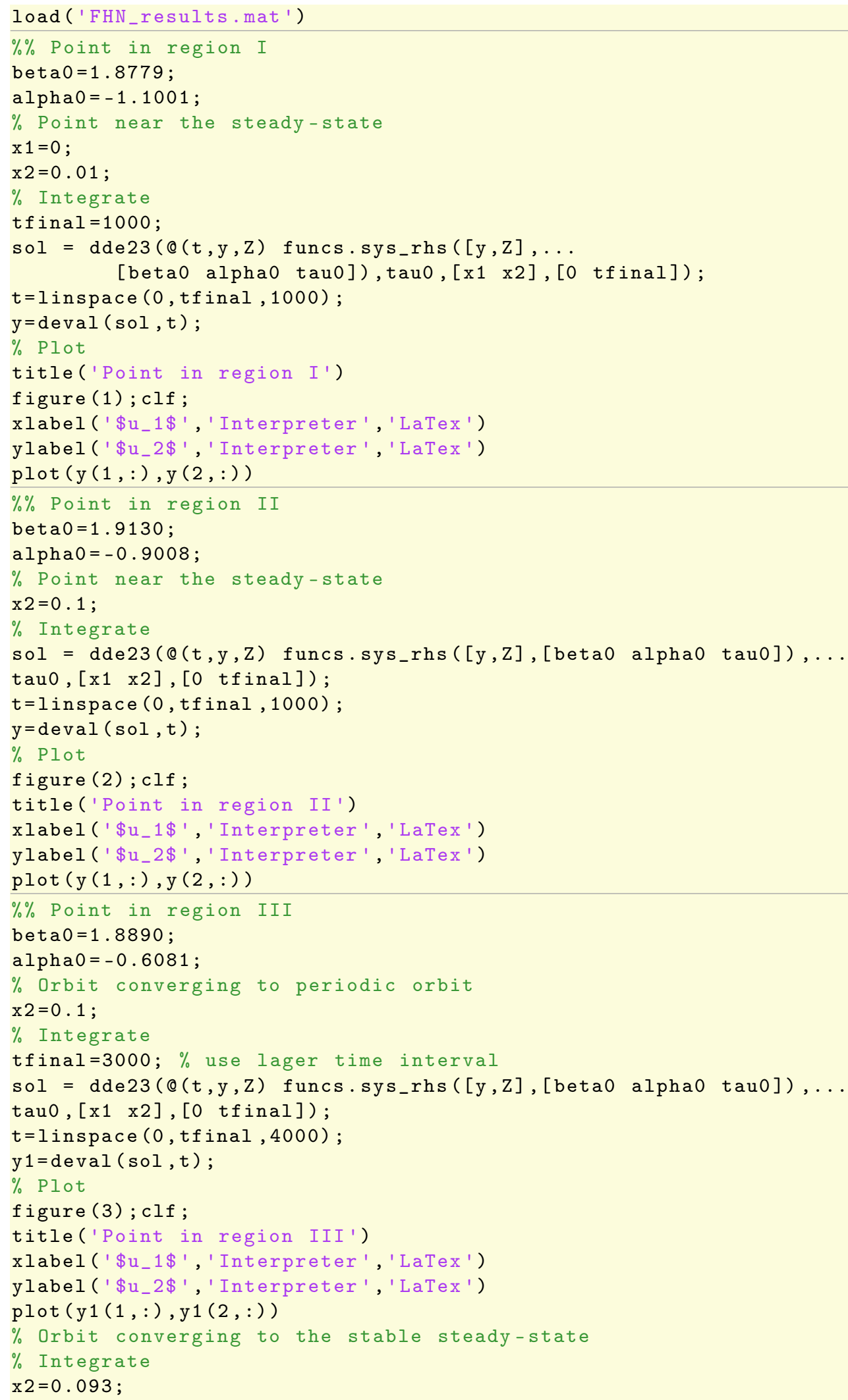




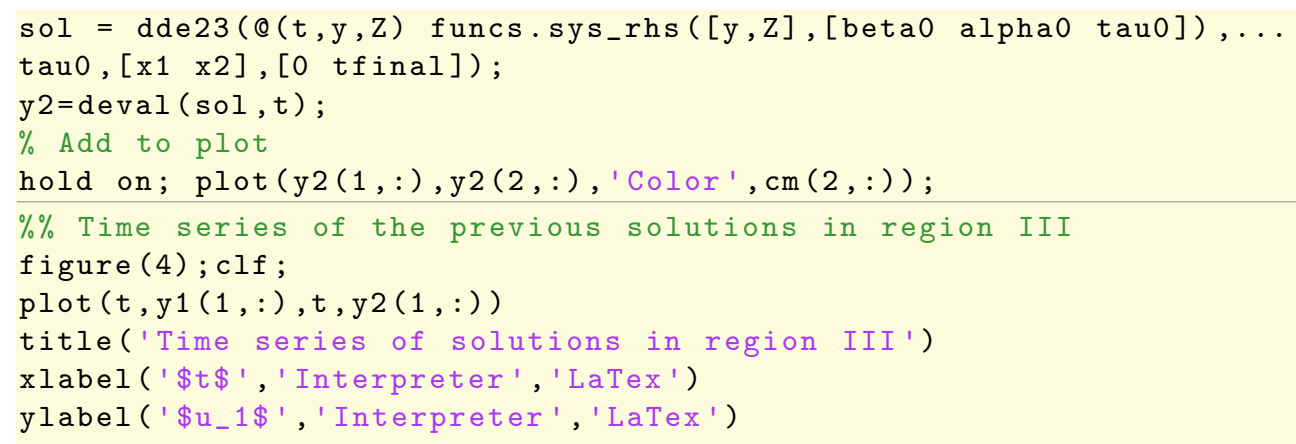

In Figures S5a to S5d the resulting plots are shown, confirming the dynamics near the generalized Hopf point as predicted in [43].

\section{S2 Fold-Hopf bifurcation in the Rose-Hindmarsh model with time delay}

In [48] a Rose-Hindmarsh model [33,34] with time delay in the self-feedback process,

$$
\left\{\begin{array}{l}
\dot{x}(t)=y(t)-a x^{3}(t)+b x^{2}(t-\tau)-c z(t)+I_{a p p}, \\
\dot{y}(t)=c-d x^{2}(t)-y(t) \\
\dot{z}(t)=r(S(x(t)-\chi)-z(t))
\end{array}\right.
$$

is considered, see Section 8.2. The parameters values

$$
a=1.0, \quad b=3.0, \quad c=1.0, \quad d=5.0, \quad \chi=-1.6, \quad r=0.001
$$

are fixed and $\left(I_{a p p}, S\right)$ are the unfolding parameters.

Remark 24. This demonstration can be found in the directory demos/tutorial/VII/RH relative to the main directory of the DDE-BifTool package. Here, we omit the code to generate a system file. The system file sym_RH_mf.m has been generated with the script gen_sym_RS.m. Also, we assume that the DDE-BifTool package has been loaded as in Listing S1. The code in Sections S2.1 to S2.10 highlights the important parts of the file RH.m.

\section{S2.1 Set parameter names and funcs structure}

As in the previous example, we set the parameter names and define the funcs structure.

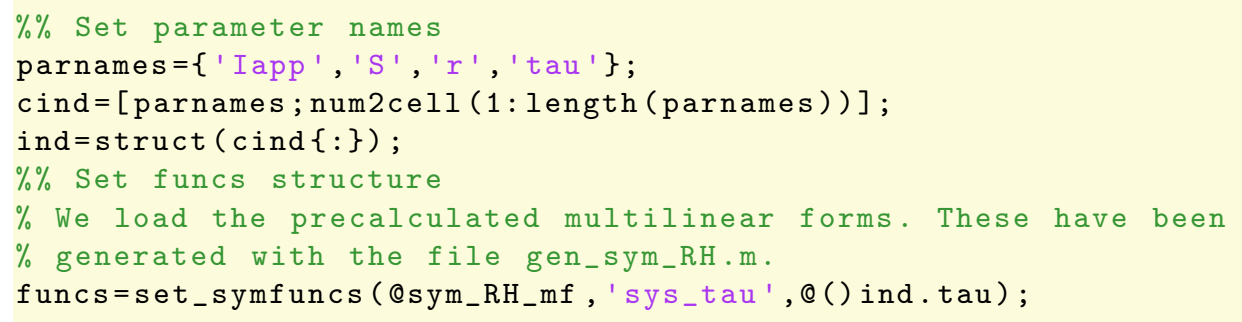

\section{S2.2 Stability and normal form coefficients of the fold-Hopf point}

We construct a steady-state at the fold-Hopf point and calculate its stability. 


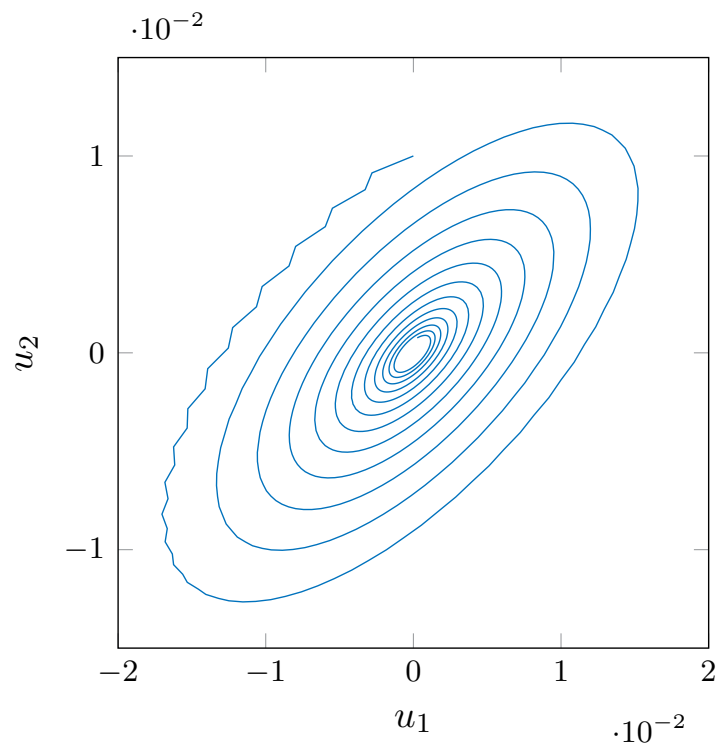

(a)

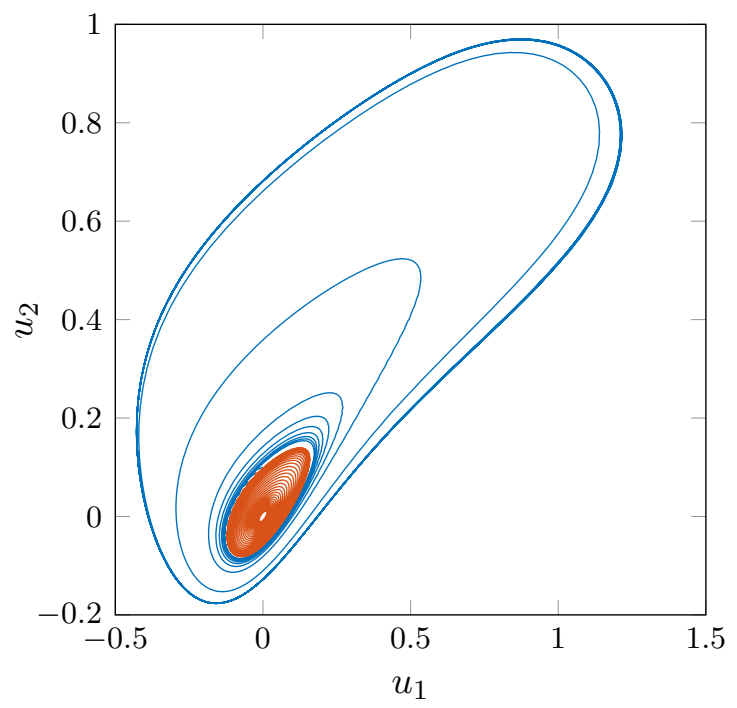

(c)

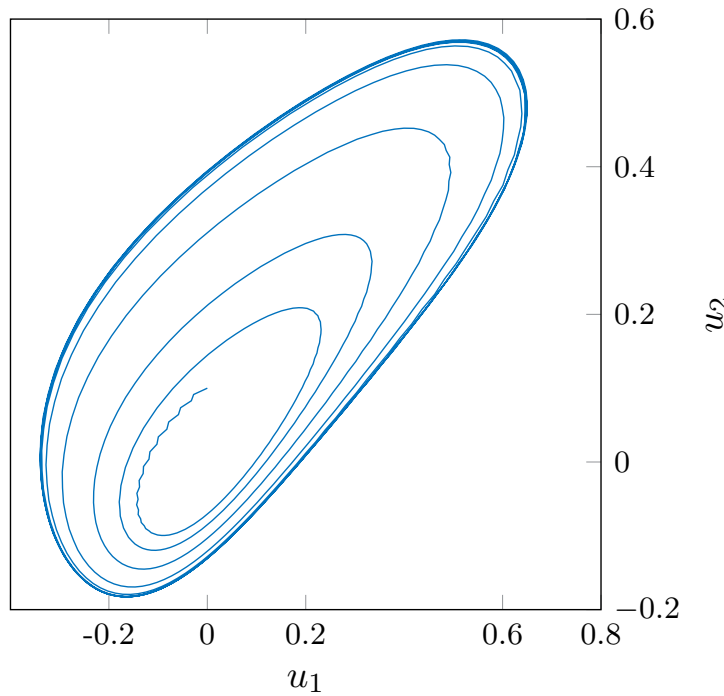

(b)

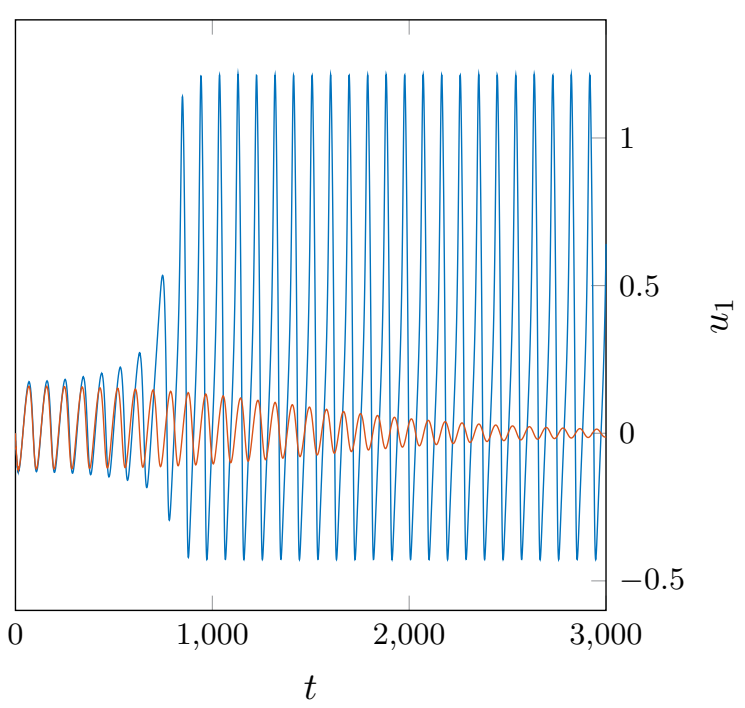

(d)

Figure S5: Simulation near the generalized Hopf point in the system (S1). In (a) we see a stable steadystate corresponding to a point in region I. When we enter region II, the stability of the steady-state is lost and a stable cycle appears, as seen in (b). In region III, there is a stable steady-state inside a stable cycle. This is confirmed in (c) and (d). In (c), the initial point of the orbit in blue is just outside the unstable cycle and converges to the stable cycles. The initial point of the orbit in red is just inside the unstable cycle and converges to the stable steady-state. In (d), the time series of these orbits are shown in the $\left(t, u_{1}(t)\right)$ plane.

$\%$ Construct fold-Hopf point

$\mathrm{a}=1.0 ; \mathrm{b}=3.0 ; \mathrm{c}=1.0 ; \mathrm{d}=5.0 ; \mathrm{chi}=-1.6$;

$r=1.0 e-03$; 


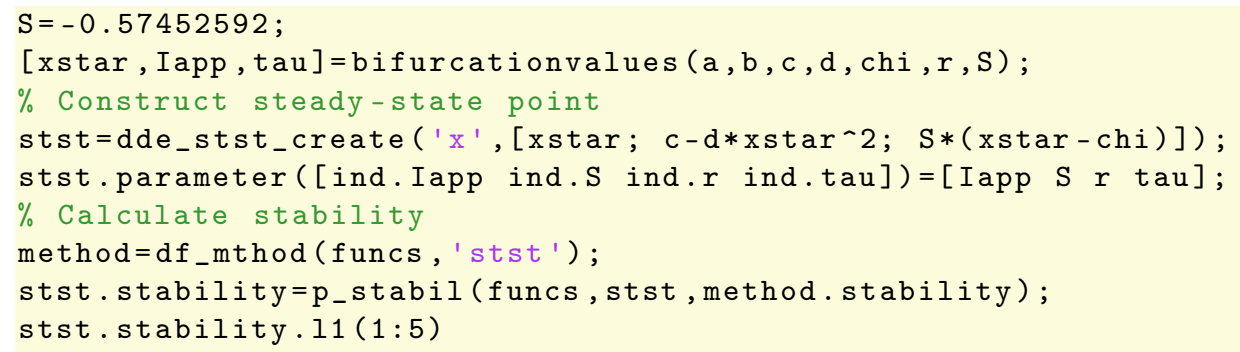

The function bifurcationvalues calculates $\left(x_{\star}, I_{a p p}, \tau\right)$ according to the formulas as given in Section 8.2. The MATLAB console shows the following output.

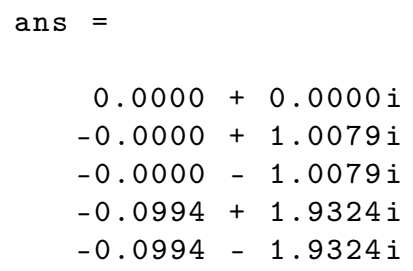

We have a zero eigenvalue and a pair of purely imaginary eigenvalues. Furthermore, the remaining eigenvalues have negative real parts. Next, we calculate the normal form coefficients and the transformation to the center manifold with the function nmfm_zeho, which implements the coefficients as derived in Section 6.2. For this we need to set the argument free_pars to the unfolding parameter $\left(I_{a p p}, S\right)$. These coefficients will be used to start the continuation of the various branches emanating from the fold-Hopf point.

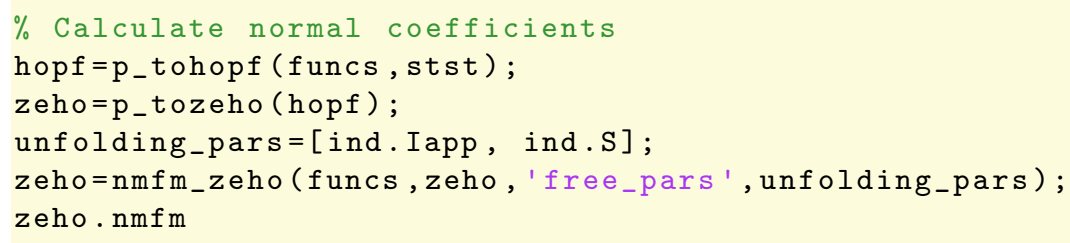

The MATLAB console shows the following output.

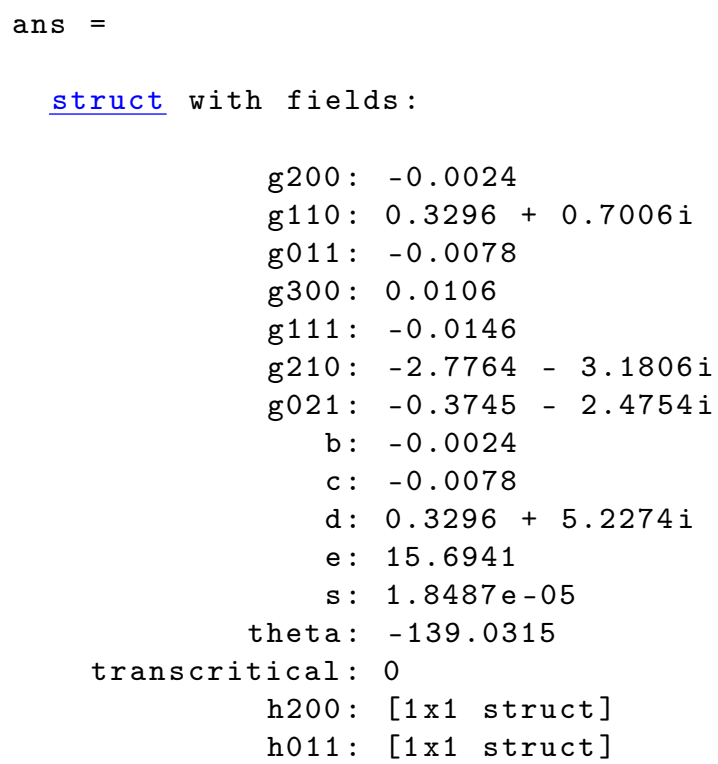




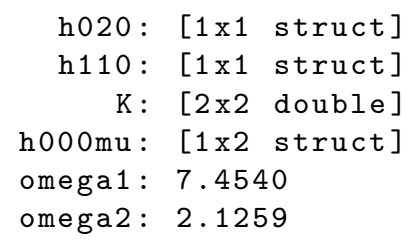

Since $s>0$ and $\theta(0)<0$ global bifurcations or invariant tori are present. However, since the sign of $e$ is positive the stability of the invariant tori will be unstable for nearby parameter values. It follows that the torus observed in [48] by simulations does not originate from the fold-Hopf point under consideration.

\section{S2.3 Set bifurcation parameter range and step size bounds}

Before continuing the various branches emanating from the fold-Hopf point, we create the variable brpars containing parameter bounds and maximal stepsizes.

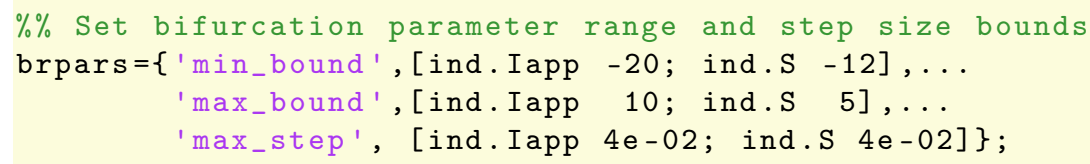

\section{S2.4 Continue NS, Hopf and fold branch}

As in the previous example, we use the function C1branch_from_C2point to start to continue the branches emanating from the fold-Hopf point. Figure S6 is created using similar code as in Listing S3. We remark that even when there would be stable tori present for nearby parameter values, the window in which these tori would exist is quite small. Indeed, the parameter values would have to be below the Hopf curve and above the Neimark-Sacker curve to the left of the fold-Hopf point.

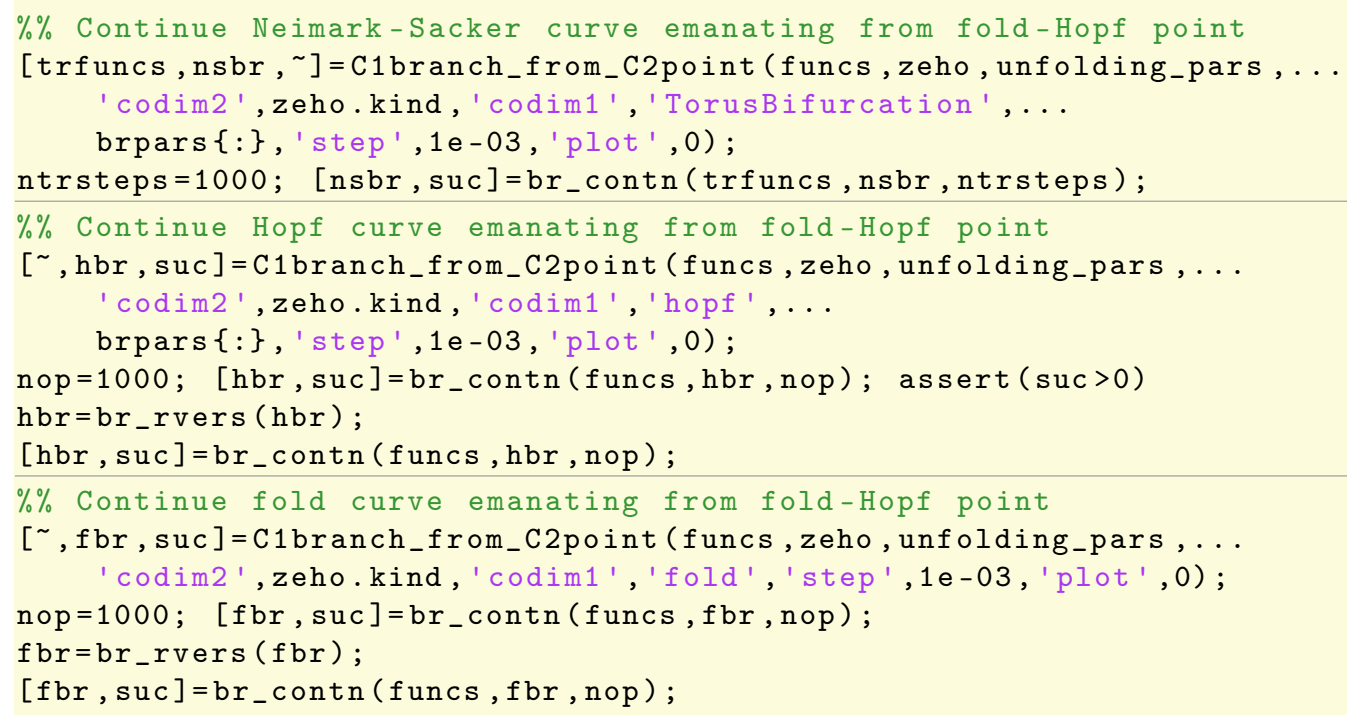

\section{S2.5 Stable invariant tori}

We change the parameters $r=1.4$ and $S=-8$, while keeping the other fixed parameters as in (S3). Using the formulas given in Section 8.2, we calculate $x_{\star}, I_{a p p}$ and $\tau$. 


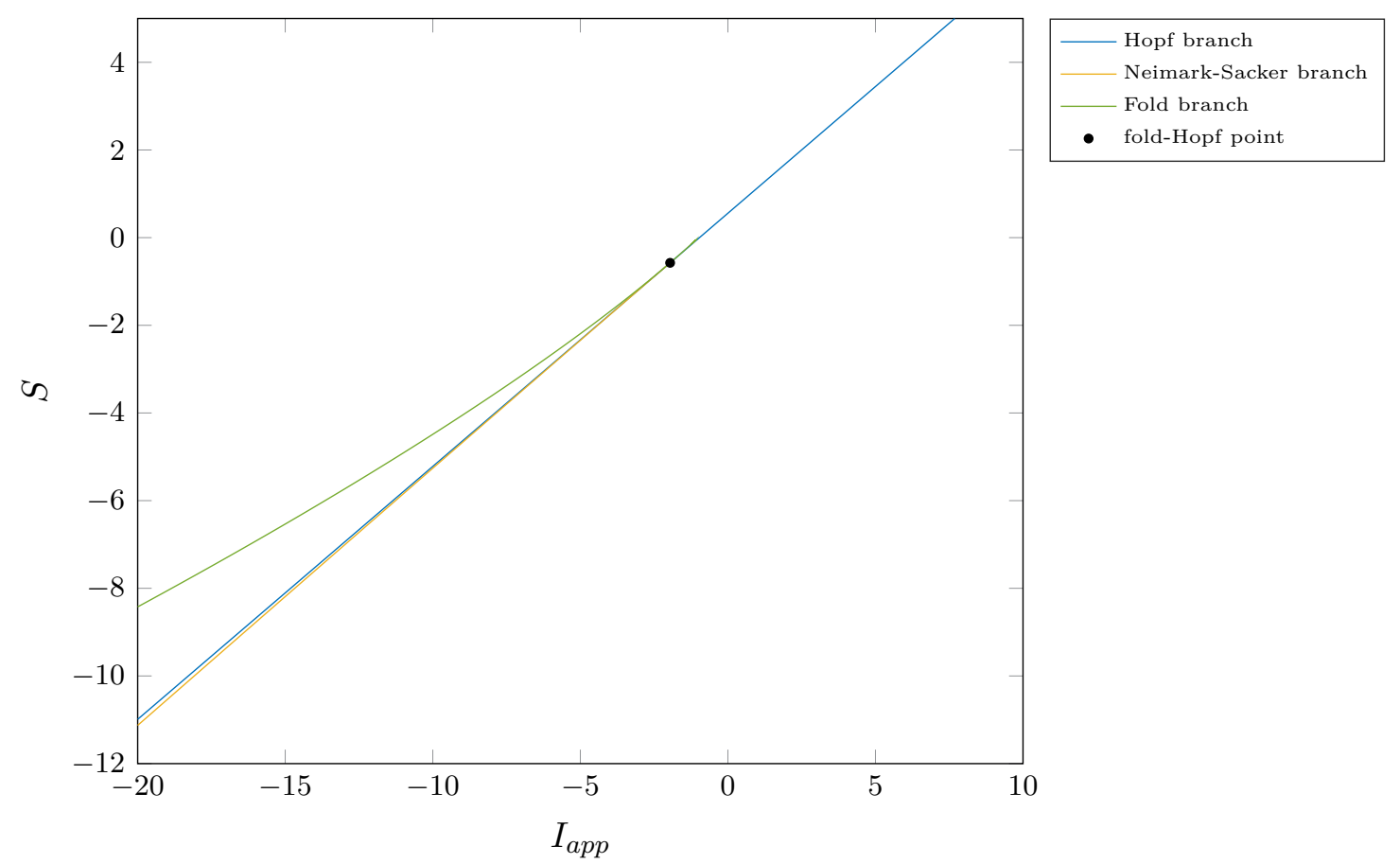

Figure S6: Bifurcation diagram near the fold-Hopf point in (S2) with $(r, S)=(0.001,-0.57452592)$.

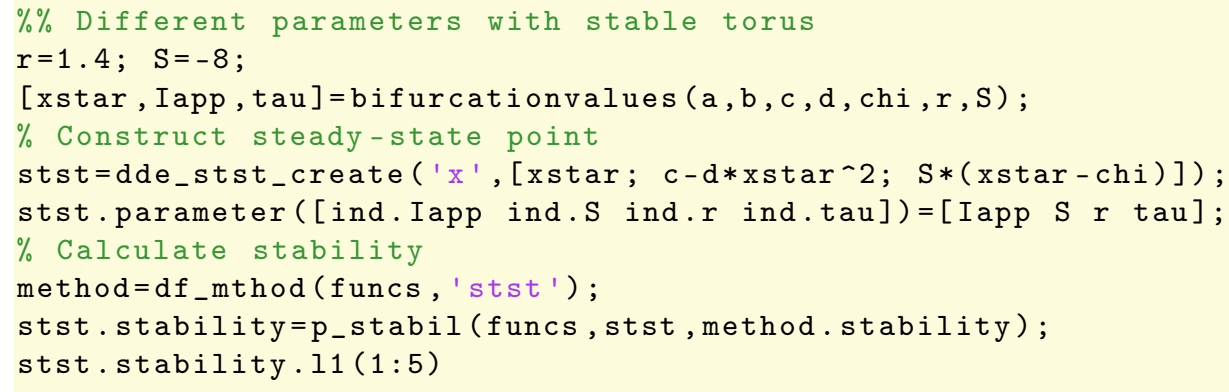

The MATLAB console outputs

ans $=$

$$
\begin{array}{r}
0.00000+0.00000 i \\
0.00000+5.60424 i \\
0.00000-5.60424 i \\
-0.27870+0.00000 i \\
-0.66607+11.94839 i \\
-0.66607-11.94839 i
\end{array}
$$

which are indeed the eigenvalues that should be present at a fold-Hopf bifurcation. Next, we calculate the normal form coefficients.

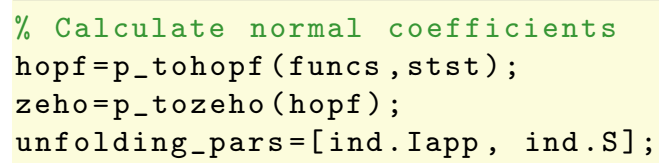


zeho=nmfm_zeho(funcs, zeho, 'free_pars ', unfolding_pars);

Inspecting the normal form coefficients yields

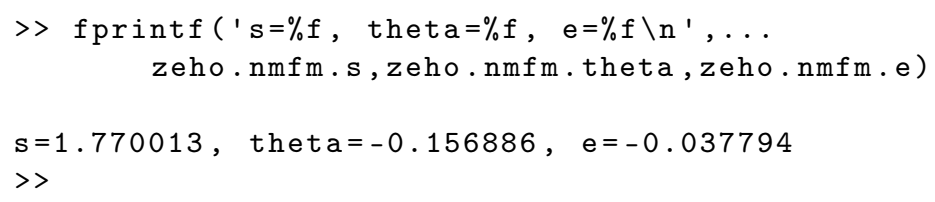

The coefficients $s$ and $\theta(0)$ reveal that we are in case III of the fold-Hopf bifurcation, see [43, page 342]. Since the sign of $e(0)$ is negative, there is a time reversal to take into account. Therefore, we expect stable tori to be present for nearby parameter values.

\section{S2.6 Adjusting bifurcation parameter range}

We adjust the variable brpars to reflect the current situation.

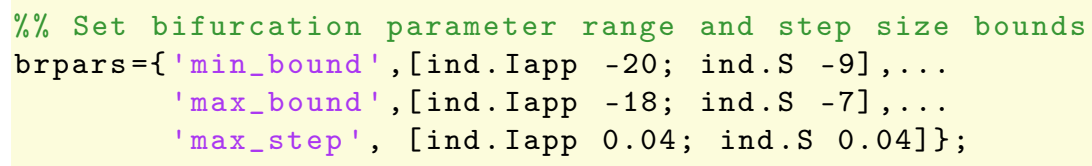

\section{S2.7 Detect special points on the Hopf branch}

Since the code to continue the Neimark-Sacker, Hopf and fold curves is identical to the code in Section S2.4, we continue with detecting bifurcations on the Hopf branch. The Hopf points on the branch hbr_wbifs will contain the normal form coefficients L1 and L2. These will be used to visualize the criticality of the Hopf points (sub or super) in the bifurcation diagram.

[hbr_wbifs, hopftests, hc2_indices, hc2_types]=.. .

LocatespecialPoints (funcs, hbr);

\section{S2.8 Predictors}

As in the previous example, we obtain predictors for the various branches simply by setting the argument predictor to 1 and the argument step to a range of $\varepsilon$-values when calling the function C1branch_from_C2point.

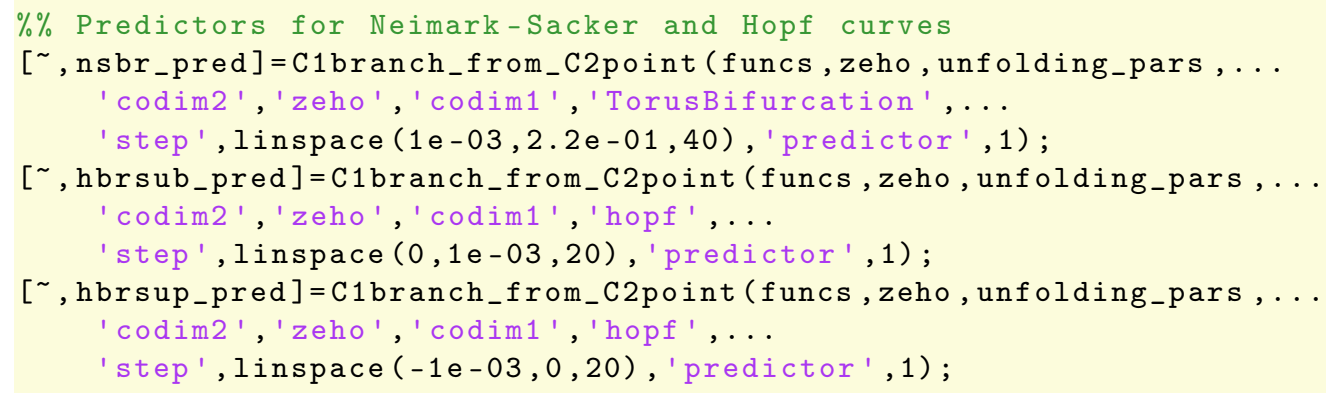




\section{S2.9 Bifurcation diagram}

We plot the obtained curves and the predictor for the Neimark-Sacker and Hopf curve with the following code.

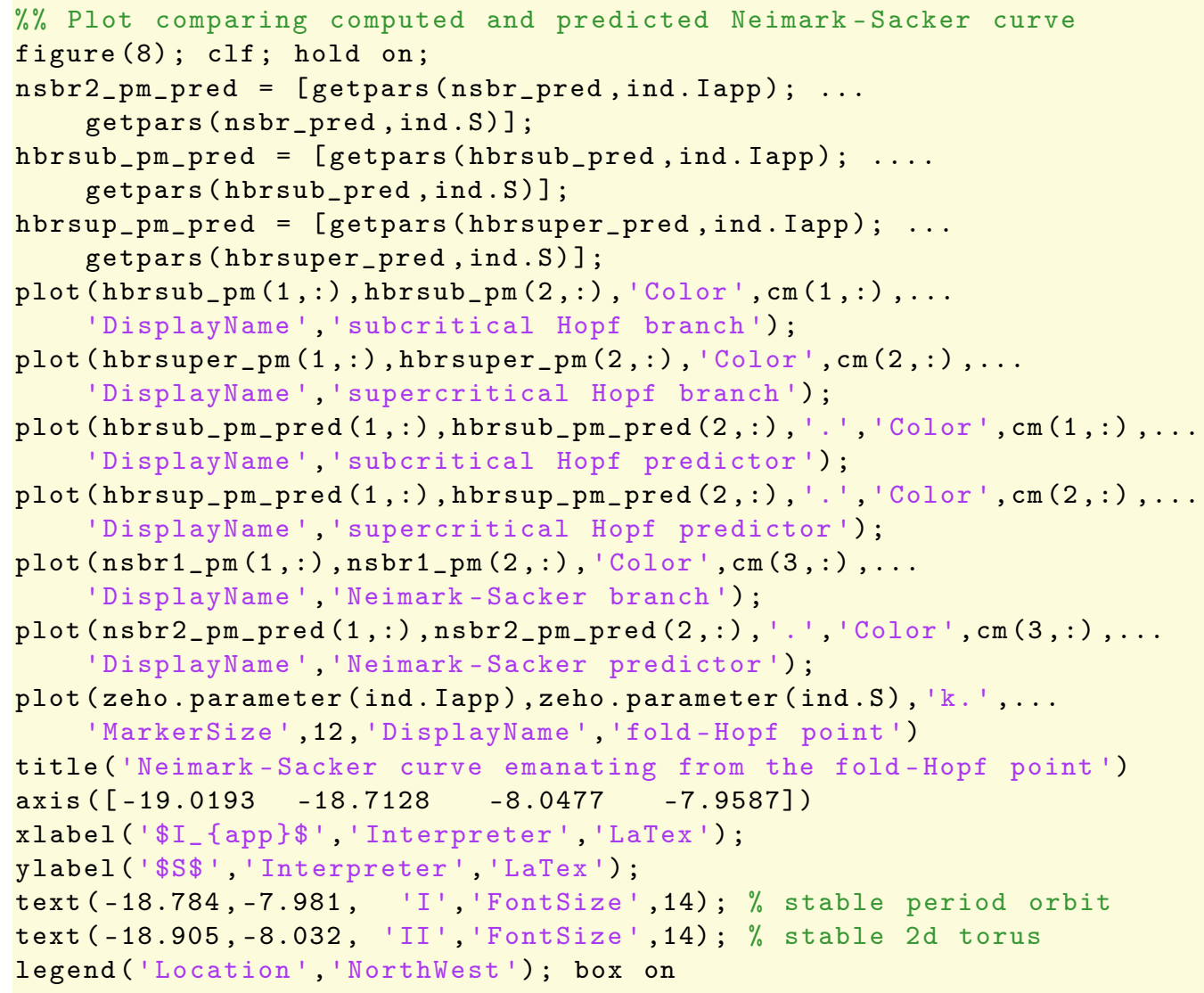

Figure S7 shows the resulting bifurcation diagram.

\section{S2.10 Plots comparing computed and predicted periodic orbits}

We create a plot to compare the computed and predicted periodic orbits.

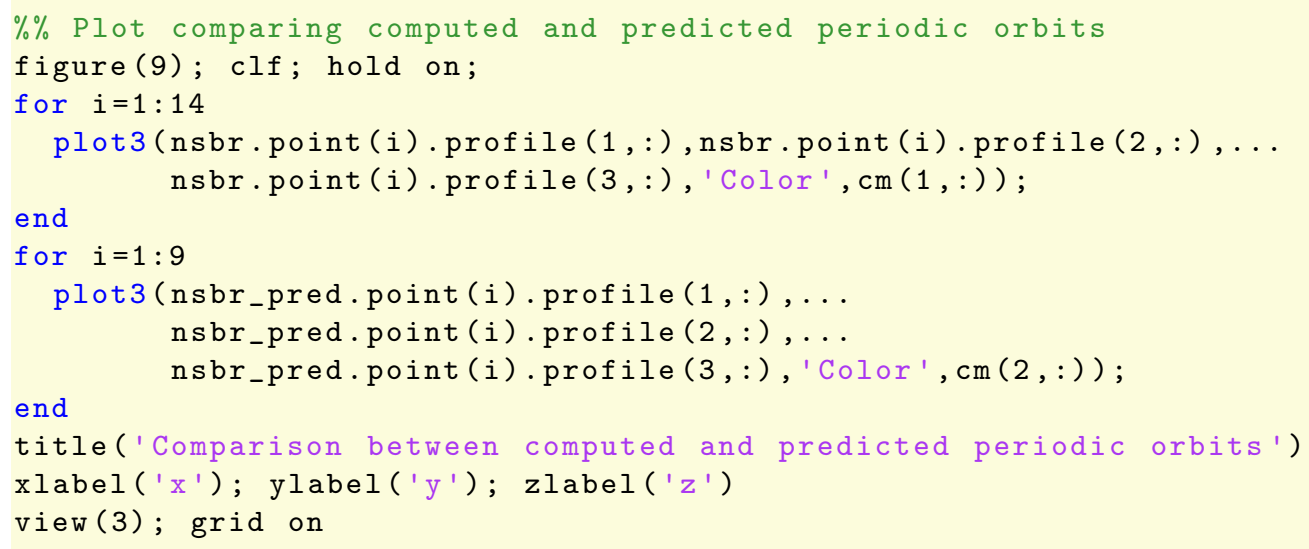




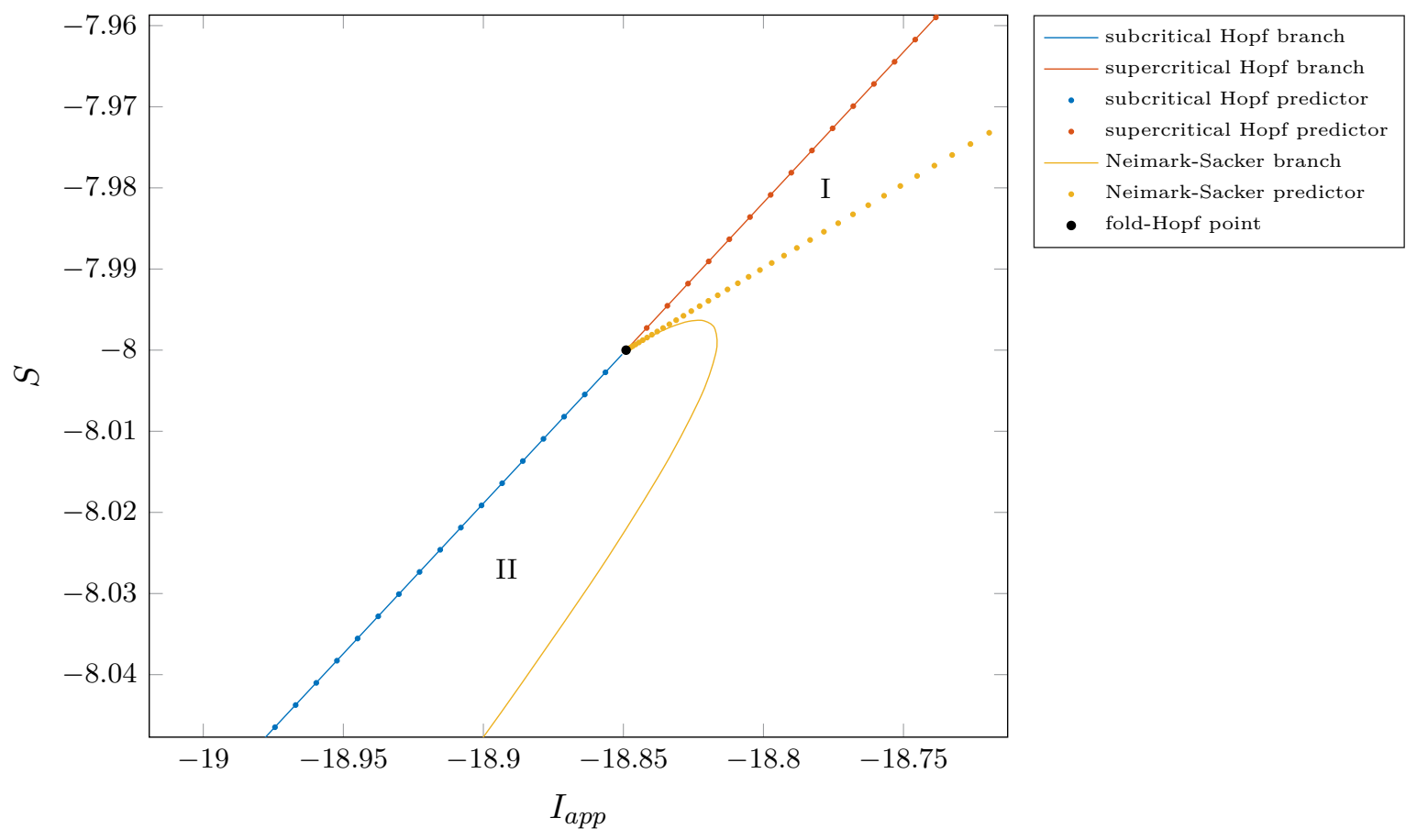

Figure S7: Bifurcation diagram near the fold-Hopf point in (S2) with $(r, S)=(1.4,-8)$. The fold branch is not included here since it is indistinguishable from the Hopf curve at this scale.

The resulting plot is shown in Figure S8a. To compare the computed and predicted periods, we use the following code.

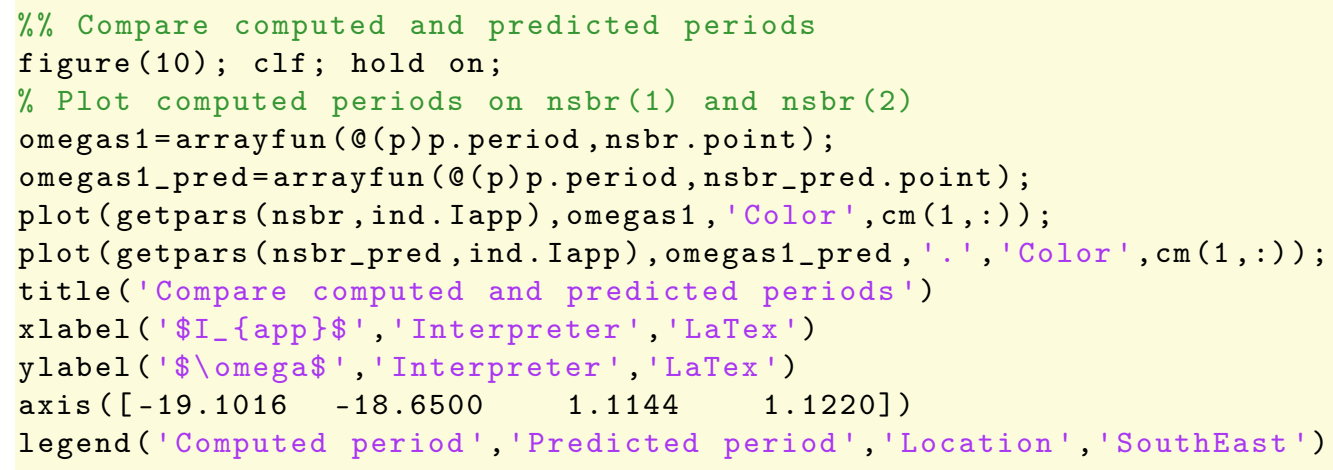

The resulting plot is shown in Figure S8b.

\section{S2.11 Simulation with pydelay}

In this section we simulate the dynamics near the fold-Hopf point. The following code, from the file RH_simulation_torus.py, uses the Python package pydelay [24].

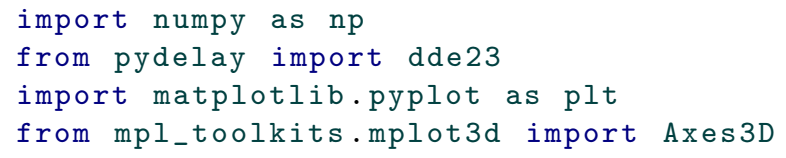




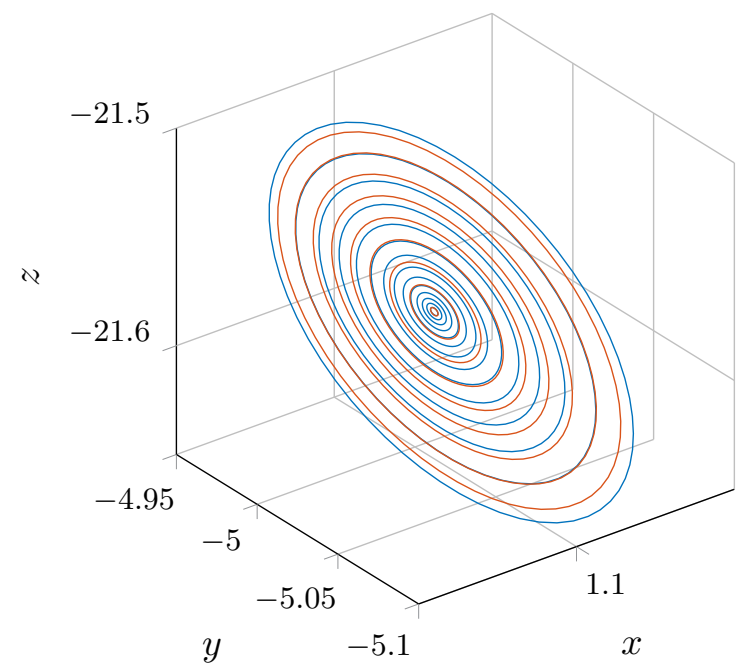

(a)

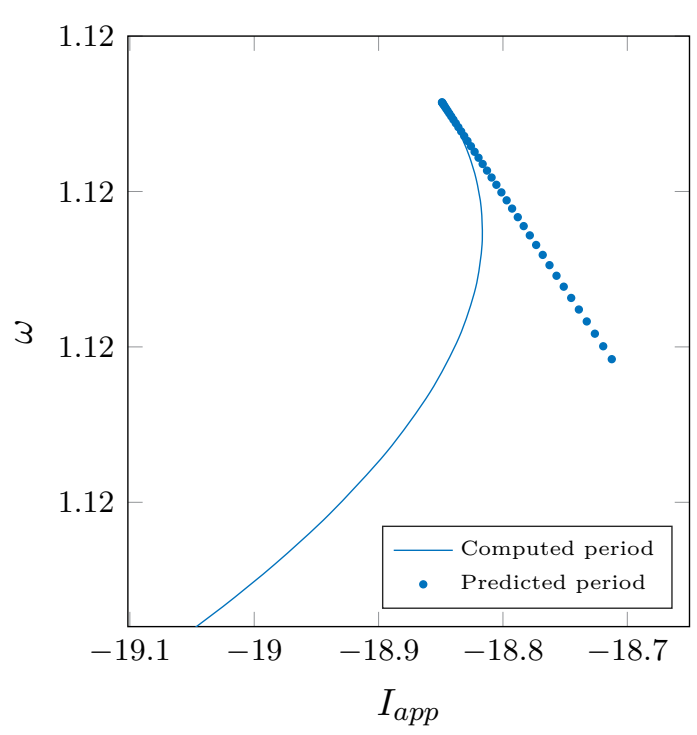

(b)

Figure S8: In (a) the computed periodic orbits (blue) and predicted periodic orbits (red) are compared. In (b) the computed predicted periods of the cycles are compared. We see that both are in good agreement.

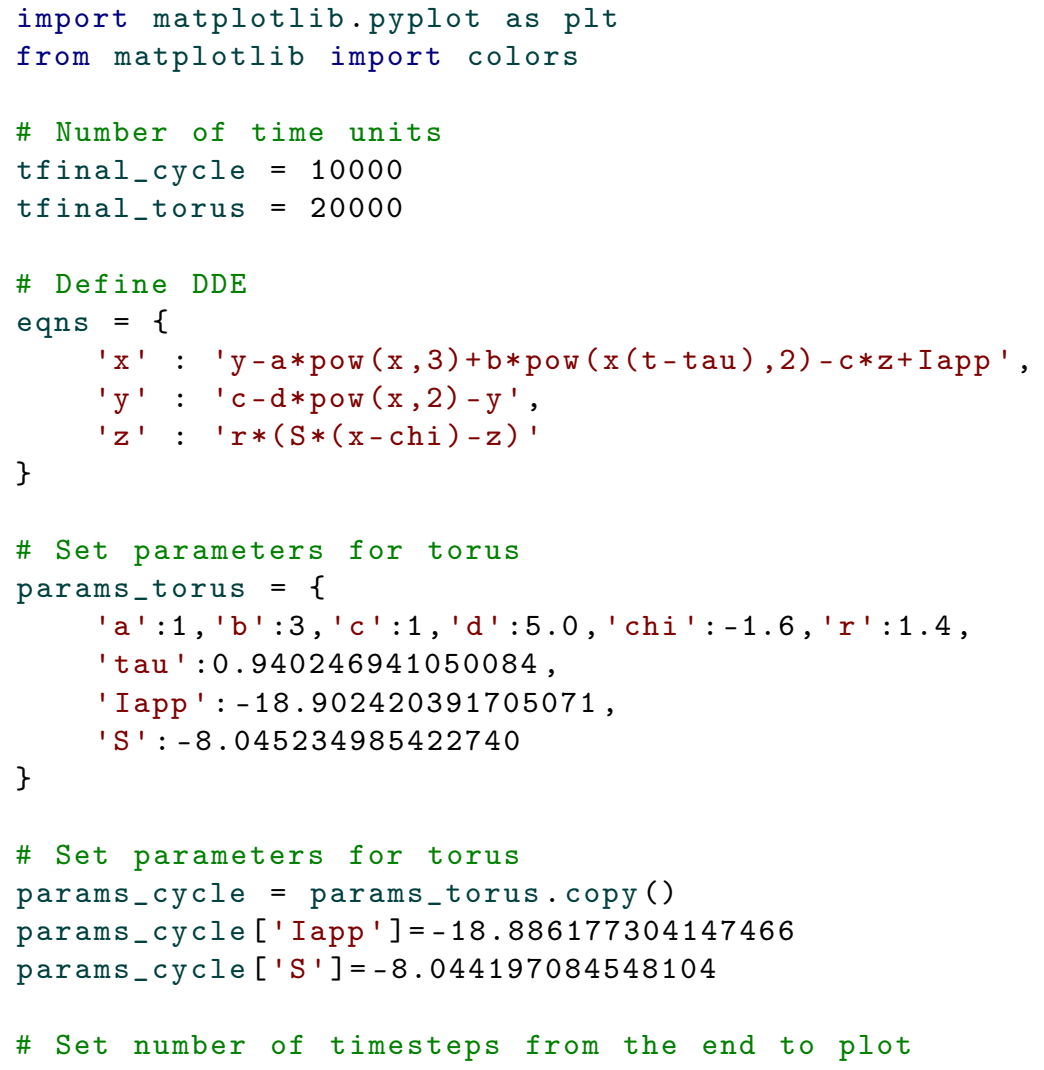




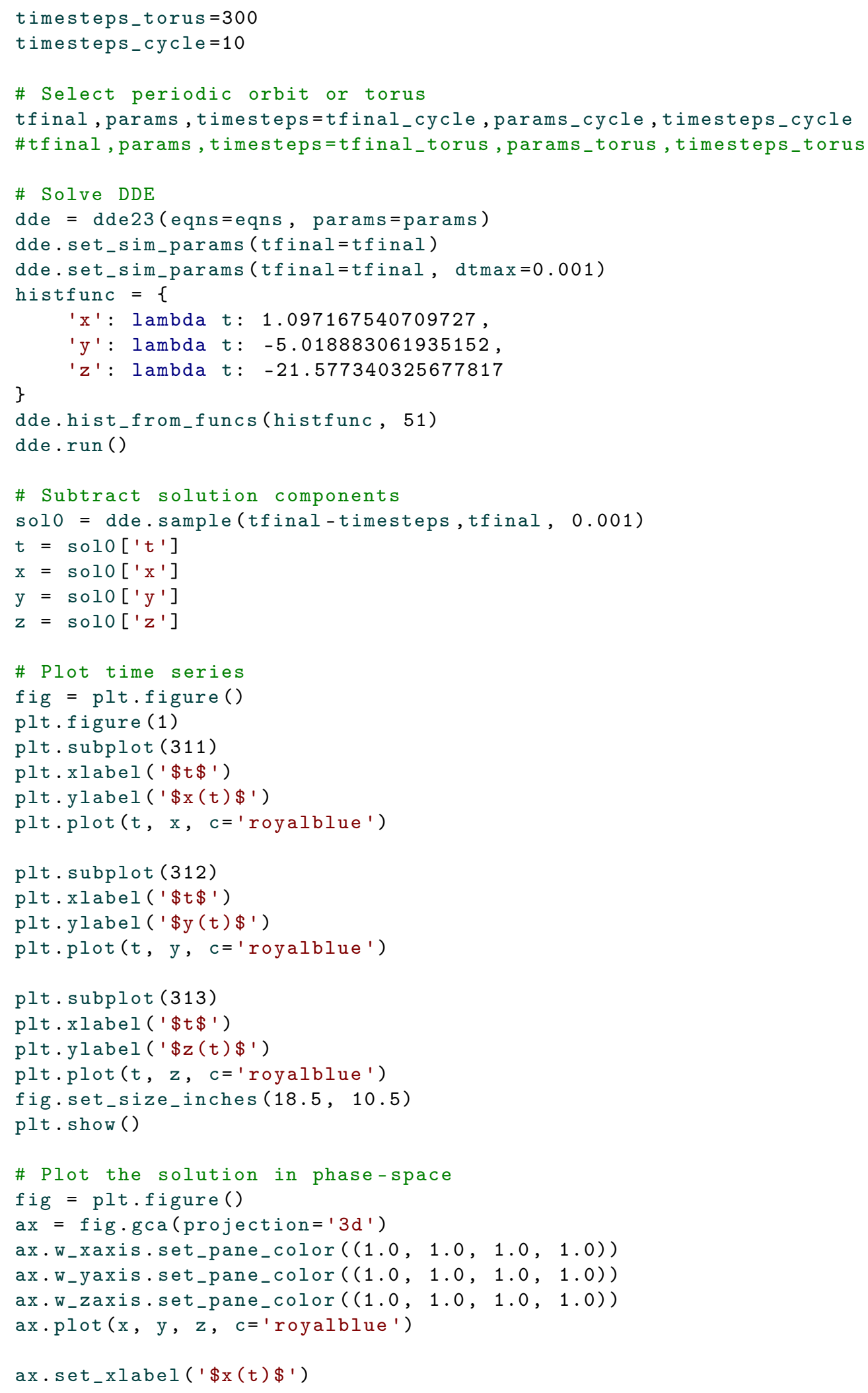




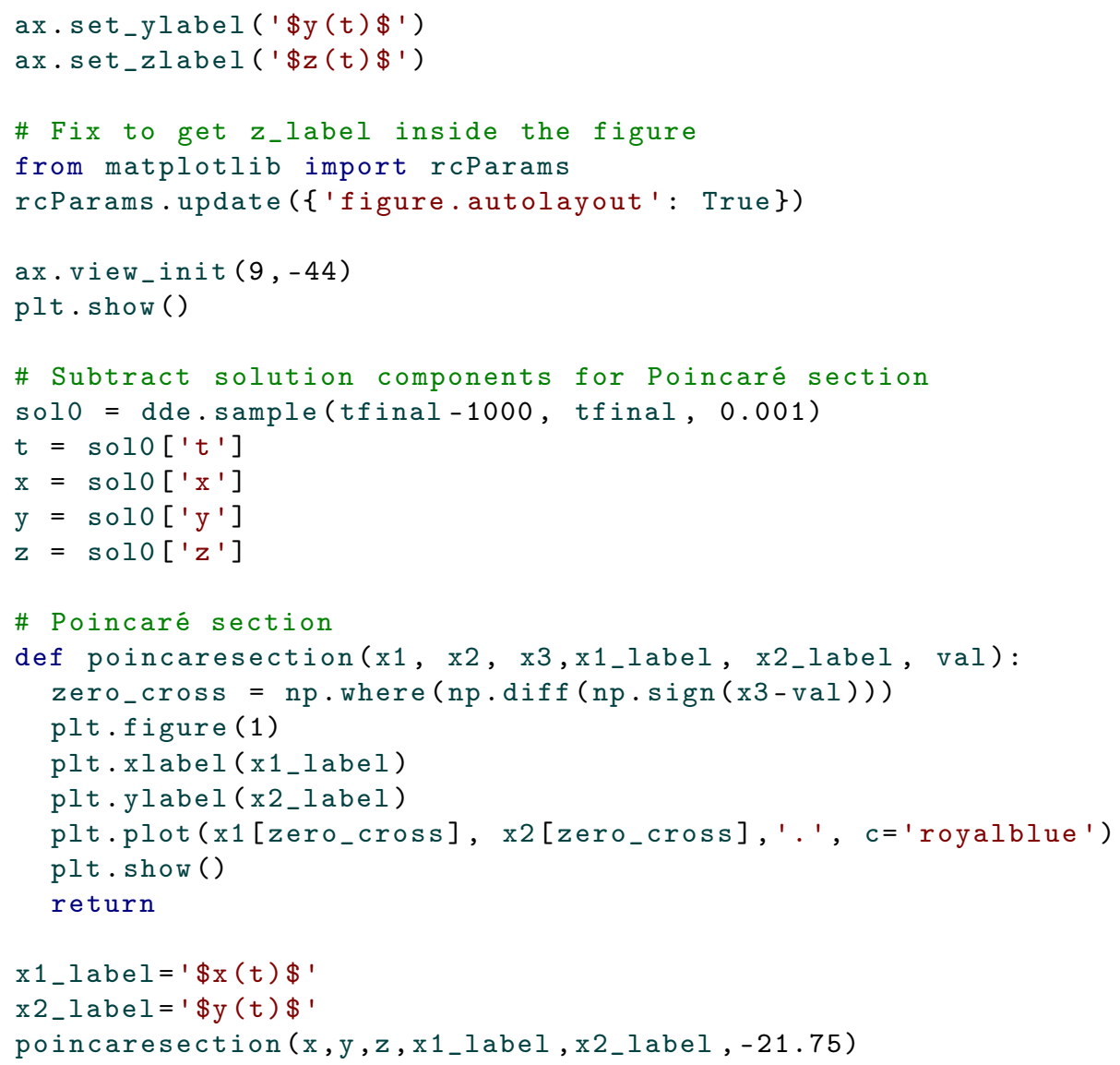

We will simulate the dynamics in region I and II, see Figure S7, where a stable periodic orbit and stable two-dimensional torus, respectively, should be present. For a point in region I, we will take the unfolding parameter values

$$
\left(I_{\text {app }}, S\right)=(-18.886177304147466,-8.044197084548104) .
$$

As an initial condition, we use the constant history function with values of the location of the fold-Hopf point. We integrate the DDE on the time interval $t \in[0,10.000]$ with pydelay. In Figure S9 the time series of the components $x, y$ and $z$ and the orbit in $(x, y, z)$-space of the last 10 time steps are shown, clearly indicating a stable orbit.

Next, we simulate the dynamics in region II. Therefore, we adjust the unfolding parameter values to

$$
\left(I_{a p p}, S\right)=(-18.902420391705071,-8.045234985422740) .
$$

Furthermore, we increase the integration interval to $t \in[0,20.000]$. Keeping the history function the same, we plot the last 1000 time steps, see Figure S9. We conclude that the dynamics near the fold-Hopf point are as predicted in [43]. 


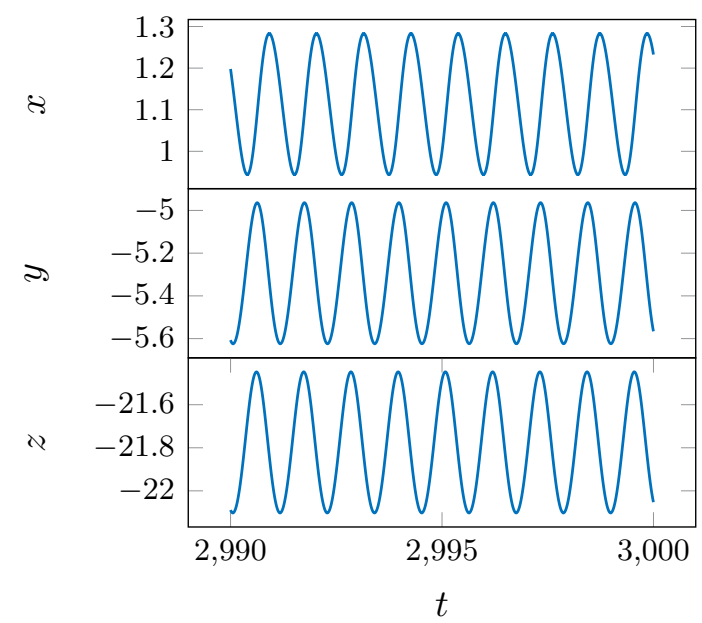

(a)

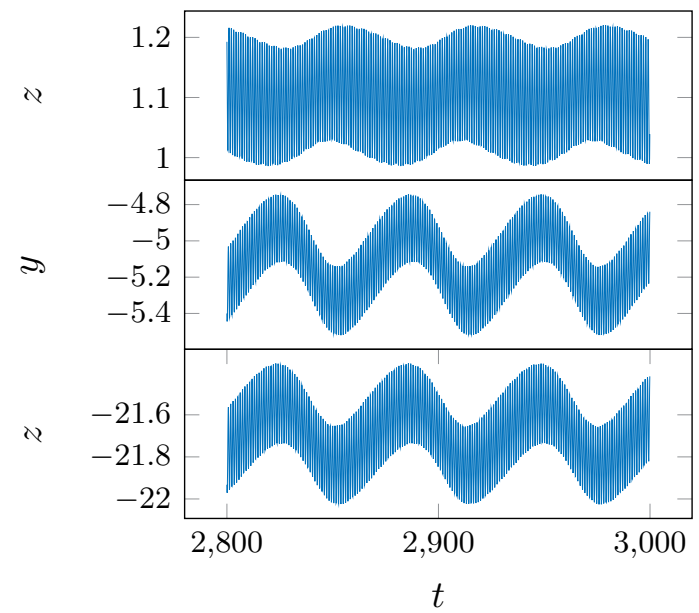

(c)

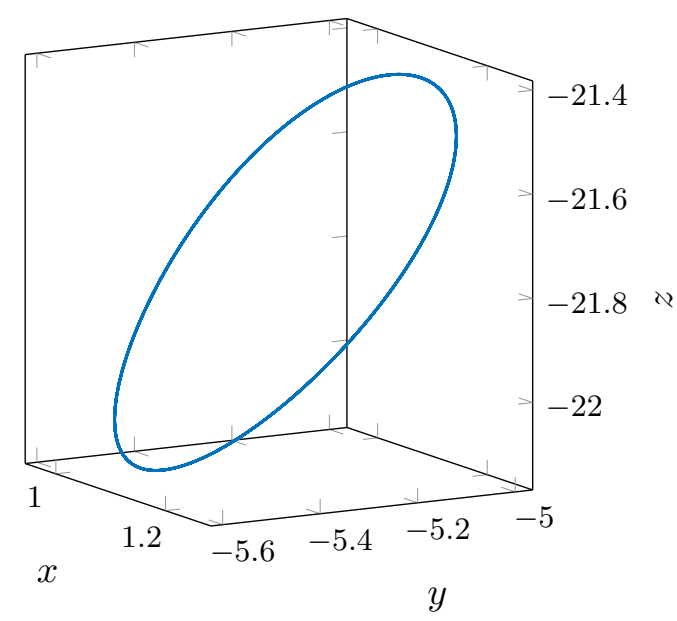

(b)

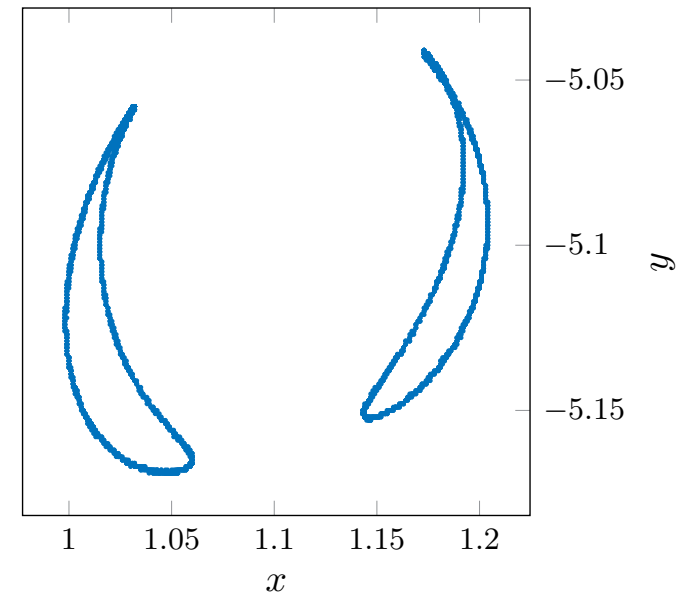

(d)

Figure S9: Simulation near fold-Hopf point in (102). In (a) and (b), a stable periodic solution is shown. In (c) and (d), a stable torus is shown. In (c), the time series is plotted while in (d), the cross-section defined by $z(t)=-21.75$ in the phase-space $(x, y, z)$ is taken.

\section{S3 Hopf-Hopf and generalized Hopf bifurcations in Active con- trol system}

In Section 8.3 we considered the following active control system

$$
\left\{\begin{array}{l}
\dot{x}(t)=\tau y(t) \\
\dot{y}(t)=\tau\left(-x(t)-g_{u} x(t-1)-2 \zeta y(t)-g_{v} y(t-1)+f(t)\right),
\end{array}\right.
$$

which is used to control the response of structures to internal or external excitation, see [50]. The function $f$ is substituted by $\beta x^{3}(t-1)$ and the parameters

$$
g_{u}=0.1, \quad g_{v}=0.52, \quad \beta=0.1,
$$


are fixed. The control parameters are $\zeta$ and $\tau$.

Remark 25. This demonstration can be found in the directory demos/tutorial/viI/acs relative to the main directory of the DDE-BifTool package. Here, we omit the code to generate a system file. The system file sym_acs_mf.m has been generated with the script gen_acs.m. Also, we assume that the DDE-BifTool package has been loaded as in Listing S1. The code in Sections S3.1 to S3.10 highlights the important parts of the file acs.m.

\section{S3.1 Set parameter names and funcs structure}

We set the parameter names and define the funcs structure.

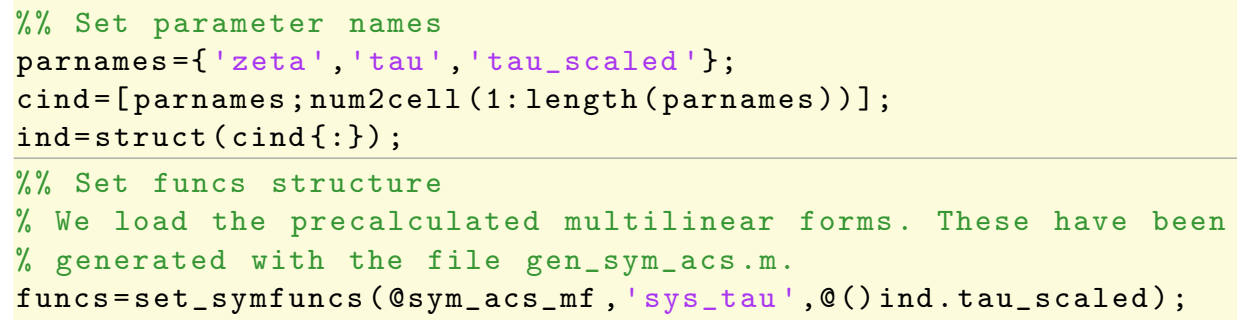

\section{S3.2 Stability and normal form coefficients of the Hopf-Hopf point}

We construct a steady-state at the Hopf-Hopf point and calculate its stability.

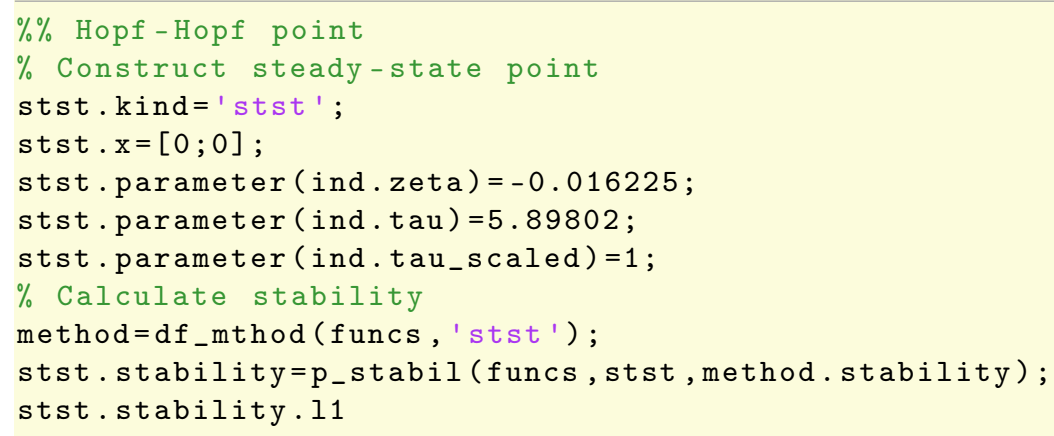

The MATLAB console shows the following output.

ans $=$

$$
\begin{array}{r}
0.0000+4.5275 i \\
0.0000-4.5275 i \\
-0.0000+7.6449 i \\
-0.0000-7.6449 i
\end{array}
$$

The eigenvalues confirm that the point under consideration is indeed a Hopf-Hopf point. Furthermore, the remaining eigenvalues have negative real parts. Next, we calculate the normal form coefficients and the transformation to the center manifold with the function nmfm_hoho, which implements the coefficients as derived in Section 6.3. For this we need to set the argument free_pars to the unfolding parameter $(\zeta, \tau)$. These coefficients will be used to start the continuation of the various branches emanating from the Hopf-Hopf point.

$\%$ Calculate coefficients of the parameter dependent normal form hopf=p_tohopf (funcs, stst);

method=df_mthod (funcs, 'hopf') ; 


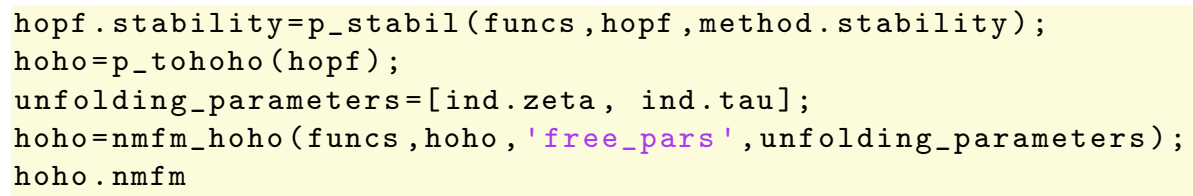

The MATLAB console shows the following output.

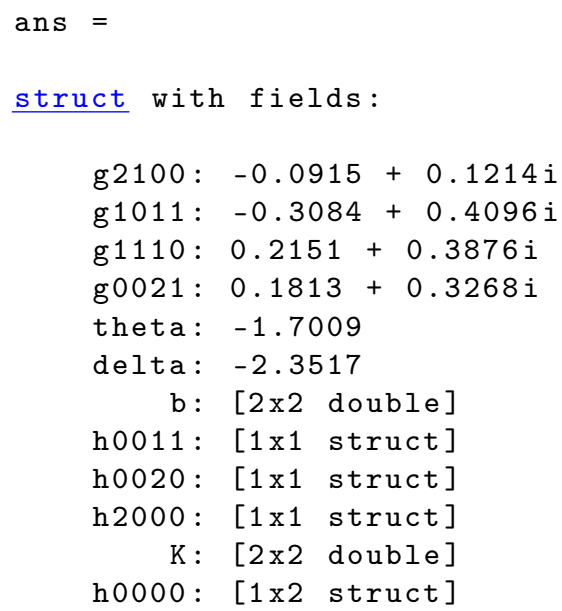

We conclude that this Hopf-Hopf bifurcation is of 'difficult' type, since

$$
\left(\operatorname{Re} g_{2100}\right)\left(\operatorname{Re} g_{0021}\right)=-0.0166<0,
$$

see [43]. Furthermore, the quantities

$$
\theta=\frac{\operatorname{Re} g_{1011}}{\operatorname{Re} g_{0021}}=-1.7009, \quad \delta=\frac{\operatorname{Re} g_{1101}}{\operatorname{Re} g_{2100}}=-2.3517
$$

are such that $\theta<0, \delta<0, \theta \delta>0$. It follows that we are in case VI.

\section{S3.3 Set bifurcation parameter range and step size bounds}

Before continuing the various branches emanating from the transcritical-Hopf point, we create the variable brpars containing parameter bounds and maximal stepsizes.

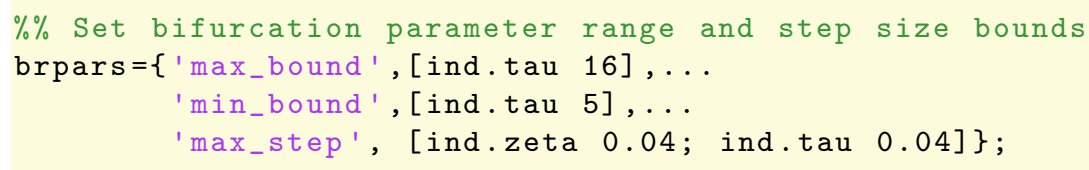

\section{S3.4 Continuing Hopf and Neimark-Sacker bifurcation curves}

We use the function C1branch_from_C2point to start to continue the branches emanating from the Hopf-Hopf point.

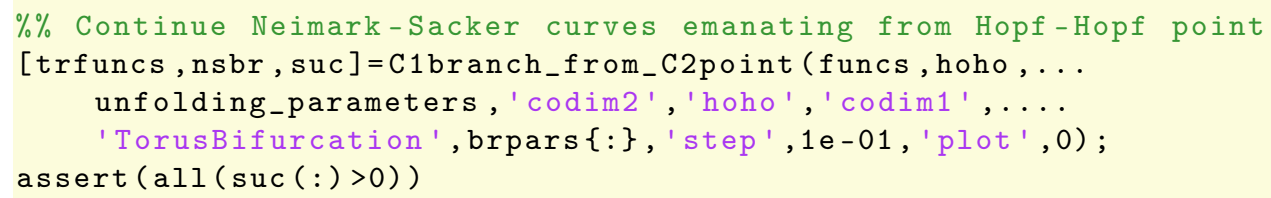




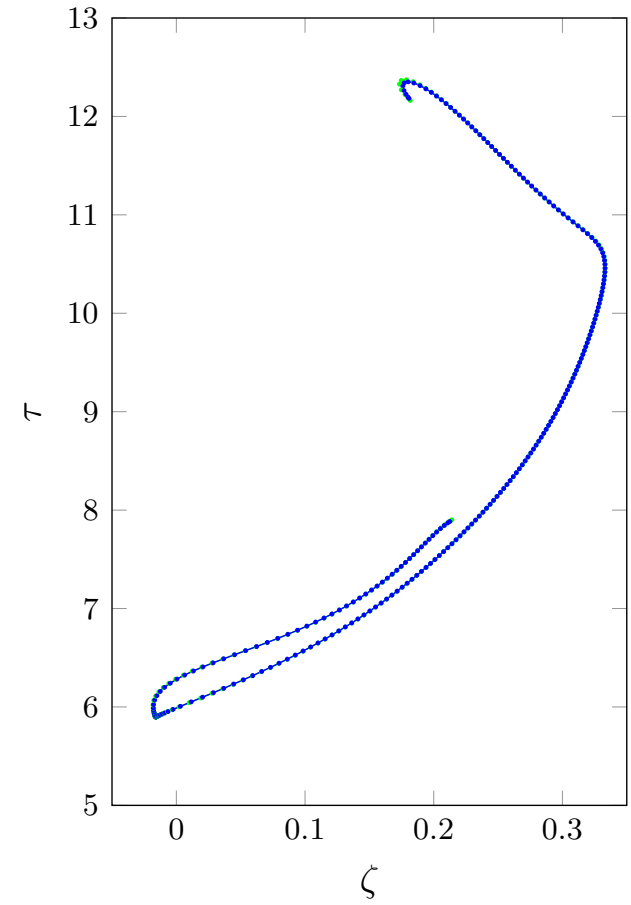

(a)

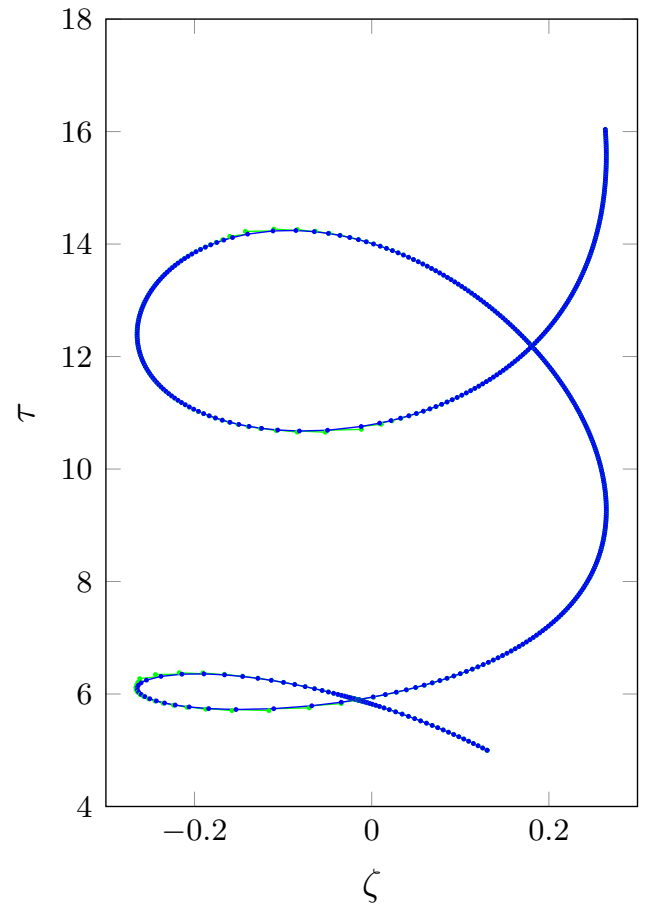

(b)

Figure S10: In (a), the continued branches ns1_br and ns2_br are plotted. In (b), the continued Hopf branch hopf_br1 is plotted.

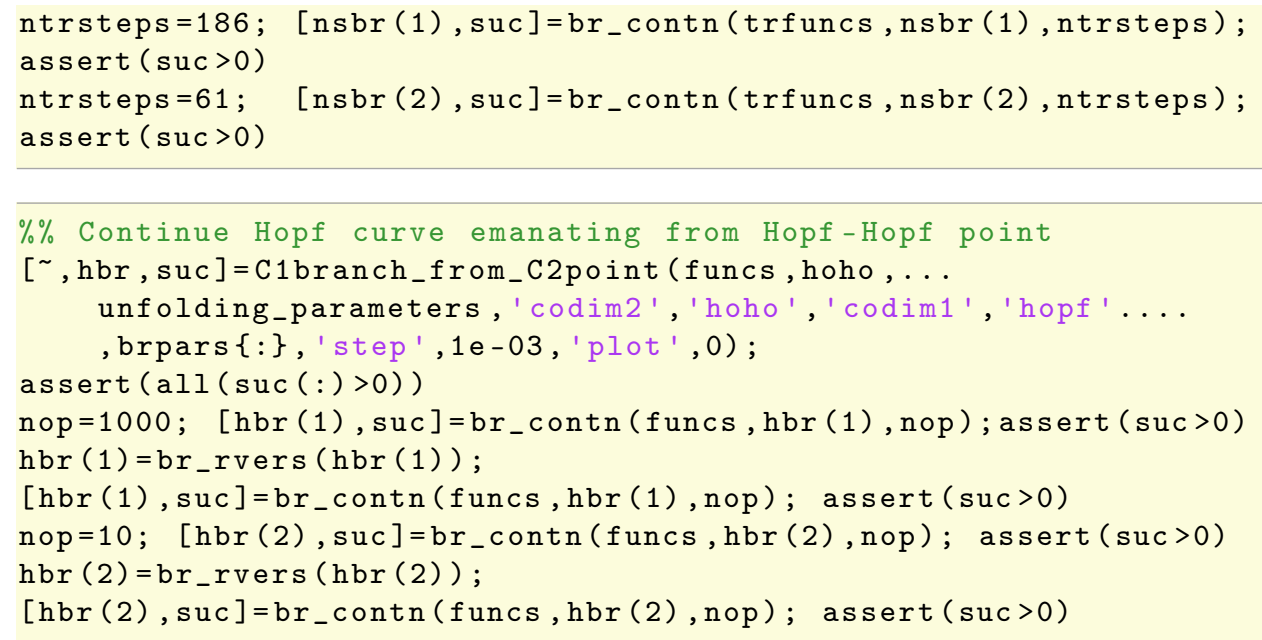

In Figure S10, the computed branches are shown. We see in Figure S10b that it is redundant to continue the second Hopf branch emanating from the Hopf-Hopf point. Indeed, the first Hopf branch connects the Hopf-Hopf point to itself. Here, we verified that the underlying points coincide. 


\section{S3.5 Predictors}

For comparison in the bifurcation diagram, we obtain predictors for the various branches by setting the argument predictor to 1 and step to a range of $\varepsilon$-values when calling the function C1branch_from_C2point.

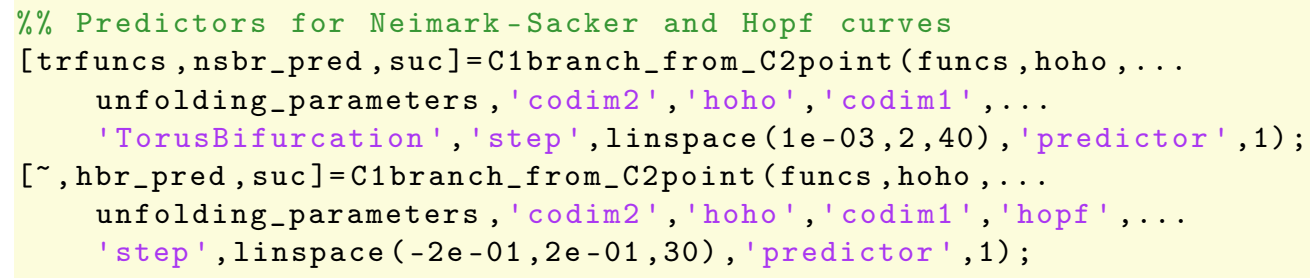

\section{S3.6 Bifurcation diagram}

We plot the computed curves and the predictors for the Neimark-Sacker and Hopf curves with the following code.

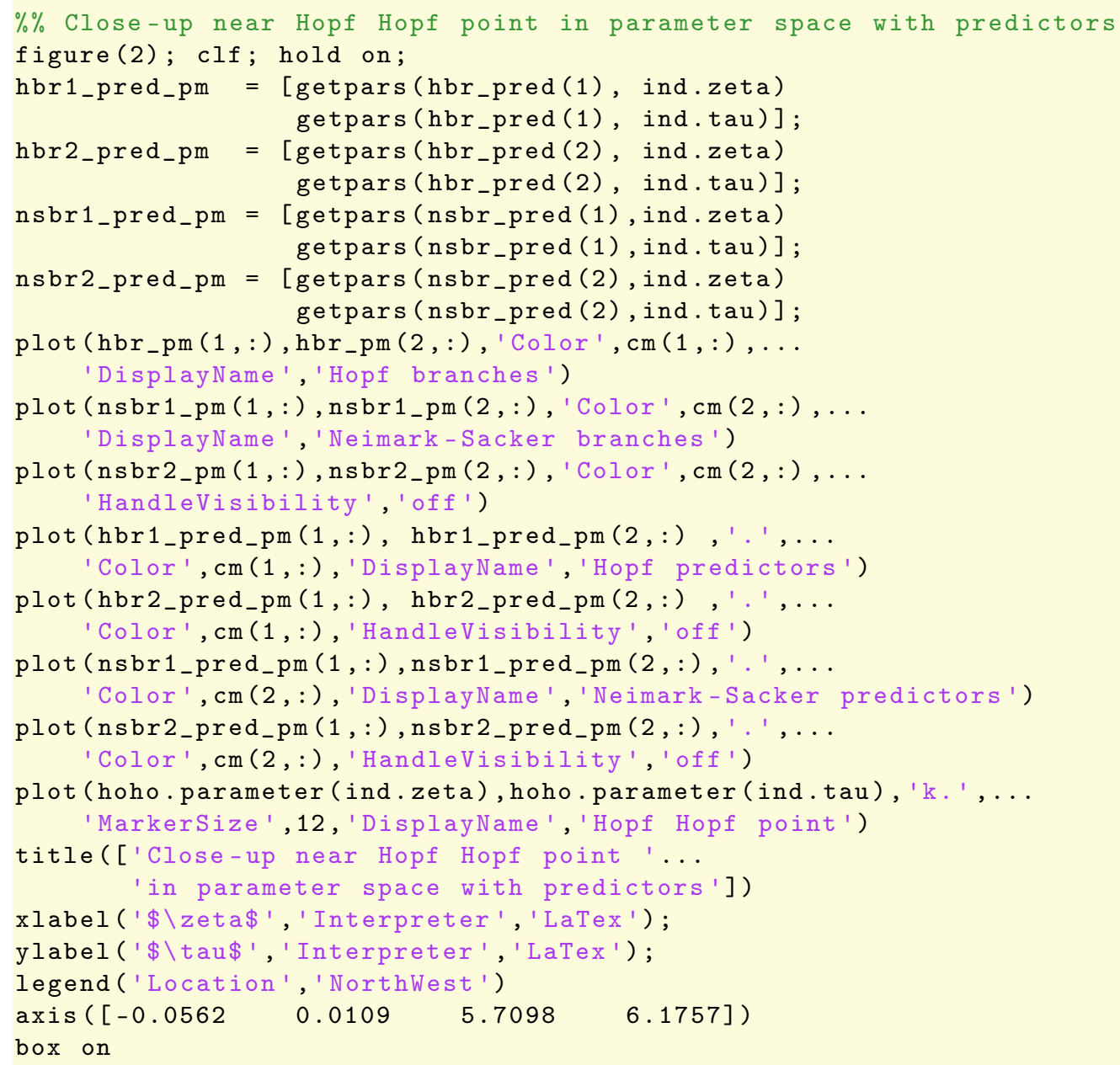

In Figure S11a the predictors in parameter space are compared. 


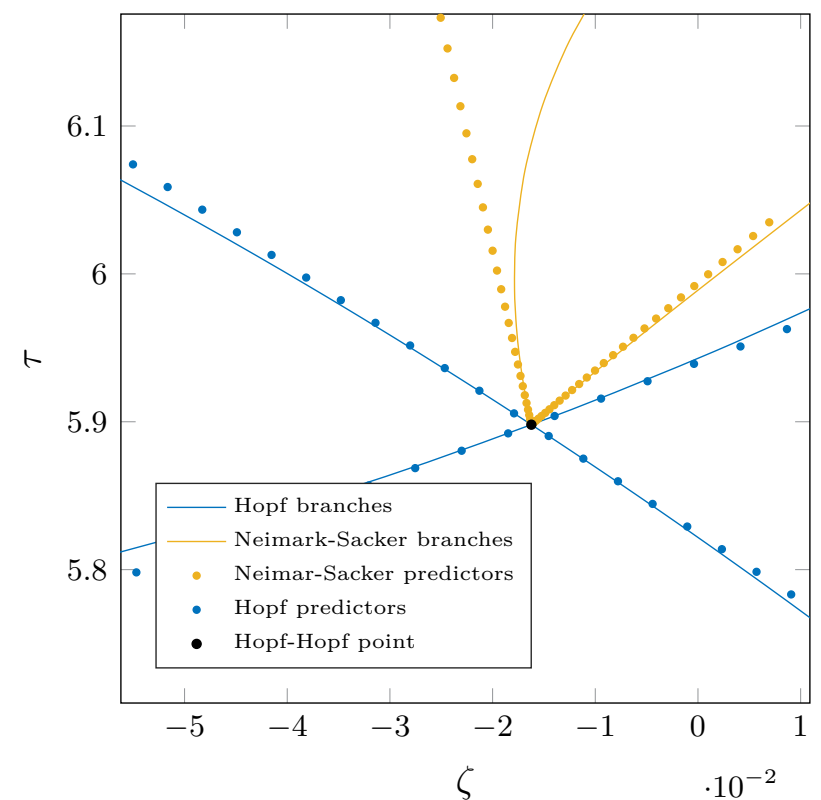

(a)

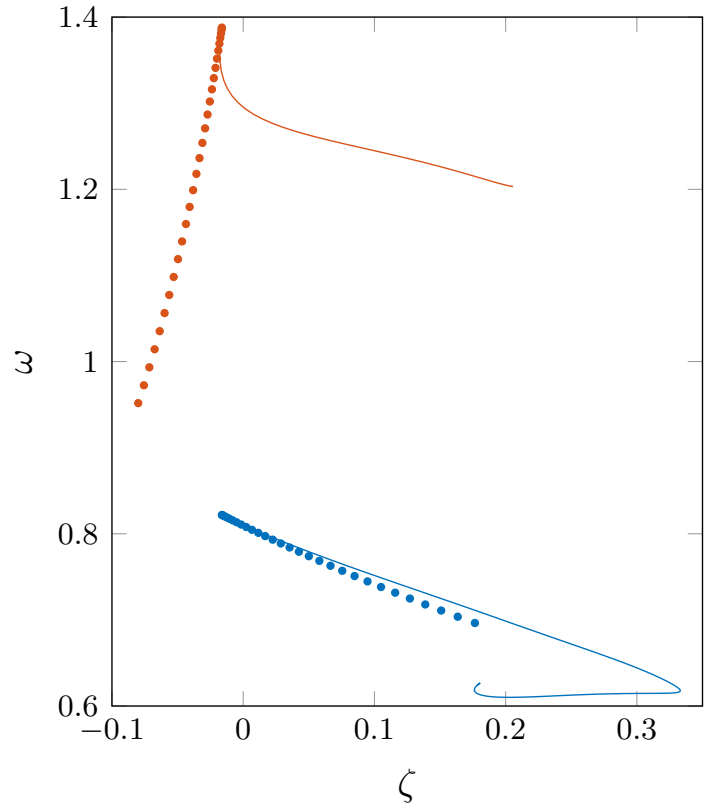

(b)

Figure S11: In (a), the computed curves are compared with the predicted curves for the Hopf-Hopf point hoho. In (b), the computed and predicted periods of the cycles are compared.

\section{S3.7 Plot comparing computed and predicted periods}

To compare the computed and predicted periods, we use the following code.

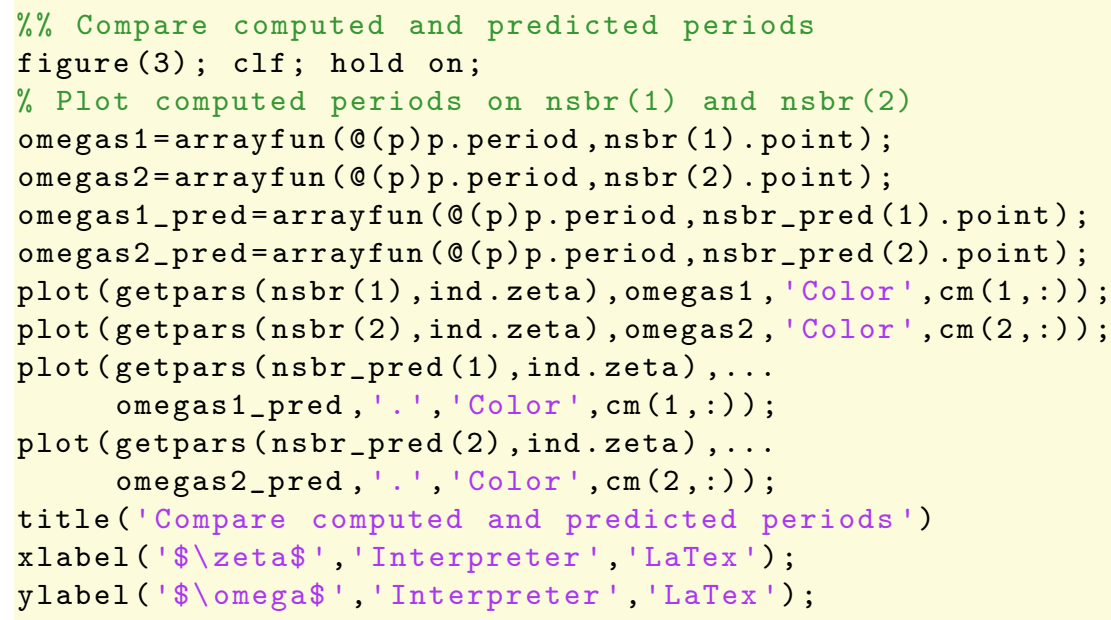

In Figure S11b, the computed and predicted periods of the cycles are compared using the formulas as given in Section 5.3.2.

\section{S3.8 Detect special points on the Hopf branch}

Using the detection capabilities from DDE-BifTool via the function LocateSpecialPoints, we detect one additional Hopf-Hopf point and three generalized Hopf points. 
$\% \%$ Detect codimension two points on hopf_br 1
[hopf_br_wbifs, hopftests, hc2_indices, hc 2 _types] $=\ldots$
LocateSpecialpoints (funcs, hbr (1)) $;$

The MATLAB console shows the following output.

There are seven bifurcations detected on the Hopf branch hbr (1): four Hopf-Hopf bifurcations and three generalized Hopf bifurcations. However, by inspecting the parameters of the detected Hopf Hopf points suggest that there are only two distinct Hopf-Hopf points, which are connected with the same Hopf 
branch. By comparing the location of the underlying points confirms the premise. We are therefore not interested in continuing the Hopf curves emanating from these points. What we are interested in is continuing the Neimark-Sacker and limit point of cycle curves emanating from the second Hopf-Hopf point and the three generalized Hopf points, respectively.

The normal form coefficients of the second Hopf-Hopf point are such that

$$
\left(\operatorname{Re} g_{2100}\right)\left(\operatorname{Re} g_{0021}\right)=1.7331 e-04>0
$$

and

$$
\theta \geq \delta>0, \quad \delta \theta=4>1 .
$$

We conclude that we are in case I of the 'simple' type, see [46, page 360]. Therefore, no stable invariant two-dimensional torus is predicted for nearby parameter values. We only expect to find two stable periodic orbits.

\section{S3.9 Continuing third Neimark-Sacker bifurcation and limit point of cycle bifurcation curves}

It turns out that we only need to continue one of the Neimark-Sacker bifurcation curves emanating from the second Hopf-Hopf point. Indeed, the other Neimark-Sacker bifurcation curve is given by ns_br(1).

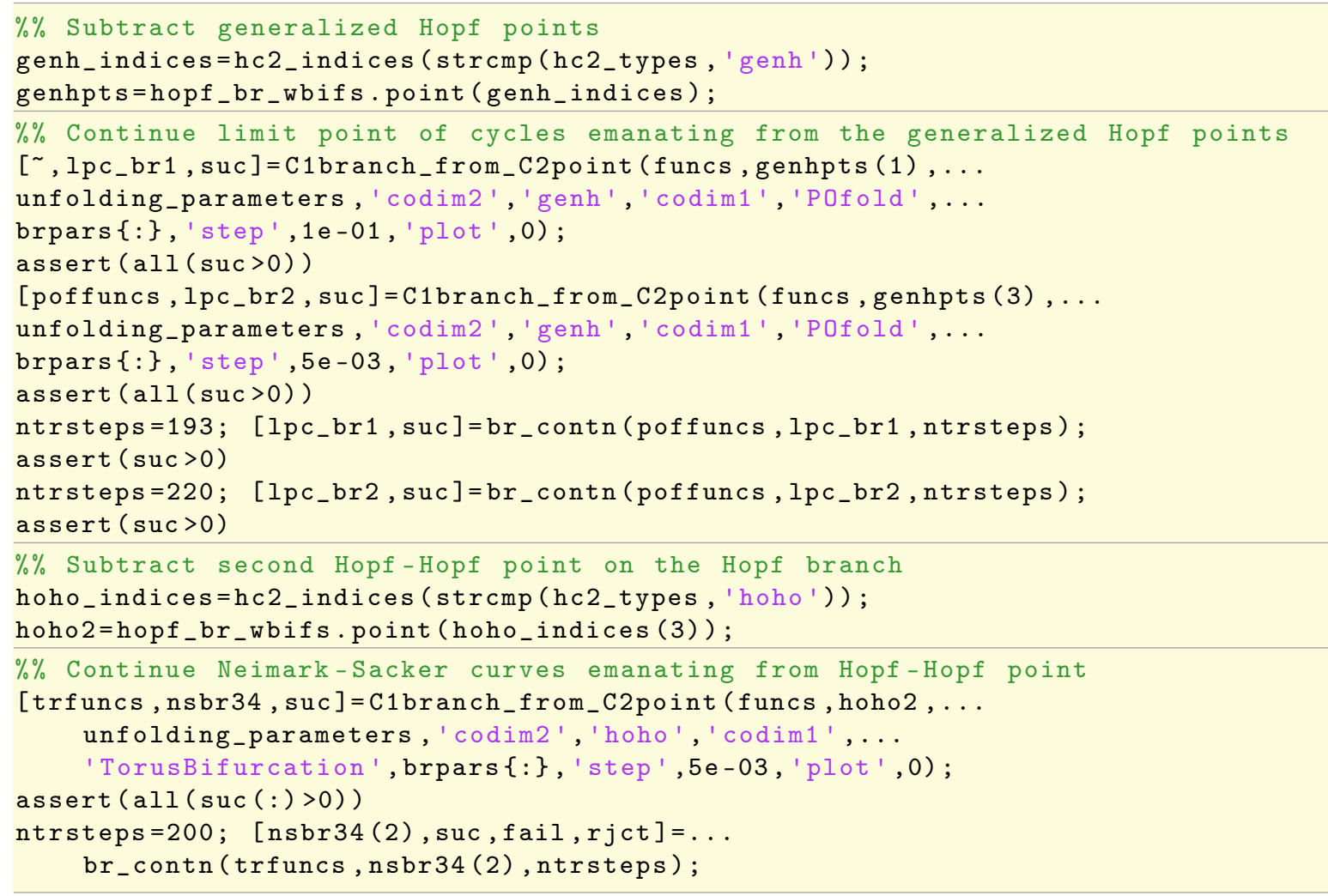

\section{S3.10 Bifurcation diagram}

We plot the computed degenerate Hopf points, the limit point of limit cycle curves, and the NeimarkSacker curves with the following code. 


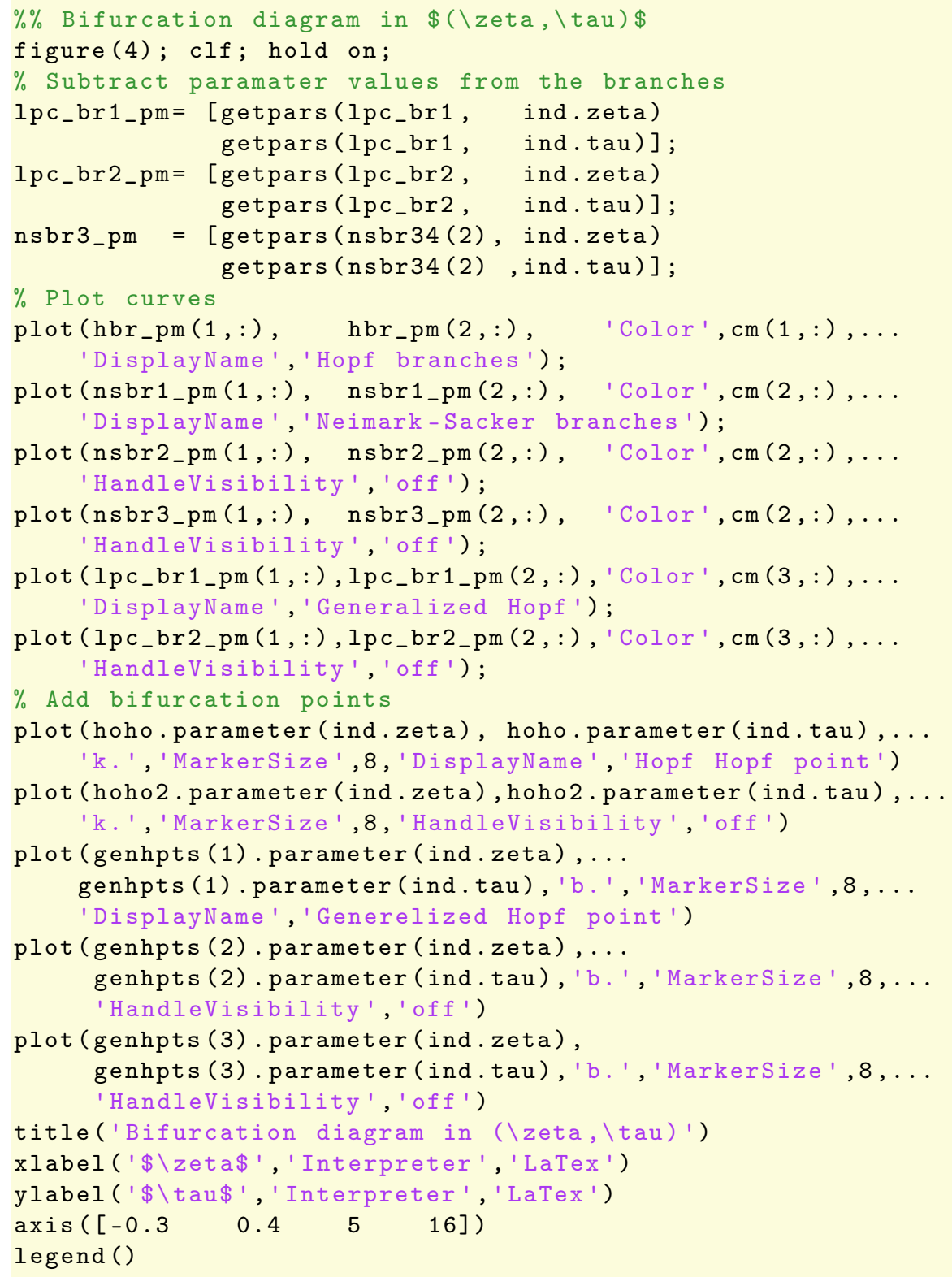

Figure S12 shows the resulting bifurcation diagram. There, we see that two generalized Hopf points are connected by the same limit point of cycles bifurcation curve.

\section{S3.11 Simulation near Hopf-Hopf point with pydelay}

In this last Section, we simulate the dynamics near the manually constructed Hopf-Hopf point at parameter values (109). As remarked before, the unfolding of the Hopf-Hopf point hoho is of 'difficult' type case VI. The normal form coefficients predict a stable invariant two-dimensional torus. Furthermore, this torus undergoes a bifurcation in which a three-dimensional torus is born. Since the DDE under investigation (108) does not contain any terms of order higher than three, the results for the normal form remain valid for the system. To confirm the unfolding in Figure 3, we fix the delay 


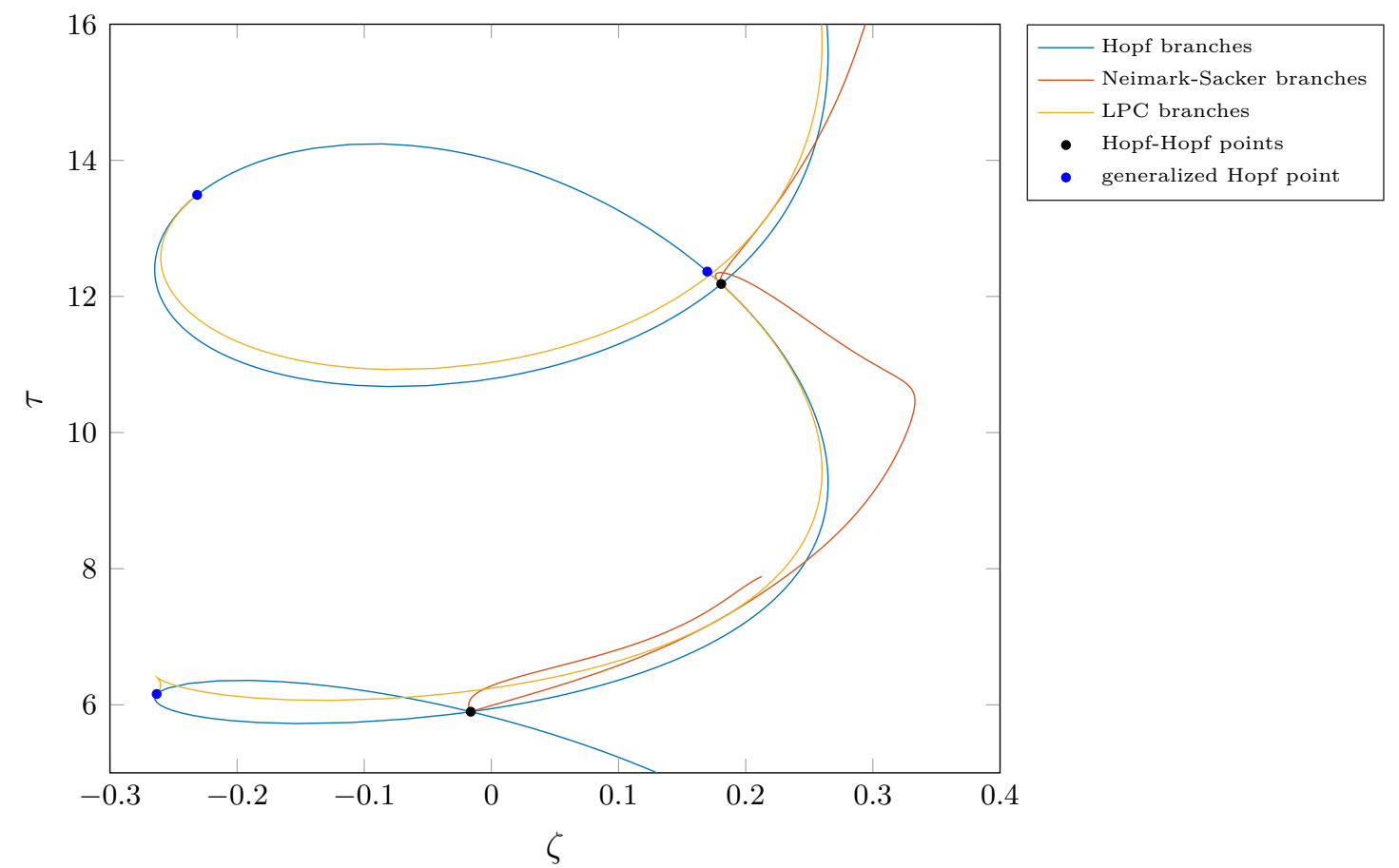

Figure S12: Unfolding from multiple codimension two points detected in the DDE (S4).

$\tau=5.901783308978358$ and take for $\zeta$ consecutive the values

$$
\begin{aligned}
& \zeta_{1}=-0.015485728828307, \\
& \zeta_{2}=\zeta_{1}-0.0002, \\
& \zeta_{3}=\zeta_{1}-0.0004, \\
& \zeta_{4}=\zeta_{1}-0.000445, \\
& \zeta_{5}=\zeta_{1}-0.0004496,
\end{aligned}
$$

see Figure S13a. The cross-sections in Figures S13b and S14d are generated with the following Python code using pydelay, see also the file acs_simulation.py

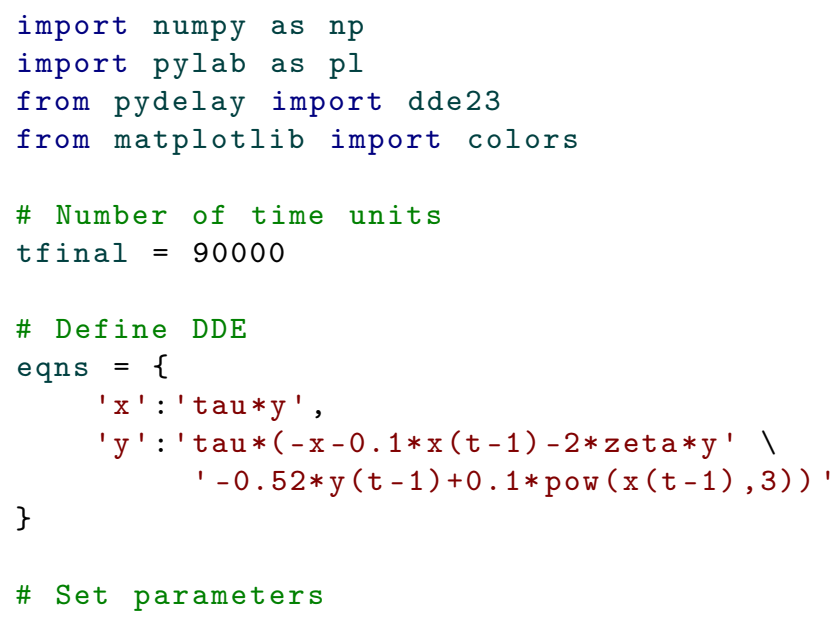




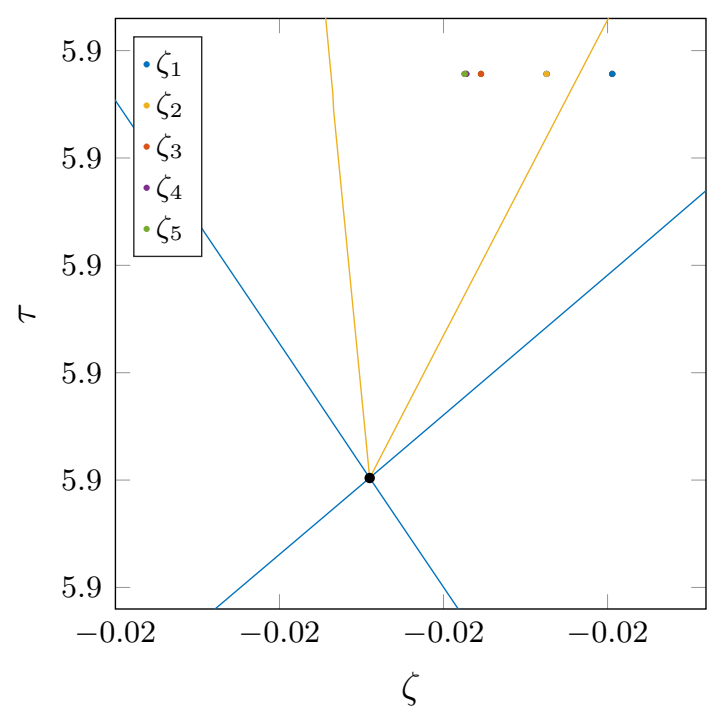

(a) Bifurcation diagram near Hopf-Hopf point

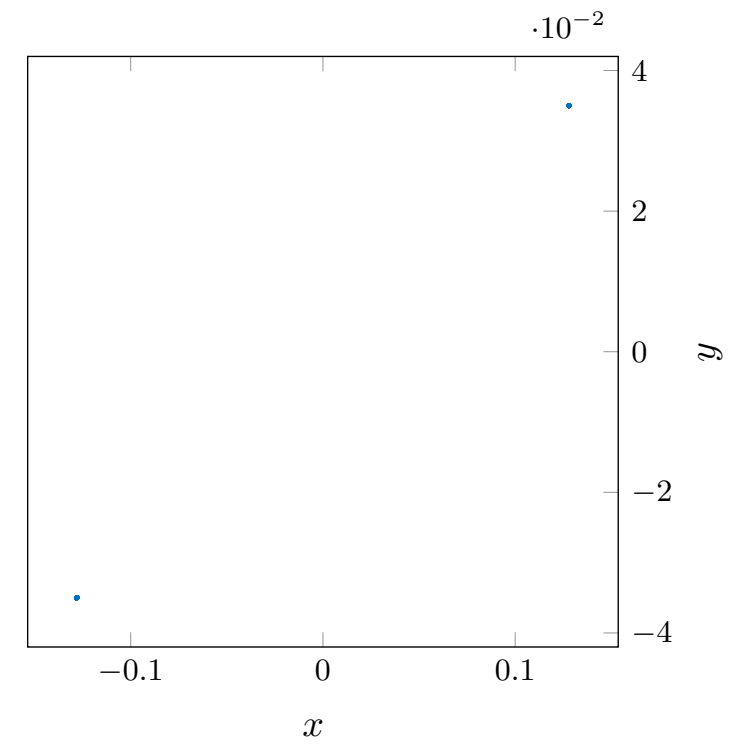

(b) $\zeta_{1}$ Stable periodic orbit

Figure S13: In (a), the points $\left(\zeta_{i}, \tau\right)$ for $i=1, \ldots, 5$ of the parameter values where the simulation is performed are plotted. Note that $\zeta_{4}$ and $\zeta_{5}$ are almost indistinguishable. In (b), the Poincaré section of the simulation using pydelay at the parameters $\left(\zeta_{2}, \tau\right)=(-0.015685728828307,5.901783308978358)$ shows a stable periodic orbit.

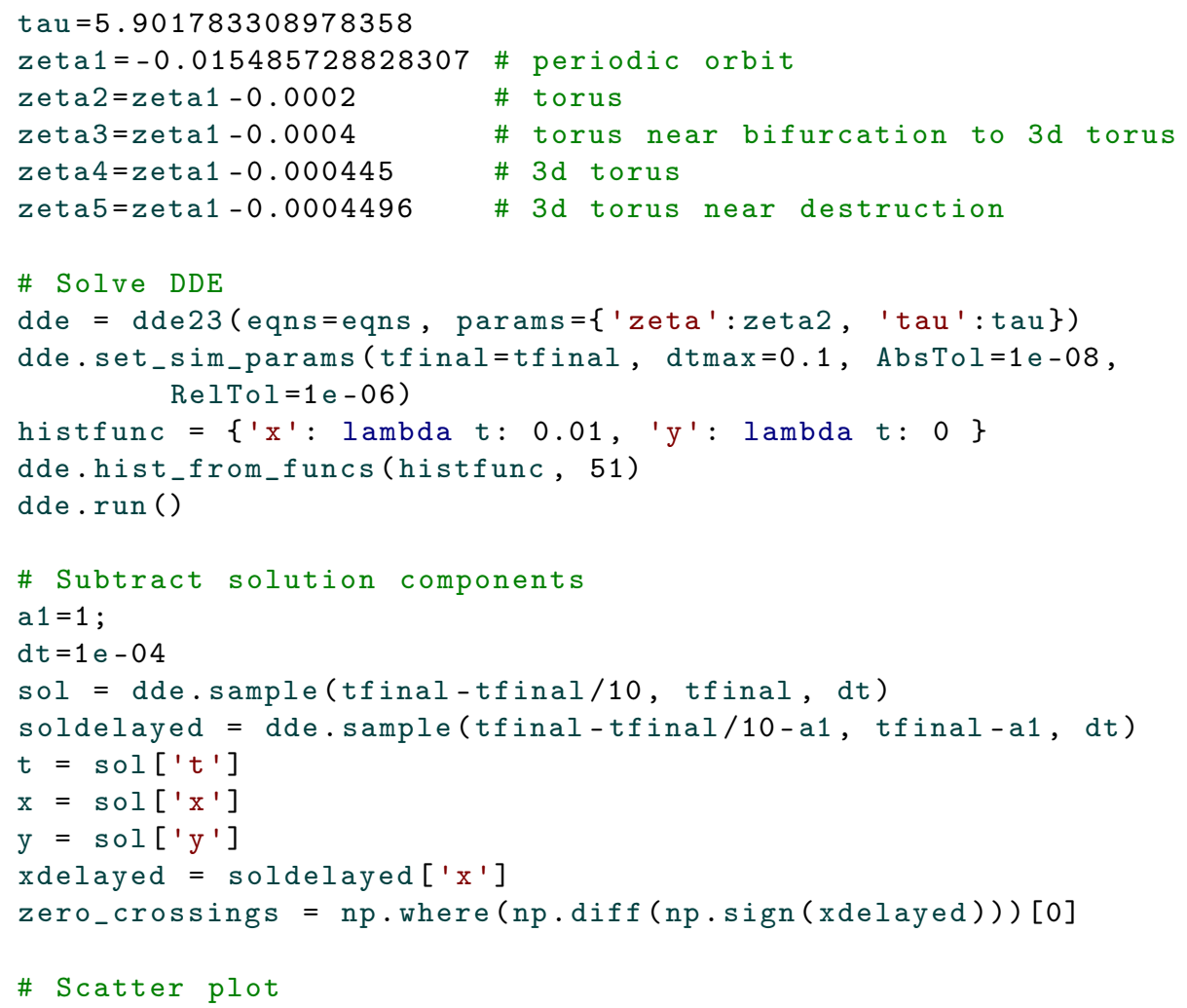




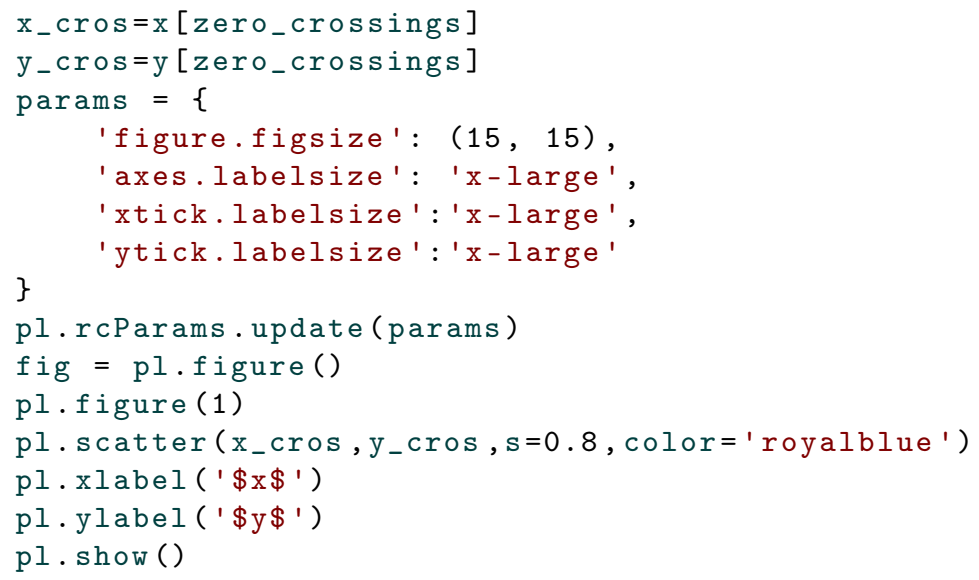

In Figure S13b there are two dots, corresponding to a stable period orbit. Crossing the curve ns1_br, a stable two-dimensional torus branches off, see Figure S14a. The torus still exists at $\zeta=\zeta_{3}$, as seen in Figure S14b. Then, at $\zeta=\zeta_{4}$, only slightly smaller than $\zeta_{3}$, a three-dimensional torus is observed, see Figure S14c. Lastly, Figure S14d shows the three-dimensional torus near the curve where the torus blows up.

\section{S4 Transcritical-Hopf bifurcation in Van der Pol's oscillator with delayed position and velocity feedback}

Consider the generalized van der Pol's oscillator with delayed feedback

$$
\left\{\begin{aligned}
\dot{x}(t)= & \left(\tau_{0}+\mu_{2}\right) y(t), \\
\dot{y}(t)= & \left(\tau_{0}+\mu_{2}\right)\left[-x(t)-\varepsilon\left(x^{2}(t)-1\right) y(t)+\left(1+\mu_{1}\right) x(t-\tau)-0.2 y(t-1)\right. \\
& \left.\quad-0.2 x^{2}(t-1)-0.2 x(t-\tau) y(t-1)-0.2 y^{2}(t-1)+0.5 x^{3}(t-1)\right],
\end{aligned}\right.
$$

see Section 8.4 and [3]. The parameter $\varepsilon=0.3$ is fixed. For $\tau_{0} \approx 1.757290761249588$ a transcritical-Hopf bifurcation is located at $\left(\mu_{1}, \mu_{2}\right)=(0,0)$.

Remark 26. This demonstration can be found in the directory demos/tutorial/VII/vdpo relative to the main directory of the DDE-BifTool package. Here, we omit the code to generate a system file. The system file sym_vdpo_mf.m has been generated with the script gen_sym_vdpo.m. Also, we assume that the DDE-BifTool package has been loaded as in Listing S1. The code in Sections S4.1 to S4.8 highlights the important parts of the file vdpo.m.

\section{S4.1 Set parameter names and funcs structure}

We set the parameter names and define the funcs structure.

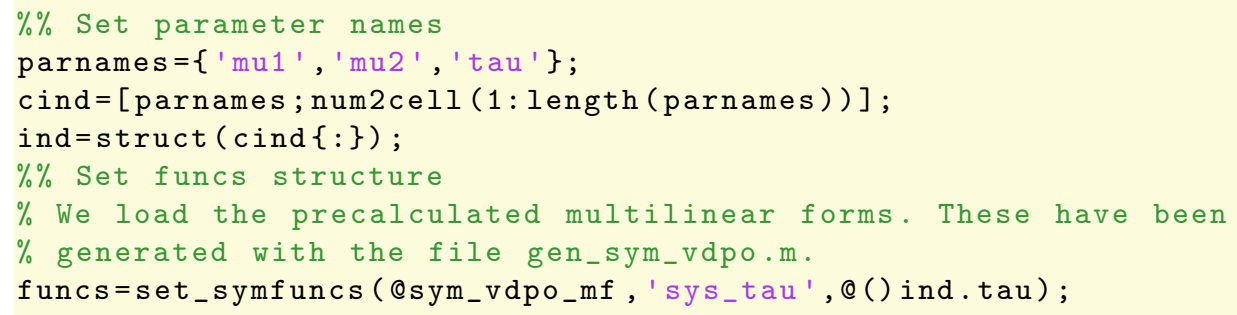




\section{S4.2 Stability and normal form coefficients of the transcritical-Hopf point}

We construct a steady-state at the transcritical-Hopf point and calculate its stability.

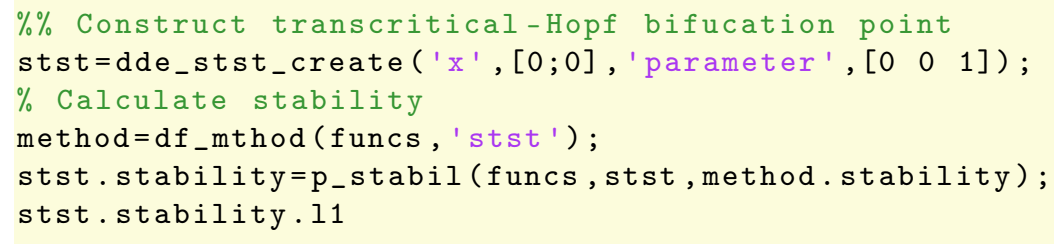

The MATLAB console shows the following output.

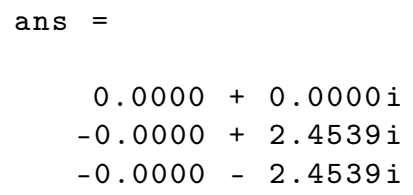

We have a zero eigenvalue and a pair of purely imaginary eigenvalues. Furthermore, the remaining eigenvalues have negative real parts. Next, we calculate the normal form coefficients and the transformation to the center manifold with the function nmfm_zeho, which implements the coefficients as derived in Sections 6.2 and 6.4. For this we need to set the argument free_pars to the unfolding parameter $\left(\mu_{1}, \mu_{2}\right)$. These coefficients will be used to start the continuation of the various branches emanating from the transcritical-Hopf point.

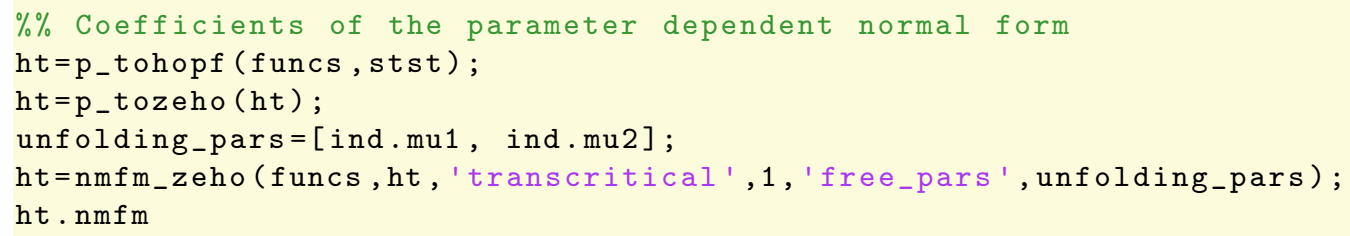

The MATLAB console shows the following output.

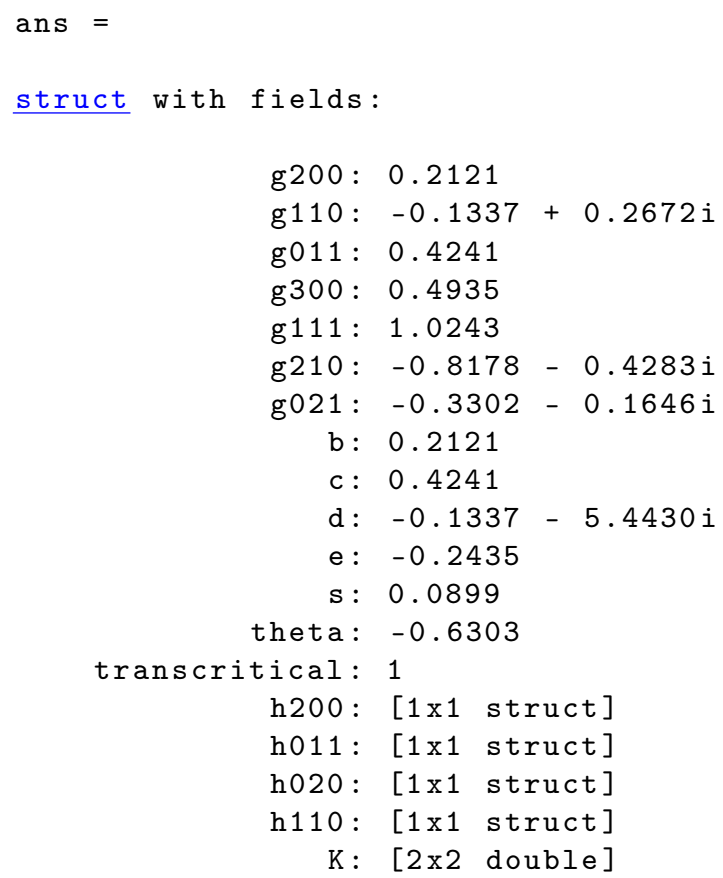


omega1: 0.4644

omega 2 : 1.2768

The normal form coefficients are such that

$$
g_{011} \operatorname{Re}\left(g_{110}\right)=0.4241 \operatorname{Re}(-0.1337+0.2672 i)<0 .
$$

Therefore, there are two Neimark-Sacker bifurcation curves predicted, see Section 5.4.

\section{S4.3 Set bifurcation parameter range and step size bounds}

Before continuing the various branches emanating from the transcritical-Hopf point, we create the variable brpars containing parameter bounds and maximal stepsizes.

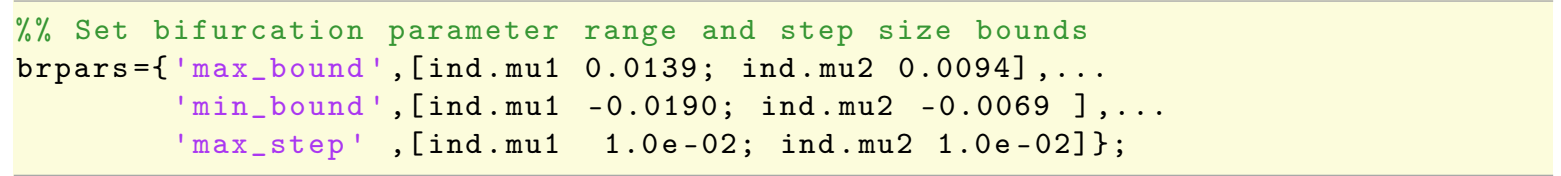

\section{S4.4 Continuing Hopf, transcritical and Neimark-Sacker bifurcation curves}

We use the function C1branch_from_C2point to continue the various branches emanating from the singularity.

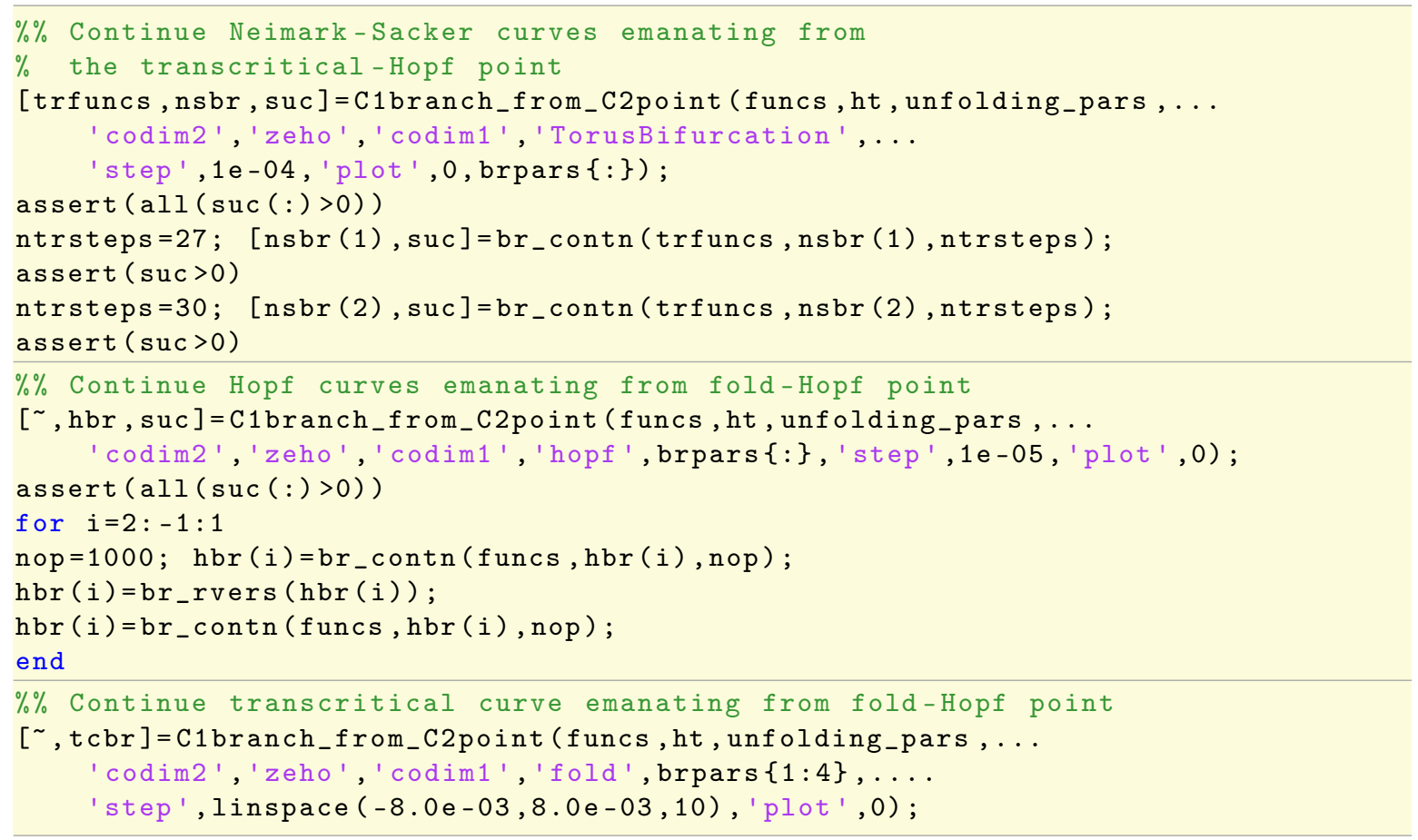

\section{S4.5 Detect special points on the Hopf branches}

We continue with detecting bifurcations on the Hopf branches. The Hopf points on the branch hbr_wbifs(i) $(i=1,2)$ will contain the normal form coefficients L1 and L2. These will be used to visualize the criticality of the Hopf points (sub or super) in the bifurcation diagram. 


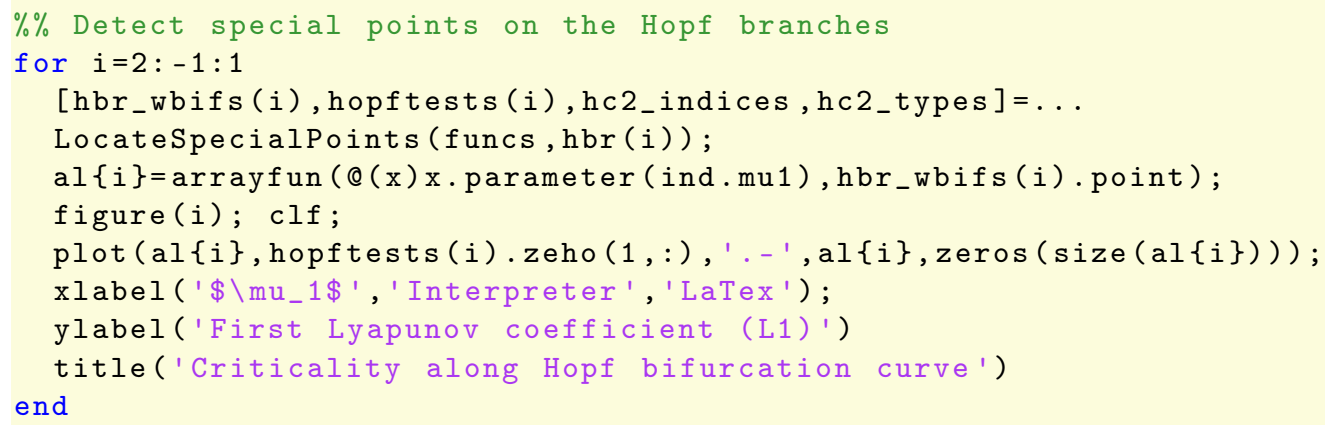

\section{S4.6 Predictors}

For comparison in the bifurcation diagram we obtain predictors for the various branches by setting the argument predictor to 1 and step to a range of $\varepsilon$-values when calling the function C1branch_from_C2point.

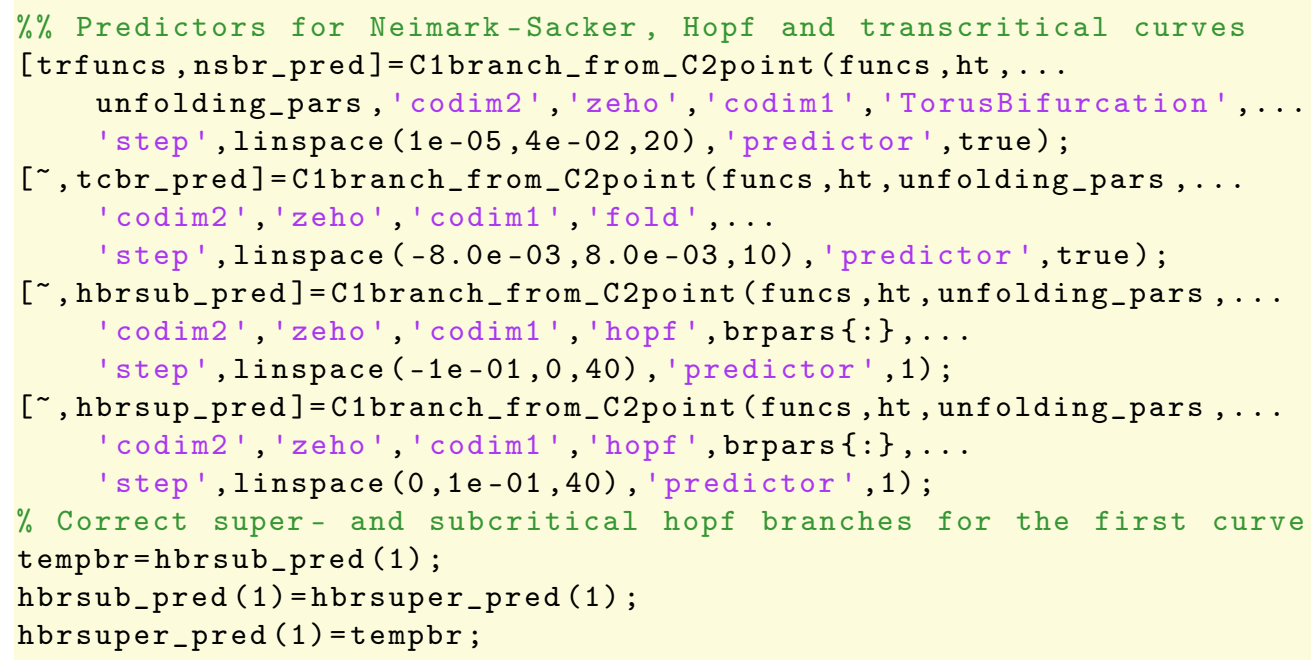

\section{S4.7 Bifurcation diagram}

We plot the obtained curves and the predictors for the Neimark-Sacker, Hopf, and transcritical curves with the following code.

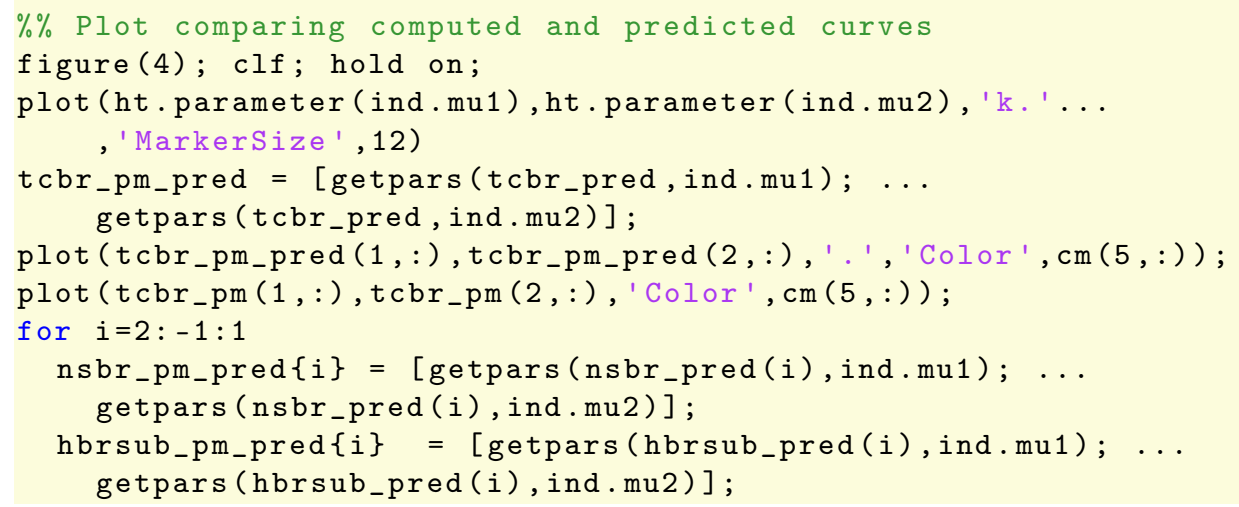




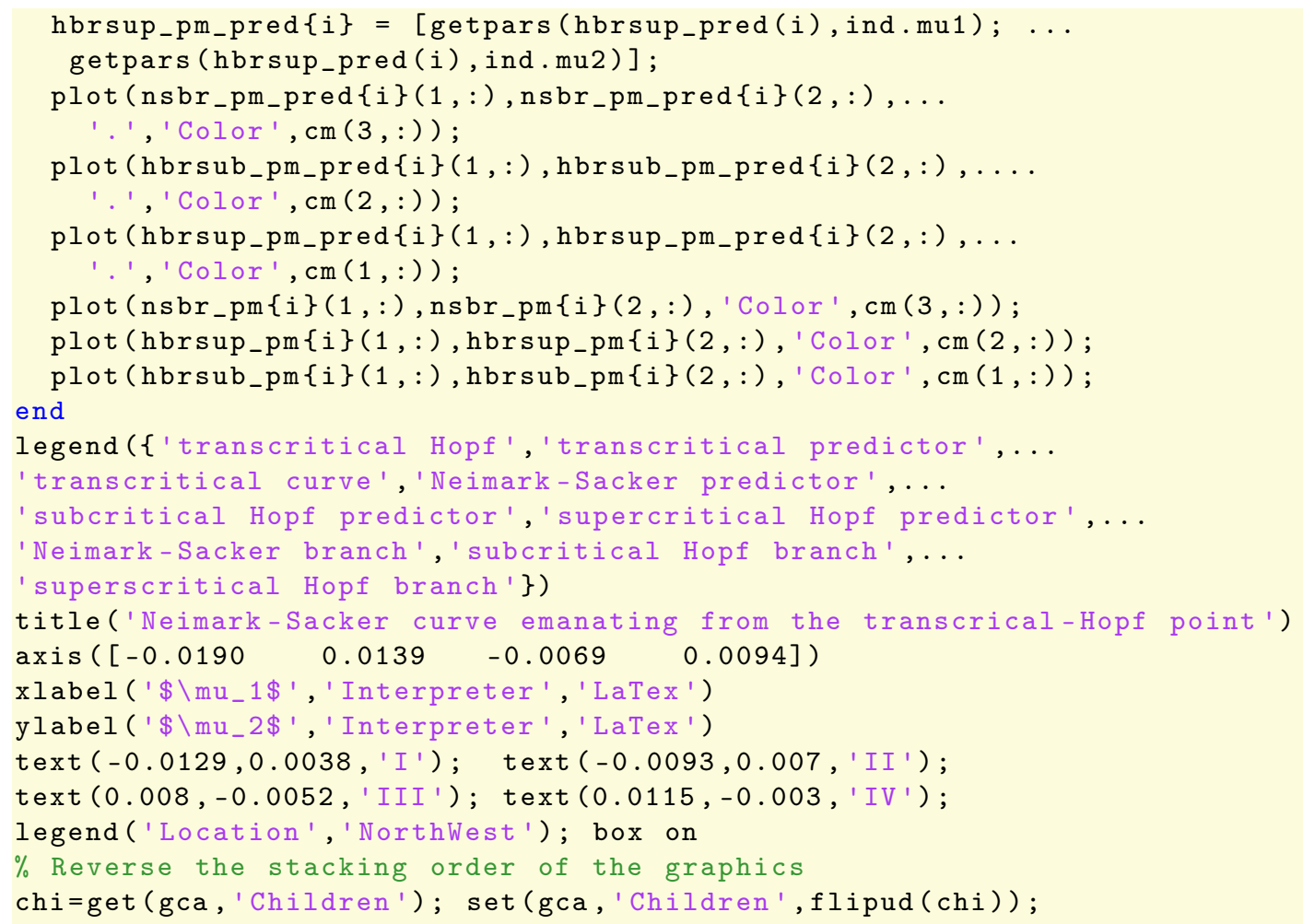

Figure S15 shows the resulting bifurcation diagram.

\section{S4.8 Plot comparing computed and predicted periodic orbits}

Lastly, we create a plot to compare the computed and predicted periodic orbits.

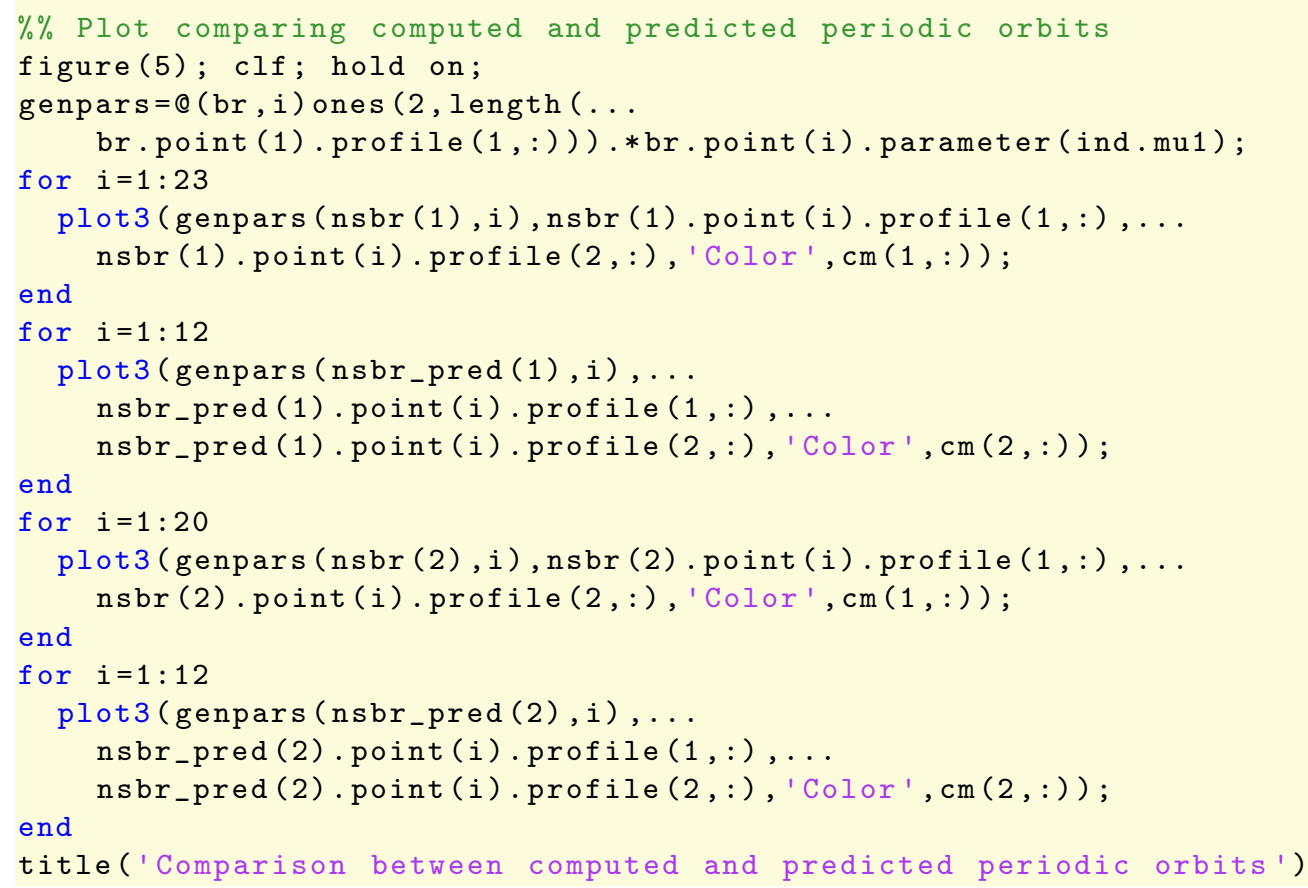


xlabel ('\mu_1'); ylabel('x'); zlabel('y');

view (3)

The resulting plot is show in Figure S16.

\section{S4.9 Simulation near transcritical-Hopf point with pydelay}

We simulate the dynamics in regions III and IV of Figure S15. Since the critical normal form coefficients are such that

$$
s=1, \quad \theta<0, \quad e<0,
$$

a stable cycle and stable torus should be present. The simulation in regions I and II have also been carried out, but have been omitted here. The following code can be found in the file vdpo_simulation.py.

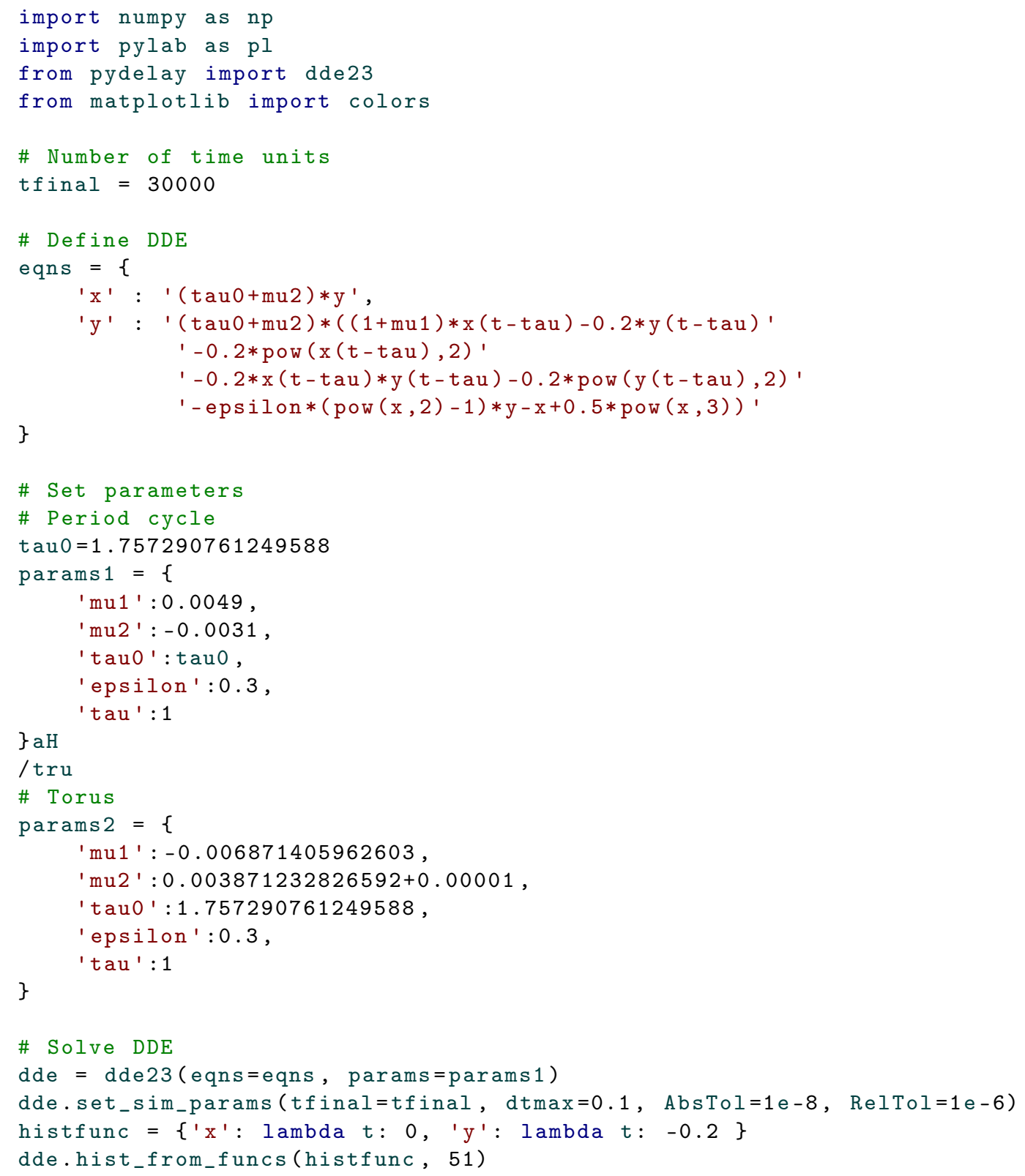




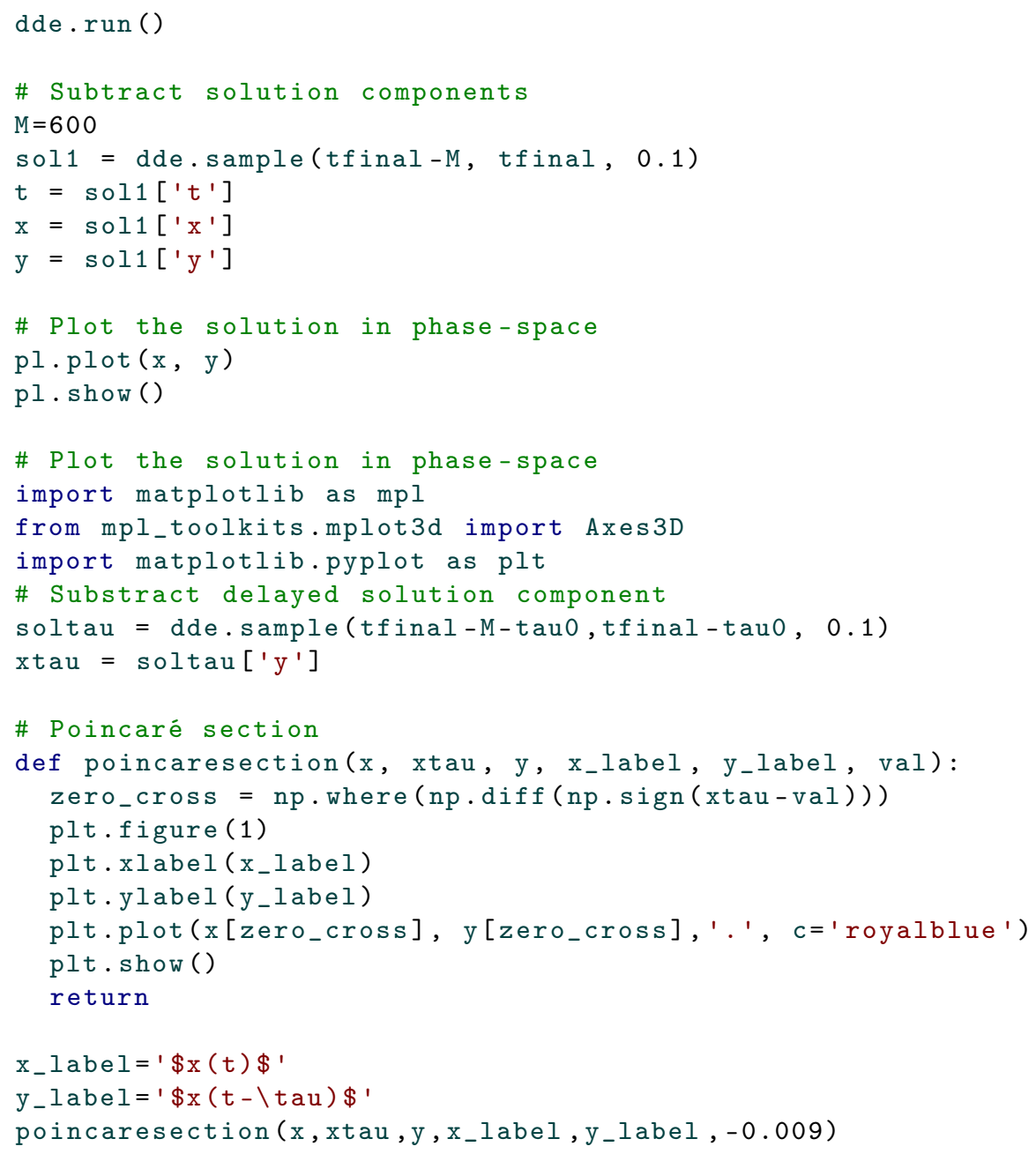




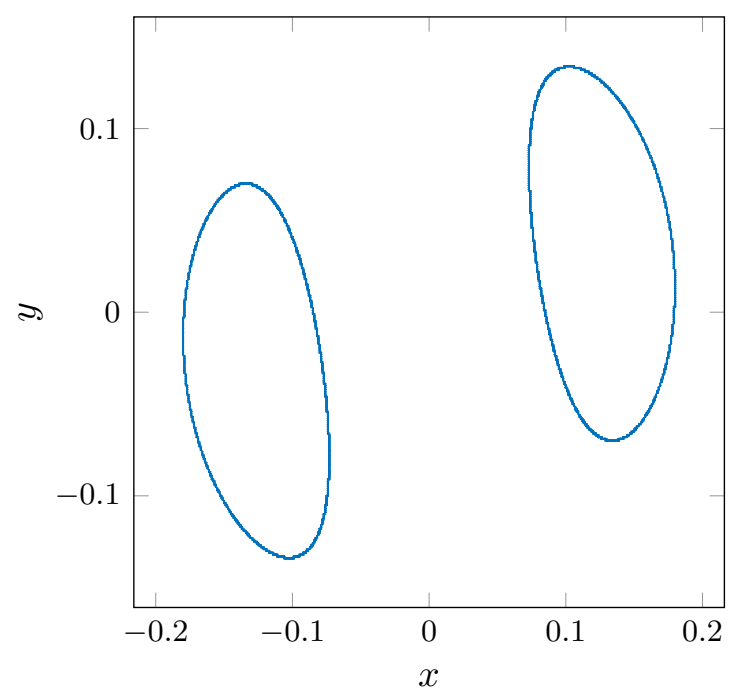

(a) $\zeta_{2}$ Stable two-dimensional torus

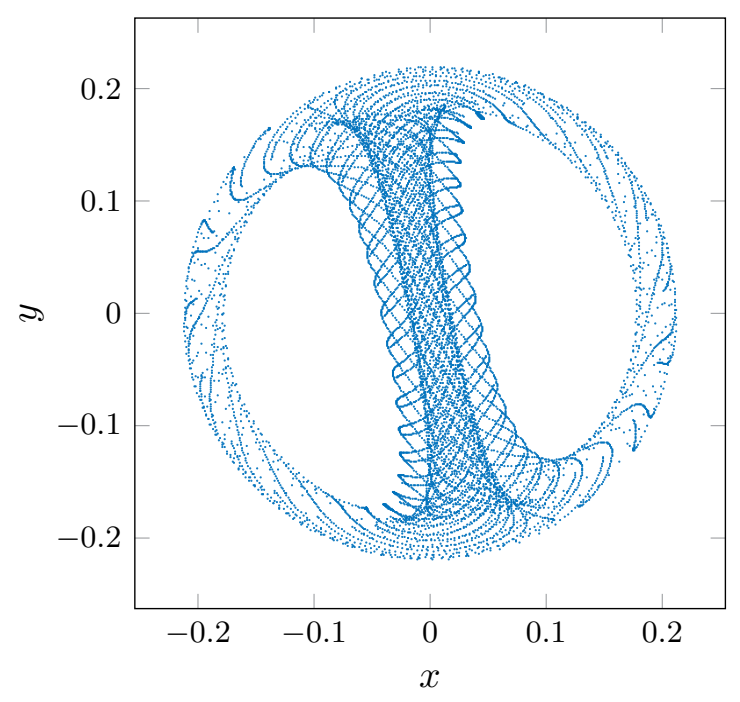

(c) $\zeta_{4}$ Stable three-dimensional torus

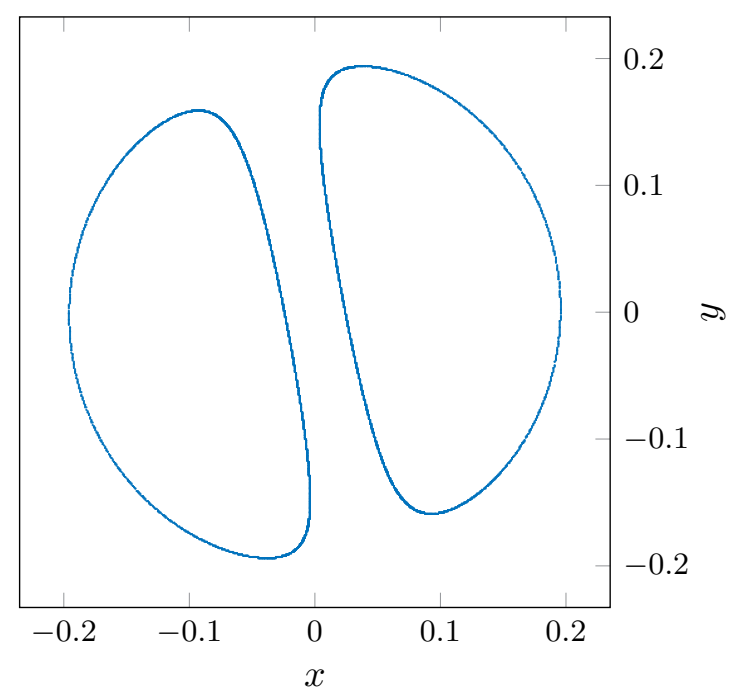

(b) $\zeta_{3}$ Stable two-dimensional torus

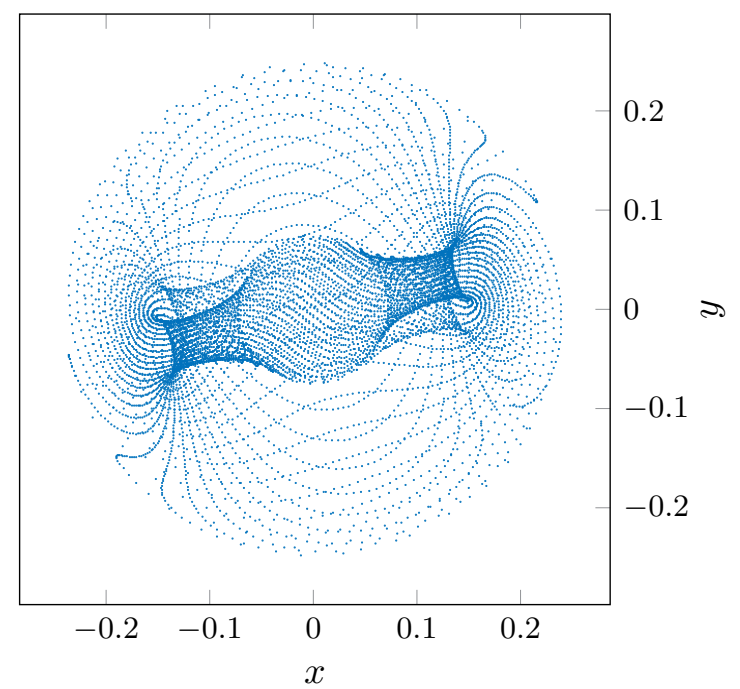

(d) $\zeta_{5}$ Three-dimensional torus near blow-up

Figure S14: Simulation with pydelay illustrating the branching of a three-dimensional torus from a two-dimensional torus. We refer to the text for further description. 


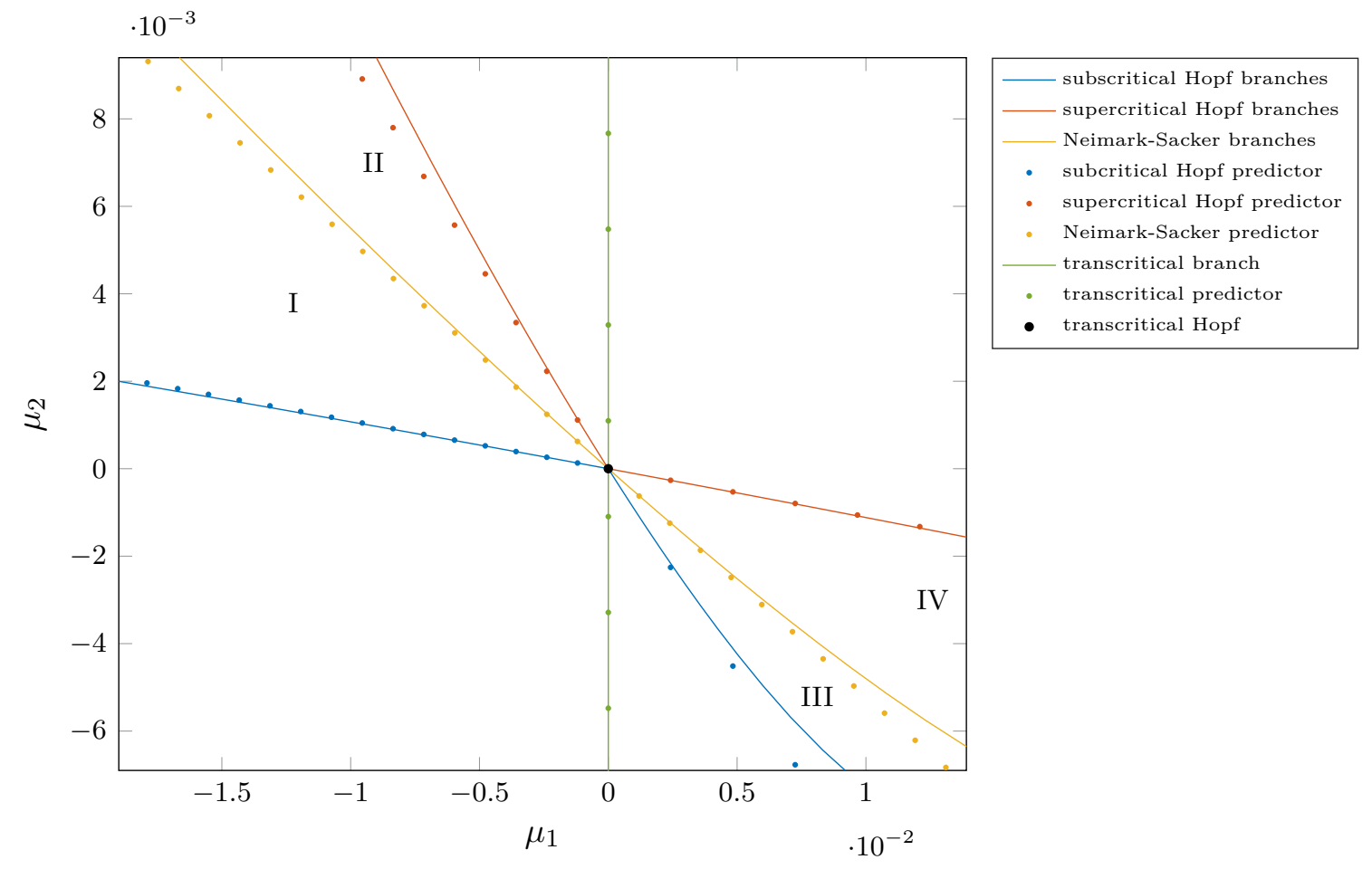

Figure S15: Bifurcation diagram near the transcritical-Hopf bifurcation in the delayed Van der Pol's oscillator given by (113). There are two supercritical Hopf curves (blue), two subcritical Hopf curves (red), two Neimark-Sacker curves (yellow), and one transcritical curve (green). We see that the predictors (dotted) give a good approximation for nearby values.

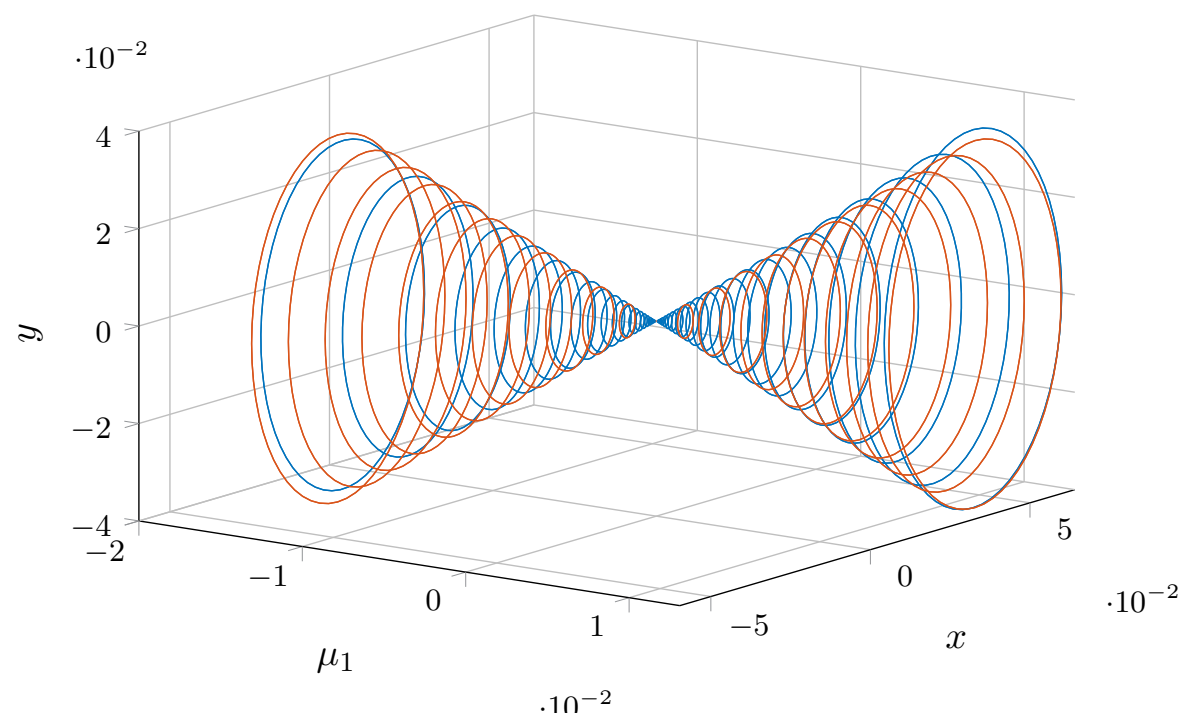

Figure S16: Comparison between predicted periodic orbits (red) and computed periodic orbits (blue) emanating from the transcritical-Hopf bifurcation 


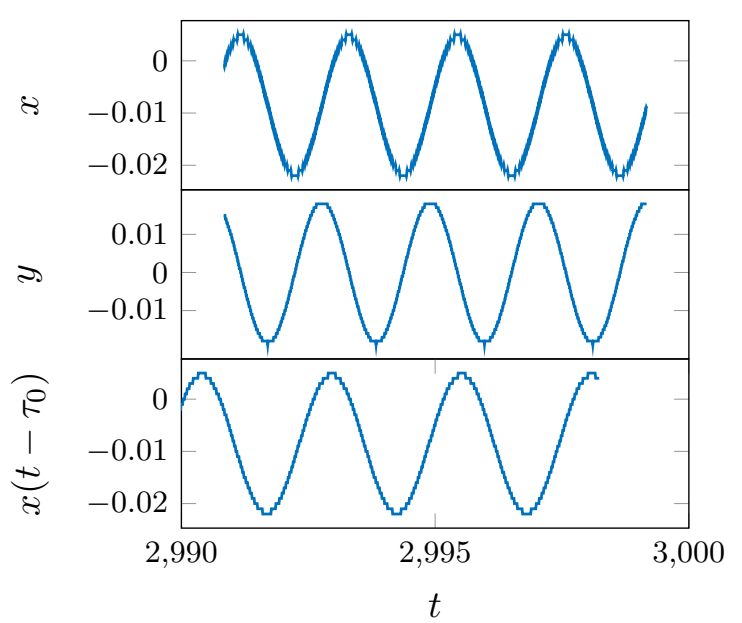

(a)

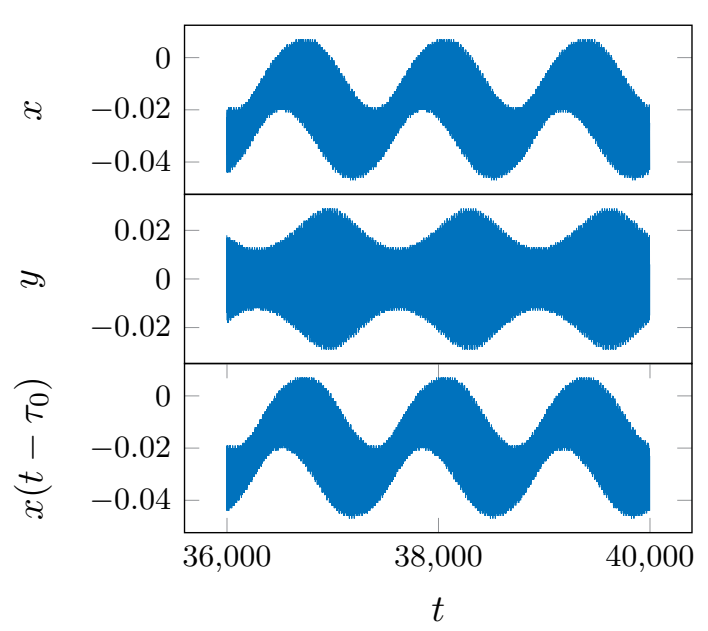

(c)

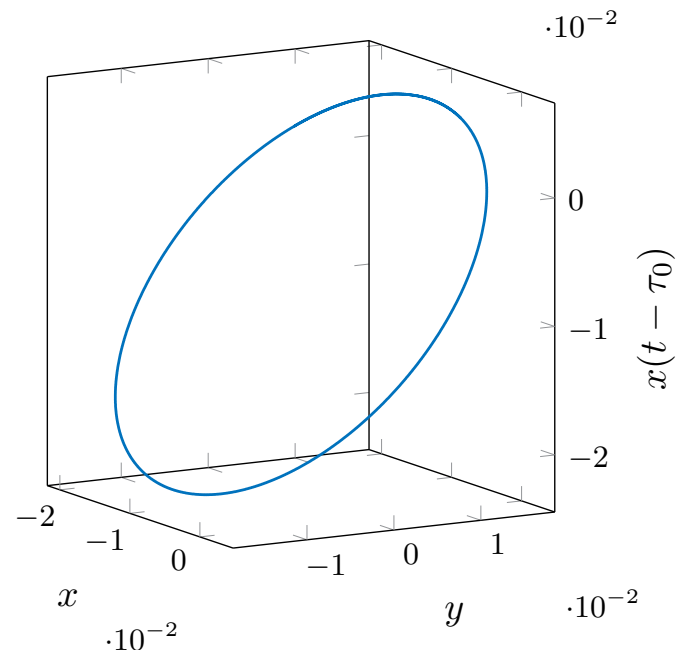

(b)

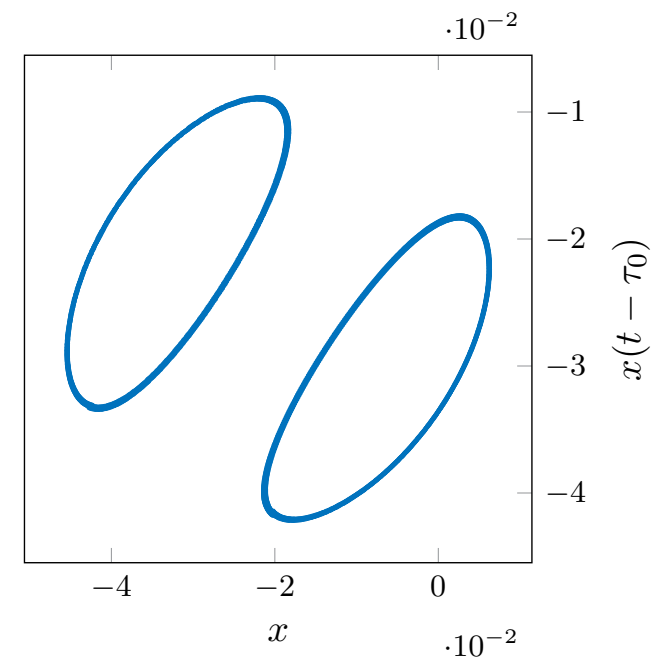

(d)

Figure S17: In (a) and (b), the periodic solution is shown to be present for parameter values in region III of Figure S15. In (c), the torus present in IV of Figure S15 is shown. In (d), a cross-section of the torus with $y(t)=-0.009$ in the phase-space $\left(x, x\left(t-\tau_{0}\right), y\right)$ is taken. 


\section{References for main text and supplement}

[1] R. Agrawal, D. Jana, R. K. Upadhyay, and V. S. H. RaO, Complex dynamics of sexually reproductive generalist predator and gestation delay in a food chain model: double Hopf-bifurcation to chaos, Journal of Applied Mathematics and Computing, (2016), pp. 1-35, doi:10.1007/s12190016-1048-1.

[2] W.-J. Beyn, A. Champneys, E. Doedel, W. Govaerts, Yu. A. Kuznetsov, and B. SandSTEDE, Numerical continuation, and computation of normal forms, in Handbook of dynamical systems, Vol. 2, North-Holland, Amsterdam, 2002, pp. 149-219, doi:10.1016/S1874-575X(02)80025-X.

[3] J. Bramburger, B. Dionne, and V. G. LeBlanc, Zero-Hopf bifurcation in the Van der Pol oscillator with delayed position and velocity feedback, Nonlinear Dynam., 78 (2014), pp. 2959-2973, doi:10.1007/s11071-014-1638-0.

[4] P. Clément, O. Diekmann, M. Gyllenberg, H. J. A. M. Heijmans, and H. R. Thieme, Perturbation theory for dual semigroups. I. the sun-reflexive case, Math. Ann., 277 (1987), pp. 709 725, doi:10.1007/BF01457866.

[5] P. Clément, O. Diekmann, M. Gyllenberg, H. J. A. M. Heijmans, and H. R. Thieme, Perturbation theory for dual semigroups. II. time-dependent perturbations in the sun-reflexive case, Proc. Roy. Soc. Edinburgh Sect. A, 109 (1988), pp. 145-172, doi:10.1017/S0308210500026731.

[6] P. Clément, O. Diekmann, M. Gyllenberg, H. J. A. M. Heijmans, and H. R. Thieme, Perturbation theory for dual semigroups. III. nonlinear Lipschitz continuous perturbations in the sun-reflexive case, in Volterra integrodifferential equations in Banach spaces and applications (Trento, 1987), vol. 190 of Pitman Res. Notes Math. Ser., Longman Sci. Tech., Harlow, 1989, pp. 67-89.

[7] P. Clément, O. Diekmann, M. Gyllenberg, H. J. A. M. Heijmans, and H. R. Thieme, Perturbation theory for dual semigroups. IV. the intertwining formula and the canonical pairing, in Semigroup Theory and Applications (Trieste, 1987), vol. 116 of Lecture Notes in Pure and Appl. Math., Dekker, New York, 1989, pp. 95-116.

[8] P. H. Coullet And E. A. Spiegel, Amplitude equations for systems with competing instabilities, SIAM J. Appl. Math., 43 (1983), pp. 776-821, doi:10.1137/0143052.

[9] V. De Witte, F. Della Rossa, W. Govaerts, and Y. A. Kuznetsov, Numerical periodic normalization for codim 2 bifurcations of limit cycles: computational formulas, numerical implementation, and examples, SIAM J. Appl. Dyn. Syst., 12 (2013), pp. 722-788, doi:10.1137/120874904.

[10] V. De Witte, W. Govaerts, Y. A. Kuznetsov, and H. G. E. Meijer, Analysis of bifurcations of limit cycles with Lyapunov exponents and numerical normal forms, Phys. D, 269 (2014), pp. 126-141, doi:10.1016/j.physd.2013.12.002.

[11] O. Diekmann, P. Getto, And M. Gyllenberg, Stability and bifurcation analysis of Volterra functional equations in the light of suns and stars, SIAM Journal on Mathematical Analysis, 39 (2007), pp. 1023-1069, doi:10.1137/060659211.

[12] O. Diekmann and M. Gyllenberg, Abstract delay equations inspired by population dynamics, in Functional Analysis and Evolution Equations, Birkhäuser, 2008, pp. 187-200, doi:10.1007/9783-7643-7794-6_12.

[13] O. Diekmann and M. Gyllenberg, Equations with infinite delay: blending the abstract and the concrete, J. Differential Equations, 252 (2012), pp. 819-851, doi:10.1016/j.jde.2011.09.038. 
[14] O. Diekmann, S. A. van Gils, S. M. Verduyn Lunel, and H.-O. Walther, Delay Equations: Functional-, Complex-, and Nonlinear Analysis, Applied Mathematical Sciences, Springer, 1995, doi:10.1007/978-1-4612-4206-2.

[15] K. Dijkstra, S. A. van Gils, S. G. Janssens, Yu. A.. Kuznetsov, and S. Visser, Pitchfork-Hopf bifurcations in 1D neural field models with transmission delays, Phys. D, 297 (2015), pp. 88-101, doi:10.1016/j.physd.2015.01.004.

[16] Y. Ding, J. CAO, AND W. JIANG, Double Hopf bifurcation in active control system with delayed feedback: application to glue dosing processes for particleboard, Nonlinear Dynam., 83 (2016), pp. 1567-1576, doi:10.1007/s11071-015-2431-4.

[17] Y. Ding And W. Jiang, Double Hopf bifurcation and chaos in Liu system with delayed feedback, J. Appl. Anal. Comput., 1 (2011), pp. 325-349, doi:10.11948/2011023.

[18] Y. Ding, W. JiAng, AND P. YU, Double Hopf bifurcation in a container crane model with delayed position feedback, Appl. Math. Comput., 219 (2013), pp. 9270-9281, doi:10.1016/j.amc.2013.03.023.

[19] K.-J. Engel And R. Nagel, One-Parameter Semigroups for Linear Evolution Equations, vol. 194 of Graduate Texts in Mathematics, Springer, New York, 2000, doi:10.1007/b97696.

[20] K.-J. Engel And R. Nagel, A Short Course on Operator Semigroups, Universitext, Springer, 2006, doi:10.1007/0-387-36619-9.

[21] K. Engelborghs, T. Luzyanina, and D. Roose, Numerical bifurcation analysis of delay differential equations using DDE-BIFTOOL, ACM Trans. Math. Software, 28 (2002), pp. 1-21, doi:10.1145/513001.513002.

[22] T. FARIA And L. T. MagalHÃes, Normal forms for retarded functional-differential equations and applications to Bogdanov-Takens singularity, J. Differential Equations, 122 (1995), pp. 201-224, doi:10.1006/jdeq.1995.1145.

[23] T. Faria and L. T. Magalhães, Normal forms for retarded functional differential equations with parameters and applications to Hopf bifurcation, Journal of differential equations, 122 (1995), pp. 181-200, doi:10.1006/jdeq.1995.1144.

[24] V. FlunkeRT AND E. SChöLL, pydelay - a python tool for solving delay differential equations, Nov. 2009, arXiv:0911.1633 [nlin.CD].

[25] J. GE AND J. XU, An efficient method for studying fold-Hopf bifurcation in delayed neural networks, Internat. J. Bifur. Chaos Appl. Sci. Engrg., 21 (2011), pp. 1393-1406, doi:10.1142/S0218127411029100.

[26] E. Geerardyn And N. Schlömer, matlab2tikz: a MATLAB/Octave script to convert native MATLAB/Octave figures to TikZ/Pgfplots figures, 2008, https://github.com/matlab2tikz/ matlab2tikz (accessed 2017/03/03). Version 1.1.0.

[27] W. Govaerts, R. K. Ghaziani, Y. A. Kuznetsov, and H. G. E. Meijer, Numerical methods for two-parameter local bifurcation analysis of maps, SIAM J. Sci. Comput., 29 (2007), pp. 26442667, doi:10.1137/060653858.

[28] W. J. F. Govaerts, Numerical Methods for Bifurcations of Dynamical Equilibria, Society for Industrial and Applied Mathematics, Philadelphia, PA, 2000, doi:10.1137/1.9780898719543. 
[29] S. Guo, Y. Chen, And J. Wu, Two-parameter bifurcations in a network of two neurons with multiple delays, Journal of differential equations, 244 (2008), pp. 444-486, doi:10.1016/j.jde.2007.09.008.

[30] S. Guo And J. Man, Center manifolds theorem for parameterized delay differential equations with applications to zero singularities, Nonlinear Anal., 74 (2011), pp. 4418-4432, doi:10.1016/j.na.2011.04.003.

[31] J. K. HALE, Theory of Functional Differential Equations, Springer, second ed., 1977, doi:10.1007/978-1-4612-9892-2. Applied Mathematical Sciences, Vol. 3.

[32] J. K. Hale and S. M. Verduyn Lunel, Introduction to Functional Differential Equations, vol. 99 of Applied Mathematical Sciences, Springer, 1993, doi:10.1007/978-1-4612-4342-7.

[33] J. Hindmarsh and R. Rose, A model of the nerve impulse using two first-order differential equations, Nature, 296 (1982), pp. 162-164, doi:10.1038/296162a0.

[34] J. L. Hindmarsh And R. M. Rose, A model of neuronal bursting using three coupled first order differential equations, Proceedings of the Royal Society of London B: Biological Sciences, 221 (1984), pp. 87-102, doi:10.1098/rspb.1984.0024.

[35] A.-V. Ion, An example of Bautin-type bifurcation in a delay differential equation, J. Math. Anal. Appl., 329 (2007), pp. 777-789, doi:10.1016/j.jmaa.2006.06.083.

[36] A.-V. Ion And R. M. Georgescu, Bautin bifurcation in a delay differential equation modeling leukemia, Nonlinear Anal., 82 (2013), pp. 142-157, doi:10.1016/j.na.2013.01.009.

[37] S. G. Janssens, On a Normalization Technique for Codimension Two Bifurcations of Equilibria of Delay Differential Equations, master's thesis, Utrecht University, The Netherlands, 2010, https://dspace.library.uu.nl/handle/1874/312252. Corrections and updates are available via http://sebastiaanjanssens.nl/pdf/normalization.pdf.

[38] S. G. Janssens, A class of abstract delay differential equations in the light of suns and stars, Jan. 2019, arXiv:1901.11526 [math.DS].

[39] H. Jiang, T. Zhang, And Y. Song, Delay-induced double Hopf bifurcations in a system of two delay-coupled van der Pol-Duffing oscillators, Internat. J. Bifur. Chaos Appl. Sci. Engrg., 25 (2015), pp. 1550058, 18, doi:10.1142/S0218127415500583.

[40] W. Jiang And H. Wang, Hopf-transcritical bifurcation in retarded functional differential equations, Nonlinear Anal., 73 (2010), pp. 3626-3640, doi:10.1016/j.na.2010.07.043.

[41] H. B. KeLler, Lectures on numerical methods in bifurcation problems, vol. 79 of Tata Institute of Fundamental Research Lectures on Mathematics and Physics, Published for the Tata Institute of Fundamental Research, Bombay; by Springer-Verlag, Berlin, 1987. With notes by A. K. Nandakumaran and Mythily Ramaswamy.

[42] Yu. A. Kuznetsov, Numerical normalization techniques for all codim 2 bifurcations of equilibria in ODEs, SIAM Journal on Numerical Analysis, 36 (1999), pp. 1104-1124, doi:10.1137/S0036142998335005.

[43] Yu. A. Kuznetsov, Elements of Applied Bifurcation Theory, vol. 112 of Applied Mathematical Sciences, Springer, third ed., 2004, doi:10.1007/978-1-4757-3978-7.

[44] Yu. A. Kuznetsov, W. Govaerts, E. J. Doedel, and A. Dhooge, Numerical periodic normalization for codim 1 bifurcations of limit cycles, SIAM J. Numer. Anal., 43 (2005), pp. 14071435, doi:10.1137/040611306. 
[45] Yu. A. Kuznetsov, H. G. E. Meijer, B. Al Hdaibat, and W. Govaerts, Improved homoclinic predictor for Bogdanov-Takens bifurcation, Internat. J. Bifur. Chaos Appl. Sci. Engrg., 24 (2014), pp. 1450057, 12, doi:10.1142/S0218127414500576.

[46] Yu. A. Kuznetsov, H. G. E. Meijer, W. Govaerts, and B. Sautois, Switching to nonhyperbolic cycles from codim 2 bifurcations of equilibria in ODEs, Phys. D, 237 (2008), pp. 3061-3068, doi:10.1016/j.physd.2008.06.006.

[47] M. Lichtner, Variation of constants formula for hyperbolic systems, J. Appl. Anal., 15 (2009), pp. 79-100, doi:10.1515/JAA.2009.79.

[48] S. Ma And Z. Feng, Fold-Hopf bifurcations of the Rose-Hindmarsh model with time delay, Internat. J. Bifur. Chaos Appl. Sci. Engrg., 21 (2011), pp. 437-452, doi:10.1142/S0218127411028490.

[49] H. G. E. Meijer, F. Dercole, and B. Oldeman, Numerical bifurcation analysis, in Mathematics of Complexity and Dynamical Systems. Vols. 1-3, Springer, 2012, pp. 1172-1194, doi:10.1007/978-1-4614-1806-1_71.

[50] J. Peng, L. WAng, Y. ZhaO, AND Y. ZhaO, Bifurcation analysis in active control system with time delay feedback, Appl. Math. Comput., 219 (2013), pp. 10073-10081, doi:10.1016/j.amc.2013.04.014.

[51] R. Qesmi And M. A. Babram, Double Hopf bifurcation in delay differential equations, Arab Journal of Mathematical Sciences, 20 (2014), pp. 280 - 301, doi:10.1016/j.ajmsc.2013.10.002.

[52] A. F. Ruston, Fredholm Theory in Banach Spaces, vol. 86 of Cambridge Tracts in Mathematics, Cambridge University Press, Cambridge, 1986, doi:10.1017/CBO9780511569180.

[53] L. F. Shampine and S. Thompson, Solving delay differential equations with dde23, Appl. Numer. Math, (2001), pp. 441-458.

[54] Z. Shen and C. Zhang, Double Hopf bifurcation of coupled dissipative Stuart-Landau oscillators with delay, Appl. Math. Comput., 227 (2014), pp. 553-566, doi:10.1016/j.amc.2013.11.044.

[55] J. Sieber, Local bifurcations in differential equations with state-dependent delay, Chaos: An Interdisciplinary Journal of Nonlinear Science, 27 (2017), p. 114326, doi:10.1063/1.5011747, arXiv:1705.07550 [math.DS].

[56] J. Sieber, K. Engelborghs, T. Luzyanina, G. Samaey, and D. Roose, DDE-BIFTOOL Manual - Bifurcation analysis of delay differential equations, June 2014, arXiv:1406.7144 [math.DS].

[57] Z. Song AND J. Xu, Bursting near Bautin bifurcation in a neural network with delay coupling, International Journal of Neural Systems, 19 (2009), pp. 359-373, doi:10.1142/S0129065709002087. PMID: 19885964.

[58] A. E. TaYlor And D. C. Lay, Introduction to Functional Analysis, John Wiley \& Sons, New York, second ed., 1980.

[59] S. A. van Gils, S. G. Janssens, Yu. A. Kuznetsov, and S. Visser, On local bifurcations in neural field models with transmission delays, Journal of Mathematical Biology, 66 (2013), pp. 837887, doi:10.1007/s00285-012-0598-6, arXiv:1209.2849 [math.DS].

[60] B. WAGE, Normal form computations for delay differential equations in DDE-BIFTOOL, master's thesis, Utrecht University, The Netherlands, 2014, https://dspace.library.uu.nl/handle/ $1874 / 296912$. 
[61] Y. WANG, H. WANG, AND W. JiAng, Hopf-transcritical bifurcation in toxic phytoplanktonzooplankton model with delay, J. Math. Anal. Appl., 415 (2014), pp. 574-594, doi:10.1016/j.jmaa.2014.01.081.

[62] X. P. Wu, Zero-Hopf bifurcation analysis of a Kaldor-Kalecki model of business cycle with delay, Nonlinear Anal. Real World Appl., 13 (2012), pp. 736-754, doi:10.1016/j.nonrwa.2011.08.013.

[63] J. Xu And K. W. Chung, Effects of time delayed position feedback on a van der Pol-Duffing oscillator, Phys. D, 180 (2003), pp. 17-39, doi:10.1016/S0167-2789(03)00049-6.

[64] Y. Xu AND T. Shi, Computation of double Hopf points for delay differential equations, Open Math., 13 (2015), pp. 805-815, doi:10.1515/math-2015-0076.

[65] B. Zhen AND J. XU, Bautin bifurcation analysis for synchronous solution of a coupled FHN neural system with delay, Commun. Nonlinear Sci. Numer. Simul., 15 (2010), pp. 442-458, doi:10.1016/j.cnsns.2009.04.006.

[66] B. Zhen And J. XU, Fold-Hopf bifurcation analysis for a coupled FitzHugh-Nagumo neural system with time delay, Internat. J. Bifur. Chaos Appl. Sci. Engrg., 20 (2010), pp. 3919-3934, doi:10.1142/S0218127410028112. 